\title{
SUMOylation modulates $\alpha$-synuclein toxicity and fibril formation
}

\author{
PhD Thesis \\ in partial fulfilment of the requirements \\ for the degree "Doctor of Philosophy (PhD)" \\ in the Molecular Biology Program \\ at the Georg August University Göttingen, \\ Faculty of Biology
}

submitted by

Petranka Krumova

born in

Sofia, Bulgaria

March 2009 


\section{Declaration}

I hereby declare that the thesis: "SUMOylation modulates a-synuclein toxicity and fibril formation" has been written independently and with no other sources and aids than quoted.

Petranka Krumova

Göttingen, March 2009 


\title{
Acknowledgements
}

\author{
I would like to thank all the people who supported me during the work on this PhD \\ thesis, especially \\ Dr. Jochen Weishaupt for his supervision, continuous support and for the opportunity to work \\ independently in his group;
}

Prof. Mathias Bähr for giving me the chance to work in his laboratory and his guidance during my doctoral work;

Prof. Frauke Melchior for her great support, generosity and involvement in my PhD project. Many thanks for the advice and the motivation in the difficult moments during those years. Thanks to the members of the Melchior's lab for sharing reagents end equipment with me;

Dr. Erik Meulmeester for all his help and the fruitful discussions we had. I benefited a lot from our collaboration and it was a great pleasure for me to work with him;

Prof. Klaus-Armin Nave for being part of my PhD Thesis Committee and the feed-back on the project during the years of PhD;

NEUREST for financing my fellowship and the opportunity to participate in numerous conferences and professional courses;

Our collaborators - He-Hsuan Hsiao and Dr. Henning Urlaub who performed the mass spectrometry experiments; Dr. Marilyn Tirard and Prof. Nils Brose for providing the SUMO transgenic mice; Pinar Karpinar and Dr. Markus Zweckstetter for their help with the aggregation assay; Dr. Manuel Garrido and Dr. Sebastian Kügler for their assistance in the in vivo experiments; Dr. Mika Ruonala for the FRET/FLIM quantification;

Current and former members of the lab, in particular Dr. Katrin Eckermann, Chrisitne Poser, Dr. Katrin Meuer and Dr. Elena Kvashnina for the nice working atmosphere; Christine Poser for her excellent technical support and for correcting the Methods section of this thesis, Katrin Eckermann for exciting discussions, the critical reading and the valuable comments on my thesis; special thanks to Katrin Meuer and Christine Poser for supporting my attempts in learning German;

Manuel Garrido for his help with the animal injections, his advice and especially for the nice discussions;

My dear friends, Evelina and Blago, always being there for me to share happy and sad moments, for their understanding and the great weekends we spent together;

My dear Miś, Lukasz, for his love and support, for being next to me and helping me in so many things, which I'm not able to list here;

Last but not least, my family for forgiving me the long distance and time spent away from home, for their continuous love, support and encouragement in every moment of my life. 


\section{Table of Contents}

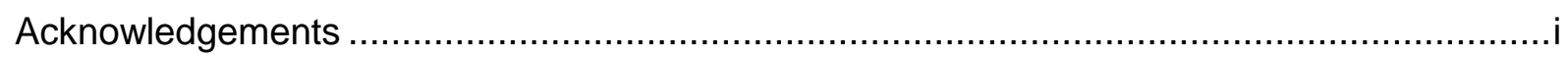

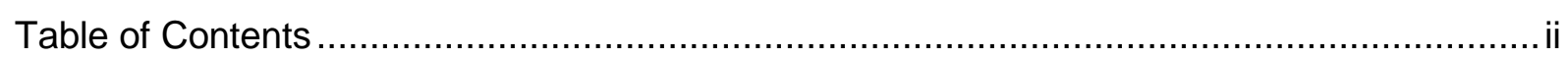

List of Figures and Tables ........................................................................................

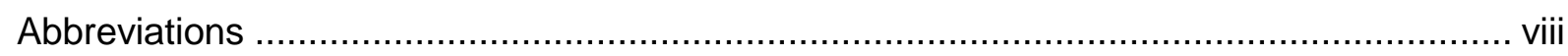

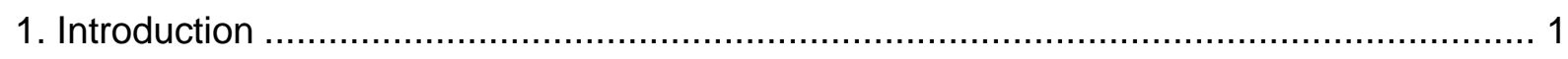

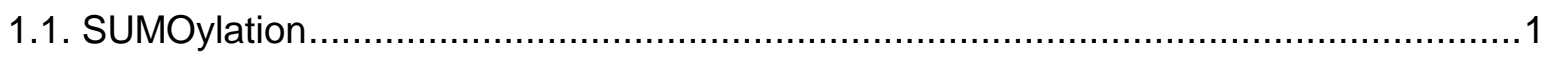

1.1.1. The family of Small Ubiquitin-like Modifiers.....................................................

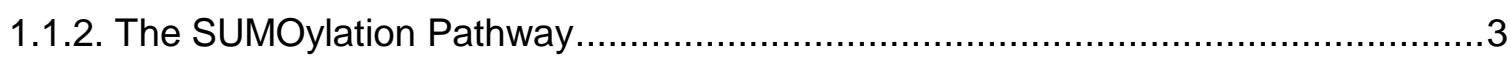

1.1.3. Molecular and functional effects of SUMOylation ...........................................

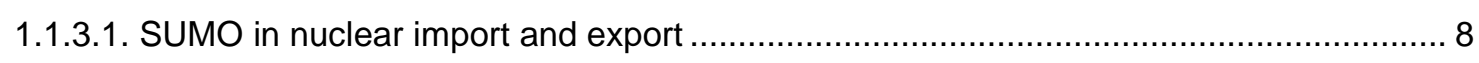

1.1.3.2. SUMOylation interferes with protein activity ......................................................... 8

1.1.3.3. SUMOylation induces conformational changes..................................................... 9

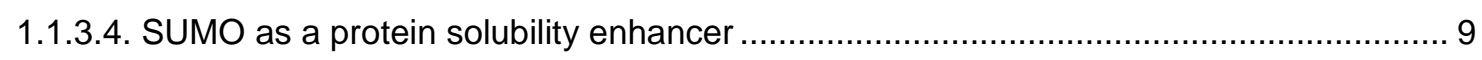

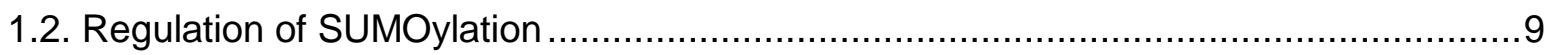

1.3. SUMOylation as a posttranslational modification in the CNS ..................................11

1.4. SUMO in neurodegenerative disorders ........................................................ 12

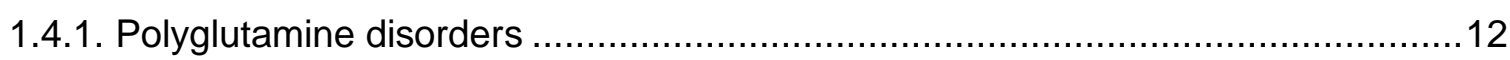

1.4.2. Neuronal intranuclear inclusion disease (NIID) ........................................ 13

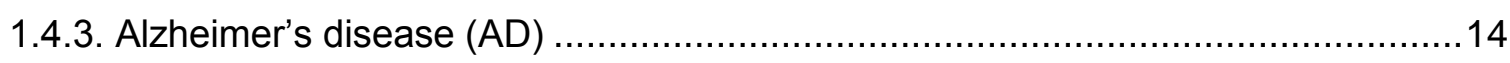

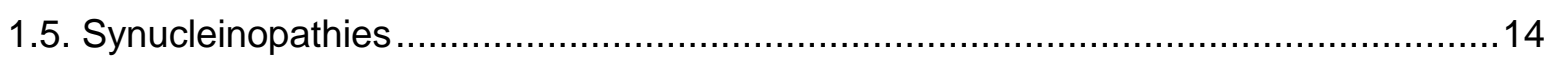

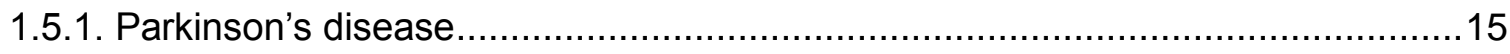

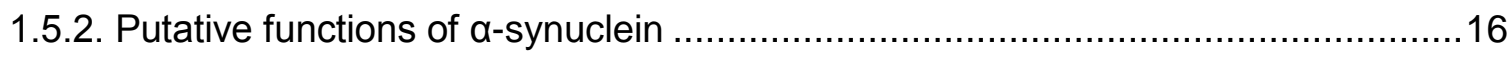

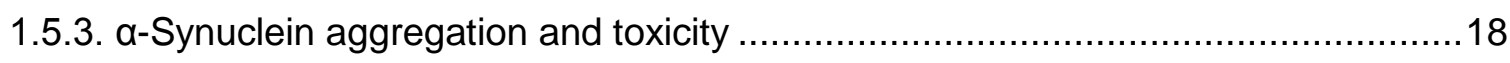

1.5.4. $\alpha$-Synuclein is subject to various posttranslational modifications .........................19

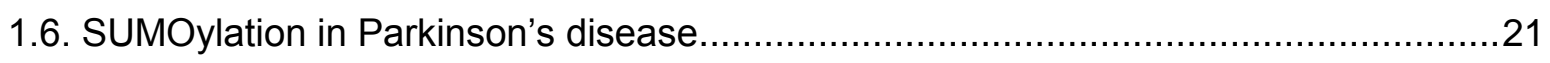

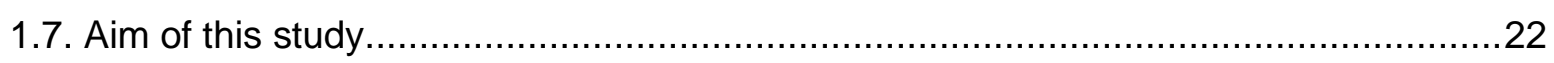

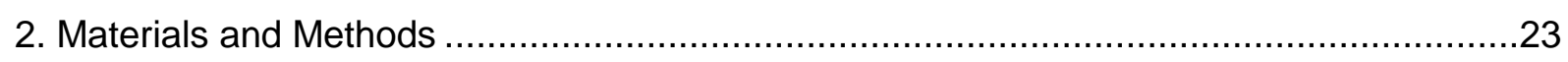

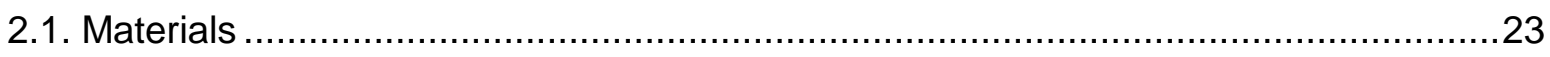

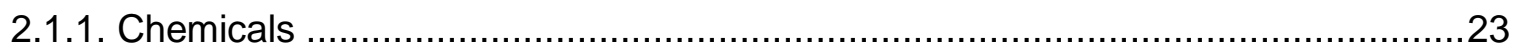

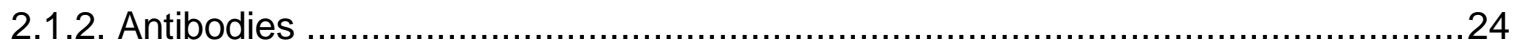

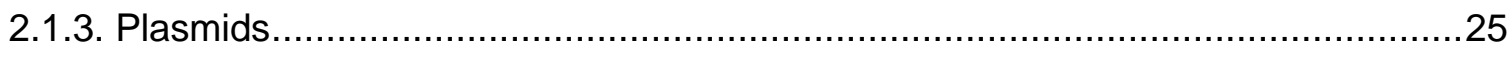

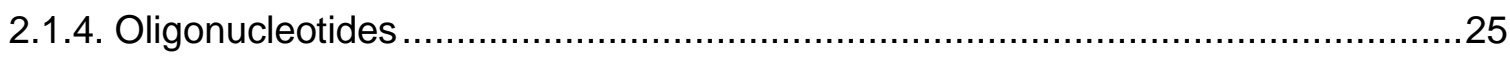

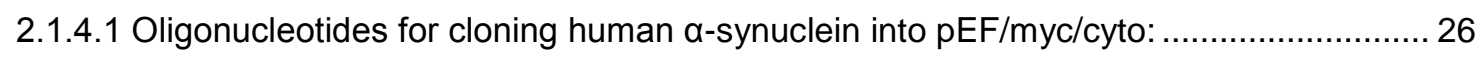

2.1.4.2. Oligonucleotides for cloning $\alpha$-synuclein mutants via site-directed PCR mutagenesis: 
2.1.4.3. Oligonucleotides for cloning $\alpha$-synuclein-KO and $\alpha$-synuclein-DM mutants into pT7-7 bacterial expression vector:

2.1.4.4. Oligonucleotides for cloning $\alpha$-synuclein-KO10 and $\alpha$-synuclein-DM mutants into rAAV-

2 vector:

2.1.4.5. Oligonucleotides for mVenus cDNA amplification and cloning of $m V e n u s-S U M O \triangle 4$ and

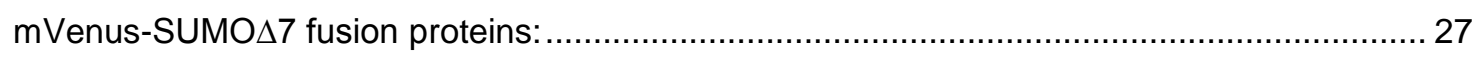

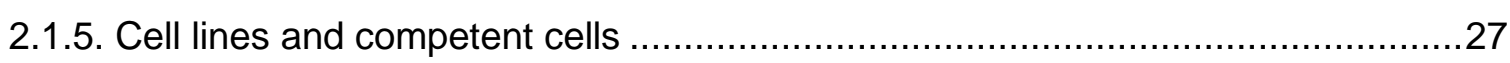

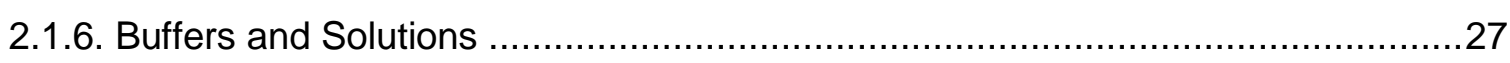

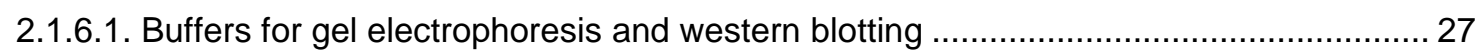

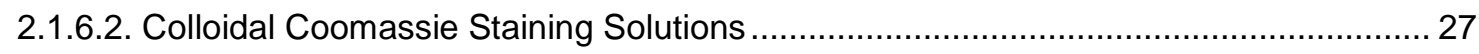

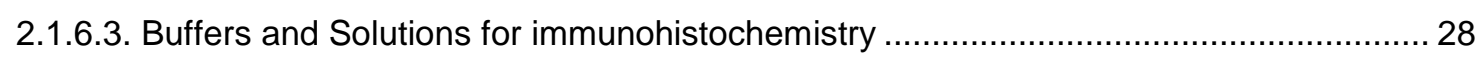

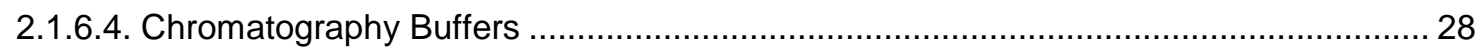

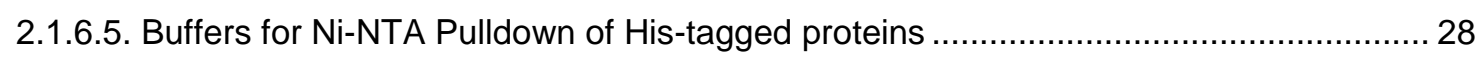

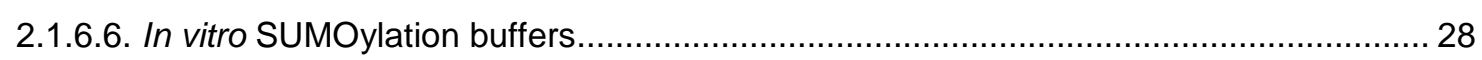

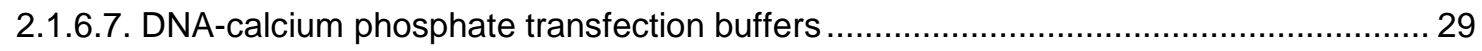

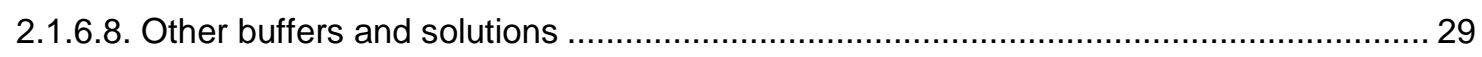

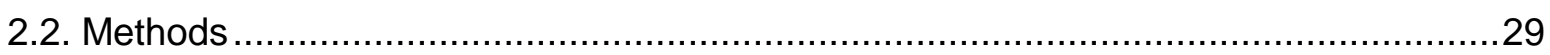

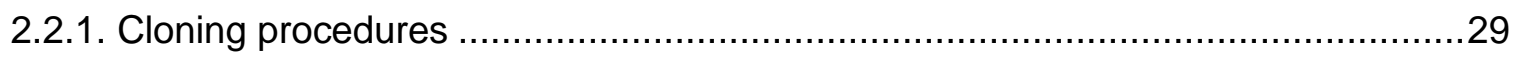

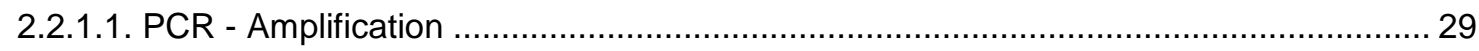

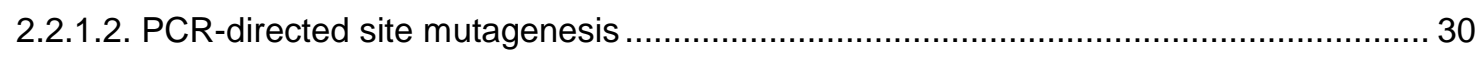

2.2.1.3. DNA restriction, electrophoresis, gel extraction, concentration determination.............. 31

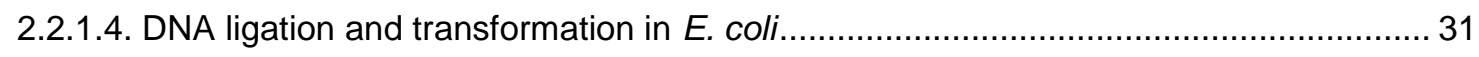

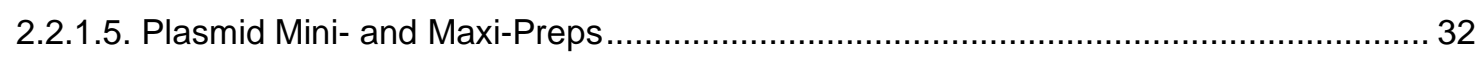

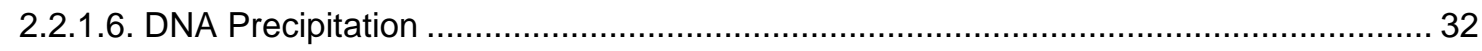

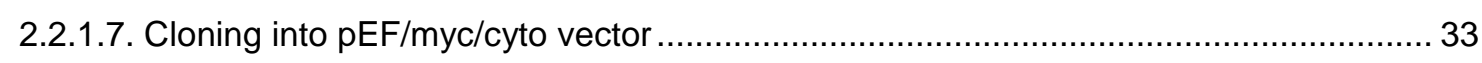

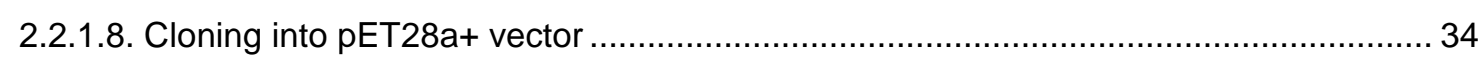

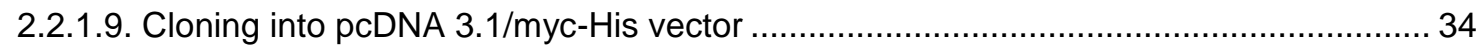

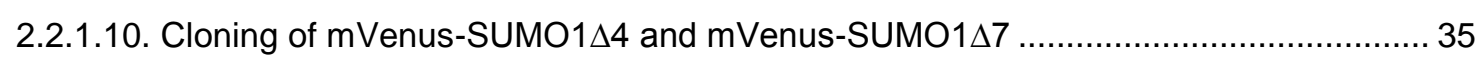

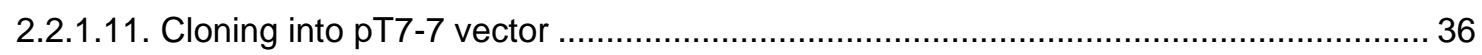

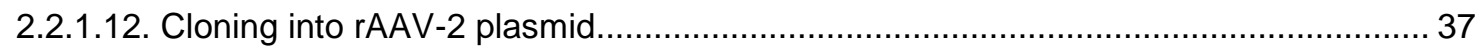

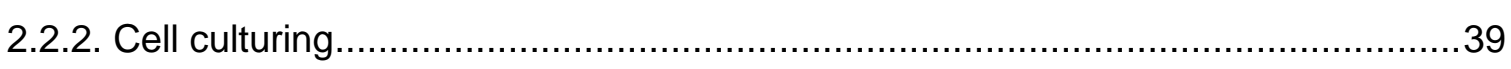

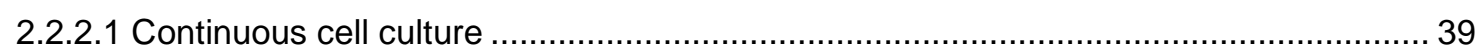

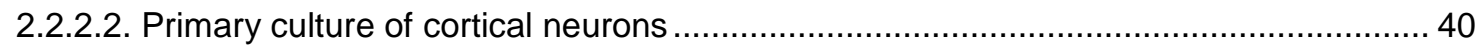

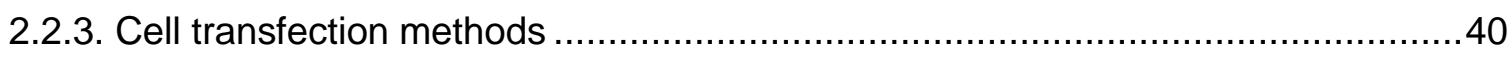

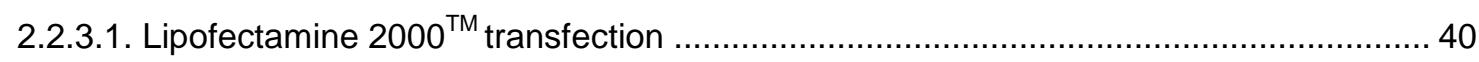

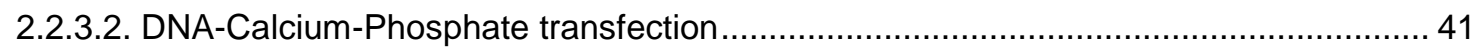

2.2.4. Viral vector production and purification ....................................................... 41

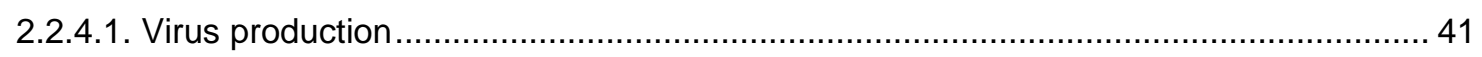

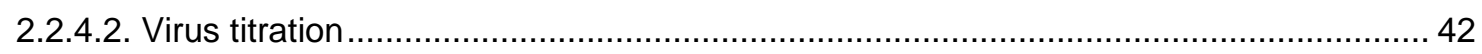

2.2.5. Identification of SUMOylated targets................................................................ 42 
2.2.5.1. In vitro SUMOylation using recombinantly expressed proteins ................................. 42

2.2.5.2. Identification of SUMOylated proteins using Ni-NTA precipitation ................................ 42

2.2.5.3 Identification of in vivo SUMOylated proteins from brain lysates of $\mathrm{His}_{6}$-SUMO2

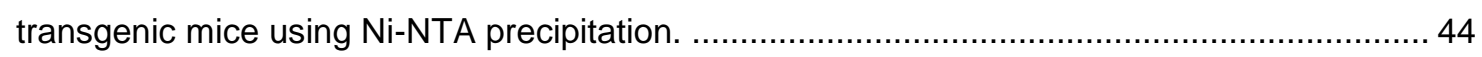

2.2.6. Identification of SUMO conjugation sites by mass spectrometry ........................44

2.2.7. Recombinant expression and purification of proteins ......................................... 45

2.2.7.1. Recombinant expression and purification of $\alpha$-synuclein ............................................... 45

2.2.7.2. Expression and purification of E.coli SUMO-modified $\alpha$-synuclein ............................. 46

2.2.8. Protein handling procedures ................................................................... 47

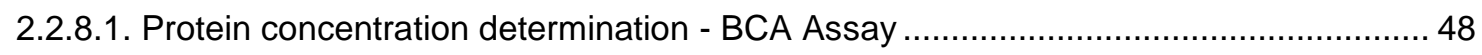

2.2.8.2. SDS-polyacrylamide gel electrophoresis (SDS-PAGE) ........................................ 48

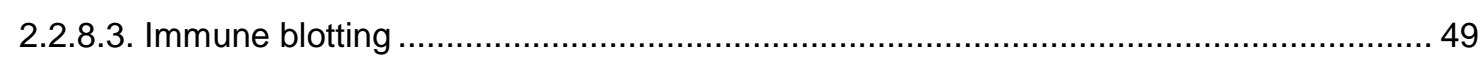

2.2.8.4. Methanol-Chloroform protein precipitation …........................................................ 50

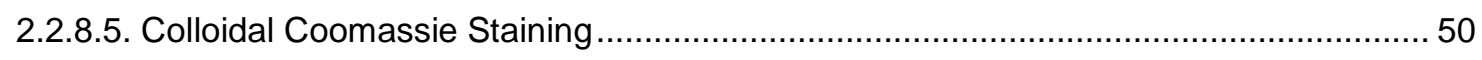

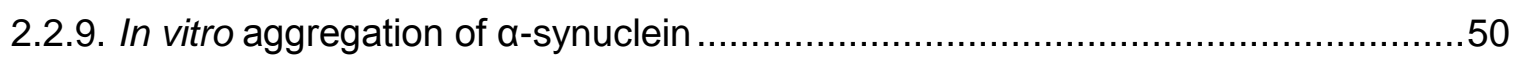

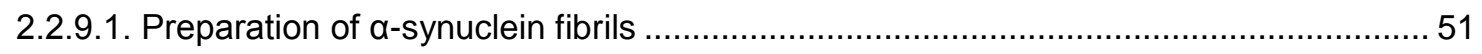

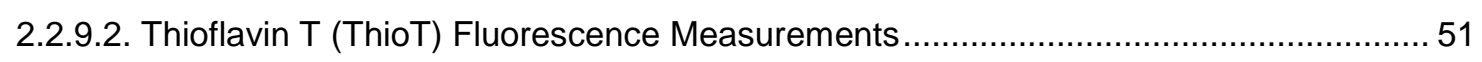

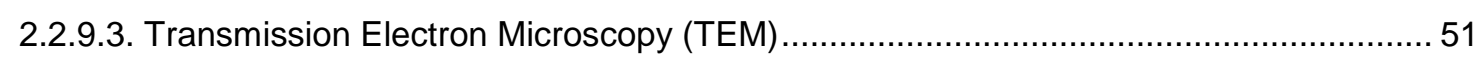

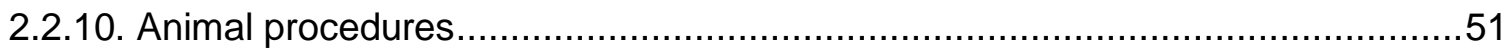

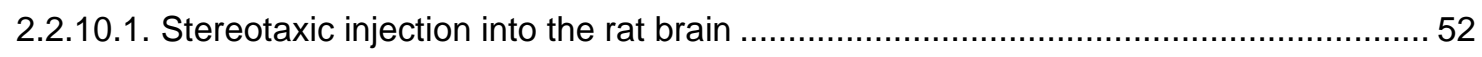

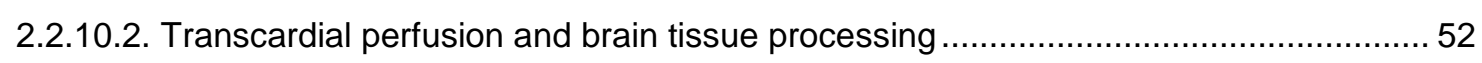

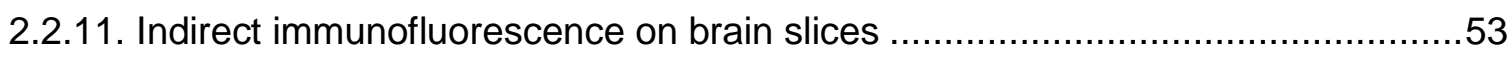

2.2.12. Quantification of VMAT-positive neurons in SNpc.......................................54

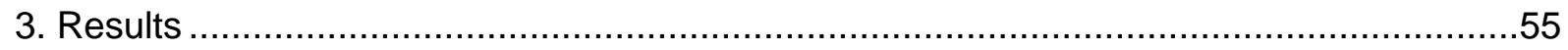

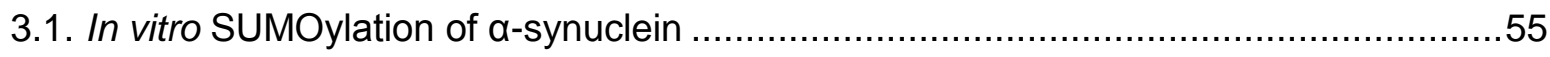

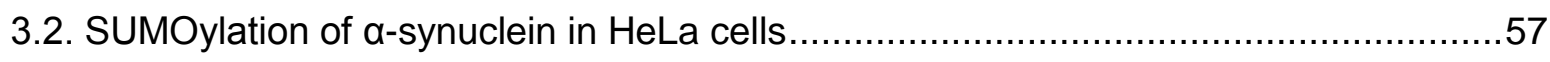

3.3. Effects of familial PD mutations on $\alpha$-synuclein SUMOylation .................................58

3.4. FRET-FLIM analysis of $\alpha$-synuclein SUMOylation in the CSM14.1 cell line ................59

3.5. $a$-Synuclein is SUMOylated in brain tissue of $\mathrm{His}_{6}$-SUMO2 transgenic mice .............62

3.6. Identification of $\alpha$-synuclein SUMOylation sites ..............................................65

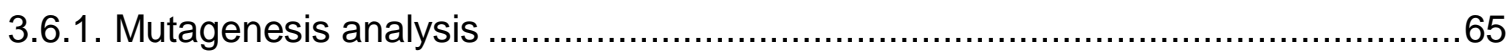

3.6.2. Overproduction of SUMOylated $\alpha$-synuclein in E.coli and mass spectrometry .....69

3.7. Ubiquitination of $\alpha$-synuclein WT, DM and KO10 in HEK 293 cells.........................74

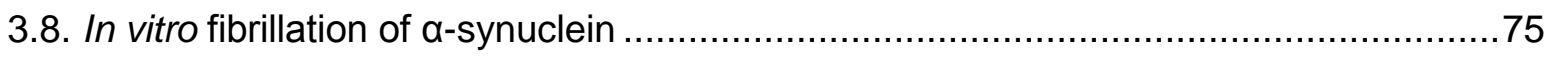

3.8.1 Overexpression and purification of non-tagged SUMOylated $\alpha$ - synuclein ............76

3.8.2. SUMOylation inhibits $\alpha$-synuclein fibrillation in vitro ........................................ 78

3.9. The role of $\alpha$-synuclein SUMOylation in dopaminergic cell survival ...........................82

3.10. $\alpha$-Synuclein-DM has a higher aggregation rate and is more toxic in HEK 293 cells. .88

4. Discussion 


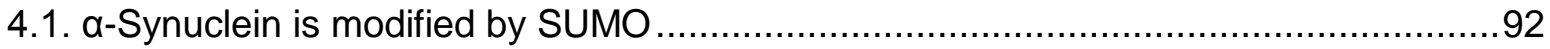

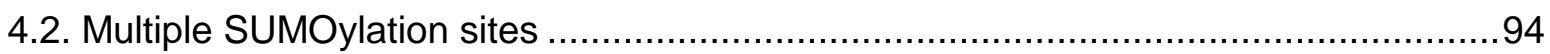

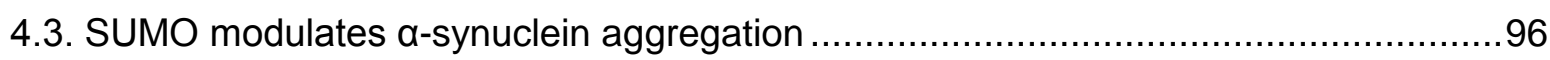

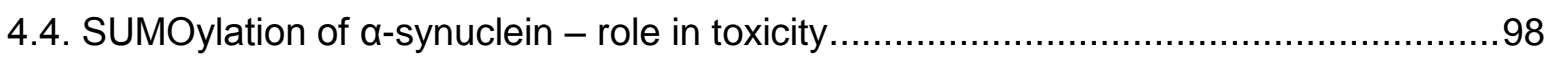

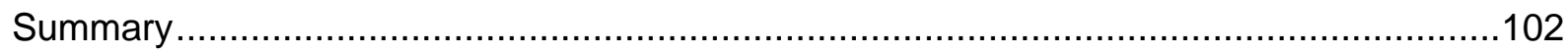

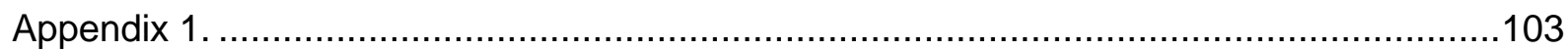

The Cyclin-dependent kinase 5 regulator p35 is a novel SUMOylation target ................103

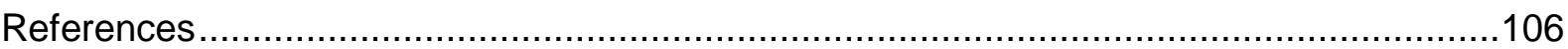

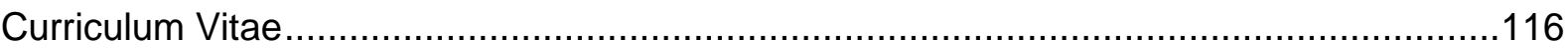

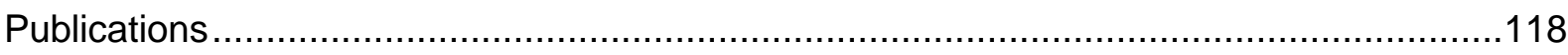




\section{List of Figures and Tables}

Figure 1-1. Representation of the 3D NMR structures of ubiquitin, SUMO1 and SUMO2/3... 2

Figure 1-2. The SUMO cycle. 4

Figure 1-3. Effects of SUMOylation on molecular level. 7

Table 1. Genes and loci linked to familial PD. 15

Figure 1-4. Schematic depiction of $\alpha$-synuclein amino acid sequence. 17

Figure 1-5. $\alpha$-Synuclein misfolding and aggregation and the downstream consequences. ...18

Figure 2-1. Schematic depiction of $\alpha$-synuclein-WT subcloned into $\mathrm{pEF/myc/cyto} \mathrm{vector.} \mathrm{....34}$

Figure 2-2. Schematic depiction of $\alpha$-synuclein-WT cloned into pcDNA3.1/myc/His. .35

Figure 2-3. Schematic depiction of mVenus-SUMO $\Delta 4 / \Delta 7$ in $\mathrm{pEYFP-C1}$ vector backbone....36

Figure 2-4. Schematic depiction of $\alpha$-synuclein-WT cloned into pT7-7 expression vector; ....36

Figure 2-5. Schematic depiction of pAAV2-synuclein. .38

Figure 2-6. Schematic depiction of $\alpha$-synuclein in pMA vector. 39

Figure 2-7. Schematic representation of the E.coli SUMO-modification system..... .46

Figure 3-1. In vitro SUMOylation of $\alpha$-synuclein..... .56

Figure 3-2. In vitro conjugation of SUMO2 to $\alpha$-synuclein .57

Figure 3-3. a-Synuclein is modified by SUMO in HeLa cells. .58

Figure 3-4. Familial Parkinson's disease related mutants A30P and A53T are SUMOylated.

Figure 3-5. SUMOylation of $\alpha$-synuclein detected by FRET/FLIM in intact single cells. .61

Figure 3-6. Generation of Thy1.2: His6-SUMO2 transgenic mice .63

Figure 3-7. In vivo SUMOylation of $\alpha$-synuclein .64

Figure 3-8. Mutagenesis analysis of $\alpha$-synuclein in HEK 293 cells. 66

Figure 3-9. a-Synuclein-DM and $\alpha$-synuclein-KO show reduction in SUMOylation in HEK 293 cells.

Figure 3-10. a-Synuclein-DM and a-synuclein-KO show a reduction in SUMOylation in HeLa cells. 68

Figure 3-11. Confirmation of $\alpha$-synuclein consensus SUMOylation sites by mutations of Asp/Glu to Ala. 
Figure 3-12. Separation of SUMO modified and non-modified $\alpha$-synuclein.

Figure 3-13. Summary of $\alpha$-synuclein SUMOylation sites identified by mass spectrometry. 72

Figure 3-14. Mass spectrometry reveals SUMO conjugation at K96 and K102. .73

Figure 3-15. Ubiquitination of $\alpha$-synuclein-WT, DM and KO10. .75

Figure 3-16. Purification of non-tagged SUMOylated $\alpha$-synuclein. .77

Figure 3-17. Coomassie staining of recombinantly purified proteins used in the in vitro fibrillization assay.

Figure 3-18. In vitro fibrillization of $\alpha$-synuclein. .80

Figure 3-19. Transmission electron microscopy (TEM) of in vitro aggregation samples .81

Figure 3-20. Schematic representation of vector genomes used in the in vivo rat model of PD.

Figure 3-21. Cultured primary rat cortical neurons transduced with AAV2 vectors encoding $\alpha$ synuclein WT, DM and KO10. . .83

Figure 3-22. Unilateral rAAV-mediated transduction of SNpc. 85

Figure 3-23. Photomicrographs showing nigral degeneration in rAAV- $\alpha$-synuclein-injected animals.

Figure 3-24. Unbiased estimation of VMAT2+ cells in the SNpc of animals injected with wild type or mutant human a-synuclein.

Figure 3-25. Comparison of GFP distribution patterns between $\alpha$-synuclein wild type or DM using the PDZ assay. .90

Figure 4-1. Model of the role of $\alpha$-synuclein SUMOylation in neuronal cell death. 100 


\section{Abbreviations}

AAV - adeno-associated virus

AD - Alzheimer's disease

Ad - autosomal dominant

ALDH2 - Aldehyde dehydrogenase 2

Amp - Ampicillin

APP - Amyloid precursor protein

$\mathbf{A R}$ - Androgen receptor

$\mathrm{Ar}$ - autosomal recessive

$A B$ - Amyloid beta peptide

ATG - Autophagic ubiquitin-like protein

ATP - adenosine triphosphate

BCA - bicinchoninic acid

BGH - bovine growth hormone derived polyadenylation site

bp - base pairs

BSA - bovine serum albumine

cDNA - complamentary DNA

CK - Casien kinase

CNS - Central Nervous System

CREB - CAMP response element-binding protein

CSP - Cystein-string protein

DAPI - 4',6-diamidino-2-phenylindole

DLB - Dementia with Lewy bodies

DMEM - Dulbecco's modified Eagle's medium

DNA - desoxyribonucleic acid

dNTP - deoxynucleotide triphosphate

DTT - dithiothreitol

EDTA - ethylenediaminetetraacetic acid

eGFP - enhanced green fluorescent protein

ER - Endoplasmic reticulum

FAK - Focal adhesion kinase

FCS - fetal calf serum

FLIM - Fluorescence lifetime imaging

FPLC - fast protein liquid chromatography

FRET - Förster resonance energy transfer

fw - forward 
GTP - guanosine triphosphate

HD - Huntington's disease

HDAC - Histone deacetylase

HEK - human embryonic kidney

HRP - horseradish peroxidase

HSF - Heat shock transcription factor

Hsp - Heat shock protein

hSyn - human Synapsin

Htt - Huntingtin

IHC- immunohisochemistry

IPTG - isopropyl-beta-D-thiogalactopyranoside

IR1+M - Internal repeat 1 + middle domain

ISG - Interferon inducible gene

kb - kilobase

kDa - kilodalton

LB - Luria Broth

LRRK - Leucine-rich repeat kinase

MPTP - 1-methyl-4-phenyl-1,2,3,6-tetrahydropyridine

mRNA - messenger RNA

MSA - Multiple system atrophy

MWCO - molecular weight cut off

NAC - Non-Aß component of Alzheimer's disease amyloid

NBM - neurobasal medium

NEDD - Neuronal precursor cell expressed developmentally down-regulated

NEM - N-Ethylmaleimide

$\mathrm{Ni}^{2+}$-NTA - nickel nitrilotriacetic acid

NIID - Neuronal intranuclear inclusion disease

NLS - Nuclear localization signal

NMR - Nuclear magnetic resonance

NPC - Nuclear pore complex

PAGE - Polyacrylamide gel electrophoresis

PBS - phosphate buffered saline

Pc2 - Polycomb protein 2

PCNA - Proliferating cell nuclear antigen

PCR - polymerase chain reaction

PD - Parkinson's disease

PDSM - Phosphorylation dependent SUMOylation motif 
PFA - paraformaldehyde

PIAS - Protein inhibitors of activated STAT

PINK - PTEN induced putative kinase

PML - Promyelocytic leukemia protein

RanGAP - Ran GTP-ase activating protein

rev - reverse

ROS - Reactive oxygen species

rpm - rounds per minute

RSUME - RING finger and WD-repeat-containing SUMOylation enhancer

RT - room temperature

SAB - SUMOylation assay buffer

SAE - SUMO activating enzyme

SBMA - Spinobulbar muscular atrophy

SCA - Spinocerebellar ataxia

SCNA - a-synuclein gene

SDS - sodium dodecylsulfate

SENP - Sentrin specific protease

SIAH - Seven in Absentia Homolog

SIM - SUMO Interacting Motif

SNARE - Soluble NSF Attachment protein Receptors

SNpc - Substantia nigra pars compacta

SNpr - Substantia nigra pars reticulata

SUMO - Small ubiquitin-like modifier

TB - Transport buffer

TdG - Thymine DNA glycosylase

TEM - Transmission electron microscopy

ThioT - Thioflavin T

TP - total protein

TR - terminal repeats

Ubls - Ubiquitin-like modifiers

UCHL - ubiquitin carboxy-terminal hydrolase $\mathrm{L}$

VMAT - Vesicular monoamine transporter

WB - Western blot

WPRE - woodchuck hepatitis virus posttranscriptional regulatory element

WT - wild type 


\section{Introduction}

\subsection{SUMOylation}

\subsubsection{The family of Small Ubiquitin-like Modifiers}

Ubiquitin and the members of the Ubiquitin-like proteins get conjugated to proteins, altering their properties and thus hugely increasing the eukaryotic proteome complexity at a posttranslational level. Since the discovery of Ubiquitin more than 25 years ago, a dozen Ubiquitin-like proteins were discovered, among those NEDD8 (neural precursor cell expressed, developmentally down-regulated 8), ISG 15 (interferon inducible gene 15), ATG 8 (Autophagic Ubiquitin-like protein), ATG 12 and SUMO (Small Ubiquitin-like Modifier).

SUMO was first identified in mammals where it was found to be covalently conjugated to the GTPase activating protein RanGAP1 (Matunis et al., 1996; Mahajan et al., 1997).

The SUMO family members are ubiquitously expressed in all types of eukaryotic cells. Yeasts have only one SUMO gene, encoding the Smt3 protein (yeast homolog of SUMO1) (Lapenta et al., 1997), whereas the human genome encodes four distinct SUMO isoforms, named SUMO1 to SUMO4 (Melchior, 2000; Bohren et al., 2004; Guo et al., 2004). SUMO1 is about $11 \mathrm{kDa}, 101$-amino-acid protein that shares only $\approx 18 \%$ sequence homology with Ubiquitin. SUMO2 and SUMO3 are almost identical, differing among each other in only 3 amino-terminal residues, but having only $\approx 47 \%$ homology with SUMO1. SUMO1-3 are ubiquitously expressed, while SUMO4 mRNA expression is confined primarily to the lymph node, kidney and spleen (Guo et al., 2004). It is still not clear whether the SUMO4 gene product can be processed and conjugated to targets.

Despite very little sequence homology, all SUMO isoforms resemble the threedimensional structure of Ubiquitin (Bayer et al., 1998), (Figure 1-1). 
Ubiquitin

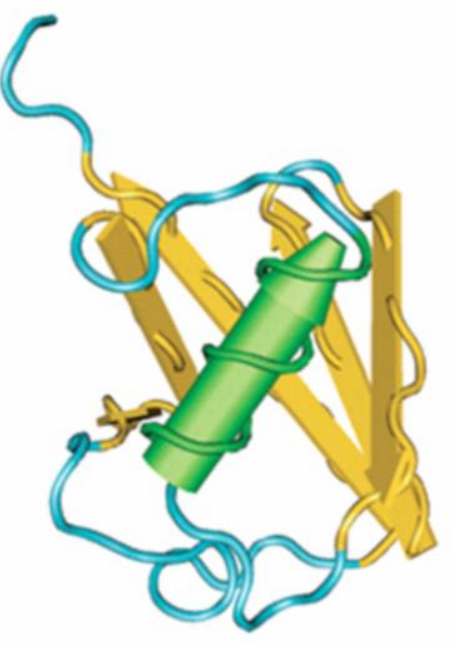

SUMO1

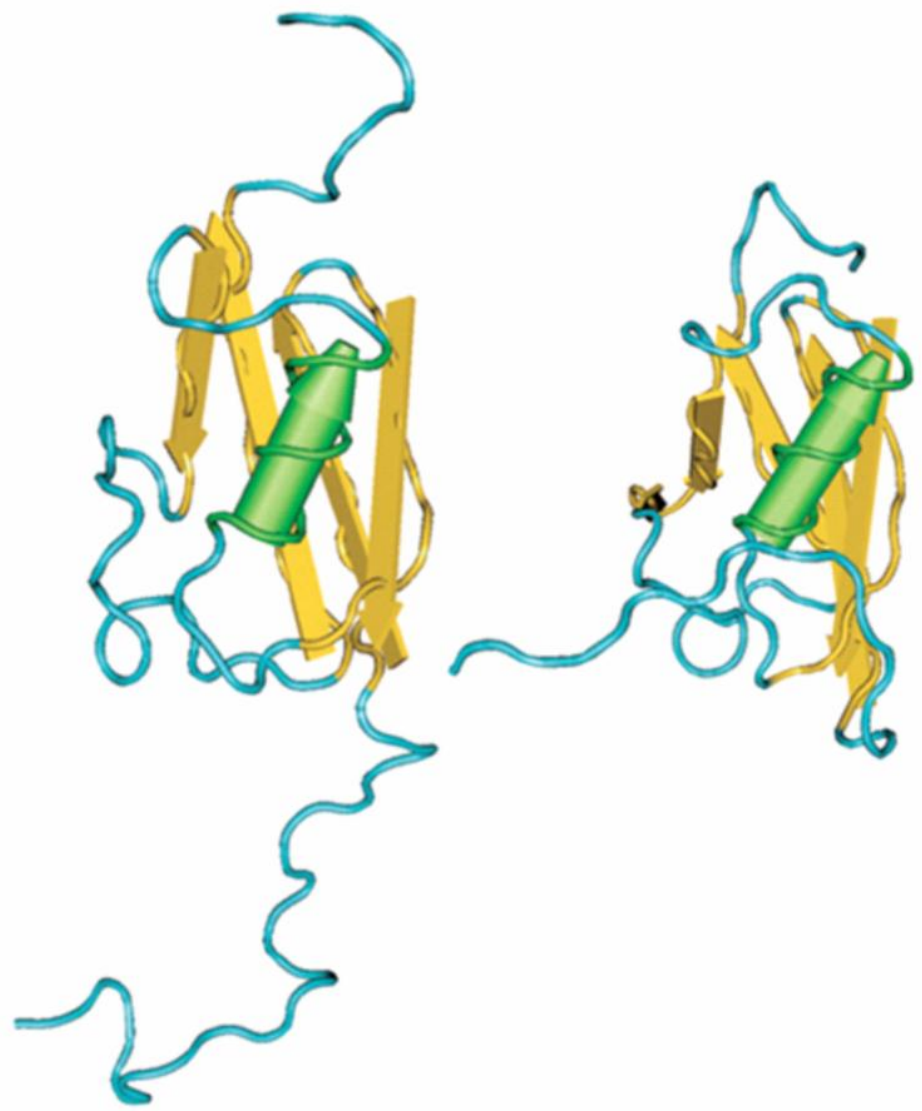

Figure 1-1. Representation of the 3D NMR structures of ubiquitin, SUMO1 and SUMO2/3. Although Ubiquitin and SUMO share just about $20 \%$ amino acid homology, the so called 'ubiquitin fold' $\beta \beta \alpha \beta \beta$ is preserved in SUMO1-3. In contrast to Ubiquitin, SUMO paralogs have long and flexible $\mathrm{N}$-terminal extension. (Martin et al., 2007b)

All SUMO isoforms have a unique flexible $\mathrm{N}$-terminal stretch (10-25 amino acids), that is not found in any other Ubiquitin-related proteins. So far these N-termini have been found to be essential for SUMO chain formation (Tatham et al., 2001). Additionally, SUMO proteins differ from Ubiquitin in their overall surface-charge distribution.

As a common feature of all Ubiquitin-related modifiers, SUMO proteins are synthesized as immature pro-forms having a Gly-Gly C-terminal motif followed by a stretch of amino acids with variable length (2-11 amino acids). Removal of the Cterminal extension and revelation of the conserved Gly-Gly motif is done by a family of SUMO-specific proteases and is a prerequisite for SUMO conjugation to substrates. 


\subsubsection{The SUMOylation Pathway}

Similar to Ubiquitination, SUMOylation is a posttranslational modification which requires formation of an isopeptide bond between the C-terminal Gly residue of SUMO and the $\varepsilon$-amino group of a Lys acceptor in the target protein. Both modifications, Ubiquitination and SUMOylation employ an enzymatic pathway with three classes of enzymes: E1-activating enzyme, E2-conjugase and E3-ligase (Figure 1-2). However, there is no overlap in the enzyme molecules used in SUMOylation and Ubiquitination cascades.

The first step of the SUMO pathway is the activation of the C-terminus of a mature SUMO protein by the E1-activating enzyme, the heterodimer Aos1/Uba2 (Desterro et al., 1999; Gong et al., 1999; Okuma et al., 1999). This reaction is ATP dependent and leads to the formation of SUMO-adenylate conjugate. A thioester bond is formed between the C-terminal carboxyl group of SUMO and the Cys residue in the catalytic pocket of Uba2. Next, SUMO is transferred to the E2-conjugating enzyme Ubc9 (Desterro et al., 1997; Johnson and Blobel, 1997; Lee et al., 1998) in a transesterification reaction, which results in the formation of a thioester bond between the C-terminal carboxyl group of SUMO and the Cys residue of Ubc9.

SUMO is conjugated predominantly to acceptor Lys residues, which are part of a $\Psi-\mathrm{K}-\mathrm{X}$-[D/E] consensus motif, where $\Psi$ can be any large hydrophobic residue (I, $\mathrm{V}$ or $\mathrm{L}), \mathrm{K}$ is the target lysine, $\mathrm{X}$ is any residue and $\mathrm{D} / \mathrm{E}$ is aspartate or glutamate (Rodriguez et al., 2001; Sampson et al., 2001). Such consensus site is unique for SUMOylated targets, since no such sequence was identified for ubiquitinated substrates. A possible reason for this could be that Ubc9 is the single E2-enzyme in the SUMO pathway, identified so far, while there are numerous E2-conjugation enzymes involved in ubiquitination.

The presence of a consensus site within the amino acid sequence of a protein is often not sufficient for its efficient modification. For example, consensus Lys residues, buried in stable helices are not recognized by Ubc9 (Pichler et al., 2005). For an acceptor site to be SUMO modified it needs to be in an extended conformation or part of an unstructured area (Macauley et al., 2006). Amino acid stretches, such as downstream clusters of acidic residues, flanking the consensus site can enhance SUMOylation (Yang et al., 2006). A phosphorylation-dependent SUMOylation motif $\Psi$-K-X-E-X-X-S-P has been reported (Hietakangas et al., 2006), 
where phosphorylation of a Ser residue downstream of the acceptor Lys is a prerequisite for SUMOylation.

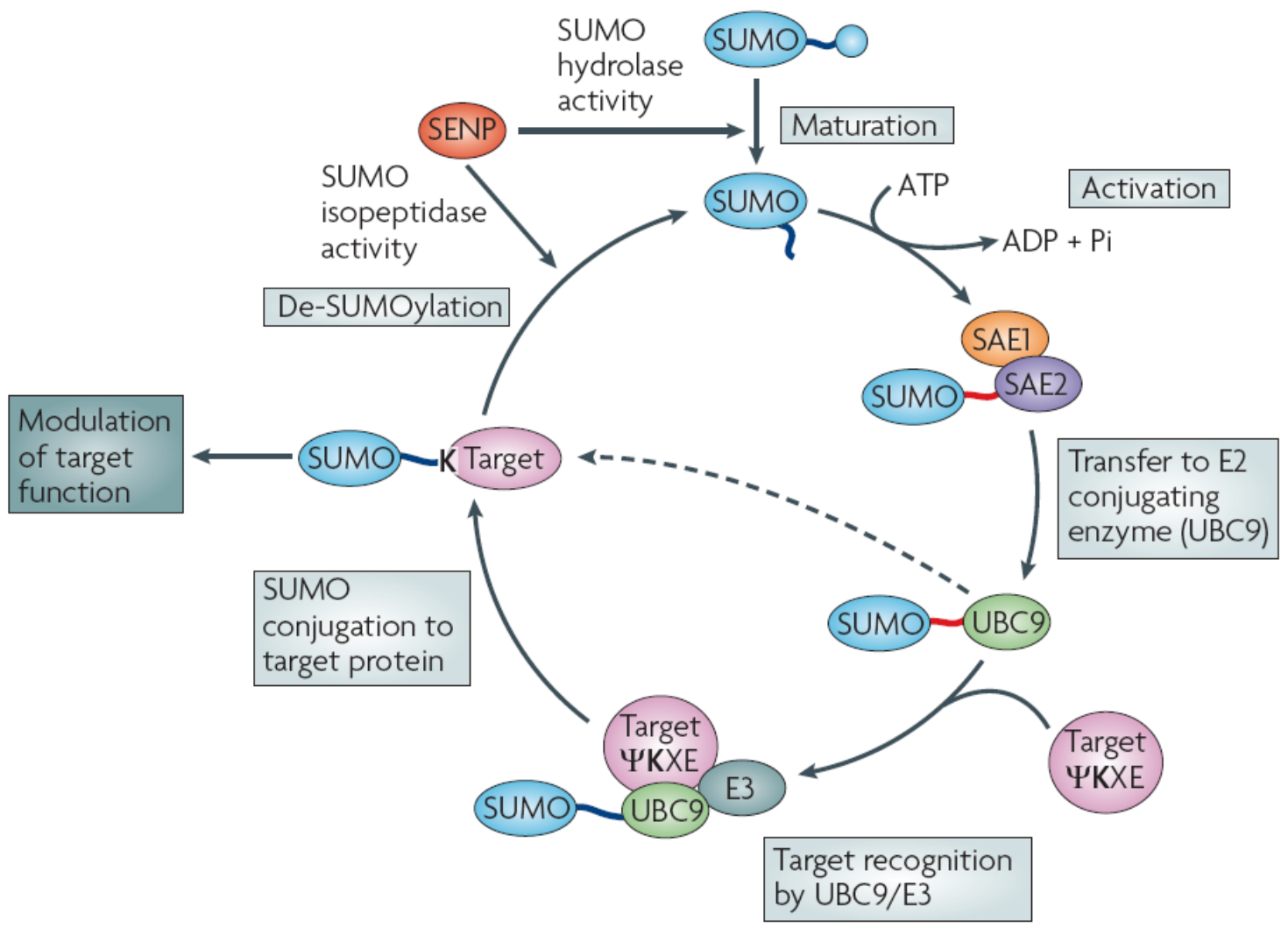

Figure 1-2. The SUMO cycle. Schematic depiction of the SUMO conjugation and deconjugation cascade. All three SUMO isoforms are first synthesized as larger precursors and matured by specific SUMO proteases (SENPs), which reveal the C-terminal Gly-Gly motif of SUMO. The mature SUMO is then activated by the E1- activating enzyme Aos1/Uba2 (SAE1/SAE2) in an ATP dependent process. Activated SUMO is then transferred to the catalytic Cys of E2-conjugating enzyme Ubc9. Some substrates are directly recognized and can be modified only by Ubc9, while others require the activity of E3 ligase for efficient conjugation. Finally, an isopeptide bond is formed between the $\varepsilon$-amino group of the acceptor lysine and the C-terminal carboxyl group of SUMO. SENP family members deconjugate SUMO from substrates. The same molecular mechanism for conjugation and deconjugation applies for all SUMO isoforms. (Martin et al., 2007b)

Ubc9 can recognize and bind directly to consensus SUMO motifs (Sampson et al., 2001). The hydrophobic amino acid side chain, belonging to the consensus motif, has been shown to be in contact with Ubc9 amino acid residues 128-131, while the target Lys is situated in a groove, formed by mainly hydrophobic Ubc9 residues. The $\varepsilon-N$ Lys atom lies within a hydrogen-bond-distance from the catalytic Cys93 of Ubc9, which allows an isopeptide bond formation between the C-terminal Gly of SUMO and the $\varepsilon-\mathrm{NH}_{2}$ group of the acceptor Lys (Bernier-Villamor et al., 2002). 
Although many SUMO targets are conjugated within a consensus site, there is an increasing number of proteins, which are modified at Lys residues which do not fall into a consensus motif, e.g. human E2-25K (Pichler et al., 2005), PCNA (Hoege et al., 2002), axin (Rui et al., 2002), K113 in Smad4 (Lee et al., 2003), potassium channel K2P1 (Rajan et al., 2005). It is currently not known how Ubc9 recognizes non-consensus SUMOylation sites.

A recent study by Knipscheer et al. has shown that Ubc9 auto-SUMOylation can modulate its enzymatic activity. SUMOylated Ubc9 is unable to modify certain substrates (e.g. RanGAP1), but exhibits enhanced conjugation towards substrates containing a SUMO Interacting Motif (SIM), like Sp100 (Knipscheer et al., 2008).

For some substrates, like RanGAP1, Ubc9 is sufficient to conjugate SUMO. Nevertheless, for efficient modification of many targets the action of an E3-ligase, which transfers SUMO from Ubc9 to the acceptor Lys, is required.

There are three groups of E3 ligases described in the SUMO pathway. The largest one, the SP-RING-finger like E3 ligases, function as adaptor proteins that directly bind Ubc9 and the SUMO target. SP-RING E3 ligases bind SUMO noncovalently via the SIMs. Such SUMO E3-ligases are the enzymes from the PIAS family (protein inhibitor of activated signal transducer and activator of transcription) (Johnson and Gupta, 2001; Takahashi et al., 2001; Schmidt and Muller, 2002). In mammals five members of this family have been identified so far: PIAS1 (Kahyo et al., 2001; Nishida and Yasuda, 2002; Schmidt and Muller, 2002), PIAS3 (Nakagawa and Yokosawa, 2002), PIASxa (Nishida and Yasuda, 2002), PIASxß (Schmidt and Muller, 2002) and PIASy (Sachdev et al., 2001). MMS21 (known also as NSE2), which is a part of multimeric a complex involved in DNA repair also belongs to the SP-RING ligases (Andrews et al., 2005; Potts and Yu, 2005; Zhao and Blobel, 2005).

The second group of E3 ligases is represented by Ran binding protein 2 (RanBP2) (Pichler et al., 2002). RanBP2 is part of the nuclear pore complex; it binds stably to Ubc9, but not to targets. This is an E3 ligase unique for the SUMOylation machinery, since it shares no sequence homology with any Ubiquitin E3 and is neither a RING-finger nor a HECT-type E3 ligase (Pichler et al., 2004). RanBP2 is facilitating SUMOylation by placing the Ubc9-SUMO complex in an orientation favourable for an attack by acceptor Lys residue (Pichler et al., 2004; Reverter and Lima, 2005; Tatham et al., 2005). In vivo targets of RanBP2 have not yet been 
identified. However its role as an E3-ligase has been confirmed in vitro for a number of substrates, one of which is Sp100 (Pichler et al., 2002).

Finally, E3 ligase activity has been ascribed to the human polycomb protein 2 (Pc2) (Kagey et al., 2003). The exact mechanism how Pc2 exerts its E3-ligase activity remains unknown.

SUMO1 gets attached to substrates as a single molecule, while SUMO2/3 can form polymeric chains due to the presence of a consensus SUMO motif (VKTE) at the $\mathrm{N}$-terminus of SUMO2/3. Indication for a distinct function of the poly-SUMO2/3 chains comes from chain-deficient SUMO2/3 mutant studies in yeast, showing deficits in chromosome pairing during meiosis (Cheng et al., 2006).

SUMOylation is a reversible and highly dynamic posttranslational modification. A class of enzymes SENPs (sentrin specific proteases) is responsible for reversing the SUMO conjugation. SENPS are SUMO-specific Cys-proteases which cleave the isopeptide bond formed between SUMO and the target. There are six members of the SENP family in humans SENP1-3 and SENP5-7, which deconjugate SUMO paralogs.

SENP enzymes differ in their preference for cleavage of different SUMO isoforms. SENP5 and SENP3 specifically cleave SUMO 2/3 conjugates and are thus possibly involved in SUMO-chains editing (Di Bacco et al., 2006; Gong and Yeh, 2006). Most SENPs can also cleave SUMO C-terminally to reveal the Gly-Gly motif and are thus involved in SUMO maturation. For SENP6 it has been shown in vitro that it can primarily act as a C-terminal SUMO hydrolase (Kim et al., 2000). The majority of the SUMO proteases localize to different nuclear regions - nuclear periphery (Gong et al., 2000), nucleolus (Nishida et al., 2000), nuclear bodies (Gong et al., 2000; Best et al., 2002) and nuclear pore complexes (Zhang et al., 2002), others like SENP6 are found in the cytoplasm (Kim et al., 2000). The subcellular localization of SENPs is different and therefore they target different subsets of substrates for deconjugation.

Although SUMO1 and SUMO2/3 use the same enzymatic conjugation pathway (Tatham et al., 2001), they most probably serve different functions as the different isoforms can be conjugated to different target proteins (Saitoh and Hinchey, 2000; Vertegaal et al., 2006). A subset of substrates can be modified by both SUMO1 and $\mathrm{SUMO} / 3$, but there is an increasing amount of evidence that the regulatory 
mechanisms for SUMO1 and SUMO2/3 conjugation and deconjugation are different. Finally, the pool of non-conjugated SUMO2/3 is much bigger than that of SUMO1.

SUMO modification is an essential process for most eukaryotic organisms except fission yeast. Deletion of the gene coding for the single SUMO paralog leads to severe growth deficiencies in S.pombe but is not lethal (Tanaka et al., 1999). Two SUMO1 knockout studies in mice have shown controversial results and it is still under debate whether individual SUMO isoforms are essential or SUMO1 functions can be compensated by SUMO2/3 and vice versa (Alkuraya et al., 2006; Zhang et al., 2008a). Nevertheless, the abrogation of the SUMO conjugation by Ubc9 knockout causes embryonic lethality in mice, which dispels any doubts that SUMOylation is an essential cellular process (Nacerddine et al., 2005).

\subsubsection{Molecular and functional effects of SUMOylation}

In contrast to poly-Ubiquitination, which is mainly connected to proteasomal degradation of modified substrates, the effects of SUMOylation are extremely diverse. The functional consequence of SUMO conjugation is highly dependent on the substrate being modified and is therefore difficult to be predicted.

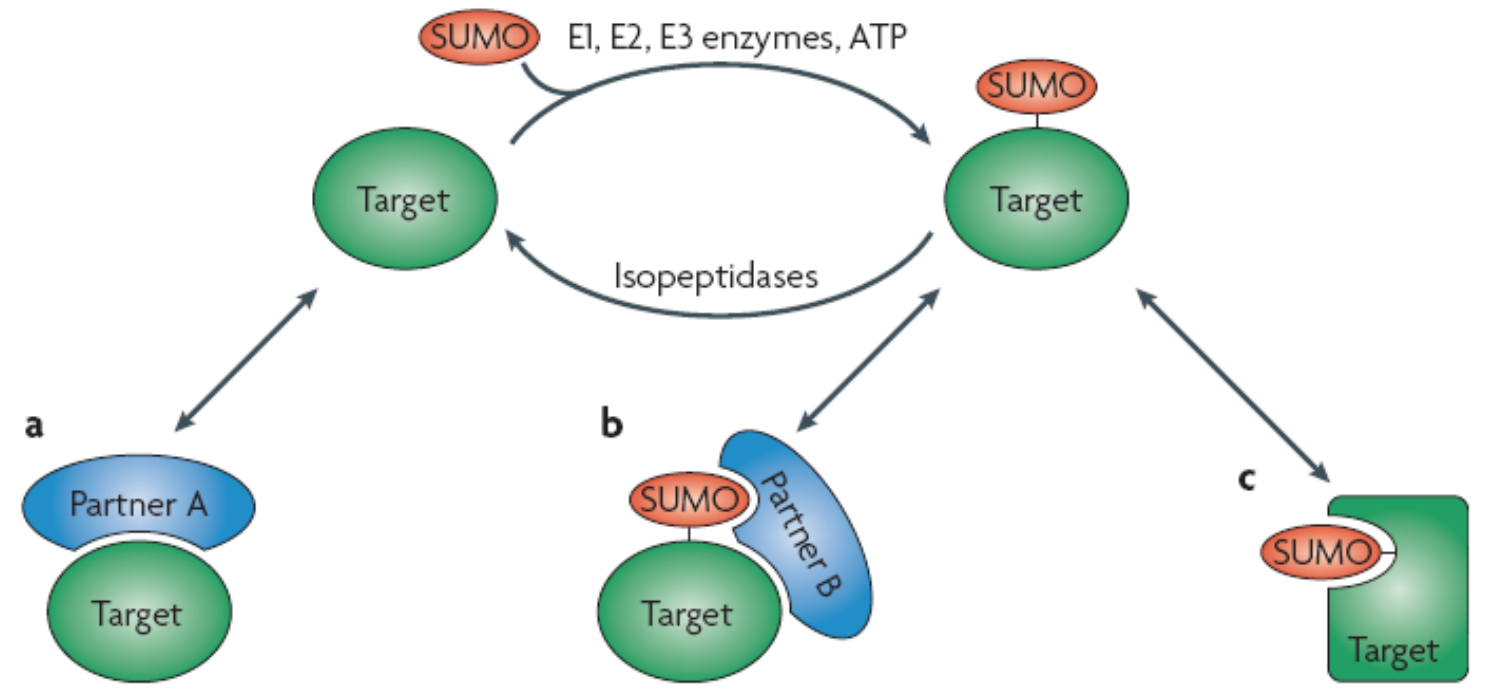

Figure 1-3. Effects of SUMOylation on molecular level. SUMO covalent attachment to a substrate can either block an already existing interaction site (a), create surface for new interactions (b) or lead to structural rearrangements in the modified substrate (c) (Geiss-Friedlander and Melchior, 2007). 
Generally, SUMO attachment to a target protein leads to formation of a new protein interaction surface or masking an already existing one (Figure 1-3). Thus, SUMOylation interferes with protein-protein and protein-DNA interactions and results in a substrate specific fate.

\subsubsection{SUMO in nuclear import and export}

For some proteins SUMOylation leads to a change in their subcellular localization. This has been shown already with the identification of the first SUMOylated target - RanGAP1. Non-modified RanGAP1 is a cytosol resident and only when SUMO gets attached to it, RanGAP1 localizes to the nuclear pore complex (Matunis et al., 1996; Mahajan et al., 1997; Mahajan et al., 1998), where it binds RanBP2. SUMOylation has been considered as a signal for the nuclear localization of many substrates. However, with the increasing number of SUMO targets being identified the actual picture appears to be much more complex.

For substrates like HDAC4 (Kirsh et al., 2002), Sp100 (Sternsdorf et al., 1999) and others mutations in the NLS region abrogates their SUMOylation.

Co-localization studies revealed the association of SENP2 and E2-conjugase Ubc9 to filaments of the NPC (Hang and Dasso, 2002; Zhang et al., 2002). This gives rise to speculations that SUMO conjugation and deconjugation might be coupled to nuclear trafficking. Finally, mutation of the SUMO acceptor site in proteins like the adenoviral type 5 early region $1 \mathrm{~B}(55 \mathrm{kDa})$ oncoprotein prevents its nuclear translocation (Endter et al., 2001), which is a clear indication that SUMO modification is required for nuclear import or necessary for efficient nuclear retain.

\subsubsection{SUMOylation interferes with protein activity}

Covalent SUMO modification has been shown to modulate transcription- factor activity. For most transcription factors, repressive function has been attributed to SUMOylation. Such an example is the GC box binding transcription factor Sp3 for which SUMO1 conjugation leads to relocalization and inactivation (Ross et al., 2002; Sapetschnig et al., 2002). In cases like this, SUMOylation acts as a switch between the active and inactive state of the transcription factor. Few examples have been reported where SUMOylation results in transcriptional activation, as shown for HSF1 and HSF2 where SUMO regulates the DNA-binding ability of the transcription factors (Goodson et al., 2001; Hong et al., 2001). 
Even though many of the reported SUMO substrates have a nuclear localization, other cellular compartments including the plasma membrane are not devoid of SUMO conjugation. SUMOylation has been reported to regulate the inactivation and silencing of the voltage gated Kv1.5 and K2P1 potassium channels (Rajan et al., 2005; Benson et al., 2007).

\subsubsection{SUMOylation induces conformational changes}

Presently there is a small number of structural data for SUMOylated substrates available, which evaluate the effect of SUMO attachment on the target conformation. In contrast to E2-25K and RanGAP1 which display no conformational changes in the SUMO conjugated form (Pichler et al., 2005; Reverter and Lima, 2005), thymine DNA glycosylase $(\mathrm{TdG})$ undergoes structural rearrangements in the $\mathrm{C}$-terminal region upon SUMO1 modification (Baba et al., 2005). TdG is covalently modified by SUMO1 and SUMO3 and additionally has a SUMO Interaction Motif (SIM), via which it interacts non-covalently with either SUMO1 or SUMO3 (Baba et al., 2006). This intermolecular interaction accounts for the conformational change and the subsequent release of DNA in the process of DNA mismatch repair.

\subsubsection{SUMO as a protein solubility enhancer}

Currently SUMO-fusion technology is gaining popularity for recombinant expression and purification of proteins and peptides. $\mathrm{N}$-terminal fusion of SUMO has been shown to change the expression and solubility of the tagged proteins in E.coli (Malakhov et al., 2004). Thus SUMO may play a role in circumventing challenges like proper protein folding and poor solubility of heterologous proteins in bacterial hosts (Butt et al., 2005; Marblestone et al., 2006).

There have been barely any reports on the impact of conjugated SUMO on the target protein solubility and folding. A single study of Shinbo and co-workers shows that Parkinson's disease related L166P mutation in the multifunctional protein DJ-1, leads to improper SUMO conjugation and implicates decreased solubility of the L166P DJ1 mutant (Shinbo et al., 2006).

\subsection{Regulation of SUMOylation}

SUMOylation is a highly dynamic modification and target proteins are being modified upon different stimuli - cell cycle position, stress conditions, DNA damage etc. At a steady state, most SUMOylated proteins represent small fractions of the 
whole protein pool. However due to the conjugation/deconjugation dynamics the entire protein pool can be modified in a relatively short time (Geiss-Friedlander and Melchior, 2007). Tight regulatory mechanisms are required for achieving precise SUMO modification that is localization, time and context dependent.

Posttranslational modifications are in constant interplay and are utilized to influence one another and the function of the modified proteins in the cell. Phosphorylation can serve both as a positive and negative SUMOylation regulator. In the case of p53, phosphorylation of Ser 20 induced by exposure to DNA toxins strongly inhibits SUMOylation (Lin et al., 2004). An opposite effect on SUMO conjugation exerts the phosphorylation of stress-inducible heat shock factor HSF1 (Hietakangas et al., 2003). The phosphorylated Ser residue of HSF1 belongs to a Phosphorylation Dependent SUMOylation Motif (PDSM) and for substrates SUMOylated at Lys residues that fall into this motif phosphorylation is required for efficient SUMO modification (Hietakangas et al., 2006).

Several different posttranslational modifications, such as SUMOylation, Ubiquitination and acetylation, use Lys residues as acceptor sites. In many targets, one and the same Lys has been found to be modified by SUMO and Ubiquitin or acetylated. Such an example is the proliferating cell nuclear antigen PCNA, which has been shown to be SUMOylated or mono- and poly-ubiquitinated on K164 (Stelter and Ulrich, 2003; Papouli et al., 2005). This leads to the assumption that one modification can block another by simply occupying the same acceptor site. However since most targets are not quantitatively SUMOylated or ubiquitinated, but rather a small fraction undergoes certain modification in response to a specific stimulus, it is more likely that SUMOylation and Ubiquitination are independently regulated and trigger different substrate functions.

Regulation of SUMOylation can be achieved not only on substrate level, but by interfering with the molecular players, responsible for SUMO conjugation and deconjugation - the E3-ligases and the isopeptidases.

Different environmental stress factors induce global changes in the SUMO conjugated proteome. Saitoh and co-workers have shown that factors like acute temperatures, osmotic shock and oxidative stress lead to an increase in the levels of SUMO2/3 but not SUMO1 conjugated proteins (Saitoh and Hinchey, 2000). A more profound study by Bossis and Melchior revealed a much more complex regulation of SUMOylation by oxidative stress. Reactive oxygen species (ROS) act on both 
SUMOylation and deSUMOylation enzymes and their effect is dependent on the oxidation levels in the cell. Low physiological concentrations of $\mathrm{H}_{2} \mathrm{O}_{2}$ lead to disulfide bond formation between Ubc9 (E2) and Uba2 (E1) and therefore to global inhibition of SUMO conjugation (Bossis and Melchior, 2006).

Under hypoxic conditions as well as in cerebral ischemia, an elevation of SUMO2/3 conjugation was observed (Yang et al., 2008). Hypoxic stress was also shown to regulate SUMO1 expression on transcriptional level in mice brain and heart tissue (Shao et al., 2004). The exact mechanism of SUMO conjugation induction under sress remains unclear. Recent evidence suggested a role of a RSUME (RING finger and WD repeat-containing SUMOylation enhancer) protein, which directly binds Ubc9, in the increase of overall SUMO conjugation upon hypoxia (CarbiaNagashima et al., 2007).

Altogether these data imply that regulation of SUMOylation on substrate level or globally could be part of the cellular response to various stress factors.

\subsection{SUMOylation as a posttranslational modification in the CNS}

Posttranslational modifications are essential parts of signalling pathways and regulatory mechanisms which enable cells to adapt to the rapidly changing environmental conditions. Their tight control in the central nervous system is of critical importance for maintaining neuronal cell viability, function and connectivity.

For a long time SUMOylation of neuronal specific targets remained unknown, since most assays for identification of SUMO conjugated proteins were carried out in nonneuronal cell lines. The increasing evidence for the involvement of SUMOylation in various cellular processes provoked a number of studies aiming to disclose the role of SUMO in the nervous system. This led to the identification of several intriguing aspects of SUMO conjugation in the CNS.

The first evidence that SUMOylation occurs at the synapse was provided by Martin and co-workers. Modification of the kainate receptor subunit GluR6a induces receptor channel internalization by endocytosis and indicates a role of SUMOylation in modulating synaptic transmission and plasticity (Martin et al., 2007a).

Activity of voltage gated $\mathrm{K}^{+}$channels $\mathrm{Kv} 1.5$ has been shown to be modulated by SUMOylation in myocytes (Benson et al., 2007). The expression of $\mathrm{Kv}$ channels in neurons and the presence of SUMO consensus motifs in Kv1.1 and Kv1.2 potassium 
channels led to the speculation that SUMO might be involved in the regulation of their function in the CNS (Martin et al., 2007b).

SUMOylation was also implicated in axonal mRNA trafficking. The mRNA binding protein La assists local axonal protein synthesis by transporting mRNAs. La has been found to be modified by SUMO and wild type La acts in anterograde and retrograde RNA transport, while SUMOylation deficient La participates only in anterograde transport (van Niekerk et al., 2007).

By modulating the interactions between the cytoskeleton and the extracellular matrix, focal adhesion kinase (FAK) regulates axonal growth and path-finding (IvankovicDikic et al., 2000; Ren et al., 2004). SUMO1 modification of FAK leads to its autophosphorylation and subsequent binding to Src kinases which enables FAK full activation. In the context of FAK SUMOylation influences neuronal cell mobility and axonal guidance (Kadare et al., 2003).

These and studies on other SUMO substrates not mentioned here have suggested diverse roles of SUMOylation in the CNS, ranging from neuronal development and synapse formation to synaptic transmission and plasticity.

\subsection{SUMO in neurodegenerative disorders}

\subsubsection{Polyglutamine disorders}

Polyglutamine diseases are neurodegenerative disorders caused by a trinucleotide expansions (La Spada et al., 1991). They include Huntington's disease (HD), spinobulbar muscular atrophy (SBMA) and spinocerebellar ataxias (SCA) and are characterized with progressive loss of certain neuronal subtypes. The encoded CAG expansions lead to the synthesis of toxic protein species bearing from 30 to more than 300 glutamine repeats and the longer the glutamine stretch, the earlier is the disease onset. By showing an increase in SUMOylated proteins in the cortex of human patients and SCA1 transgenic mice (expressing mutant ataxin-1 with 82 glutamine repeats) Ueda and colleagues for the first time speculated about a potential role of SUMO modification in Polyglutamine disorders (Ueda et al., 2002). Later, ataxin-1 was found to be SUMO conjugated at multiple lysine residues upstream of its polyglutamine tract. Mutant ataxin-1-82Q is to a lesser extent SUMO conjugated than wild type ataxin-1-30Q. Additionally an intact nuclear localization signal (NLS) is needed for efficient SUMO modification of ataxin-1 (Riley et al., 2005). 
Additional indication that SUMOylation could be involved in Polyglutamine toxicity came from the work of Chan et al. in a Drosophila model of spinobulbar muscular atrophy SBMA, where polyQ repeats expand within the androgen receptor $(\mathrm{AR})$ protein. Flies expressing the catalytically inactive SUMO E1-enzyme (Uba2 C175S) in addition to the disease mutant protein ARtrQ112 showed enhanced degeneration, manifested by loss of eye pigment cells (Chan et al., 2002). This suggests that downregulation of SUMOylation has deleterious effect on cells expressing toxic Polyglutamine species.

In 2004 Steffan and co-workers showed for the first time that a protein involved in neurodegeneration is SUMO conjugated. A pathogenic fragment of Huntingtin (Httex1p) was found to be modified by SUMO1 at the N-terminal lysines 6, 10 and 15. SUMOylation led to the increase of transcriptional repression mediated by Htt. Moreover, by generating a SUMO-Htt97QP fusion protein, where SUMO was Nterminally fused to $\mathrm{Htt}$, it has been demonstrated that SUMO reduces $\mathrm{Htt}$ inclusion formation. Huntingtin's cytotoxicity is reduced in flies heterozygous for SUMO and in transgenic Drosophila, expressing SUMO deficient Htt97QP-K6, 10,15R. This indicated that SUMOylation of $\mathrm{Htt}$ contributed to the disease pathology possibly by stabilizing toxic Htt species (Steffan et al., 2004).

\subsubsection{Neuronal intranuclear inclusion disease (NIID)}

Neuronal intranuclear inclusion disease (NIID) is a rare neurodegenerative condition characterized by inclusion formation in neuronal nuclei (Lieberman et al., 1998). Disease pathology includes progressive development of ataxia.

The first report to connect SUMO to NIID showed SUMO1 colocalization with markers for nuclear inclusions in brain autopsy sections from familial NIID cases (Pountney et al., 2003). A study by Takahashi-Fujigasaki et al. confirmed that neuronal intranuclear inclusions of familial and sporadic NIID were immunoreactive with a SUMO1 antibody. Components of the intranuclear inclusion bodies like HDAC 4, PML and Ubiquitin colocalized with SUMO. A major SUMO substrate RanGAP1 was also found associated to the nuclear inclusions in the familial case of NIID (Takahashi-Fujigasaki et al., 2006). The role of SUMO in the inclusion body formation and the disease pathology remains unknown. It has not been shown yet whether free SUMO is being sequestered to the inclusion bodies or some of the components forming the inclusion are SUMOylated prior or after their aggregation. 


\subsubsection{Alzheimer's disease (AD)}

Alzheimer's disease is the most common form of dementia, histopathologically characterized by massive neuronal loss in the cortical and subcortical brain regions. $A D$ is a protein misfolding disease, in which abnormal accumulations of $A B$ and tau are found in the forms of amyloid plaques and neurofibrillary tangles, respectively (Selkoe, 1999). AB is a product of the proteolysis of the transmembrane amyloid precursor protein (APP) by B-secretase (BACE), which cleaves the extracellular domain, and $\gamma$-secretase, cleaving in the membrane spanning region. Many familial AD-mutations in APP and in some members of the $\mathrm{y}$-secretase complex (presinilins) lead to increased $A B$ production (De Strooper and Annaert, 2000).

Although APP was not proven to be SUMO conjugated, two contradicting studies based on SUMO3 overexpression have emerged, implicating a role of SUMOylation in APP proteolytic processing and AB secretion (Li et al., 2003; Dorval et al., 2007). This raises the question whether changes in global levels of SUMOylation by overexpression is a suitable experimental approach to investigate the role of SUMOylation in APP processing. Further studies focusing on the role of SUMOylation for individual targets involved in APP cleavage, like BACE and other secretase factors, are required to answer this question.

Another player in $A D$ is the microtubule-associated protein tau. Hyperphosphorylation is associated with tau self-assembly and formation of intracellular tangles. Tau was shown to be SUMO1 conjugated at Lys340 and blocking of the proteasome increased tau SUMOylation levels. Although, no direct proof that SUMOylated tau is also phosphorylated was provided, the authors observed increase of SUMO1-tau conjugate upon treatment with phosphatase inhibitors and speculated that tau SUMOylation is induced by phosphorylation (Dorval and Fraser, 2006).

\subsection{Synucleinopathies}

Synucleinopathies are a diverse group of neurological disorders characterized by formation of fibrillar $\alpha$-synuclein containing aggregates. They include Parkinson's disease (PD), dementia with Lewy Bodies (DLB) and multiple system atrophy (MSA). 


\subsubsection{Parkinson's disease}

Parkinson's disease is the most common neurodegenerative movement disorder affecting $1 \%$ of the population at 65 years of age and about $5 \%$ of the individuals above 85 years of age. The disorder is characterized by loss of midbrain dopaminergic neurons in the substantia nigra pars compacta (SNpc), which leads to depletion of dopamine in the striatum. This in turn accounts for severe motoric deficits including bradykinesia, resting tremor, rigidity and postural instability. Histological hallmark of PD is the presence of eosinophilic intracytoplasmic inclusions, called Lewy bodies in post-mortem brain sections of PD patients.

The treatments existing nowadays are based on dopamine derivatives and are targeting mainly the disease symptoms and not its cause. In most cases they lead to motoric improvement but fail to stop disease progression.

Table 1. Genes and loci linked to familial PD. (Dohm et al., 2008)

\begin{tabular}{|c|c|c|c|}
\hline Locus/ Inheritance & Gene & Protein function & PD features \\
\hline PARK1 (Ad) & a-synuclein & Synaptic? & $\begin{array}{l}\text { Early onset, lower } \\
\text { prevalence of tremor }\end{array}$ \\
\hline PARK2 (Ar) & Parkin & E3 ubiquitin ligase & $\begin{array}{l}\text { Juvenile onset, } \\
\text { more frequent dystonia, } \\
\text { L-DOPA induced } \\
\text { dyskinesia }\end{array}$ \\
\hline PARK3 (Ad) & Unknown & & \\
\hline PARK4 (Ad) & $\begin{array}{l}\text { a-synuclein } \\
\text { triplication }\end{array}$ & & Rapid progress \\
\hline PARK5 (Ar) & UCHL1 & $\begin{array}{l}\text { Ubiquitin } \\
\text { hydrolase/ligase }\end{array}$ & Typical PD \\
\hline PARK6 (Ar) & PINK1 & Kinase & $\begin{array}{l}\text { Early onset, } \\
\text { slow progression }\end{array}$ \\
\hline PARK7 (Ar) & DJ1 & $\begin{array}{l}\text { Multifunctional } \\
\text { protein/oxidative stress } \\
\text { response }\end{array}$ & $\begin{array}{l}\text { Early onset, } \\
\text { psychiatric symptoms, } \\
\text { slow progression }\end{array}$ \\
\hline PARK8 (Ad) & LRRK2 & & $\begin{array}{l}\text { Juvenile onset, spasticity, } \\
\text { dementia }\end{array}$ \\
\hline PARK9 (Ar) & Unknown & & \\
\hline PARK10 & Unknown & & \\
\hline NR4A2 (Ad) & Nurr1 & & \\
\hline
\end{tabular}


Although $95 \%$ of the PD cases are sporadic, diverse mutations in familial PD patients have given important cues for the molecular mechanism of neurodegeneration leading to disease onset and progression. The gene loci of those mutations, the function of the encoded proteins (if known) and the respective characteristic PD features are outlined in Table 1.

The first link between PD and $\alpha$-synuclein came in 1997 with the identification of PD patients with missense mutations A53T in the SCNA gene (Polymeropoulos et al., 1997) and the demonstration that $\alpha$-synuclein is the major component of the Lewy bodies (Spillantini et al., 1997). Subsequently two other mutations in the synuclein gene locus (SNCA) have been identified: A30P in German kindred (Kruger et al., 1998) and E46K in Spanish kindred (Zarranz et al., 2004). While cases with autosomal dominant mutations of $\alpha$-synuclein are very rare, in more than $90 \%$ of the sporadic PD $\alpha$-synuclein containing Lewy bodies were found.

\subsubsection{Putative functions of $\alpha$-synuclein}

$\alpha$-Synuclein belongs to a family of proteins present in neuronal cytosol and enriched in synapses. Vertebrates have three members of the synuclein family - $\alpha$-, B- and $\mathrm{y}$-synuclein.

a-Synuclein is a soluble small 140 amino acid protein with a highly conserved $\mathrm{N}$-terminus, containing imperfect KTKEGV repeats, a hydrophobic mid-region (NAC non- $A B$ component of $A D$ amyloid) and an acidic carboxy terminus (Figure 1-4). The protein is unstructured in aqueous solutions and its main localization is presynaptic, where it was found associated with synaptic vesicles (Cookson, 2005; Lee and Trojanowski, 2006). The physiological role of $\alpha$-synuclein remains poorly understood. a-Synuclein knock-out mice developed normally without evident neurological and mild electrophysiological and behavioural phenotype (Abeliovich et al., 2000; Cabin et al., 2002) . 


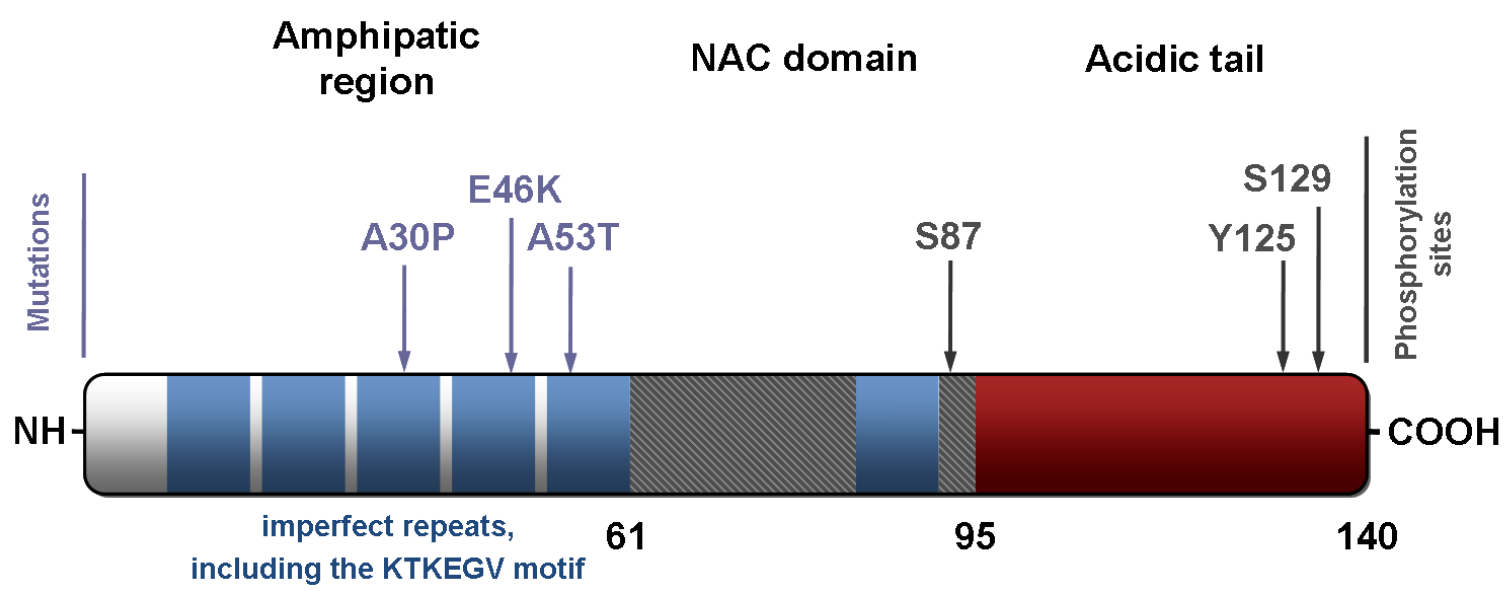

Figure 1-4. Schematic depiction of a-synuclein amino acid sequence. Imperfect repeats, containing the KTKEGV motifs and the C-terminal acidic region are shown in blue and red, respectively. The middle NAC region, prone to aggregation, is illustrated in gray. Autosomal dominant mutations found in familial cases of PD and phosphorylation sites are indicated with arrows. (Adapted from Cookson et al., 2005)

a-Synuclein can bind to lipids and this interaction induces the formation of an amphipatic helix in the N-terminus. This evidence together with the primarily synaptic localization led to the speculation of a synaptic function for a-synuclein. In support of this idea Abeliovich and co-workers showed altered dopamine release and reduction in striatal dopamine levels in synuclein deficient mice.

Co-chaperone function was ascribed to $\alpha$-synuclein as overexpression of $\alpha$ synuclein could rescue the phenotype of cystein-string protein- $\alpha$ (CSP- $\alpha$ ) knockout in mice. CSP- $\alpha$ deletion led to inhibition of SNARE complex assembly, neurodegeneration and lethality. Deletion of $\alpha$ - and B-synuclein intensified these effects (Chandra et al., 2005).

A potential role of $\alpha$-synuclein in vesicular trafficking within the ER-Golgi network was implied. Overexpression of $\alpha$-synuclein is cytotoxic in yeast and one of the first effects observed is obstructed ER-Golgi trafficking. This idea was supported by protective role of the small GTP-ase Rab1, involved in vesicular trafficking, against $\alpha$ synuclein toxicity in other PD models (Cooper et al., 2006).

The functions of the other synuclein family members are equally unclear. Despite significant sequence homology, only a-synuclein contains highly hydrophobic middle region, promoting amyloid formation. This might be the reason why only a-synuclein is able to form fibrillar structures in vitro. A study by Hashimoto et al. in human $\alpha$ - and 
B-synuclein transgenic mice showed that B-synuclein can act as an inhibitory regulator on $\alpha$-synuclein aggregation (Hashimoto et al., 2001).

Finally, a-synuclein knockout animals are lacking PD-like symptoms, indicating that PD is not caused by a-synuclein loss of function. In agreement with these observations, the autosomal dominant inheritance of familial PD indicates a neurotoxic gain of function for a-synuclein.

\subsection{3. $\alpha-S y n u c l e i n$ aggregation and toxicity}

The molecular mechanisms underlying a-synuclein toxicity remain unclear. However, the tendency of $\alpha$-synuclein to fibrilize and aggregate may be crucial for its neurotoxicity. Wild type $\alpha$-synuclein is a highly soluble protein in physiological buffer conditions and its conversion into insoluble fibrillar structures, seen in vitro and found in the Lewy bodies involves structural rearrangements leading to oligomerization and protofibril formation. Protofibrils have the ability to permeabilize membranes and are implicated in apoptosis induction (Volles and Lansbury, 2003). In vitro screening for modifiers of $\alpha$-synuclein fibrillization identified dopamine as stabilizer of $\alpha$-synuclein protofibrils (Conway et al., 2001). ROS generated in the presence of dopamine also increased a-synuclein toxicity (Xu et al., 2002).

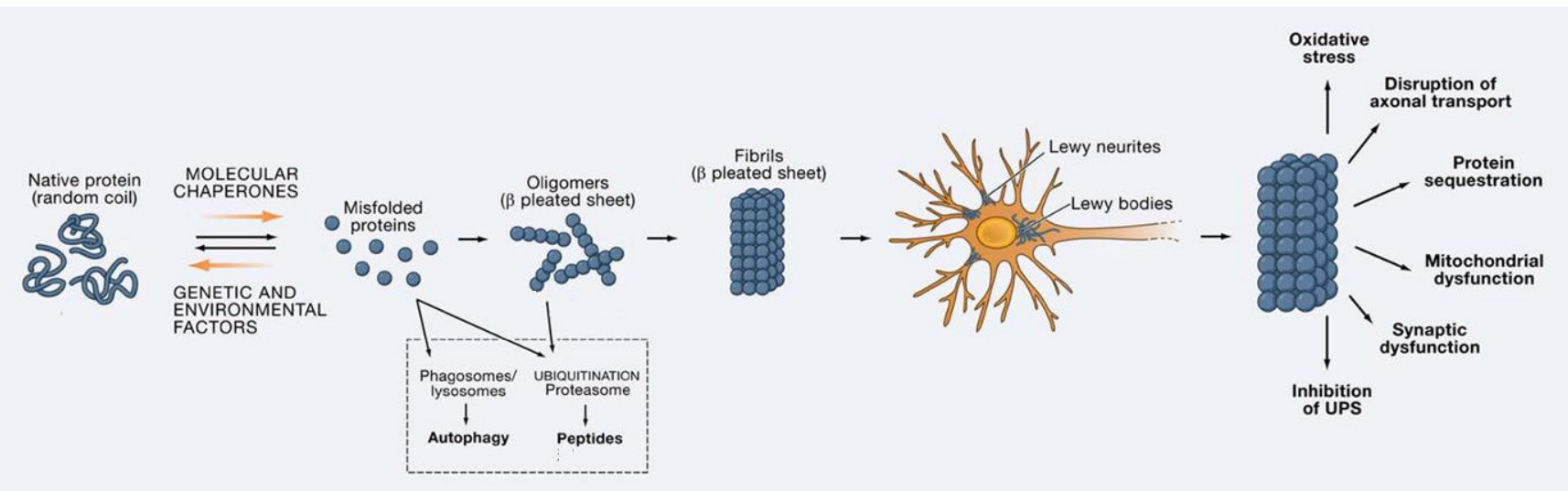

Figure 1-5. a-Synuclein misfolding and aggregation and the downstream consequences. Schematic representation of the steps involved in a-synuclein fibril formation. The highly soluble $\alpha$-synuclein once misfolded can form oligomers and higher order aggregates - amyloid fibrils. The latter are deposited into Lewy bodies and Lewy neurites and might affect various neuronal functions. Genetic mutations and possibly environmental factors (e.g., pesticides), may increase the rate of this process and overwhelm the ability of normal quality-control systems (molecular chaperones, Ubiquitin-proteasomal system (UPS), phagosome/lysosome system) to prevent or reverse protein misfolding or eliminate proteins that have misfolded or assembled into pathological aggregates and amyloid fibrils. Some of the possible effects include 
impaired axonal transport and synaptic function, mitochondrial dysfunction, inhibition of the Ubiquitin-proteasomal system and finally cell death. (Lee and Trojanowski, 2006)

It has been shown by Giasson and co-workers that A53T $\alpha$-synuclein has an increased propensity to form fibrils in vitro and this process is concentration dependent (Giasson et al., 1999). The model proposed by the same group and others implies that the insoluble inclusions would eventually disrupt central cellular functions and lead to cell death (Figure 1-5).

The fact that elevated levels of wild type a-synuclein can be toxic came from the lowan kindred of PD patients, where triplication of the region coding for $\alpha$ synuclein had been identified. Failure in the $\alpha$-synuclein metabolism could be the reason for dopaminergic cell death in the patients from the lowan pedigree and in the idiopathic cases of PD (Gwinn-Hardy et al., 2000; Singleton et al., 2003).

In attempts to explain the selective dopaminergic cell death in PD several animal models have been created.

In Drosophila a-synuclein expression led to the loss of a subset of dopaminergic neurons, which also correlated with fibrillar inclusion formation (Feany and Bender, 2000). One of the first transgenic mice models expressing human $\alpha-$ synuclein developed $\alpha$-synuclein positive inclusions, showed mild motor deficits and dopamine loss in the striatum (Masliah et al., 2000). Studies using viral vectormediated delivery of wild type and mutant $\alpha$-synuclein in the SNpc, demonstrated that dopaminergic neurons are more susceptible to $\alpha$-synuclein toxicity than other subsets of neurons in the nigra (Kirik et al., 2002).

Posttranslational modifications like phosphorylation, Ubiquitination and nitrosylation are additional modulators of $\alpha$-synuclein aggregation and toxicity. The impact of these modifications on the cytotoxic effect of $\alpha$-synuclein is discussed below (Section 1.5.4).

\subsection{4. $\alpha$-Synuclein is subject to various posttranslational modifications}

a-Synuclein undergoes a variety of different posttranslational modifications. One of the first to be described was phosphorylation, which occurs at two major sites Ser 129 and Ser 87. Casein kinase CK-1 and CK-2 were found to be responsible for synuclein phosphorylation at Ser 129 and less efficiently at Ser 87 in PC12 cells (Okochi et al., 2000). All members of the synuclein family have been shown to be substrates for G protein-coupled receptor kinases (GRKs) (Pronin et al., 2000). Ellis 
and others have shown that the Src family of protein tyrosine kinases can phosphorylate a-synuclein at Tyr 125 (Ellis et al., 2001; Nakamura et al., 2002). Later it has been discovered that insoluble $\alpha$-synuclein is extensively phosphorylated at Ser 129 in DLB brain tissue (Fujiwara et al., 2002). Phosphorylation has been shown to modulate toxicity and aggregate formation of a-synuclein also in a Drosophila model of PD. Phosphorylation mimicking S129D mutant of a-synuclein led to an increase in a-synuclein toxicity, while the S129A mutant showed increased aggregate formation but had no toxic effect in flies (Chen and Feany, 2005).

Two recent studies of the impact of Ser129 phosphorylation on dopaminergic cell survival in a rat model of PD showed controversial results (Gorbatyuk et al., 2008; Azeredo da Silveira et al., 2009).

Ubiquitination is the main mechanism targeting proteins for degradation and this modification has also been implied in PD pathology. Ubiquitin is a common constituent of inclusion bodies from diverse neurodegenerative disorders (Lowe et al., 1988) and ubiquitinated $\alpha$-synuclein has been found in cellular inclusions and Lewy bodies of PD patients (Shimura et al., 2001). a-Synuclein is ubiquitinated in vitro and in cell culture at various lysine residues (Lys 21, Lys 23, Lys 32, and Lys 34). Additionally, filamentous a-synuclein can be ubiquitinated in vitro but the modification occurs preferentially at Lys 6, Lys 10 and Lys12 (Nonaka et al., 2005). Several E3 ligases - parkin, SIAH-1 and SIAH-2, have been shown to conjugate Oglycosylated and non-modified synuclein (Shimura et al., 2001; Liani et al., 2004; Lee et al., 2008). Interestingly, mono- and di-ubiquitinated $\alpha$-synuclein by SIAH-1 is not targeted for degradation, but promotes $\alpha$-synuclein aggregation and toxicity (Liani et al., 2004; Lee et al., 2008; Rott et al., 2008).

K63-linked ubiqutin chains have been implicated in a-synuclein S129D inclusion formation in Neuro2a cells (Liu et al., 2007).

It has been shown that $\alpha$-synuclein is degraded by the proteasome (Bennett et al., 1999) but it is still subject of debate whether monomeric a-synuclein needs to be ubiquitinated for its degradation.

Under disease conditions oxidative stress and overproduction of ROS can lead to protein oxidation and ultimately to cell death. Human brain inclusions have been found positive for nitrosylated a-synuclein at all four Tyr residues (Giasson et al., 2000). a-Synuclein is a target for nitration and oxidation. Moreover nitrosylated 
Tyr can be crosslinked and this has been proposed as one of the mechanisms involved in oligomer formation (Souza et al., 2000; Norris et al., 2003)

Recent work has shown that exogenously expressed $\alpha$-synuclein can be modified by SUMO in kidney embryonic 293T cells but failed to identify the conjugation site (Dorval and Fraser, 2006). The effects of SUMOylation on a-synuclein function, aggregation and toxicity remain unknown.

Posttranslational modifications are important regulators of protein function and their dysregulation in the context of $\alpha$-synuclein might have a central role in $\alpha$ synuclein pathology. Further studies on the interplay between different modifications, that $\alpha$-synuclein is subject of, are required to disclose their role in $\alpha$-synuclein misfolding, aggregation and neurotoxicity.

\subsection{SUMOylation in Parkinson's disease}

Very little is known about the role of SUMO in the onset and progression of Parkinson's disease. So far two studies, one on DJ-1 SUMOylation and another on parkin-SUMO interaction, have implicated a role of SUMO in parkinsonian neurodegeneration.

DJ-1 is SUMO modified at $\mathrm{K} 130$ and the conjugation is stimulated by PIASxa and PIASy. Mutation of the SUMO conjugation site disrupted functions like rasdependent transformation and cell growth. Interestingly L166P PD-linked DJ-1 mutation caused multi or poly-SUMOylation of DJ-1. Improper SUMO-conjugation was implicated in decreased DJ-1 solubility and higher susceptibility to UV-induced apoptosis (Shinbo et al., 2006).

Mutations in the PARK2 gene locus coding for the ubiquitin E3 ligase parkin are linked to autosomal recessive juvenile Parkinsonism. A study from Um and Chung has shown that parkin binds SUMO1 non-covalently (Um and Chung, 2006). SUMO 1 interaction with parkin enhances parkin E3 ligase activity.

These observations and the recent evidence that one of the major players in PD a-synuclein can be SUMOylated (Dorval and Fraser, 2006), as well as the potential roles of the SUMOylation machinery in stress response mechanisms (Bossis and Melchior, 2006; Tempe et al., 2008) raise the question what function global SUMOylation and modification of individual targets have in survival and cell death of dopaminergic neurons in PD. 


\subsection{Aim of this study}

The present study had the following aims:

1. To characterize $\alpha$-synuclein as SUMOylation target in vitro, using recombinantly expressed and purified a-synuclein and enzymes of the SUMO conjugation machinery;

2. Subsequent confirmation of $\alpha$-synuclein as a SUMO target in various cell lines;

3. Investigation of $\alpha$-synuclein SUMO-conjugation in brain material from SUMO transgenic mice;

4. Identification of $\alpha$-synuclein SUMOylation site;

5. Characterization of the functional impact of SUMO conjugation for $\alpha$ synuclein;

6. Evaluation of the effects of SUMOylation on subcellular localization, aggregation kinetics and toxicity in cell culture and animal models of Parkinson's disease. 


\section{Materials and Methods}

\subsection{Materials}

\subsubsection{Chemicals}

Applichem (Darmstadt, Germany): 2-Propanol, Acetone, Agarose, Ethanol absolute, Glycine, Guanidine hydrochloride, Imidazol, Nonfat dried milk powder, Potassium chloride $(\mathrm{KCl})$, Sodium chloride $(\mathrm{NaCl})$, Sodium hydroxide pellets, Tris, Tween 20.

BIO-RAD (München, Germany): Dual Protein Standard, Poly-Prep Chromatography Columns (10ml and $25 \mathrm{ml})$.

GE Healthcare (Chalfont St. Gilles, UK): dNTP set (100 mM), $1 \mathrm{ml}$ Mono Q column, PD10 desalting columns, Preparative Superdex75 Chromatography column.

Geneart (Regensburg, Germany): cDNA synthesis and subcloning of a-synuclein $\mathrm{KO} 10$ in pMA vector backbone.

Gibco (Karlsruhe, Germany): B27 Supplement, Neurobasal medium (NBM), OptiMEM.

Greiner Bio One (Frickenhausen): Cell culture dishes, Sterile serological $5 \mathrm{ml}, 10$ $\mathrm{ml}$ and $25 \mathrm{ml}$ pipettes.

Fluka (Steinheim, Germany): Chloral hydrate, Coumaric acid, Polyvinyl alcohol mounting medium with DABCO (antifading), Sodium acetate, Tween 20.

Invitrogen (Karlsruhe, Germany): Lipofectamine 2000, Opti-MEM I Medium, $\mathrm{Ni}^{2+}$ NTA agarose beads, NuPage 4-12\% Bis Tris Gradient Gels 1,5mm, Platinum Pfx

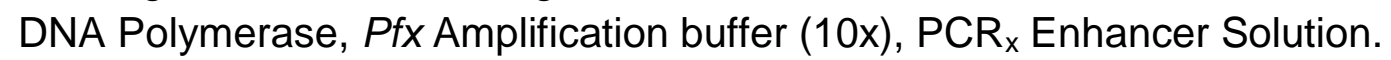

Marchery Nagel (Düren, Germany): NucleoSpin Plasmid kit.

Menzel Gläser (Braunschweig, Germany): SuperFrost Plus Microscope Slides

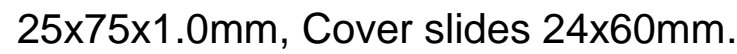

Merck (Darmstadt): Sodium hydrogen carbonate $\left(\mathrm{NaHCO}_{3}\right)$ Ammonium peroxide, Calcium chloride dihydrate, Coomassie brilliant blue G250, DAPI, Hydrogen peroxide $\left(\mathrm{H}_{2} \mathrm{O}_{2}\right)$. 
Millipore (Billericon, USA): Amicon Centrifugal filter devices.

New England Biolabs (Ipswitch, USA): T4 ligase, T4 ligase buffer (10x), DNA restriction enzymes.

PAA (Cölbe, Germany): DMEM, DMEM: F12 (1:1), NGS, FCS, penicillin/streptomycin (PS), Trypsin.

Pierce (Rockford, USA): BCA Protein Assay kit, SuperSignal West Pico Substrate.

QIAGEN (Hilden, Germany): QIAGEN Plasmid Maxi Kit, QIAquick Gel Extraction Kit, QIAGEN PCR Purification Kit.

Roche (Mannheim, Germany): COMPLETE Protease inhibitor cocktail.

Roth (Karlsruhe, Germany): Acetic acid, EDTA, Glycerol, HEPES, Hydrochloric acid, LB Broth, LB Agar, Methanol, Paraformaldehyde, Sodium dodecylsulfate (SDS), Sodium dihydrogenphosphate dehydrate $\left(\mathrm{NaH}_{2} \mathrm{PO}_{4} 2 \mathrm{H}_{2} \mathrm{O}\right)$, Disodium hydrogenphosphate dehydrate $\left(\mathrm{Na}_{2} \mathrm{HPO}_{4} 2 \mathrm{H}_{2} \mathrm{O}\right)$, Rothiphorese, TEMED, Triton X100.

Serva (Heidelberg, Germany): Bromphenol blue sodium salt (BPB).

Sigma (Steinheim, Germany): 2-mercaptoethanol, Ampicillin (Sodium salt) Aprotinin, Dimethyl sulfoxide (DMSO), Dithiothreitol (DTT), Ethidium bromide, lodacetamide, Leupeptin, Luminol, Magnesium chloride $\left(\mathrm{MgCl}_{2}\right)$, MOPS, NEthylmaleimide (NEM), Ovalbumine, Pepstatin, Poly-L-Ornithine, Sepharose, Sodium azide, Sodium bicarbonate $\left(\mathrm{Na}_{2} \mathrm{CO}_{3}\right)$.

Spectrum Labs (Breda, Netherlands): Spectra/Por cellulose ester dyalisis membrane, MWCO of $10 \mathrm{kDa}$.

Whatman (Part of GE Healthcare, UK): Gel blotting paper, ME25 Membrane filter (mixed cellulose ester) $0,45 \mu \mathrm{m}, \varnothing 47 \mathrm{~mm}$.

\subsubsection{Antibodies}

Anti-a-synuclein, clone syn 211, mouse monoclonal (Zymed Laboratories, Karlsruhe, Germany, \#32-8100);

Anti- $\alpha$-synuclein, clone 42, mouse monoclonal (BD Biosciences, Erembodegen, Belgium, \#610787);

Anti-c-Myc, clone 9B11 mouse monoclonal (Cell Signaling, Danvers, USA); 
Anti-c-Myc, clone 9E10 mouse monoclonal, generous gift from Prof. Frauke Melchior;

Anti-SUMO1 (GMP1), mouse monoclonal (Invitrogen, Karlsruhe, Germany, \#332400);

Anti-ß-Tubulin, rabbit polyclonal (Sigma, Steinheim, Germany, \#T3526);

Anti-VMAT 2 (Vesicular Monoamine Transporter 2, C-terminal), rabbit polyclonal (Millipore, Billericon, USA, \#AB1767)

Secondary antibodies for immunofluorescence were Cy2 or Cy3-coupled antimouse or anti-rabbit IgGs (Dianova, Hamburg, Germany), for western blotting HRP-coupled anti-mouse and anti-rabbit IgGs (Santa Cruz Biotechnology, Santa Cruz, USA).

\subsubsection{Plasmids}

a-synuclein-WT in pT7-7 vector, courtesy of the Lansbury laboratory, Harvard Medical School, Cambridge, MA and kindly provided by Prof. Dr. M.Zweckstetter; a-synuclein-WT in AAV2 vector, kindly provided by Dr. S.Kügler;

a-synuclein-K010 in PMA vector, synthesized and subcloned by Geneart, Stuttgart, Germany;

a-synuclein-mCFP in pcDNA3.1, kindly provided by Dr. C.Dohm ; a-synuclein-HECT in pcDNA3.1, kindly provided by Dr. C.Dohm ; pcDNA 3.0 (Invitrogen, Karlsruhe, Germany); pcDNA3.1(+)/myc-His A (Invitrogen, Karlsruhe, Germany); pEGFP (Clontech, Mountain View, USA);

pT-E1E2S1, kindly provided by Prof. F.Melchior;

pEF/myc vectors (Invitrogen, Karlsruhe, Germany); pET28a+ vector (Novagene, Darmstadt, Germany);

His-SUMO1 and His-SUMO2 into pcDNA3.0, kindly provided by Prof. F. Melchior; His-Ubiquitin (x8) into pcDNA3.0, kindly provided by Prof. F. Melchior; YFP-SUMO $\triangle 4$ into CYFP vector (Clontech), kindly provided by Prof. F. Melchior; YFP-SUMO $\Delta 7$ into CYFP vector (Clontech), kindly provided by Prof. F. Melchior;

\subsubsection{Oligonucleotides}

All oligonucleotides were purchased from Sigma-Aldrich (Steinheim, Germany). 


\subsubsection{Oligonucleotides for cloning human $\alpha$-synuclein into $\mathrm{pEF} / \mathrm{myc} / \mathrm{cyto}$ :}

- Syn-Ncol(fw): 5'CCA CCA TGG CCG ATG TAT TCA TGA AAG GAC TTT CAA AG3'

- Syn-Notl(rev): 5'AAA AAG CGG CCG CGG CTTCAG GTT CGT AGT CTT GAT3'

2.1.4.2. Oligonucleotides for cloning a-synuclein mutants via site-directed PCR mutagenesis:

- syn K96R (fw): 5'CA GCA GCC ACT GGC TTT GTC AGA AAG GAC CAG TTG GGC AAG AA 3

- syn K96R (rev): 5'TT CTT GCC CAA CTG GTC CTT TCT GAC AAA GCC AGT GGC TGC TG 3'

- syn K102R (fw): 5'TC AAA AAG GAC CAG TTG GGC AGG AAT GAA GAA GGA GCC CCA CA 3'

- syn K102R (rev): 5'TG TGG GGC TCC TTC TTC ATT CCT GCC CAA CTG GTC CTT TTT GA 3'

- syn DM-K96R, K102R (fw): 5' ACTGGC TTT GTC AGA AAG GAC CAG TTG GGC AGG AAT GAA GAA GGA GCC 3'

- syn DM-K96R, K102R (rev): 5' GGC TCC TTC TTC ATT CTT GCC CAA CTG GTC CTT TTT GAC AAA GCC AGT 3'

- mR1 - K6R, K10R, K12R (fw): 5' ATG GAT GTA TTC ATG AGA GGA CTT TCA AGG GCC AGG GAG GGA GTT GTG GCT GCT 3'

- mR1 - K6R, K10R, K12R (rev): 5' AGC AGC CAC AAC TCC CTC CCT GGC CCT TGA AAG TCC TCT CAT GAA TAC ATC CAT 3'

- mR2 - K21R, K23R (fw): 5' TGC TTC TGC CAC ACC CTG TCT GGT TCT CTC AGC AGC AGC CAC AAC 3'

- mR2 - K21R, K23R (rev): 5' GTT GTG GCT GCT GCT GAG AGA ACC AGA CAG GGT GTG GCA GAA GCA 3'

- mR3 - K32R, K34R(fw): 5' GTG GCA GAA GCA GCA GGA AGG ACA AGA GAG GGT GTT CTC TAT GTA 3'

- mR3 - K32R, K34R (rev): 5' TAC ATA GAG AAC ACC CTC TCT TGT CCT TCC TGC TGC TTC TGC CAC 3'

- mR4-K43R, K45R (fw): 5' GTT CTC TAT GTA GGC TCC AGA ACC AGG GAG GGA GTG GTG CAT GGT 3'

- mR4 - K43R, K45R (rev): 5' ACC ATG CAC CAC TCC CTC CCT GGT TCT GGA GCC TAC ATA GAG AAC 3'

- mR5 - K58R, K60R (fw): 5' GTG GCA ACA GTG GCT GAG AGG ACC AGA GAG CAA GTG ACA AAT GTT 3'

- mR5 - K58R, K60R (fw): 5' AAC ATT TGT CAC TTG CTC TCT GGT CCT CTC AGC CAC TGT TGC CAC 3'

2.1.4.3. Oligonucleotides for cloning $\alpha$-synuclein-KO and $\alpha$-synuclein-DM mutants into pT7-7 bacterial expression vector:

- syn-Ndel (fw): 5'ATA CAT ATG_GAT GTA TTC ATG AAA GGA CTT TCA AAG GC3'

- syn-HindllI (rev): 5'CAA GAC TAT GAA CCT GAA GCC TAA GAA ATA AGC TTA TCG ATG A3' 
2.1.4.4. Oligonucleotides for cloning $\alpha$-synuclein-KO10 and $\alpha$-synuclein-DM mutants into rAAV-2 vector:

- syn-AAV (fw): 5'AAA AAA AAA GCT AGC AA AGATCT A CCG GTC GCC ACC ATG GAT GTA TTC ATG AAA GGA CTT TCA3'

- syn-AAV (rev): 5'AAA AAA AAA TCG ATA AGC TT GCGGCCGC TT AGG CTT CAG GTT CGT AGT CTT GAT ACC C3'

2.1.4.5. Oligonucleotides for mVenus cDNA amplification and cloning of mVenus-SUMO $\Delta 4$ and $m$ Venus-SUMO $\Delta 7$ fusion proteins:

- mVenus-Nhel (fw): 5'C AGA TCG CTA GCG ATG GTG AGC AAG GGC GAG3'

- mVenus-EcoRI (rev): 5'GA ATT CGA CCT TGT ACA GCT CGT CCA3'

\subsubsection{Cell lines and competent cells}

CSM 14.1 - immortalized rat nigrostriatal cell line;

HEK 293, (ATCC, Wesel, Germany);

AAV-293, (Stratagene, La Jolla, USA);

DH5a - chemically competent E.coli strain;

SURE - electrocompetent E. coli strains (Stratagene, La Jolla, USA);

BL21 (DE3) - (Invitrogen, Karlsruhe, Germany).

\subsubsection{Buffers and Solutions}

\subsubsection{Buffers for gel electrophoresis and western blotting}

Electrophoresis buffer: $192 \mathrm{mM}$ Glycine, 0.1\% SDS, $25 \mathrm{mM}$ Tris-HCl, pH 8.3.

Blocking solution for WB: $5 \%$ Milk in TBS-T.

MOPS (20x): 1 M MOPS, $1 \mathrm{M}$ Tris-HCL, 20 mM EDTA, 2\% SDS.

SDS-Sample buffer (2x): $0.125 \mathrm{M}$ Tris-HCl, pH 6.8, 4\% SDS, $0.15 \mathrm{M} \mathrm{DTT,} \mathrm{20 \%}$ Glycerol, 0.01\% Bromphenolblue.

Transfer buffer: $192 \mathrm{mM}$ Glycine, 20\% Methanol, $25 \mathrm{mM}$ Tris-HCl, pH 8.3.

TBS-T: $0.1 \%$ Tween in TBS, pH 7.6 (for WB).

\subsubsection{Colloidal Coomassie Staining Solutions}

Fixing Solution: $40 \%(\mathrm{v} / \mathrm{v})$ ethanol, $10 \%(\mathrm{v} / \mathrm{v})$ acetic acid.

Dye Stock Solution: $0.1 \%(w / v)$ Coomassie Brilliant Blue G250, $2 \%(w / v)$ orthophosphoric acid, $10 \%(\mathrm{w} / \mathrm{v})$ ammonium sulfate.

Destaining Solution: $1 \%$ acetic acid. 


\subsubsection{Buffers and Solutions for immunohistochemistry}

Blocking solution for IHC: $10 \%$ NGS, $0.1 \%$ Triton X-100 in PBS.

Antibody incubation solution: $2 \%$ NGS, $0.1 \%$ Triton $X-100$ in PBS.

Washing buffer for IHC: $0.1 \%$ Triton X-100 in PBS.

DAPI Solution: $2 \mu \mathrm{g} / \mathrm{ml}$ in PBS.

\subsubsection{Chromatography Buffers}

Ion exchange chromatography Buffer A: $25 \mathrm{mM}$ Tris $\mathrm{pH} 7.7$, degassed.

Ion exchange chromatography Buffer B: $25 \mathrm{mM}$ Tris $\mathrm{pH} 7.7,1 \mathrm{M} \mathrm{NaCl}$, degassed.

Gel Filtration Buffer: 50 mM HEPES pH 7.4, 100 mM NaCl, degassed.

\subsubsection{Buffers for Ni-NTA Pulldown of His-tagged proteins}

Lysis buffer for Ni-pulldown: $6 \mathrm{M}$ Guanidinium- $\mathrm{HCl}, 100 \mathrm{mM} \mathrm{NaH} \mathrm{PO}_{4} /$

$\mathrm{Na}_{2} \mathrm{HPO}_{4}, 10 \mathrm{mM}$ Tris-HCl, $\mathrm{pH}$ 8.0, 10 mM Imidazole.

Wash buffer A: $6 \mathrm{M}$ Guanidinium- $\mathrm{HCl}, 100 \mathrm{mM} \mathrm{NaH}_{2} \mathrm{PO}_{4} /$

$\mathrm{Na}_{2} \mathrm{HPO}_{4}, 10 \mathrm{mM}$ Tris-HCl, $\mathrm{pH}$ 8.0, 10 mM Imidazole.

Wash buffer B: $8 \mathrm{M}$ urea, $100 \mathrm{mM} \mathrm{NaH}{ }_{2} \mathrm{PO}_{4} / \mathrm{Na}_{2} \mathrm{HPO}_{4}$,

$10 \mathrm{mM}$ Tris- $\mathrm{HCl}, \mathrm{pH} 8.0,10 \mathrm{mM}$ Imidazole.

Wash buffer C: $8 \mathrm{M}$ urea, $100 \mathrm{mM} \mathrm{NaH}{ }_{2} \mathrm{PO}_{4} / \mathrm{Na}_{2} \mathrm{HPO}_{4}$,

$10 \mathrm{mM}$ Tris- $\mathrm{HCl}$, $\mathrm{pH}$ 6.3, $20 \mathrm{mM}$ Imidazole, Triton-X-100 to $0.2 \%$.

Wash buffer D: $8 \mathrm{M}$ urea, $100 \mathrm{mM} \mathrm{NaH} \mathrm{PO}_{4} / \mathrm{Na}_{2} \mathrm{HPO}_{4}$,

$10 \mathrm{mM}$ Tris- $\mathrm{HCl}, \mathrm{pH} 6.3,10 \mathrm{mM}$ Imidazole, Triton-X-100 to $0.1 \%$.

Wash buffer E: $8 \mathrm{M}$ urea, $100 \mathrm{mM} \mathrm{NaH} \mathrm{PO}_{4} / \mathrm{Na}_{2} \mathrm{HPO}_{4}$,

10 mM Tris- $\mathrm{HCl}, \mathrm{pH}$ 6.3, 10 mM Imidazole.

Elution buffer: $8 \mathrm{M}$ urea, $100 \mathrm{mM} \mathrm{NaH}{ }_{2} \mathrm{PO}_{4} / \mathrm{Na}_{2} \mathrm{HPO}_{4}$, $10 \mathrm{mM}$ Tris- $\mathrm{HCl}, \mathrm{pH} 8.0,250 \mathrm{mM}$ Imidazole.

\subsubsection{In vitro SUMOylation buffers}

Transport buffer (TB): 20 mM HEPES, $\mathrm{pH} 7.3,110 \mathrm{mM}$ potassium acetate, $2 \mathrm{mM}$ magnesium acetate, $1 \mathrm{mM}$ EGTA, $1 \mathrm{mM}$ DTT, $1 \mu \mathrm{g} / \mathrm{ml}$ each of leupeptin, pepstatin and aprotinin.

SUMOylation assay buffer (SAB): $20 \mathrm{mM}$ HEPES pH 7.3, $110 \mathrm{mM}$ potassium acetate, $2 \mathrm{mM}$ magnesium acetate, $1 \mathrm{mM}$ EGTA, $1 \mathrm{mM}$ DTT, 0.05\% Tween 20, 0.2 $\mathrm{mg} / \mathrm{ml}$ ovalbumin, $1 \mu \mathrm{g} / \mathrm{ml}$ each of leupeptin, pepstatin and aprotinin. 
ATP: 100 mM in 20 mM HEPES, pH 7.4, 100 mM magnesium acetate ( $\mathrm{pH}$ adjusted with $\mathrm{NaOH}$ ).

\subsubsection{DNA-calcium phosphate transfection buffers}

Solution A: $250 \mathrm{mM} \mathrm{CaCl}_{2}$ in Millipore water

Solution B: $1.4 \mathrm{mM}$ phosphate (sodium salt of $\mathrm{H}_{2} \mathrm{PO}_{4}^{-}$or $\mathrm{HPO}_{4}^{2-} \mathrm{mM}$ sodium chloride), $50 \mathrm{mM}$ Hepes. The $\mathrm{pH}$ of solution $\mathrm{B}$ was adjusted at room temperature to 7.05 using $\mathrm{NaOH}$ or $\mathrm{HCl}$.

\subsubsection{Other buffers and solutions}

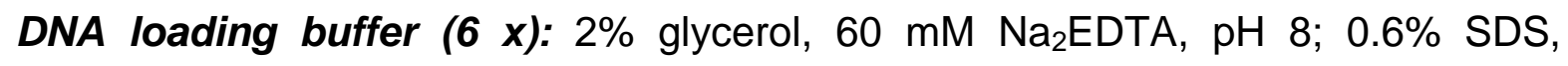
$0.003 \%$ Bromphenolblue.

Lysis buffer (primary rat cortical cell culture): 0.5\% SDS, $1 \mathrm{mM} \mathrm{DTT,} 50 \mathrm{mM}$ Tris- $\mathrm{HCl}, \mathrm{pH} 8.0$.

PBS: $9.55 \mathrm{~g}$ of PBS powder in $1 \mathrm{~L}$ millipore $\mathrm{H}_{2} \mathrm{O}$, autoclaved.

TE: $0.01 \mathrm{M}$ Tris-HCl, $\mathrm{pH} 7.4,1 \mathrm{mM}$ EDTA, pH 8.0.

TBE: 42 mM Boric Acid, 10 mM EDTA, 50 mM Tris-HCl, pH 8.0.

TBS: 150 mM NaCl, 10 mM Tris- $\mathrm{HCl}, \mathrm{pH} 9.0$ (for antigen retrieval).

Trypsin solution (0.25\%) for primary culture: $25 \mathrm{mg}$ Trypsin, $10 \mathrm{ml} \mathrm{CMF.}$

\subsection{Methods}

\subsubsection{Cloning procedures}

Primer design and all major cloning steps were first simulated using GENtle software. Restriction sites, necessary for cloning of the DNA fragments into the respective plasmids, as well as epitope tags insertion was done by PCR using correspondingly designed primers. All basic DNA procedures were performed according to the protocols described in Molecular Cloning Laboratory Manual, $2^{\text {nd }}$ edition (Sambrook et al., 1989).

\subsubsection{PCR - Amplification}

For amplification of the cDNA sequences by PCR several conditions were tested until an optimal result was achieved. Thus, different concentrations (1mM, 2 $\mathrm{mM}$ and $4 \mathrm{mM}$ ) of magnesium sulphate salt $\left(\mathrm{MgSO}_{4}\right)$ in the buffer and annealing temperatures (usually $50^{\circ} \mathrm{C}$ to $65^{\circ} \mathrm{C}$ ) were used for initial amplification. PCR 
reaction mix typically contained: 1x Pfx Amplification Buffer (Invitrogen, Karlsruhe, Germany), $1 \times \mathrm{PCR}_{\mathrm{x}}$ Enhancer Solution (Invitrogen, Karlsruhe, Germany), 1-2 mM of $\mathrm{MgSO}_{4}, 400 \mathrm{nM}$ of sense and antisense primers, $200 \mu \mathrm{M}$ of dATP, dCTP, dGTP, dTTP (GE Healthcare, Chalfont St. Gilles, UK), 10-50ng of the template plasmid DNA, $1 \mu \mathrm{l}$ of the Pfx DNA Polymerase (2,000 units/ml; Invitrogen ,Karlsruhe, Germany), and millipore $\mathrm{H}_{2} 0$ to achieve a total volume of 25 or $50 \mu \mathrm{l}$. The amplification was performed with a Peqlab Cyclone 25 PCR machine (Peqlab Biotechnologies, Erlangen, Germany). The amplification was started with $5 \mathrm{~min}$ initial denaturation at $95^{\circ} \mathrm{C}$, followed by $30-35$ cycles of amplification (annealing $30 \mathrm{sec}$ at $50-60^{\circ} \mathrm{C}$, extension $-1 \mathrm{~min}$ per $\mathrm{kb}$ at $68^{\circ} \mathrm{C}$, melting of DNA strands -30 $\mathrm{sec}$ at $95^{\circ} \mathrm{C}$ ) and final extension of $10 \mathrm{~min}$ at $72^{\circ} \mathrm{C}$. The amplified fragments were separated on $1 \%$ agarose gel by DNA electrophoresis and subsequently purified from the gel using the Gel Extraction Kit (QIAGEN, Hilden, Germany).

\subsubsection{PCR-directed site mutagenesis}

This method uses a proof-reading polymerase to read all the way around a plasmid and thus incorporate a primer as carrier of the new (mutant) sequence in the original plasmid DNA. Only a few (e.g.12) PCR cycles are performed on a large amount of plasmid template to minimize the chance of expanding PCR errors. Two primers were designed, complementary to each other, containing the new (mutant) sequence flanked by 20 bases on each side.

In the PCR reaction most of these primers are annealing to each other, whilst a few are annealing to the target sequence with a small mismatch in the middle. The PCR reaction contained the proof-reading DNA polymerase Pfx (Invitrogen, Karlsruhe, Germany), 10-20 ng template plasmid DNA, $1 \mu \mathrm{M}$ sense and antisense primer, $4 \mu \mathrm{l}$ of $10 \mathrm{mM}$ dNTPs, $2.5 \mu \mathrm{l} \mathrm{Pxf}$ Amplification Buffer (10x), $2.5 \mu \mathrm{l} \mathrm{PCR}$ Enhancer (10x) in a total volume of $25 \mu$ l. The PCR program included: 1) one cycle of initial DNA denaturation for $60 \mathrm{sec}$ at $95^{\circ} \mathrm{C}$; 2) 12 amplification cycles with primer annealing at $55^{\circ} \mathrm{C}$ for $30 \mathrm{sec}$ and extension step at $68^{\circ} \mathrm{C}$ for 2 min per kb of plasmid DNA.

The PCR reaction was cooled to $4^{\circ} \mathrm{C}$ and subjected to Dpnl (New England Biolabs, Ipswich, USA) digest for $1 \mathrm{~h}$ at $37^{\circ} \mathrm{C}$. Dpnl enzyme only cuts dam methylated DNA. The parental plasmid DNA, isolated from a dam+ E.coli strain was digested, while the newly synthesized PCR-DNA was left intact. After restriction 
digest the PCR sample was transformed into chemically competent E.coli. No PCRpurification or ligation was carried out prior the transformation.

The PCR product is a double stranded circular DNA with single stranded nicks in the sense and antisense strands displaced by 42 bp (defined by the primer length). This is identical to a transformation of a classical ligation reaction including a de-phosphorylated vector and an insert.

Transformed E.coli were plated on agar plates with $50 \mu \mathrm{g} / \mathrm{ml}$ Ampicillin and few clones were picked and screened for the mutation by sequencing. The gene of interest, carrying the mutation was then subcloned in the original vector to avoid PCR-errors generated in the vector backbone.

\subsubsection{DNA restriction, electrophoresis, gel extraction, concentration determination}

For restriction digest 5-10 $\mu \mathrm{g}$ of plasmid DNA and 0.5-3 $\mu \mathrm{g}$ of PCR product were used. Appropriate endonucleases in the corresponding buffers (New England Biolabs, Ipswich, USA) were mixed with DNA and digested for 1-2 $\mathrm{h}$ at the temperature specified by manufacturer. Analysis of the DNA size was performed by agarose gel electrophoresis. DNA samples were mixed with DNA loading buffer for loading the gel. The gel was run in $1 \times$ TBE buffer. The DNA bands were visualized by UV-light of $302 \mathrm{~nm}$ at Gel Documentation $2000{ }^{\mathrm{TM}}$ UV-transilluminator (Bio-Rad, Munich, Germany) using the Quantity One software (version 4.2.1). DNA extraction after gel electrophoresis was performed in accordance with QIAquick Gel Extraction Kit (QIAGEN, Hilden, Germany) protocol. The concentration of DNA in the final solution was measured at a Biophotometer (Eppendorf, Hamburg, Germany) at 260 $\mathrm{nm}$.

\subsubsection{DNA ligation and transformation in $E$. coli}

For ligation vector DNA and cDNA fragment were mixed in a molar ratio of 1:1 and 1:3 with T4 DNA ligation buffer (50 mM Tris- $\mathrm{HCl} \mathrm{pH} \mathrm{7.5,} 10 \mathrm{mM} \mathrm{MgCl}_{2}, 10$ $\mathrm{mM}$ dithiothreitol, $1 \mathrm{mM}$ ATP, $25 \mathrm{\mu g} / \mathrm{ml}$ bovine serum albumin, millipore sterile water and T4 DNA ligase ( $1 \mu \mathrm{l}=2000$ units, New England Biolabs) in a total volume of 10 to $20 \mu \mathrm{l}$. The ligation reaction was performed for $1 \mathrm{~h}$ at room temperature. The DNA ligation reaction was transformed into chemically competent $\mathrm{DH} 5 \mathrm{a}$ cells. Defrozen competent cells were incubated with $5-10 \mu \mathrm{l}$ of the ligation reaction for $20 \mathrm{~min}$ on 
ice. The mixture was subjected to heat-shock for $40 \mathrm{sec}$ at $42^{\circ} \mathrm{C}$. Immediately afterwards $1 \mathrm{ml} \mathrm{SOC}$ medium (2\% bacto-tryptone, $0.5 \%$ yeast extract, $10 \mathrm{mM} \mathrm{NaCl}$, $10 \mathrm{mM} \mathrm{KCl}, 20 \mathrm{mM} \mathrm{MgCl}_{2}$ and $2 \mathrm{mM}$ glucose) was added and the transformed cells were incubated for $1 \mathrm{~h}$ at $37^{\circ} \mathrm{C}$ with shaking prior to plating on LB agar plates containing Ampicillin $(50 \mu \mathrm{g} / \mathrm{ml})$ or Kanamycin $(25 \mu \mathrm{g} / \mathrm{ml})$ for clone selections.

The rAAV2 vector DNA ligation products were transformed into electrocompetent SURE cells (Stratagene, La Jolla, USA). The ligation product ( 3 - 5 ng DNA) was added to $50 \mu \mathrm{l}$ of de-frozen and kept on ice electrocompetent SURE E.coli cells. The mixture was transferred to a prechilled on ice cuvette and subjected to an electroporation pulse procedure at Bio-Rad Gene Pulser II (Voltage $=1.8 \mathrm{kV}$, pulse controller-low resistance $=200 \mathrm{Ohm}$, capacitance $=25 \mu \mathrm{F})$, (Bio-Rad, Munich, Germany). Immediately after the pulse cells transferred to a sterile culture tube and $1 \mathrm{ml} \mathrm{SOC}$ medium was added. The transformed cells were incubated for $1 \mathrm{~h}$ with moderate shaking at $37^{\circ} \mathrm{C}$ and then plated on LB agar plates containing Ampicillin $(50 \mu \mathrm{g} / \mathrm{ml})$ for the selection of the clones.

\subsubsection{Plasmid Mini- and Maxi-Preps}

Small- and large-scale DNA plasmid extractions were performed using the QIAGEN Plasmid Mini- and Maxi-kits according to the protocol of the manufacturer (QIAGEN, Hilden, Germany). In brief, the procedure consists of alkaline lysis of the bacterial cell wall, removal of the cell debris while keeping the supernatant containing the nucleic acids, degradation of RNA by RNase, binding of plasmid DNA to a silica-gel matrix and washing with high-salt solution (to remove the chromosomal DNA and proteins) and elution of plasmid DNA.

\subsubsection{DNA Precipitation}

One tenth volume of $3 \mathrm{M}$ Sodium Acetate buffer $(\mathrm{pH}=5.0-5.3)$ was added to the DNA solution to equalize ion concentrations. Two volumes of ice-cold $100 \%$ ethanol were added to the mix, then the mixture was briefly vortexed and left on ice for $5 \mathrm{~min}$. The sample mix was then centrifuged for $20 \mathrm{~min}$ at $16,100 \mathrm{xg}$ in a cooled to $4^{\circ} \mathrm{C}$ centrifuge. The supernatant was removed and $200 \mu \mathrm{l}$ of ice-cold $70 \%$ ethanol were added to the precipitate. The mix was centrifuged again for 10 minutes at $4^{\circ} \mathrm{C}$. After removal of the supernatant, the precipitate was left for drying at room 
temperature for 5-10 min. The DNA pellet was resuspended in desired volume of water or TE buffer.

\subsubsection{Cloning into $\mathrm{pEF} / \mathrm{myc} / \mathrm{cyto}$ vector}

a-Synuclein cDNA was cloned into pEF/myc/cyto vector (Invitrogen) between $\mathrm{Ncol}$ and Notl restriction sites. In this way the encoded fusion protein has a myc epitope tag at the C-terminus.

a-Synuclein cDNA was amplified by PCR from $\alpha$-synuclein-WT in pcDNA3.0. $\mathrm{Ncol}$ and Notl restriction sites were inserted into the primers at the $\mathrm{N}$ - and $\mathrm{C}$ terminus, respectively. The sequences of the oligonucleotides used Syn-Ncol(fw) and Syn-Notl(rev) are listed in Materials section 2.1.4.1.

This plasmid was used for generating the following mutants with Lysine to Arginine substitutions by PCR-directed mutagenesis:

- Synuclein -K96R

- Synuclein K102R

- Synuclein DM - K96R,K102R

- Synuclein mR1 - K6R, K10R, K12R

- Synuclein mR2 - K21R, K23R

- Synuclein mR3 - K32R, K34R

- Synuclein mR4 - K43R, K45R

- Synuclein mR5 - K58R, K60R

- Synuclein KO - K6R, K10R, K12R, K21R, K23R, K32R, K34R, K43R, K45R, K58R, K60R

All oligonucleotides used in the PCR mutagenesis are listed in Materials section 2.1.4.2. 


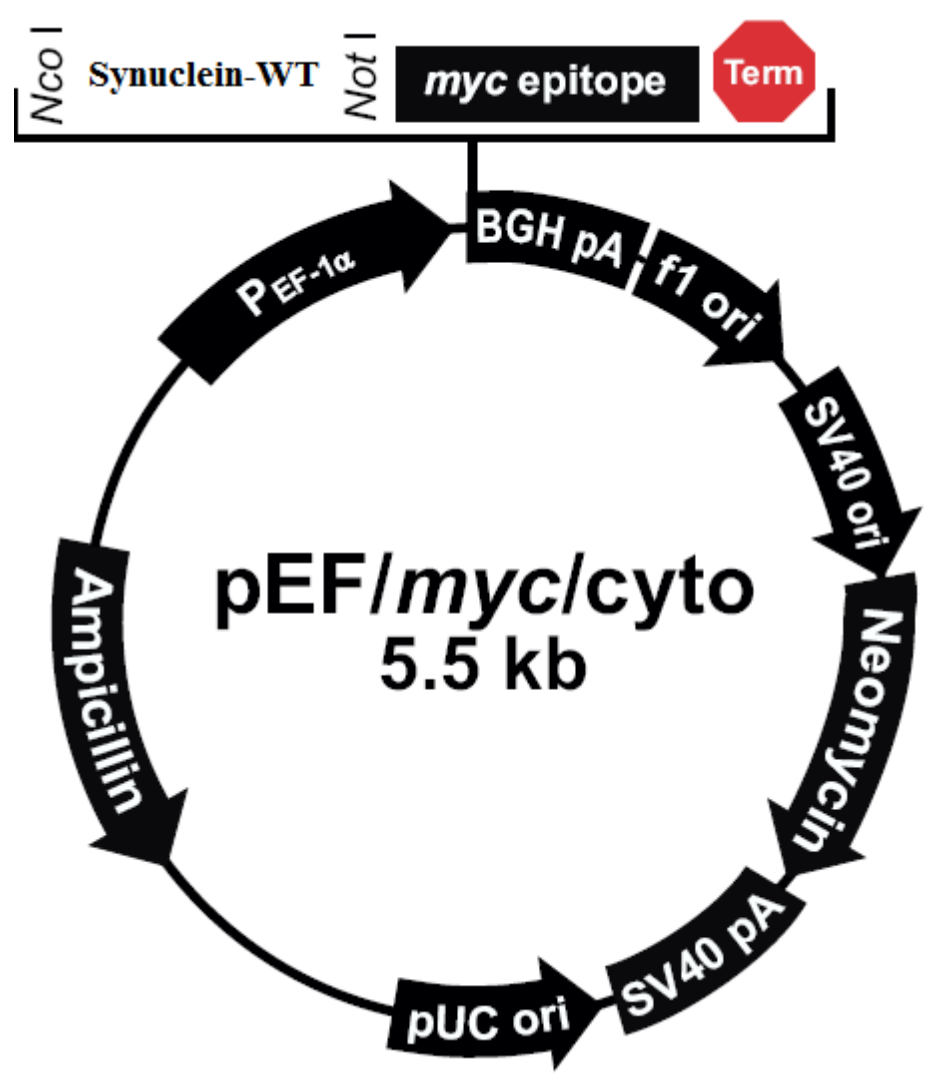

Figure 2-1. Schematic depiction of $\alpha$-synuclein-WT subcloned into $\mathrm{pEF} / \mathrm{myc} / \mathrm{cyto}$ vector.

\subsubsection{Cloning into $p E T 28 a+$ vector}

a-Synuclein-WT cDNA was cut out of $\mathrm{pEF} / \mathrm{myc} / \mathrm{cyto}$ vector with $\mathrm{Ncol}$ and Notl endonucleases (New England Biolabs, Ipswich, USA) and subcloned into pET28a+ (Novagene, Darmstadt, Germany) vector between the Ncol and Notl restriction sites. The plasmid encodes wild type $\alpha$-synuclein with $\mathrm{C}$-terminally fused $6 \mathrm{xHis}$ tag.

\subsubsection{Cloning into pcDNA $3.1 / \mathrm{myc}-$ His vector}

$\alpha$-Synuclein-WT cDNA was excised from $\alpha$-synuclein-HECT in pcDNA3.1 using Hindlll and Xhol restriction enzymes (New England Biolabs, Ipswich, USA) and subcloned into pcDNA3.1/myc-His vector (Invitrogen, Karlsruhe, Germany) with the same restriction sites. The vector has an integrated stop codon downstream of the $6 \mathrm{xHis}$ tag. To allow the expression of the myc and the His epitope tag $\alpha$ synuclein cDNA contained no Stop codon. 


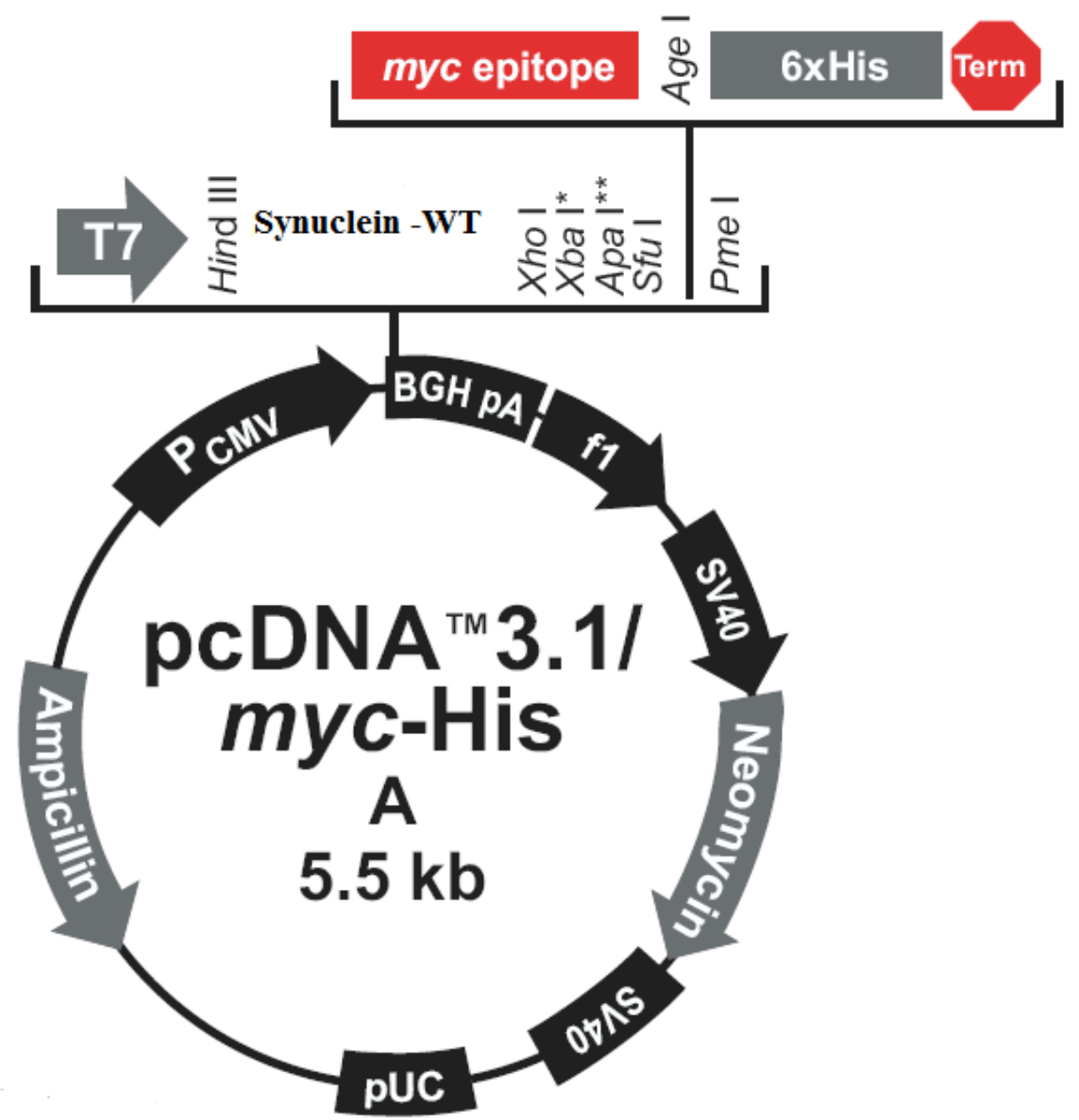

Figure 2-2. Schematic depiction of a-synuclein-WT cloned into pcDNA3.1/myc/His.

\subsubsection{Cloning of mVenus-SUMO1 $\Delta 4$ and mVenus-SUMO1 $\Delta 7$}

Monomeric Venus (mVenus) cDNA was amplified by PCR from mVenus in pcDNA3.0 using mVenus-Nhel (fw) and mVenus-EcoRI (rev) (listed in section 2.1.4.5). Nhel and EcoRI restriction sites were designed in the sense and antisense primer respectively. PCR product was digested with the above mentioned endonucleases as described in section 2.2.1.4. The YFP (Yellow Fluorescent Protein) coding region was cut out of pEYFP-SUMO $\Delta 4$ and pEYFP-SUMO $\Delta 7$ in pEYFP-N1 vector (Clontech, Mountain View, USA) with Nhel and EcoRI restriction enzymes (New England Biolabs, Ipswich, USA) and replaced by mVenus cDNA. 


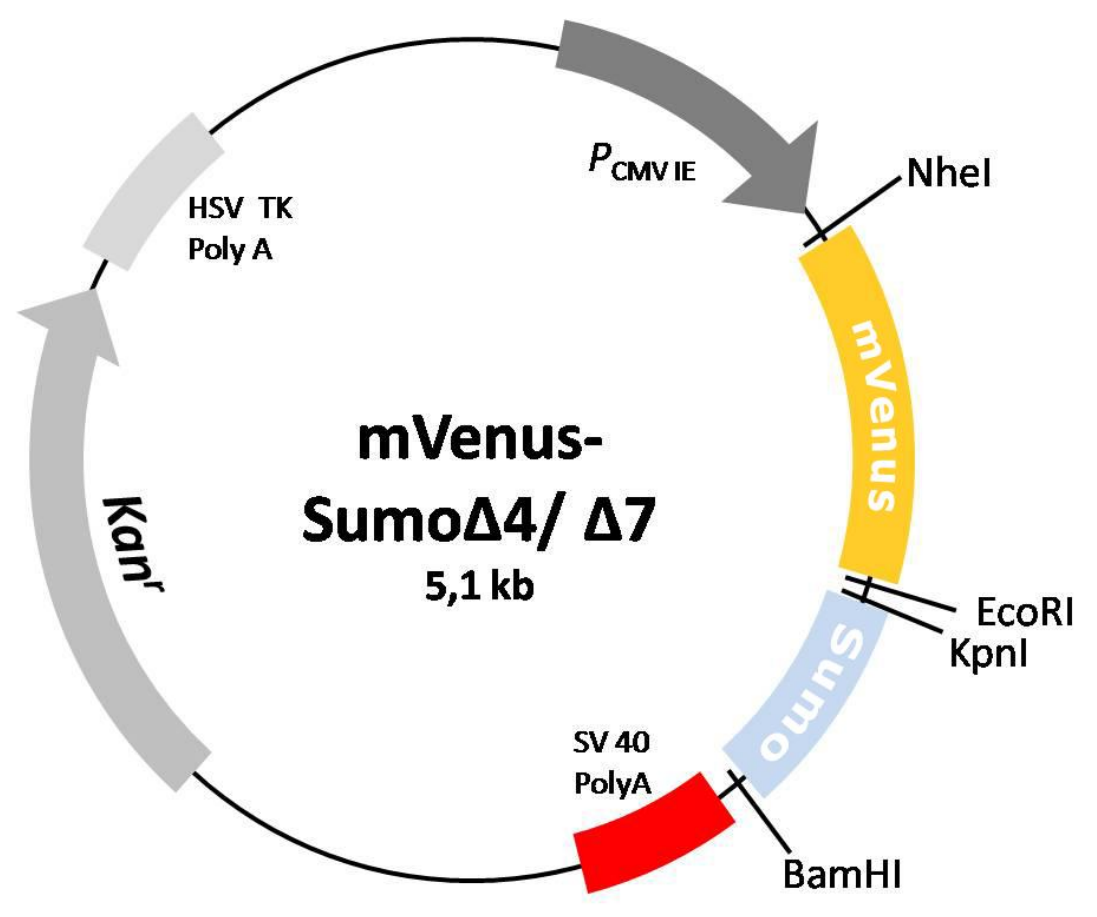

Figure 2-3. Schematic depiction of mVenus-SUMO $\Delta 4 / \Delta 7$ in pEYFP-C1 vector backbone.

\subsubsection{Cloning into pT7-7 vector}

a-Synuclein-WT cDNA subcloned into pT7-7 vector for recombinant protein expression was kindly provided by Prof.Dr.M.Zweckstetter.

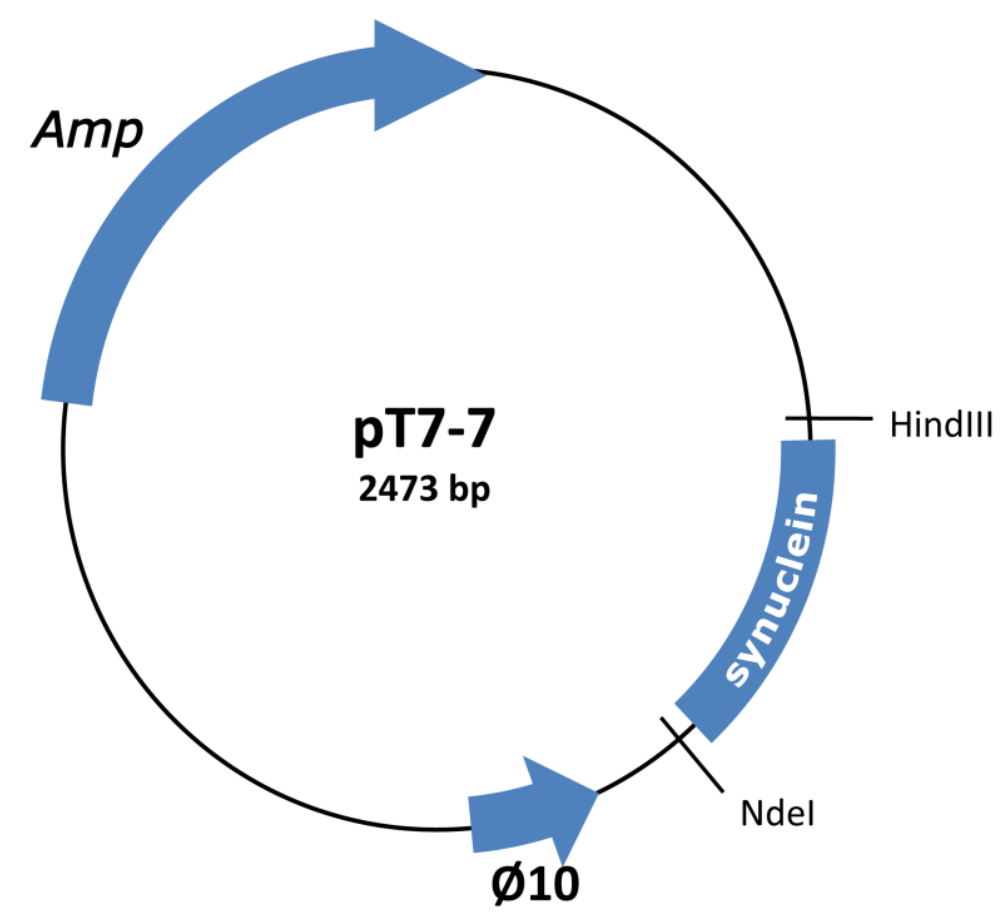

Figure 2-4. Schematic depiction of $\alpha$-synuclein-WT cloned into pT7-7 expression vector; Amp - region encoding Ampicillin resistance; T7 Ø10 promoter. 
For cloning a-synuclein-DM (K96R, K102R) and a-synuclein-KO (K6R, K10R, K12R, K21R, K23R, K32R, K34R, K43R, K45R, K58R, K60R) into pT7-7 vector both cDNA sequences were amplified from $\mathrm{pEF} / \mathrm{myc} / \mathrm{cyto}$ plasmids by using syn$\mathrm{Ndel}(f w)$ and syn-HindIII(rev) primers (listed in section 2.1.4.3). The vector backbone was cut with $\mathrm{Ndel}$ and HindIII and both mutants were inserted using the same restriction enzymes (New England Biolabs, Ipswich, USA).

\subsubsection{Cloning into rAAV-2 plasmid}

Construction of recombinant adeno-associated virus (AAV) vectors has been described previously (Kugler et al., 2003). For this study we employed a vector expressing the functional transgene driven by human synapsin 1 gene promoter. We used pAAV-6p1-TB plasmid (Figure 2-5) as a backbone for the subsequent cloning. The vector backbone was cut with BamHI and Notl (New England Biolabs, Ipswich, USA). a-Synuclein-WT cDNA was amplified from a-synuclein-WT in pT7-7 vector using syn-AAV(fw) and syn-AAV(rev) primers (sequences are listed in Materials section 2.1.4.4). The forward primer was designed including Kozak sequence (Kozak, 1987), which is required for eukaryotic translation initiation, upstream of the ATG Start codon and the BgIll recognition site. The reverse primer included Not/ restriction site. After purification the obtained PCR product was cut by Notl and Bglll restriction enzymes. The compatibility between the ends generated by $\mathrm{BamHI}$ in the vector and $\mathrm{Bg} / \mathrm{Il}$ in the PCR product was made use of, since BamHI recognition site is also internally present in the a-synuclein cDNA. 


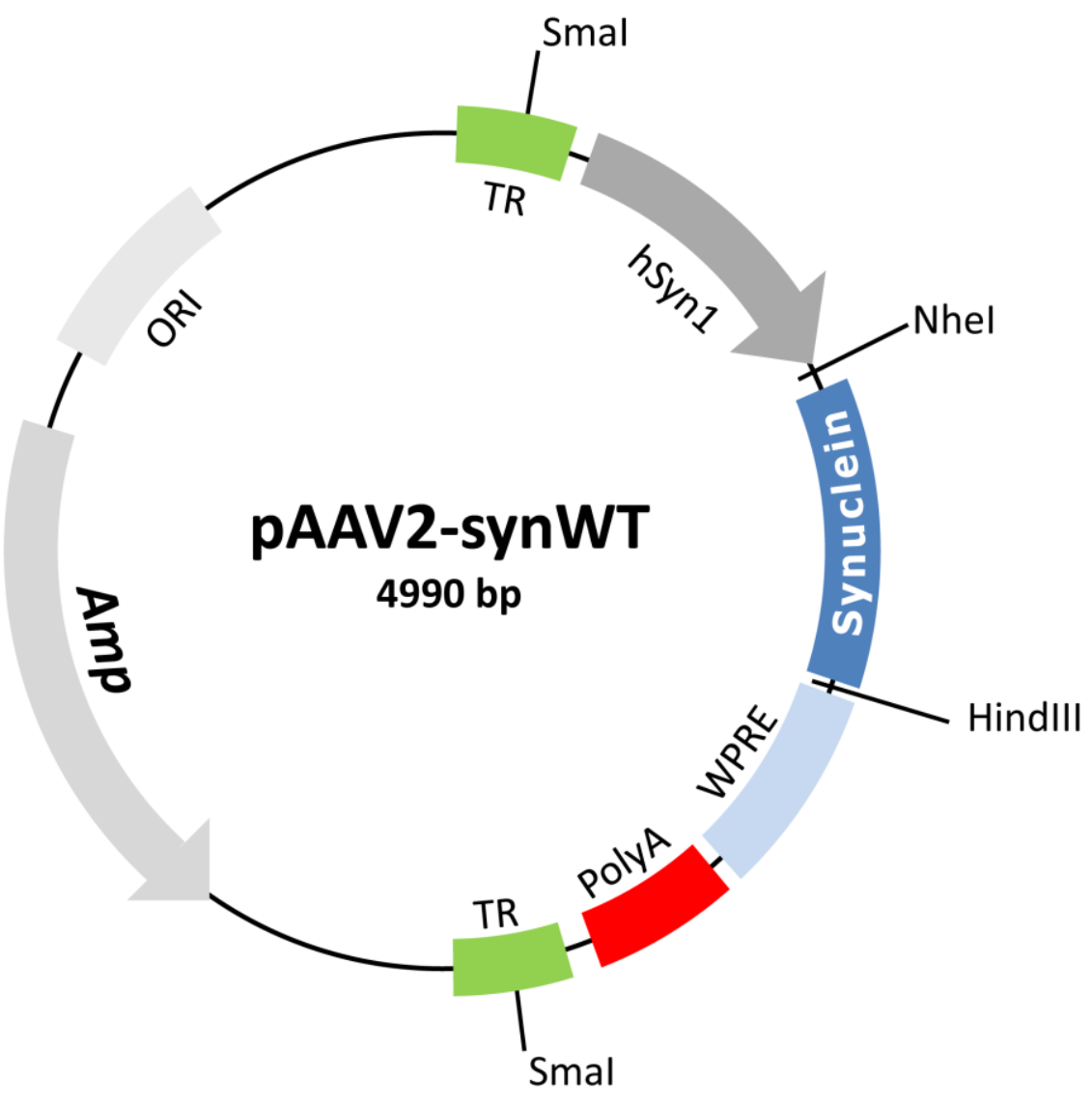

Figure 2-5. Schematic depiction of pAAV2-synuclein. hSYN1, human synapsin-1 gene promoter; WPRE, woodchuck hepatitis post-transcriptional regulatory element; BGH-Poly A - bovine growth hormone polyadenylation signal.

a-Synuclein-DM and $\alpha$-synuclein-KO10 mutant variants were cloned in the pAAV2 vector using the cloning strategy described above. The $\alpha$-synuclein-DM cDNA was amplified from $\alpha$-synuclein-DM in pT7-7 using the same primers as for $\alpha$ synuclein-WT. A-synuclein -KO10 (K10R, K12R, K21R, K23R, K34R, K45R, K60R, K96R, K97R, K102R) cDNA was synthesized and cloned into pMa vector backbone by (Geneart, Regensburg, Germany), (Figure 2-6).

AAV vectors are characterized by high recombination probability due to the presence of inverted terminal repeats (TR). Therefore all AAV plasmids were propagated in the SURE strain of $E$. Coli. The integrity of AAV vector genome was confirmed by a Smal digest (cutting the plasmid in the TR regions) before using the constructs for AAV vector production. 


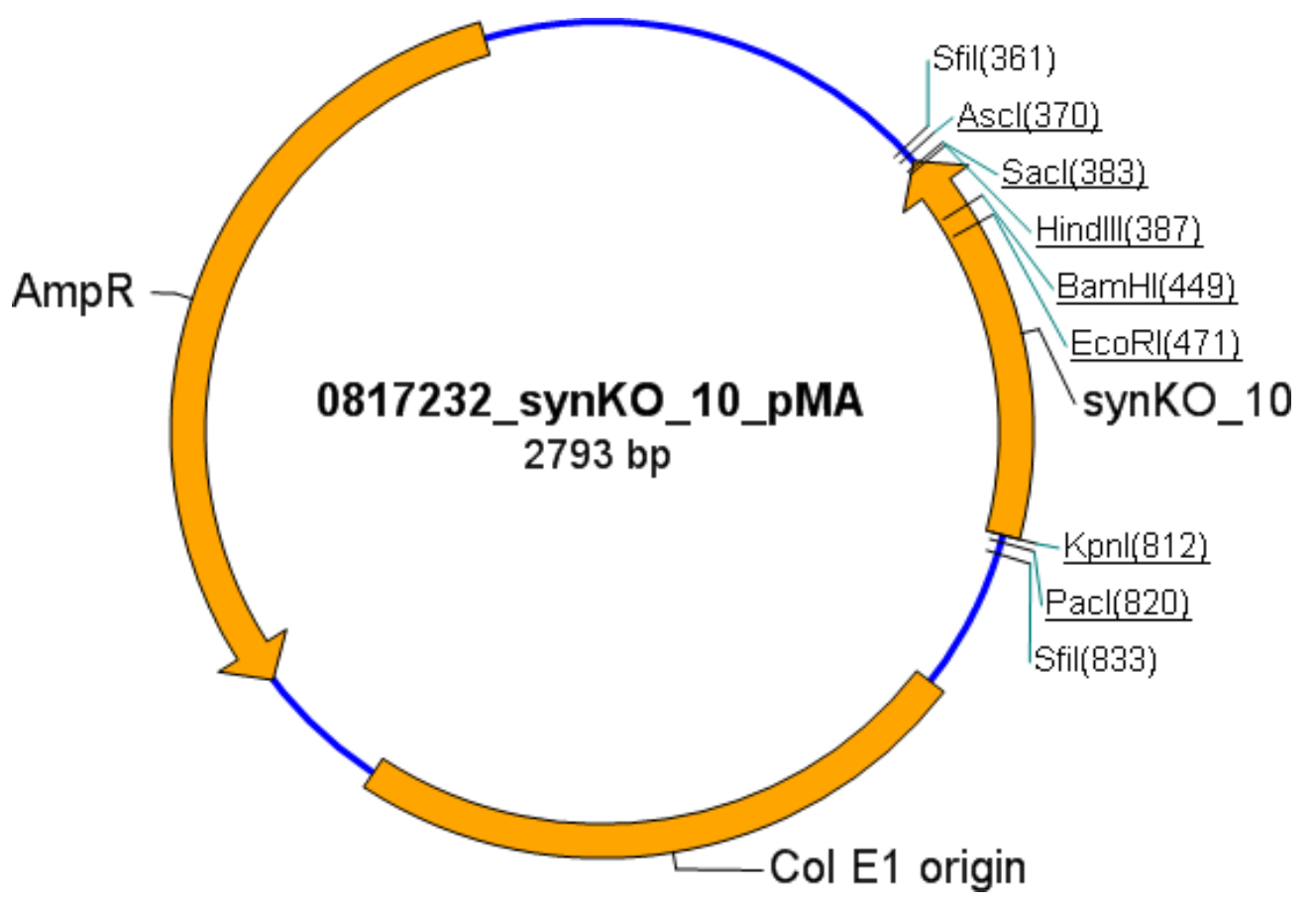

Figure 2-6. Schematic depiction of $\alpha$-synuclein in pMA vector.

Viral vector production and purification was done by Dr. Sebastian Kügler (Neurology Department, S2 Lab, University of Göttingen) and only brief methodology is presented in section 2.2.4.

\subsubsection{Cell culturing}

\subsubsection{Continuous cell culture}

Mammalian HEK 293 were cultured in DMEM supplemented with 10\% FCS and $1 \%$ Penicillin/Streptomycin in $10 \mathrm{~cm}$ culture dishes (Greiner Bio One, Frickenhausen, Germany) at $37^{\circ} \mathrm{C}, 5 \% \mathrm{CO}_{2}$ supply, $95 \%$ humidity. After reaching approximately $70 \%$ confluency, the cells were split 1:4 (every $2-4$ days). For this, the medium was removed, $1 \mathrm{ml}$ of pre-warmed to $37^{\circ} \mathrm{C} 0.05 \%$ trypsin/ $0.02 \%$ EDTA solution were added to the culture and left in the incubator at $37^{\circ} \mathrm{C}$ for $2-3$ min until detached from the flask. The trypsin activity was stopped by addition of $5 \mathrm{ml}$ of the cell culture medium to the flask. The mix containing the detached cells was carefully transferred to a $15 \mathrm{ml}$ Falcon tube and spun down at $400 \times \mathrm{g}, \mathrm{RT}, 4-5 \mathrm{~min}$. After removing the supernatant the cell pellet was resuspended in $40 \mathrm{ml}$ of the fresh medium and seeded in $4(10 \mathrm{~cm})$ tissue culture dishes. 


\subsubsection{Primary culture of cortical neurons}

Coating of 24-well plates (Nunc, Langelselbold, Germany) or coverslips with polyornithine/laminin was done prior to culturing of CNS neurons. For this, ethanolsoaked and flamed coverslips $(\varnothing 10 \mathrm{~mm})$ were incubated with polyornithine solution (500 $\mathrm{\mu l} / \mathrm{well}, 100 \mu \mathrm{g} / \mathrm{ml}$ in millipore sterile water) for $12-24 \mathrm{~h}$ at $\mathrm{RT}$, then washed twice with $0,5 \mathrm{ml} / \mathrm{well}$ of millipore sterile water and coated with laminin $(500 \mu \mathrm{l} / \mathrm{well}$, $1 \mu \mathrm{g} / \mathrm{ml}$ in NBM) by overnight incubation at $37^{\circ} \mathrm{C}, 5 \% \mathrm{CO}_{2}, 95 \%$ humidity. Before the preparation of the cortices, the coverslips were washed twice with NBM and then $500 \mu \mathrm{l}$ of cortex medium (1\% PS-N, $0.5 \mathrm{mM}$ L-glutamine, $0.05 \%$ Transferrin, $2 \%$ B27 Supplement in NBM) was added in each well. To obtain primary cortical neurons, cortices were dissected from Wistar rat embryos (embryonic day 18) and further processed for establishing dissociated cell cultures. All surgical procedures were performed on ice. In brief, cortical tissue pieces were collected in ice-cold cortex medium and centrifuged at $300 \mathrm{xg}$ for $4 \mathrm{~min}$. The medium was removed and the tissue pellet was incubated in $750 \mu \mathrm{l}$ trypsin $\left(0.25 \%, 15 \mathrm{~min}, 37^{\circ} \mathrm{C}\right)$. Trypsin was inactivated by addition of $1 \mathrm{ml}$ ice-cold FCS. The pellet was then dissociated by gentle trituration using a fire-polished Pasteur pipette. After centrifugation at $300 \times \mathrm{g}$ for $4 \mathrm{~min}$, the pellet was resuspended in prewarmed cortex culture medium. Cells were seeded in poly-L-ornithine/laminin 24-well plates at a density 250,000 cells/well. Cultures were maintained in cortex medium at $37^{\circ} \mathrm{C}$ in a humidified atmosphere and $5 \% \mathrm{CO}_{2}$.

\subsubsection{Cell transfection methods}

\subsubsection{Lipofectamine $2000^{\mathrm{TM}}$ transfection}

Transient transfection of adherent cells with plasmid DNA was performed using the Lipofectamine 2000 (Invitrogen, Karlsruhe, Germany) reagent in accordance with manufacturer's instructions. In brief, 30,000 CSM14.1 cells per well were plated in a 24-well plate one day before transfection and grown in DMEM supplemented with 10\% FCS and 1x Penicillin/Streptomycin until 70-80\% confluency. For each transfection sample, DNA-Lipofectamine complexes were prepared by diluting $0.8 \mu \mathrm{g}$ DNA in $50 \mu \mathrm{l}$ of Opti-MEM® I Reduced Serum Medium (Gibco, Karlsruhe, Germany). The appropriate amount of Lipofectamine $(2 \mu \mathrm{l})$ was added to Opti-MEM I Medium (total volume of $50 \mu \mathrm{l}$ ). After 5 minute incubation, the 
diluted DNA was combined with the diluted Lipofectamine (total volume of $100 \mu \mathrm{l}$ ), mixed and incubated for 20 minutes at room temperature to allow the DNALipofectamine complexes to form. The final $100 \mu$ solution, containing DNALipofectamine complexes, was added to each well containing cells and $400 \mu \mathrm{l}$ DMEM medium (without serum and antibiotics). Cells were incubated at $37^{\circ} \mathrm{C}$ and $5 \% \mathrm{CO}_{2}$ for 24-48 hours until they were ready to assay for transgene expression. For transfecting cells in other than 24-well formats DNA concentrations and Lipofectamine amounts were used as given in the manufacturer's instruction.

\subsubsection{DNA-Calcium-Phosphate transfection}

HeLa or HEK 293 cells were transfected using the DNA-calcium phosphate precipitation method. Cells $\left(2 \times 10^{6}\right.$ cells per $10 \mathrm{~cm}$ cell culture dish) were seeded the day before transfection, allowing 60-70\% cell confluency at the time of transfection. Per $10 \mathrm{~cm}$ culture dish 20-30 $\mu \mathrm{g}$ DNA were diluted with $250 \mathrm{mM} \mathrm{CaCl}_{2}$ solution in pure water (Solution $A$ ) to a final volume of $0.5 \mathrm{ml}$. The DNA in solution $A$ was mixed with $0.5 \mathrm{ml}$ of Solution $\mathrm{B}$ (1.4 mM phosphate, $50 \mathrm{mM}$ Hepes). The $\mathrm{pH}$ of solution B was adjusted at room temperature to 7.05 using $\mathrm{NaOH}$ or $\mathrm{HCl}$. The transfection cocktail was vortexed and incubated for $2 \mathrm{~min}$ at RT to allow precipitates to form. The presence of precipitates in the transfection mixture makes it visually more turbid. The turbidity can be measured at wavelengths of $320 \mathrm{~nm}$ or higher, at which neither DNA nor any other components of the mixture absorb. The precipitation mixture $(1 \mathrm{ml})$ was added to $8 \mathrm{ml}$ of pre-warmed to $37^{\circ} \mathrm{C}$ DMEM supplemented with $2 \%$ FCS in which the cells were incubated for $10-12 \mathrm{~h}$ at $37^{\circ} \mathrm{C}$, $5 \% \mathrm{CO}_{2}$. Afterwards transfection medium was changed to DMEM supplemented with 10\% FCS, 1x Penicillin/Streptomycin and cells were grown for additional $24 \mathrm{~h}$ before lysis.

\subsubsection{Viral vector production and purification}

\subsubsection{Virus production}

Viral vectors were propagated in AAV-293 cells using the pDG2 helper, a plasmid encoding the proteins necessary for AAV replication (e.g. capside proteins) (Grimm et al., 1998). Viral particles were purified according to established protocols (Zolotukhin et al., 1999) by iodixanol step gradient centrifugation. Samples were then further purified and concentrated by heparin-affinity chromatography on ÄKTA 
fast protein liquid chromatography system using $1 \mathrm{ml} \mathrm{HiTrap}^{\mathrm{TM}}$ Heparin QFF columns (GE Healthcare, Chalfont St. Gilles, UK) and desalted overnight by dialysis against PBS.

\subsubsection{Virus titration}

Genome titers were determined by quantitative PCR and purity was confirmed by SDS-PAGE. Due to the neuron-specific expression characteristics of the synapsin promoter (Kugler et al., 2001), functional titers of the vectors were confirmed by western blot from the protein expression in cultured primary cortical neurons.

\subsubsection{Identification of SUMOylated targets}

\subsubsection{In vitro SUMOylation using recombinantly expressed proteins}

In vitro SUMOylation assays were performed in a total volume of $20 \mu \mathrm{l}$ in TB (Transport buffer: $110 \mathrm{mM} \mathrm{KOAc,} 20 \mathrm{mM}$ HEPES, pH 7.3, $2 \mathrm{mM} \mathrm{Mg}(\mathrm{OAc})_{2}, 1 \mathrm{mM}$ EGTA, $1 \mathrm{mM}$ DTT, $0.2 \mathrm{mg} / \mathrm{ml}$ BSA, 0.05\% Tween 20) supplemented with protease inhibitors ( $1 \mu \mathrm{g} / \mathrm{ml}$ Aprotinin, $1 \mu \mathrm{g} / \mathrm{ml}$ Leupeptine, $1 \mu \mathrm{g} / \mathrm{ml}$ Pepstatin). The reactions contained $0,5 \mu \mathrm{g}$ recombinant SUMO1 or SUMO2, 0,5 $\mathrm{gg}$ recombinant a-synuclein and low concentrations of recombinant enzymes: E1-activating enzyme (Aos1/Uba2) - 150 ng, E2-conjugase (Ubc9) - 200 ng, E3 ligase - 5-10 ng. Several different E3 ligases were tested in the vitro assays, e.g. PIASy, RanBP2 $\triangle F G$ (a $33 \mathrm{kDa}$ fragment of RanBP2) and IR1+M (catalytically active fragment of RanBP2). Reactions, excluding controls, were incubated with $1 \mathrm{mM} \mathrm{ATP}$ at $30^{\circ} \mathrm{C}$ for $30-60 \mathrm{~min}$ and were stopped by addition of Laemmli-buffer and analysed by SDS-PAGE and Western blot.

Detailed protocols for SUMO enzymes purification are described elsewhere (Werner et al., 2009).

\subsubsection{Identification of SUMOylated proteins using Ni-NTA precipitation}

Uniquitin and ubiquitin-like modifiers (Ubls), like SUMO, are conjugated to target proteins as single molecules or as polymeric chains. These modifications are extremely dynamic and often the steady state levels of the conjugated proteins are low due to the presence of highly active proteases, which deconjugate the ubiquitinlike proteins. Therefore detection of proteins modified by SUMO can be challenging. 
One of the common methods for detecting ubiquitinated and SUMOylated proteins is using $6 \mathrm{xHis}$ tagged Ubls and precipitation with $\mathrm{Ni}^{2+}$-agarose (Jaffray and Hay, 2006).

HeLa or HEK 293 cells (two $10 \mathrm{~cm}$ cell culture dishes per condition) were calcium-phosphate transfected with plasmids for expression of His-tagged SUMO or ubiquitin and the target protein. Thirty-six hours after transfection the cells were harvested and sonicated in guanidinium lysis buffer $(6 \mathrm{M}$ guanidinium $\mathrm{HCl}, 0.1 \mathrm{M}$ $\mathrm{Na}_{2} \mathrm{HPO}_{4} / \mathrm{NaH}_{2} \mathrm{PO}_{4}, 0.01 \mathrm{M}$ Tris- $\mathrm{Cl}, \mathrm{pH}$ 8). Lysates were centrifuged $20 \mathrm{~min}, 4000$ rpm (Heraeus Megafuge 1.0R) for removal of cell debris. $\mathrm{Ni}^{2+}-\mathrm{NTA}$ beads were washed with distilled water and then equilibrated with guanidinium lysis buffer. Histagged proteins were precipitated with $\mathrm{Ni}^{2+}$-NTA beads after 2-3 hours agitation at $4^{\circ} \mathrm{C}$ and subjected to extensive washing with the following buffers:

Wash buffer A: $6 \mathrm{M}$ Guanidinium- $\mathrm{HCl}, 100 \mathrm{mM} \mathrm{NaH}_{2} \mathrm{PO}_{4} /$

$\mathrm{Na}_{2} \mathrm{HPO}_{4}, 10 \mathrm{mM}$ Tris-HCl, pH 8.0, $10 \mathrm{mM}$ Imidazole.

Wash buffer B: $8 \mathrm{M}$ urea, $100 \mathrm{mM} \mathrm{NaH} \mathrm{PO}_{4} / \mathrm{Na}_{2} \mathrm{HPO}_{4}$,

$10 \mathrm{mM}$ Tris- $\mathrm{HCl}, \mathrm{pH}$ 8.0, $10 \mathrm{mM}$ Imidazole.

Wash buffer C: $8 \mathrm{M}$ urea, $100 \mathrm{mM} \mathrm{NaH} \mathrm{PO}_{4} / \mathrm{Na}_{2} \mathrm{HPO}_{4}$,

$10 \mathrm{mM}$ Tris- $\mathrm{HCl}, \mathrm{pH}$ 6.3, $20 \mathrm{mM}$ Imidazole, Triton-X-100 to $0.2 \%$.

Wash buffer D: $8 \mathrm{M}$ urea, $100 \mathrm{mM} \mathrm{NaH} \mathrm{PO}_{4} / \mathrm{Na}_{2} \mathrm{HPO}_{4}$,

$10 \mathrm{mM}$ Tris- $\mathrm{HCl}, \mathrm{pH} 6.3,10 \mathrm{mM}$ Imidazole, Triton-X-100 to $0.1 \%$.

Wash buffer E: $8 \mathrm{M}$ urea, $100 \mathrm{mM} \mathrm{NaH} \mathrm{PO}_{4} / \mathrm{Na}_{2} \mathrm{HPO}_{4}$,

$10 \mathrm{mM}$ Tris- $\mathrm{HCl}, \mathrm{pH}$ 6.3, $10 \mathrm{mM}$ Imidazole.

All buffers were supplemented with $10 \mathrm{mM}$ NEM and Aprotinin, Leupeptin and Pepstatin at concentration of $1 \mu \mathrm{g} / \mathrm{ml}$. Bound proteins were eluted in two fractions of $250 \mu$ Elution buffer ( $8 \mathrm{M}$ urea, $100 \mathrm{mM} \mathrm{NaH} \mathrm{PO}_{4} / \mathrm{Na}_{2} \mathrm{HPO}_{4}, 10 \mathrm{mM}$ Tris- $\mathrm{HCl}, \mathrm{pH}$ 8.0, $250 \mathrm{mM}$ Imidazole). Elution fractions were precipitated using methanolchlorophorm, pellets were air dried and resuspended in 2x Sample buffer. Eluted proteins were separated by SDS-PAGE, transferred onto nitrocellulose membrane, and SUMO or ubiquitin conjugates were detected using target specific antibodies (e.g. anti- $\alpha$-synuclein, anti-myc). Antibody-bound proteins were visualized by horseradish peroxidase-conjugated secondary antibody followed by chemiluminescence (Pierce, Rockford, USA). 


\subsubsection{Identification of in vivo SUMOylated proteins from brain lysates of $\mathrm{His}_{6}$-SUMO2 transgenic mice using Ni-NTA precipitation.}

Mice were decerbrated and their brains were isolated and frozen in liquid nitrogen. Brain material was homogenized in $2 \mathrm{ml}$ Buffer B using glass homogenizer (Braun, Melsung, Germany) on ice. The homogenate was diluted with Buffer B (see 2.2.5.2) to $8 \mathrm{ml}$ final volume and centrifuged $45 \mathrm{~min}$ at $100,000 \times \mathrm{g}$. Supernatant was recovered and centrifuged for additional $15 \mathrm{~min}$ at 100,000 $\mathrm{xg}$ for removal of debris rests. $250 \mu \mathrm{l}$ aliquot was taken and precipitated with methanol-chlorophorm for assessing total protein levels. Brain lysates were incubated with $250 \mu \mathrm{Ni}^{2+}$ agarose on a rotary shaker for $3 \mathrm{~h}$ at $4{ }^{\circ} \mathrm{C} . \mathrm{Ni}^{2+}$-resin was extensively washed with Buffers $B, C, D, E$ (described in section 2.2.5.2). Bound proteins were eluted with $500 \mu \mathrm{l}$ Buffer B supplemented with $250 \mathrm{mM}$ Imidazole (10-15 min shaking at room temperature). After methanol-chloroform precipitation protein pellets were resuspended in $35 \mu \mathrm{l}$ SDS sample buffer, separated on 4-12\% NuPAGE Bis-Tris gradient gels (Invitrogen, Karlsruhe, Germany) and transferred on a nitrocellulose membrane. SUMO conjugates were detected with target specific antibodies (e.g. anti-a-synuclein, Clone 42, BD Biosciences).

\subsubsection{Identification of SUMO conjugation sites by mass spectrometry}

The Detection of $\alpha$-synuclein SUMOylation sites using mass spectrometry was done by He-Hsuan Hsiao and Dr. Henning Urlaub (Bioanalytical Mass Spectrometry Group, Max-Planck-Institute for Biophysical Chemistry).

In brief, SUMOylated $\alpha$-synuclein was cut from 4-12\% NuPAGE Bis-Tris gel and the protein was digested in-gel with trypsin as previously described (Shevchenko et al., 1996). Peptides were extracted and analysed by LC-coupled ESI-MSMS using an orbitrap mass spectrometer (Thermo Fisher Scientific, Waltham, USA). Distinct SUMOylation sites were detected by creating an "artificial" FASTA file with the sequence of $\alpha$-synuclein plus the SUMO1 amino acid sequence (ELGMEEEDVIEVYQEQTGG derived after tryptic digestion of full length SUMO1) incorporated at different sites of the a-synuclein sequence (Urlaub H., Hsiao H., Frank B., programme "chop ' $n$ spice, unpublished). The artificial sequence in FASTA format was implemented into the in-house database and MS and MSMS spectra were searched against the in-house database. The SUMO site was 
detected by matching the MSMS fragment ions against the "artificial" a-synuclein sequence modified with the tryptic SUMO1 peptide.

\subsubsection{Recombinant expression and purification of proteins}

\subsubsection{Recombinant expression and purification of $\alpha$-synuclein}

Wild-type human a-synuclein was expressed in a BL21 DE3 competent $E$. coli (Invitrogen, Karlsruhe, Germany) using a pT7-7 plasmid (courtesy of the Lansbury laboratory, Harvard Medical School, Cambridge, MA, kindly provided by Prof. Dr. M. Zweckstetter). The cells were transformed via heat shock and grown overnight at $37^{\circ} \mathrm{C}$ on LB-agar plates with Ampicillin $(50 \mu \mathrm{g} / \mathrm{ml})$. Clones from the overnight culture plates were picked and placed in $50 \mathrm{ml}$ LB medium containing Ampicillin $(100 \mu \mathrm{g} / \mathrm{ml})$ and were grown overnight at $37^{\circ} \mathrm{C}$. The overnight culture was used to inoculate a larger LB medium volume that was grown to an $\mathrm{OD}_{600} 0.6-0.8$, induced with IPTG (final concentration $1 \mathrm{mM}$ ) and cultured further for four hours at $37^{\circ} \mathrm{C}$. The cells were harvested by centrifugation in a Beckman Avanti J25 centrifuge (JA-10 rotor, $5000 \mathrm{rpm}, 4^{\circ} \mathrm{C}$; Beckman Coulter, Fullerton, USA), resuspended in lysis buffer (10 $\mathrm{mM}$ Tris- $\mathrm{HCl} \mathrm{pH}$ 8.0, $1 \mathrm{mM}$ EDTA) and subjected two consecutive times to emulsion flex (EmulsiFlex C3, Avestin, Mannheim, Germany). The samples were boiled for 20 minutes to precipitate unwanted proteins and centrifuged for 30 minutes (JA-30.50 rotor, 100,000 x g, 4 $\mathrm{C}$; Beckman Coulter, Fullerton, USA). The supernatant was recovered and Streptomycin sulfate was added to a final concentration $10 \mathrm{mg} / \mathrm{ml}$. The sample was stirred for 15 minutes at $4^{\circ} \mathrm{C}$ to allow DNA precipitation, followed by a centrifugation as above. Ammonium sulfate was added gradually to the supernatant to a final concentration of $361 \mathrm{mg} / \mathrm{ml}$ and the mixture was incubated for 20 minutes at $4^{\circ} \mathrm{C}$ to precipitate the $\alpha$-synuclein. The sample was centrifuged as above and the pellet was resuspended in $25 \mathrm{mM}$ Tris- $\mathrm{HCl}(\mathrm{pH}$ 7.7) and dialysed in a Spectra/Por cellulose ester membrane, MWCO of $10 \mathrm{kDa}$ (Spectrum Labs, Breda, Netherlands), overnight at $4{ }^{\circ} \mathrm{C}$ against $4 \mathrm{~L}$ lon exchange chromatography Buffer A. Dialysed sample was concentrated to $1,5 \mathrm{ml}$, filtered (0.2 $\mu \mathrm{m}$ Whatman filter) and loaded onto an ion exchange Mono $Q$ column of a FPLC (GE Healthcare, Chalfont St. Gilles, UK). A salt gradient of $0 \mathrm{mM}-1 \mathrm{M}$ $\mathrm{NaCl}$ was applied and $\alpha$-synuclein was eluted at $\sim 300 \mathrm{mM}$. The elution fractions containing a-synuclein were pooled together and concentrated using AMICON Centriprep columns, MWCO 10,000 Da (Millipore, Billericon, USA). The 
concentrated protein was dialyzed overnight against $4 \mathrm{~L}$ buffer (50 mM HEPES, 100 $\mathrm{mM} \mathrm{NaCl}$ pH7.4) by using 10 kDa MWCO Slide-A-Lyzer dialysis casette (Pierce, Rockford, USA). The same purification procedure was used for purification of $\alpha$ synuclein-DM variant.

\subsubsection{Expression and purification of E.coli SUMO-modified $\alpha$-synuclein}

In vitro SUMOylation of large amount of target protein for further biochemical and biophysical analysis can be rather challenging due to the purification of SUMO conjugating enzymes and the low efficiency of the in vitro SUMOylation reaction for certain proteins. Therefore we used the co-expression of SUMO conjugation enzymes and $\alpha$-synuclein, which led to production of the SUMO -modified protein in E.coli (Uchimura et al., 2004). Possibility for scaling up the experiment and lack of proteases that cleave the covalent bond between SUMO and the target protein are some of the advantages of using this E.coli modification approach. The principal of SUMOylation in prokaryotic cells is depicted in Figure 2-7.
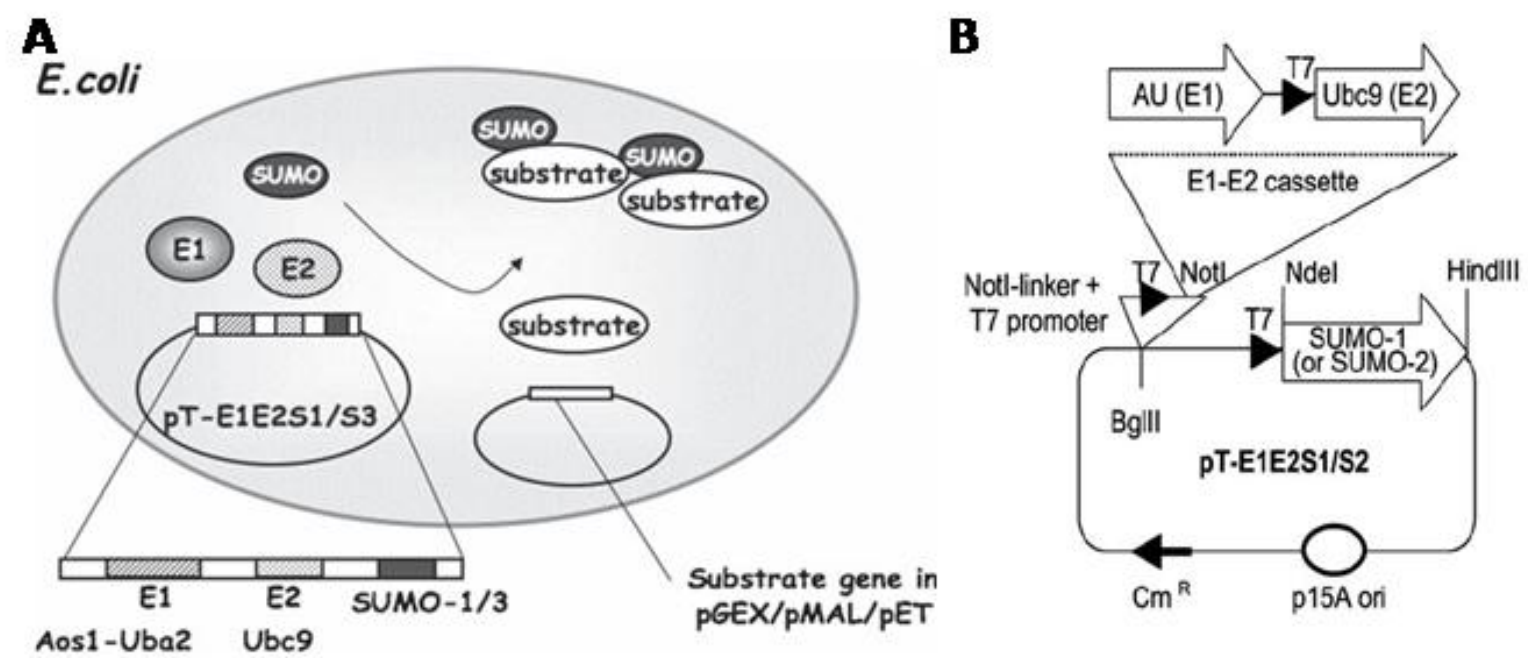

Figure 2-7. Schematic representation of the E.coli SUMO-modification system (A). Polycistronic plasmid containing linear fusion of Aos1 and Uba2 (AU), Ubc9, and SUMO-1-coding regions linked to the T7 promoters. $\mathrm{p} 15 \mathrm{~A}$ origin and the chloramphenicol resistance marker $(\mathrm{CmR})$ are depicted (B). (Uchimura et al., 2004; Saitoh et al., 2009). 
The tri-cistronic plasmid PT-E1E2S1 driving the overexpression of E1- and E2-enzymes and SUMO1 (Fig 2.8) was co-transformed with a-synuclein in pET$28 \mathrm{a}+$ vector in BL21 (DE3) cells.

Cells were grown in SOC medium for $1 \mathrm{~h}$ at $37^{\circ} \mathrm{C}$ with vigorous shaking $(200 \mathrm{rpm}$ in a rotary shaker). $10 \mathrm{ml} \mathrm{LB}$ (supplemented with $25 \mu \mathrm{g} / \mathrm{ml}$ Chloramphenicol, $50 \mu \mathrm{g} / \mathrm{ml}$ Ampicillin, $20 \mathrm{mM}$ Hepes $\mathrm{pH} 7.4,0,05 \%$ glucose and $1 \mathrm{mM} \mathrm{MgCl}$ ) were grown overnight and used to inoculate $2 \mathrm{~L}$ of LB medium. Recombinant protein expression was induced at $\mathrm{OD}_{600} 0,5-0,6$ with IPTG (final concentration $0.5 \mathrm{mM}$ ) and cells were grown for additional $6-8 \mathrm{~h}$ at $30^{\circ} \mathrm{C}$ with reduced agitation speed (150 rpm). Cells were harvested by centrifugation and pellets were resuspended in $150 \mathrm{ml} 50 \mathrm{mM}$ Na-Phosphate (pH 8.0), $300 \mathrm{mM} \mathrm{NaCl}, 10 \mathrm{mM}$ Imidazole, $1 \mathrm{mM} \beta$-Mercaptoethanol, supplemented with protease inhibitors. Bacterial cells were lysed with two passages over emulsion flex, and $100,000 \times \mathrm{g}$ spin for $1 \mathrm{~h}$ at $4^{\circ} \mathrm{C}$. Supernatant was recovered and incubated with $4 \mathrm{ml}$ Nickel beads (Qiagen, Hilden, Germany) for 2-3 h (1 ml beads per liter of culture). Next, beads were excessively washed (20 Column Volumes) with $50 \mathrm{mM} \mathrm{Na-Phosphate} \mathrm{(pH} \mathrm{8.0),} 300 \mathrm{mM} \mathrm{NaCl}, 20 \mathrm{mM}$ Imidazole, 1 $\mathrm{mM} \beta$-Mercaptoethanol. Proteins were eluted with $50 \mathrm{mM}$ Na-Phosphate $(\mathrm{pH} 8.0)$, $300 \mathrm{mM} \mathrm{NaCl}, 200 \mathrm{mM}$ Imidazole, $1 \mathrm{mM} \beta$-Mercaptoethanol. To enrich purity and the amount of SUMOylated protein, all fractions containing SUMO-modified $\alpha$ synuclein were collected, concentrated, desalted on a PD10 column (GE Healthcare, Chalfont St. Gilles, UK) and run onto ion-exchange MonoQ chromatography column.

Elution fractions containing SUMOylated a-synuclein were collected and dialysed against $4 \mathrm{~L}$ Gel filtration buffer (50 mM Hepes pH 7.4, $100 \mathrm{mM} \mathrm{NaCl}$ ) overnight at $4{ }^{\circ} \mathrm{C}$. Dialysed sample was concentrated to $2 \mathrm{ml}$ and further purified using gel filtration (Superdex S75, GE Healthcare).

\subsubsection{Protein handling procedures}

Western Blotting or immunoblotting with a specific primary antibody allows determination of the relative amounts of certain protein present in different samples. Briefly, 1) samples are prepared from recombinantly expressed proteins or tissues or cells that are homogenized in a buffer which protects the protein of interest from degradation; 2) the sample is separated using SDS-PAGE and then transferred to a 
membrane for detection; 3) the membrane is incubated with a generic protein (such as milk proteins) to bind any remaining unoccupied places on the membrane; 4) a primary antibody is then added to the solution which is able to bind to its specific protein; 4) a secondary antibody-HRP conjugate, which recognizes the primary antibody, is added and this allows detection using ECL reagents.

\subsubsection{Protein concentration determination - BCA Assay}

The BCA Protein Assay (Pierce, Rockford, USA) combines the reduction of $\mathrm{Cu}^{2+}$ to $\mathrm{Cu}^{1+}$ by protein in an alkaline medium with the selective colorimetric detection of the cuprous cation $\left(\mathrm{Cu}^{1+}\right)$ by bicinchoninic acid. For the coloured reaction to occur, $\mathrm{BCA}$, a selective colorimetric detection reagent reacts with the cuprous cation $\left(\mathrm{Cu}^{1+}\right)$. The purple-coloured reaction product is formed by the chelation of two molecules of BCA with one cuprous ion. The BCA/copper complex is water-soluble and exhibits a strong linear absorbance at $562 \mathrm{~nm}$ with increasing protein concentrations. The concentration detection range lies between $20 \mu \mathrm{g} / \mathrm{ml}$ and $2 \mathrm{mg} / \mathrm{ml}$.

Reactions were performed in a 96-well plate at room temperature following the manufacturer's instruction. Solution A and B of the BCA Assay kit were mixed 1:50 and the needed number of wells in a 96-well plate were filled, each with $200 \mu \mathrm{l}$ of the mixed solution. Protein standards were prepared in the same buffer in which the unknown samples were in a concentration range between $0.1-1.4 \mathrm{mg} / \mathrm{ml}$. Unknown protein samples were diluted to $1: 2,1: 10$ and $1: 100$ and $1 \mu$ of the dilution was added to the BCA solution A and B mixture. After incubation of 30 min at $37^{\circ} \mathrm{C}$ the absorption of the samples at $562 \mathrm{~nm}$ was measured on Elisa reader TECAN Rainbow using the easyWINbasic software (TECAN, Crailsheim, Germany). Plotting of the absorbance vs. the protein concentration for protein standards to obtain a standard curve and calculation of the unknown samples protein concentration was done using the Microsoft Office 2003 Excel software.

\subsubsection{SDS-polyacrylamide gel electrophoresis (SDS-PAGE)}

SDS-Polyacrylamide Gel Electrophoresis (SDS-PAGE) is based on separation of a large range of proteins of varying molecular weights and charges under the influence of the electrical field within the continuous cross-linked matrix polyacrylamide/ bis-acrylamide (Rothiphorese). Polymer cross-linking is catalysed by free radicals produced upon addition of ammonium peroxide and TEMED. Two- 
phase gels were used for collection and separation of the proteins according to their molecular weight. Usually, $12 \%$ resolving gel and 5\% stacking gel were used to separate the proteins of interest. To define a molecular weight of loaded proteins the molecular weight marker (Dual, Bio-Rad, Munich, Germany) was loaded and separated in parallel. The protein concentration in the samples was adjusted by dilution of more concentrated samples in an appropriate buffer. Equal volumes of the protein samples and $2 \times$ SDS-loading buffer were mixed, subjected to heating at $95^{\circ} \mathrm{C}$ for $5 \mathrm{~min}$, cooled down to room temperature and loaded in the gel combs. SDS-PAGE was run at $4^{\circ} \mathrm{C}$ in ice-cold Tris-Glycine electrophoresis buffer. Electrophoresis was performed using Mini-Protean 3 chamber and Power Pac 300, purchased from Bio-Rad. Electric field of $80 \mathrm{~V}$ was applied for 15 min to allow samples to enter the gel without a smearing and then increased to $100 \mathrm{~V}$ and kept constant until the bromphenol blue reached the bottom of the resolving gel.

Gradient NuPAGE Bis-Tris 4-12\% (Invitrogen) gels were run using MOPS buffer (Invitrogen) in XCell SureLock Mini-Cell electrophoresis chamber (Invitrogen, Karlsruhe, Germany) and proteins were transferred on a nitrocellulose membrane at $4^{\circ} \mathrm{C}$ using Bio-Rad blotting device.

\subsubsection{Immune blotting}

For the western blot procedure a Mini Trans-Blot Cell setup (Bio-Rad, Munich, Germany) was employed. After electrophoresis completion the polyacrylamide gel was placed between two sheets of Whatman filter paper and a nitrocellulose membrane, all preliminary soaked in transfer buffer. A voltage of 100 $\checkmark$ was applied for 60 to $90 \mathrm{~min}$ at $4^{\circ} \mathrm{C}$.

After the completion of the transfer the membrane was treated with blocking solution for $1 \mathrm{~h}$ at room temperature to avoid unspecific binding of the antibody. Incubation with primary antibodies was performed in 5\% Milk/TBS-T solution overnight at $4{ }^{\circ} \mathrm{C}$. The membrane was washed $3 \times 10 \mathrm{~min}$ with TBS-T and incubated for $1 \mathrm{~h}$ at room temperature with secondary antibody. After secondary antibody incubation the membrane was again washed $3 \times 10 \mathrm{~min}$ with TBS-T. The following dilutions of the primary antibodies were used: anti-myc 9E10 (affinity purified), 1:1,000; anti-synuclein syn211, 1:1000; anti-SUMO2, 1:500. All secondary antibodies coupled to HRP were used in dilution 1:4000. For developing the membrane equal volumes of ECL-1 and ECL-2 reagents were mixed, applied for 1 
min onto the membrane. The enzymatic activity of the peroxidase coupled to the secondary antibody leads to light reaction of luminal (Component of the ECL solution). For detection of the chemiluminiscence autoradiography films (Hyperfilm ${ }^{\mathrm{TM}} \mathrm{ECL}^{\mathrm{TM}}$, Amersham Biosciences) were used. The autoradiography films were exposed for different time periods to the membrane in an autoradiography cassette (Hypercassette ${ }^{\mathrm{TM}}$, Amersham Pharmacia Biotech). Finally, the film was developed in a Curix 60 Developer (Agfa, Köln, Germany).

\subsubsection{Methanol-Chloroform protein precipitation}

For precipitating proteins from solution, including detergent solvents, a modified version of the method described from Wessel and Flügge was used (Wessel and Flugge, 1984). To $200 \mu \mathrm{l}$ of protein solution $0.6 \mathrm{ml}$ of methanol and 0.2 $\mathrm{ml}$ of chloroform were added. Sample was vortexed and $0.6 \mathrm{ml}$ of water was added. The mixture was spun at $9,300 \times \mathrm{g}$ for $5 \mathrm{~min}$. The upper phase was removed and $0.6 \mathrm{ml}$ methanol were added to the lower phase and the interphase with precipitated protein, mixed and centrifuged at $16,100 \times \mathrm{g}$ for $5 \mathrm{~min}$ to pellet the protein. The supernatant was removed, the pellet was air dried and resuspended in SDS sample buffer.

\subsubsection{Colloidal Coomassie Staining}

For in gel staining of proteins seprated by SDS-PAGE Colloidal Coomassie Staining method (Neuhoff et al., 1988) was applied, which allows high sensitivity and clear background using. A polyacrylamide gel was fixed in $40 \%(\mathrm{v} / \mathrm{v})$ ethanol, $10 \%(\mathrm{v} / \mathrm{v})$ acetic acid for at least $60 \mathrm{~min}$. The gel was washed with distilled water two times for 10 min. Four parts Dye stock solutions were mixed with one part 100\% methanol to obtain Dye working solution. The gel was stained overnight and subsequently destained with $1 \%(\mathrm{v} / \mathrm{v})$ acetic acid.

\subsubsection{In vitro aggregation of $\alpha$-synuclein}

Experimental procedures described in this section were performed by Pinar Karpinar and Dr. Markus Zweckstetter (NMR-based Structural Biology, Max-Planck Institute for Biophysical Chemistry). 


\subsubsection{Preparation of $\alpha$-synuclein fibrils}

Recombinant human wild type, mutant, and post-translationally modified $\alpha$ synuclein solutions were dialysed against $50 \mathrm{mM}$ HEPES buffer with $100 \mathrm{mM} \mathrm{NaCl}$ at $\mathrm{pH}$ 7.4. To remove any potential seeding $\alpha$-synuclein species samples were filtered through $100 \mathrm{kDa}$ molecular weight cut-off (MWCO) filters at $4^{\circ} \mathrm{C}$ prior to aggregation. Protein concentration was adjusted to $1 \mathrm{mg} / \mathrm{ml} .0 .01 \%$ sterile filtered $\mathrm{NaN}_{3}$ was included in the aggregation mixtures, which were then incubated in glass vials at $37^{\circ} \mathrm{C}$ with constant stirring at $200 \mathrm{rpm}$. For every experiment, duplicates were prepared.

\subsubsection{Thioflavin T (ThioT) Fluorescence Measurements}

Aliquots $(5 \mu \mathrm{l})$ were withdrawn from $\alpha$-synuclein aggregation samples and added to $2 \mathrm{ml}$ of $5 \mu \mathrm{M}$ ThioT in $50 \mathrm{mM}$ Glycine- $\mathrm{NaOH} \mathrm{pH}$ 8.2. Fluorescence measurements were carried out on Cary Eclipse Spectrofluorometer (Varian) using $3.5 \mathrm{ml}$ quartz cuvettes (Hellma, Germany) with a path length of $1 \mathrm{~cm}$. Fluorescence emission spectra were recorded from 465 to $600 \mathrm{~nm}$, using excitation wavelength of $446 \mathrm{~nm}$, an integration time of 0.1 second, and both excitation and emission bandwidths of $10 \mathrm{~nm}$. Kinetic aggregation traces were generated from time traces of Thioflavin T (ThioT) fluorescence intensity at $482 \mathrm{~nm}$ and corrected for free ThioT fluorescence. Data was represented as mean \pm standard deviation, $n=2$.

\subsubsection{Transmission Electron Microscopy (TEM)}

For negative staining, a protein-containing solution was applied to glowdischarged carbon coated grids and stained with 1\% uranyl acetate. Images were taken in a Philips CM120 electron microscope (Philips Inc.) at a defocus of $2.3 \mu \mathrm{m}$ using a TemCam 224A slow scan CCD camera (TVIPS, Gauting, Germany).

\subsubsection{Animal procedures}

All animal experiments were carried out according to the regulations of the local animal research council, legislation of the State of Lower Saxony (Braunschweig) and the European Community Council Directive of $24^{\text {th }}$ November 1986 (86/609/EEC). Adult female Wistar rats (University Hospital Animal Facility, Göttingen) of $250-280 \mathrm{~g}$ of weight were housed at $12 \mathrm{~h} / 12 \mathrm{~h}$ of light/dark cycle, provided with food and water ad libitum. Animals were deeply anaesthetised by $7 \%$ 
chloral hydrate intraperitoneally before the surgery. All animals were sacrificed by $\mathrm{CO}_{2}$ inhalation.

\subsubsection{Stereotaxic injection into the rat brain}

After a $7 \%$ chloral hydrate intraperitoneally anaesthesia the head of the rat was fixed by ear bars and a jaw holder in a stereotaxic frame ("Kopf" Instruments). The skin covering the skull was longitudinally cut from the imaginary line connecting the eyes in the front till the imaginary line connecting the auditory channels in the back. Remaining connective tissue was carefully removed aside by a scalpel in order to achieve an appropriate visualisation of the skull sutures and visualize the bregma under the operating microscope. Bregma is the crossing point of parasaggital with coronal sutures on the surface of the skull and was defined as "zero" or "start" point. After achieving flat positioning of the skull, the coordinates for the injection were calculated relatively to bregma. For the injection into the left hemisphere SNpc, the following coordinates were used: antero-posterior (AP): 0.53; medio-lateral (ML): +0.22 ; dorso-ventral (DV): -0.77 accordingly with the rat brain atlas (Paxinos and Watson 1986). Glass capillaries filled with mineral oil and the solution for the injection were attached to the Nanoliter2000 injector. The injector was connected to a microprocessor-based controller, Micro4 smart controller which allows for setting the operating parameters, such as the volume and the speed ( $\mathrm{nl} / \mathrm{min}$ ) of the injection. AAV-vectors in PBS in a volume of $2 \mu \mathrm{l}$ were injected into SNpc at a speed of $0,5 \mu \mathrm{l} / \mathrm{min}$.

\subsubsection{Transcardial perfusion and brain tissue processing}

Transcardial perfusion under terminal anaesthesia is a commonly used method for tissue fixation in immunohistochemical protocols. This method takes advantage of the animal's circulatory system to deliver the fixative solution evenly throughout the body tissues, with optimal penetration of the brain. Fixation ensures preservation of the tissue ultra-structure, stabilizes protein and peptide conformation so that antibodies can bind to antigen sites. We used $4 \%$ paraformaldehyde (PFA) in phosphate buffer solution (PBS, $\mathrm{pH}$ 7.4) as a fixative. After a deep anaesthesia with $7 \%$ chloral hydrate $(800 \mathrm{mg} / \mathrm{ml})$ or $\mathrm{CO}_{2}$ inhalation the rat was fixed on rack, the abdominal cavity was opened, and the diaphragm was cut to provide access to the thorax. The ribs were bilaterally cut and the rib cage was open to allow heart 
access. While holding the heart steady, the left ventricle was cut and a blunt-ended needle connected to a catheter was inserted through the ventricle and atrium into the aorta. The needle position was secured in position by clamping. The liver was cut to allow a large part of the blood volume to leave the body and the nose was cut as well to control the procedure efficiency and progress. After approximately $50 \mathrm{ml}$ perfusion with ice-cold PBS, the abdominal aorta was clamped to restrict perfusion to the upper part of the body. Thus, if fluid was flowing freely from the cut nose the needle was considered to be appropriately positioned. The pump was operated with a speed of $15-20 \mathrm{ml} / \mathrm{min}$ with ice-cold PBS. When the blood has been cleared from the body (ca. $200 \mathrm{ml}$ of PBS), the PBS was replaced with ice-cold 4\% PFA solution $(200 \mathrm{ml})$. The rat was decapitated and the brain carefully removed from the skull and placed into $4 \%$ PFA overnight at $4^{\circ} \mathrm{C}$ for post-fixation. The brain was cryoprotected by dehydration in 30\% sucrose solution in PBS usually for 3 days and then frozen at $-80^{\circ} \mathrm{C}$ until further processing.

\subsubsection{Indirect immunofluorescence on brain slices}

The rat brains were embedded in cryomatrix, and coronary sections $(30 \mu \mathrm{m}$ thickness) of the SNpc region were prepared using a Leica cryostat (Leica CM 3050 S). The brain sections were transferred in 24 well plates filled with PBS supplemented with $0.02 \%$ Sodium azide and kept free floating for the entire staining procedure. The sections were shortly washed with PBS-T $(0.1 \%$ Triton X-100 in PBS) and incubated in a blocking solution for $1 \mathrm{~h}$ at room temperature to avoid unspecific binding of the antibodies. Incubation with primary antibody (anti-alpha synuclein, 1:1000; anti-VMAT2, 1:3000) was performed overnight at $4^{\circ} \mathrm{C}$ in incubation solution for IHC (2.1.6.3). After washing $3 \times 5$ min with PBS, the secondary cy 2 and cy 3 coupled antibodies (1:250 in secondary antibody incubation solutio) were applied for $1 \mathrm{~h}$ at room temperature. The unbound secondary antibodies were washed out with PBS-T ( $3 \times 5 \mathrm{~min})$. The sections were mounted on SuperFrost Plus Microscope Slides and embedded in Polyvinyl alcohol mounting medium with DABCO, coated by coverslips and kept at $4^{\circ} \mathrm{C}$ until microscopy. IHC buffers are listed in 2.1.6.3. 


\subsubsection{Quantification of VMAT-positive neurons in SNpc}

Every third section of the brainstem region containing SNpc was immunostained and quantified. The number of VMAT-positive neurons in the SNpc was assessed using stereological methodology. VMAT-positive neurons in the $\mathrm{SNpc}$ were counted from the left side of each immunostained section from minimum six animals per group by using the optical dissector technique (Stereo Investigator 6.0, MicroBrightField Inc.; Zeiss microscope, Jena, Germany). Counts were performed manually and blinded for treatment. 


\section{Results}

\subsection{In vitro SUMOylation of $\alpha-s y n u c l e i n$}

SUMOylation can be reconstituted in vitro for many targets by using recombinantly expressed SUMO enzymes. This assay offers the advantage that substrate competition for the same enzyme or SUMO-specific proteases, present in eukaryotic lysates can be avoided.

Non-tagged a-synuclein was incubated in the presence of SUMO1 or SUMO2, E1-activating enzyme Aos1/Uba2 (SAE1/SAE2), E2-conjugase Ubc9 and ATP. Some targets (e.g. RanGAP1) can be efficiently modified with just Aos1/Uba2 and Ubc9, however other require E3 ligases for efficient modification. Here I tested

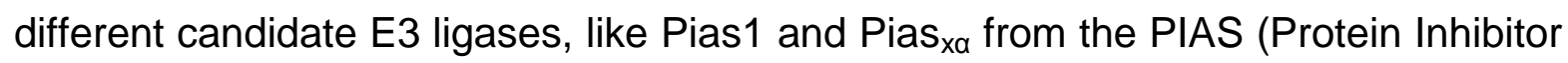
of Activated STAT) family of SUMO E3-like factors, the $33 \mathrm{kDa}$ catalytic domain $(\mathrm{Bp} 2 \Delta \mathrm{FG})$ and the 79-residue fragment IR1+M (Internal Repeat1 + middle domain) of the nucleoporin RanBP2/Nup358 (Pichler et al., 2002), for their ability to facilitate in vitro SUMOylation of a-synuclein. As shown in Figure 3-1, Aos1/Uba2 and Ubc9 were sufficient to conjugate SUMO1 to a-synuclein, which resulted in an approximately $20 \mathrm{kDa}$ shifted band that could be detected by an $\alpha$-synuclein specific antibody. Without energy source present (in the absence of ATP) no shifted band could be detected, which confirmed that the $\approx 35 \mathrm{kDa} \alpha$-synuclein positive band is a result of SUMO1 covalent modification. Among the E3 ligases tested, Pias ${ }_{x \alpha}$ was able to slightly enhance the efficiency of synuclein SUMOylation compared to the sample without E3. The reaction condition resulting in significantly higher yield of SUMOylated $\alpha$-synuclein was the one containing $I R 1+M$. The 79 residue $I R 1+M$ fragment was shown to have full activity but reduced target specificity, which could explain the higher SUMOylation efficiency (Pichler et al., 2004). 


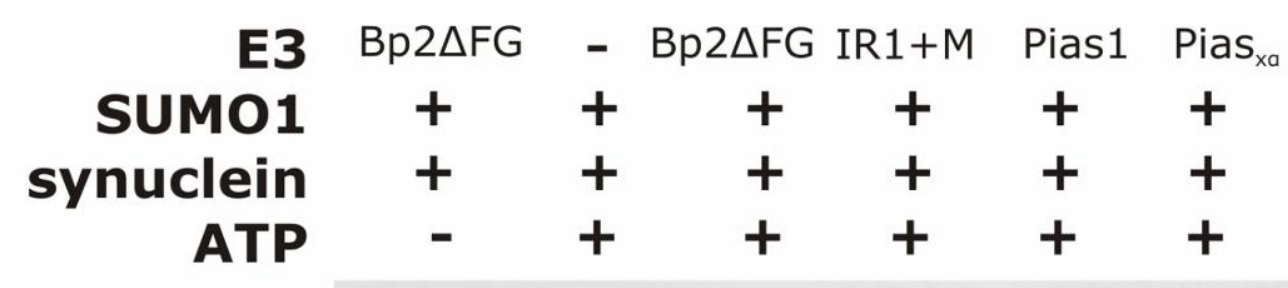

\section{$37 \mathrm{kDa}$}

\section{$15 \mathrm{kDa}$}

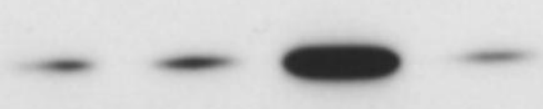

Figure 3-1. In vitro SUMOylation of $\alpha$-synuclein: $500 \mathrm{ng} \alpha$-synuclein, $500 \mathrm{ng}$ SUMO1, 150 ng Aos1/Uba2, 200 ng Ubc9 and 5-10 ng E3 ligase were incubated for $30 \mathrm{~min}$ at $30^{\circ} \mathrm{C}$ with and without ATP. Reactions were stopped by addition of SDS sample buffer. Different reaction samples were analysed by SDS-PAGE and immunoblotting with mouse monoclonal anti- $\alpha$-synuclein antibody (Syn211, Zymed).

The same in vitro SUMOylation experiment was performed using recombinant SUMO2 isoform. As shown in Figure 3-2 a-synuclein was modified also by SUMO2. In contrast to SUMO1, SUMO2 could not be conjugated to alpha synuclein in the absence of E3 ligase. From the E3 ligases tested, efficient SUMO2 modification was achieved by RanBP2 $\triangle \mathrm{FG}$ and Pias $_{\mathrm{xa}}$. In the reaction condition containing Pias1, a 35kDa $\alpha$-synuclein positive band was not detected, indicating that Pias1 failed to facilitate synuclein SUMO2 modification. In vitro SUMO2 conjugation to $\alpha$ synuclein was more efficient than SUMO 1.

For both SUMO1 and SUMO2 isoforms complementary experiments (e.g. silencing of potential E3s) need to be done to disclose the specific E3 ligase targeting a-synuclein for SUMOylation in vivo.

a-Synuclein was recombinantly expressed and purified as described in Methods section 2.2.7.1. SUMO1 and SUMO2 isoforms as well as E1, E2 and different E3-enzymes were kindly provided by Prof. F. Melchior's lab. 


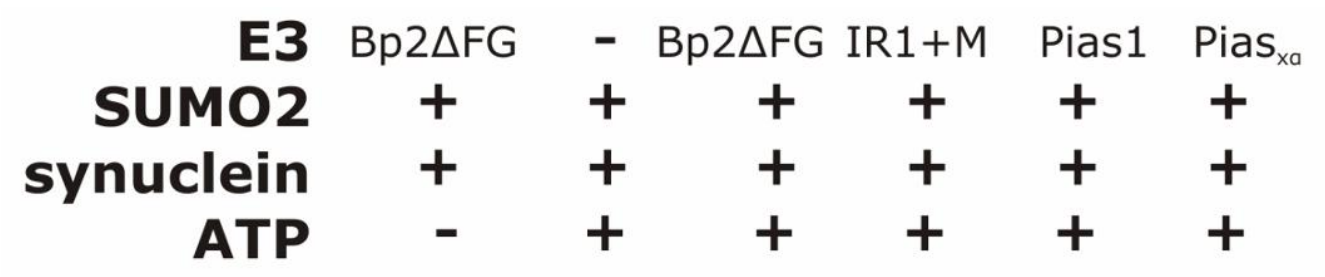

\section{$37 k D a$}

\section{$15 \mathrm{kDa}$}

Figure 3-2. In vitro conjugation of SUMO2 to a-synuclein: $500 \mathrm{ng}$ a-synuclein, 500 ng SUMO2, 150 ng Aos1/Uba2, 200 ng Ubc9 and 5-10 ng E3 ligase were incubated for $30 \mathrm{~min}$ at $30^{\circ} \mathrm{C}$ with and without ATP. Reactions were stopped by addition of SDS sample buffer. After SDS-PAGE different samples were immunoblotted with mouse monoclonal anti- $\alpha$-synuclein antibody (Syn211, Zymed).

\subsection{SUMOylation of $\alpha$-synuclein in HeLa cells}

To confirm that a-synuclein is a SUMOylation target, HeLa cells cotransfected with plasmids encoding $\mathrm{His}_{6}$-tagged SUMO isoforms and a-synuclein were used. Cells were calcium-phosphate transfected either with His 6 -SUMO1 (Figure 3-3, A) or $\mathrm{His}_{6}$-SUMO2 (Figure 3-3, B) and a-synuclein. Control samples were complemented with empty vector. Cells were lysed under denaturing conditions and total His-tagged SUMO substrates were isolated by $\mathrm{Ni}$-affinity chromatography. Only in the presence of SUMO and $\alpha$-synuclein, higher molecular weight species positive for $\alpha$-synuclein could be detected in the eluate of His-tagged proteins, which indicates a specific covalent SUMO conjugation to a-synuclein. As opposed to Dorval V. et al. (2006) we could show that $\alpha$-synuclein can be modified equally well by both SUMO1 and SUMO2 and not preferentially by SUMO1. 
A

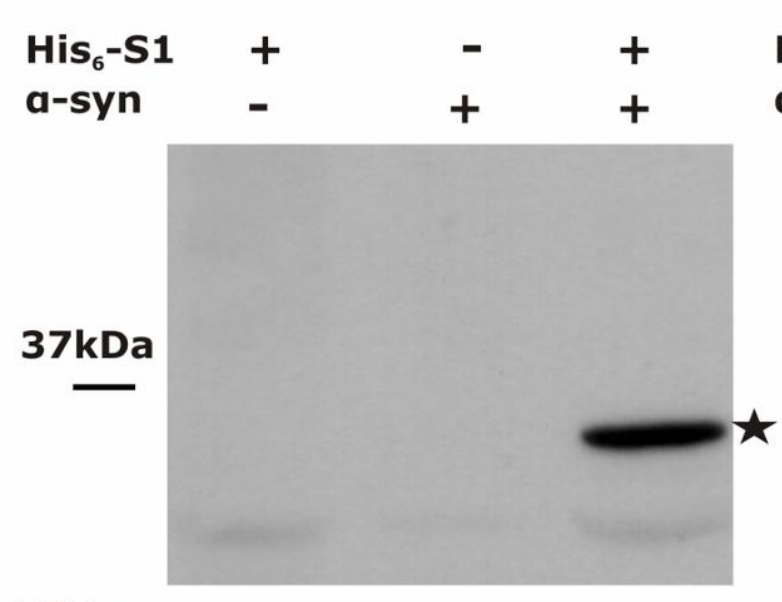

\section{$15 \mathrm{kDa}$}

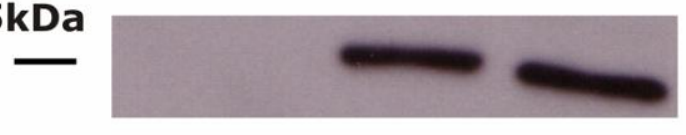

WB: anti-a-synuclein
B

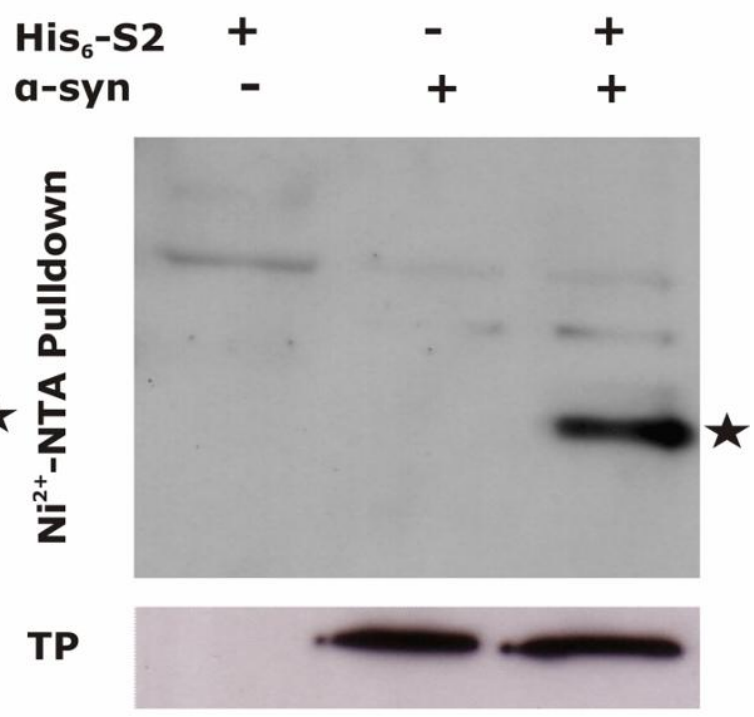

WB: anti-a-synuclein

Figure 3-3. a-Synuclein is modified by SUMO in HeLa cells. Plasmids encoding

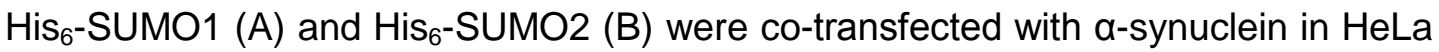
cells. Control samples were complemented with empty vector. SUMO substrates were purified by $\mathrm{Ni}^{2+}$-affinity chromatography and a SUMOylated $\alpha$-synuclein band at $\approx 35 \mathrm{kDa}$ (marked with an asterisk) was detected with $\alpha$-synuclein specific antibody. Total levels of $\alpha$-synuclein expression are shown in the bottom panel (TP).

The $\alpha$-synuclein immunoreactive species were detected at $\approx 35 \mathrm{kDa}$ for both SUMO1 and SUMO2, which corresponds to the SDS-PAGE mobility shift caused by conjugation of a single molecule SUMO to $\alpha$-synuclein. Even though SUMO 2 can form polymeric chains in vitro and has been shown to be conjugated in a form of multimeric chains to substrates like PML in vivo (Tatham et al., 2001), only a single molecule His-tagged SUMO 2 was found to be conjugated to a-synuclein in HeLa cell lysates.

\subsection{Effects of familial PD mutations on $\alpha$-synuclein SUMOylation}

Pathogenic mutations of $\alpha$-synuclein A30P and A53T have been identified in rare familial cases of PD (Polymeropoulos et al., 1997; Kruger et al., 1998). I thus examined whether A30P and A53T mutations would affect on $\alpha$-synuclein SUMOylation (Figure 3-4). HEK 293 cells were cotransfected with $\mathrm{His}_{6}-\mathrm{SUMO} 2$ and wild type or mutant forms of $\alpha$-synuclein. SUMOylated proteins were $\mathrm{Ni}^{2+}-\mathrm{NTA}$ precipitated and probed with $\alpha$-synuclein antibody. As shown in Figure 3-4, A30P and A53T Parkinson's disease mutants had no effect on SUMOylation. 


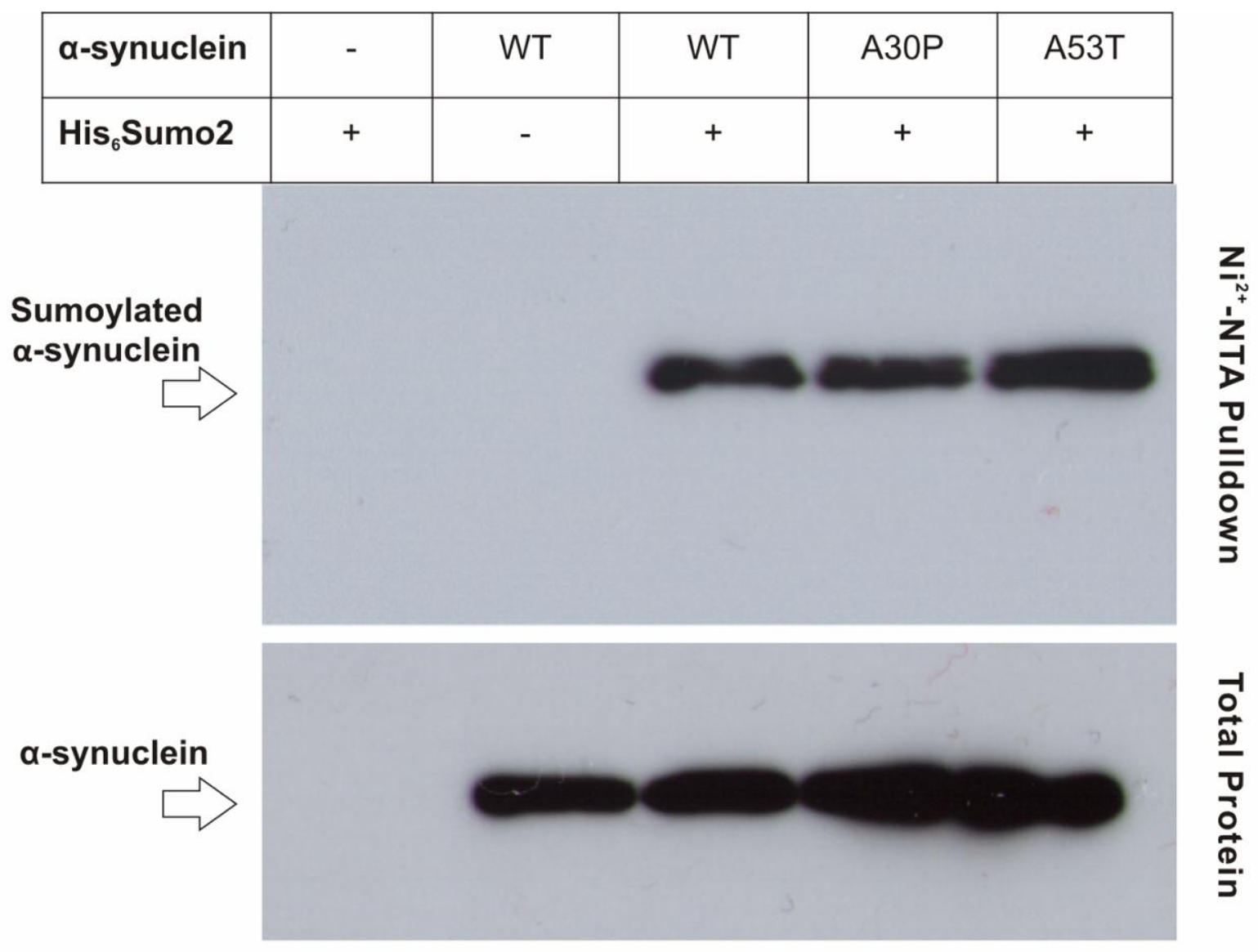

WB: anti-myc 9E10

Figure 3-4. Familial Parkinson's disease related mutants $\mathrm{A} 30 \mathrm{P}$ and $\mathrm{A} 53 \mathrm{~T}$ are SUMOylated. Plasmids encoding $\mathrm{His}_{6}-\mathrm{SUMO} 2$ and $\alpha$-synuclein WT, A30P or A53T were co-transfected in HEK cells. SUMO substrates were purified by nickel-affinity chromatography and SUMOylated $\alpha$-synuclein was detected in all three conditions expressing wt or mutant $\alpha$-synuclein and $\mathrm{His}_{6}-\mathrm{SUMO} 2$. Total levels of $\alpha$-synuclein expression are shown in the bottom panel (Total Protein).

\subsection{FRET-FLIM analysis of $\alpha$-synuclein SUMOylation in the CSM14.1 cell line}

We employed Förster Resonance Energy Transfer (FRET) measured by Fluorescence Lifetime Imaging Microscopy (FLIM) as a complementary method to confirm the sumoylation of $\alpha$-synuclein in morphologically intact neuronal cells. FRET is based on energy transfer from an excited state donor chromophore to a spectrally suitable acceptor chromophore within nanometer range of the former (Forster, 1948). As in FLIM-FRET the fluorescence lifetime of the donor is measured and used to interpret the close proximity problems it is independent of the concentration and spectral problems typically seen with intensity based FRET methods. For example, the fluorescence lifetime is an intrinsic property of an 
excited fluorochrome independent of excitation intensity and fluorophore concentration; additionally, in combination with 2-photon excitation sources FRETFLIM provides high spatial resolution. Because of the mentioned advantages we used FRET-FLIM to investigate $\alpha$-synuclein/SUMO interactions in neuronal cells. CSM14.1 neuronal cells express the dopaminergic markers Nurr1, tyrosine hydroxylase and ALDH2 and can therefore be characterized as a dopaminergic cell line (Haas and Wree, 2002). CSM cells were transfected with a-synuclein-mCFP (monomeric Cyan Fluorescent Protein) fused to the C-terminus of $\alpha$-synuclein and SUMO1 fused to monomeric Venus using Lipofectamine (Zacharias et al., 2002; Pommereit and Wouters, 2007). Two different SUMO constructs were used: one driving the expression of mature SUMO with revealed C-terminal Gly-Gly motif (mVenus-SUMO1- $\Delta \mathrm{C} 4$ ) and a second one as a negative control for SUMO conjugation, a C-terminal deletion mutant that cannot be conjugated (mVenusSUMO1- $\triangle \mathrm{C} 7$ ). 36 hours after transfection cells were fixed and permeabilized.

Comparison of the cumulative histograms of the fluorescence lifetime distribution derived from several $\alpha$-synuclein-CFP expressing cells to those, obtained from cells transfected with $\alpha$-synuclein-mCFP alone and in combination with $\mathrm{mVenus} / \mathrm{mVenus-SUMO}-\Delta 4 / \mathrm{mVenus-SUMO}-\Delta 7$, revealed a significant reduction in the CFP lifetime in the mVenus-SUMO- $\Delta 4$ and mVenus-SUMO- $\Delta 7$ coexpressing cells. Figure $3-5 \mathrm{c}$ shows $37.18 \%$ (S.E.M 3.84) probability for FRET to occur in samples transfected with $\alpha$-synuclein-mCFP and mVenus-SUMO- $\Delta 4$ and $10.97 \%$ (S.E.M. 1.42) for cells cotransfected with $\alpha$-synuclein-mCFP and mVenusSUMO- $\Delta 7$. The control condition lacking SUMO1, $\alpha$-synuclein-mCFP and mVenus cotransfection, showed $0.53 \%$ (S.E.M. 0.28) FRET. The higher FRET efficiencies in cells expressing mature SUMO1 compared to non-conjugatable SUMO1 implies that it is predominantly the covalent attachment of SUMO1 to $\alpha$-synuclein that accounts for the fluorescence lifetime decrease. The $\approx 11 \%$ probability for FRET in the condition of $\alpha$-synuclein-mCFP and mVenus-SUMO- $\Delta 7$ coexpression raises the possibility of non-covalent interaction between $\alpha$-synuclein and SUMO. Such an interaction failed to be confirmed in a biochemical SUMO binding assay (not shown). This might be due to the very low affinity of a potential $\alpha$-synuclein - SUMO interaction.

The FRET intensity distribution shows that a-synuclein - SUMO1 interaction/conjugation is not confined to certain cellular compartment. 

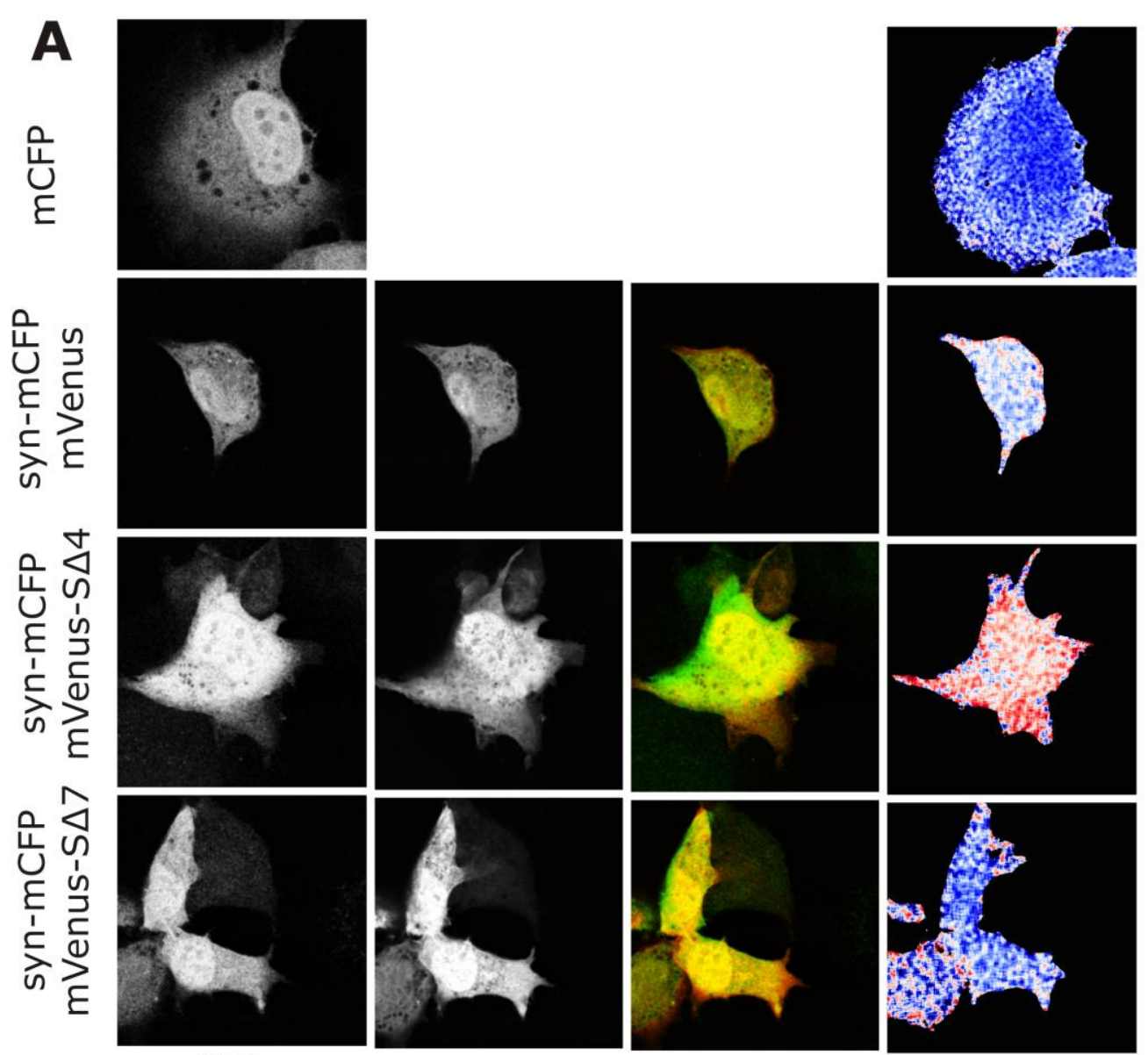

mCFP

mVenus

RGB Overlay

FRET efficiency

B
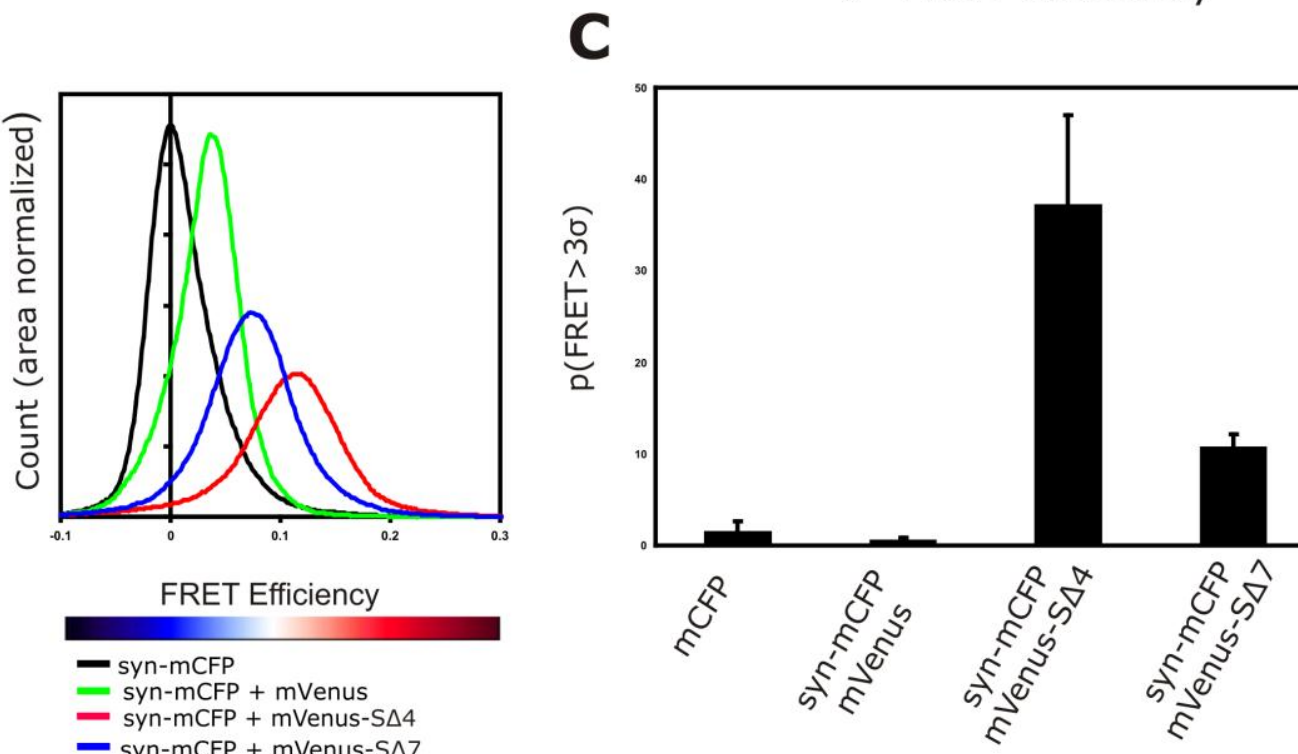

Figure 3-5. SUMOylation of $\alpha$-synuclein detected by FRET/FLIM in intact single cells. FRET/FLIM measurements in CSM14.1 cells transfected with the energy donor a-synuclein-mCFP only, and together with mature SUMO1 (mVenus-S $\Delta 4$ ) or non-conjugatable SUMO1 (mVenus-S $\Delta 7$ ) fused to monomeric Venus as energy acceptor. (A) shows representative distribution of the donor and acceptor constructs (left two columns; merged in third column) and representative fluorescence lifetime images (right panels). The blue colour represents the life time of the donor (mCFP), while the red gamma represents reduction of MCFP life time and presence of FRET. 
The cumulative histograms of FRET efficiencies are shown in (B), and panel (C) provides the respective quantitative analysis of the histograms. The mean lifetime of the donor only was determined from the summarized donor histograms and used as $0 \%$ FRET point. A 3 -sigma value (=98.5\% statistical confidence) was determined from the donor histogram. The number of pixels on a donor-acceptor image with fluorescence lifetime lower than 3-sigma cut off is indicated as fraction from the total number of pixels. Data sets are presented as averages ${ }_{-}^{+}$SEM. mCFP $(1.46 \% \pm 1.20) ; \quad$ syn-mCFP+mVenus $\quad(0.53 \% \pm 0.28) ; \quad$ syn-mCFP+mVenus-S $\Delta 4$ $(37.18 \% \pm 3.84)$; syn-mCFP+ mVenus-S $\Delta 7(10.97 \% \pm 1.42)$.

The quantification of the FRET/FLIM experiments presented in this section was performed by Dr. Mika Ruonala (European Neuroscience Institute, Göttingen; Currently: Center for Membrane Proteomics, University of Frankfurt).

\section{5. $\alpha-S y n u c l e i n$ is SUMOylated in brain tissue of $\mathrm{His}_{6}$-SUMO2 transgenic mice}

In order to test whether a-synuclein is a SUMO target in vivo, we used $\mathrm{His}_{6}{ }^{-}$ SUMO2 transgenic mice. The SUMO2 transgenic mice used in the following experiment were generated by Dr. Marilyn Tirard (Max Planck Institute for Experimental Medicine, Prof. Nils Brose Lab). The integration of the transgene was verified by PCR-genotyping. Western blot analysis of brain homogenate confirmed the mild overexpression of the transgene, driven by the neuron-specific Thy 1.2 promoter (Figure 3-6). The transgenic animals overexpress $\mathrm{His}_{6}-\mathrm{SUMO} 2$ in the CNS which allows enrichment of neuronal SUMO targets under denaturing conditions using $\mathrm{Ni}^{2+}$-NTA affinity chromatography.

Brain tissue from wild type and $\mathrm{His}_{6}-\mathrm{SUMO} 2$ transgenic mice was lysed as described in section 2.2.5.3. Usually two mouse brains ( $\approx 0,5 \mathrm{~g}$ each) were used per sample condition. Brain lysates from wild type and transgenic animals were shown to contain comparable total amounts of a-synuclein (Figure 3-7a). 


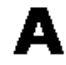

\section{\begin{tabular}{|l|l|l|} 
Thy1.2 promoter & $\mathrm{His}_{6}$ & SUMO cDNA \\
\hline
\end{tabular}}

B

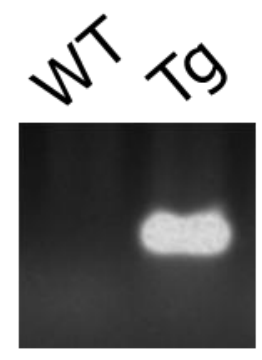

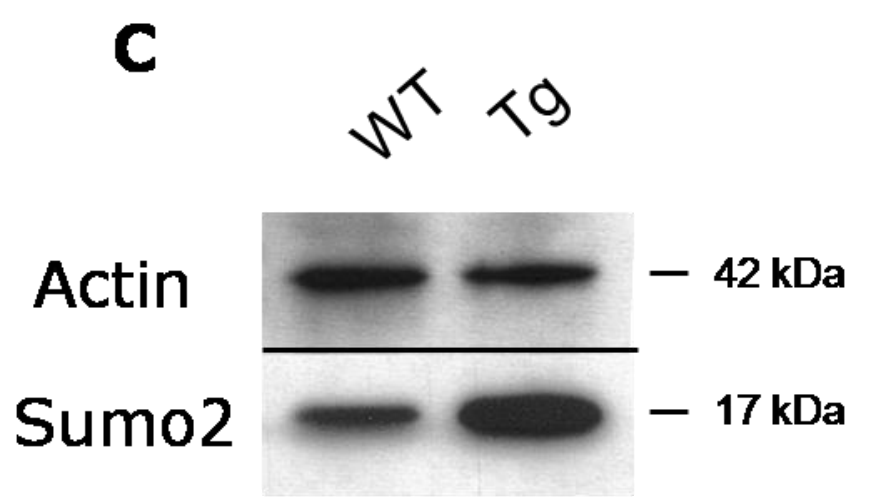

Figure 3-6. Generation of Thy1.2: $\mathrm{His}_{6}-\mathrm{SUMO} 2$ transgenic mice. Human $\mathrm{His}_{6}{ }^{-}$ tagged SUMO2 cDNA was amplified by PCR from a corresponding pET28-hSUMO2 vector and inserted into the pTSC21 vector, which drives transgene expression under the Thy1.2 promoter (A). After linearization, purified DNA was injected into pronuclei of FVB/N embryos. To identify founder mice, genomic DNA was prepared from tail biopsies and analyzed by PCR (B). Positive founders were then bred to generate independent transgenic mouse lines. Western blot analyses revealed overexpression of unconjugated SUMO2 in brain homogenates of positive transgenic animals $(\mathrm{C})$.

Total SUMO2 conjugated proteins were isolated by $\mathrm{Ni}^{2+}$-affinity chromatography (Figure 3-7b) and analysed by Western Blotting with an anti-a-synuclein antibody, recognizing the mouse protein isoform. Only in the elution sample from the $\mathrm{His}_{6}{ }^{-}$ SUMO2 transgenic mice higher molecular weight species positive for a-synuclein were detected. The electrophoretic mobility shift to approximately $35 \mathrm{kDa}$ corresponds to conjugation of single SUMO2 (arrow) and the higher species to two and more SUMO2 molecules (braces). The latter could result from SUMO2 chain formation or from simultaneous multiple mono-SUMO2 conjugation at different lysine acceptor sites. 
A

B

$\underline{\text { Total Brain Lysate }} \underline{\mathrm{Ni}^{2+} \text {-NTA Pulldown }}$

WT Tg

WT Tg

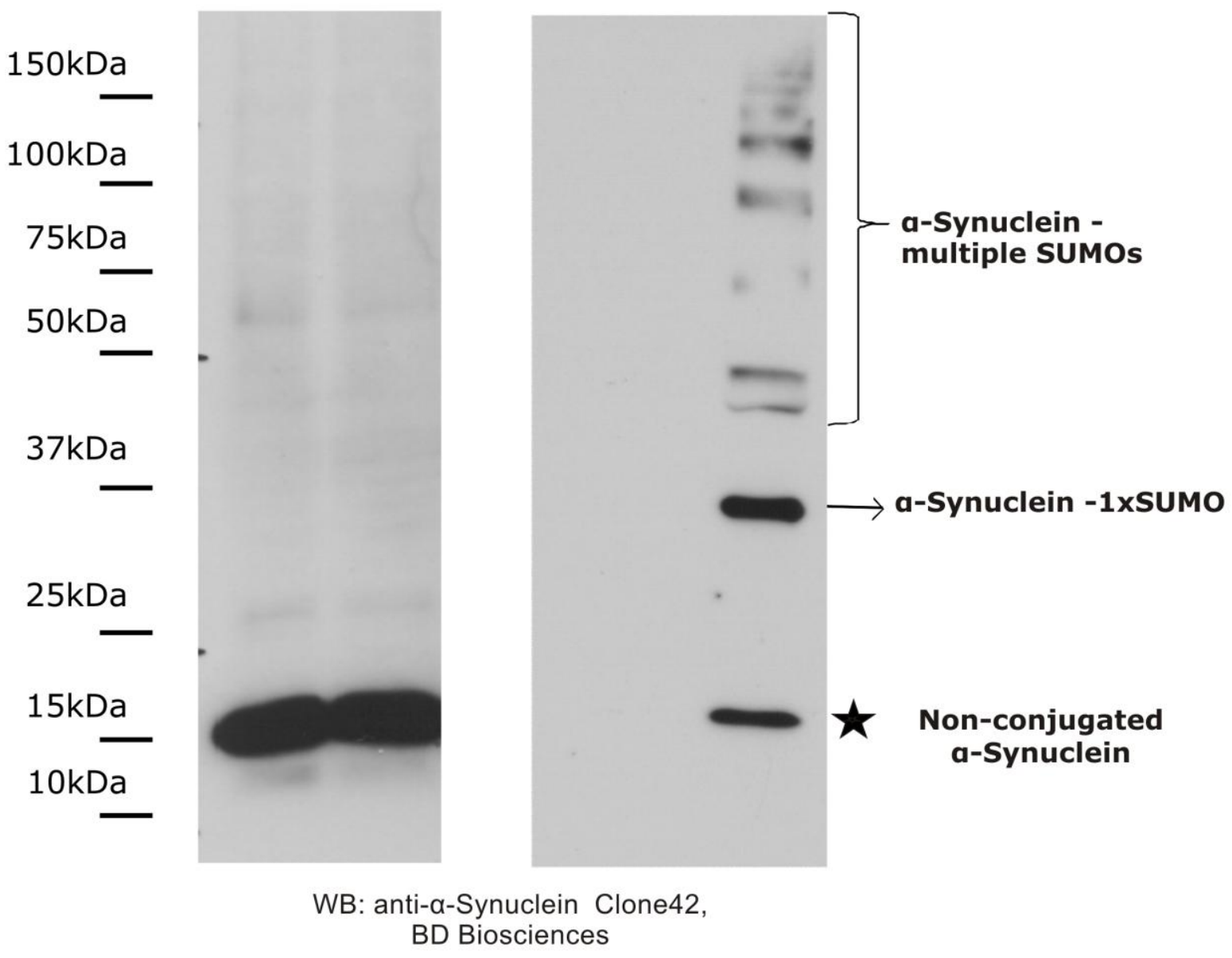

Figure 3-7. In vivo SUMOylation of a-synuclein. Brain material from wild type (WT) and $\mathrm{His}_{6}-\mathrm{SUMO} 2$ transgenic mice $(\mathrm{Tg})$ was lysed and total SUMO2 conjugated proteins were isolated using $\mathrm{Ni}^{2+}$-NTA affinity chromatography. Total brain lysates (A) and eluates (B) were probed with anti- $\alpha$-synuclein antibody specific for mouse $\alpha$ synuclein. Synuclein modified by a single and multiple SUMO2 molecules, indicated with an arrow and braces, respectively, is detected only in the eluate obtained from $\mathrm{His}_{6}$-SUMO2 transgenics. Non-conjugated $\alpha$-synuclein (asterisk) is most probably a result of residual iso-peptidase activity.

SUMO2 conjugation to $\alpha$-synuclein in HeLa or HEK 293 cells resulted in occurrence of a single $35 \mathrm{kDa}$ SUMOylated form in contrast to the higher molecular weight synuclein-SUMO2 conjugates observed in mice brain lysates. Reasons for that could be that the higher molecular weight synuclein-SUMO2 conjugates are more stable in neuronal tissues or that multiple SUMO2 conjugation to $\alpha$-synuclein occurs specifically in neuronal cells. 


\subsection{Identification of $\alpha$-synuclein SUMOylation sites}

\subsubsection{Mutagenesis analysis}

Mapping the SUMO conjugation site for a target protein is of crucial importance, since generating mutants which are deficient in SUMO acceptor sites is a key step in finding the role of SUMOylation in the context of a specific target.

For many substrates, among which are RanGAP1 (Matunis et al., 1996; Mahajan et al., 1997), PML (Kamitani et al., 1998) and Sp100 (Sternsdorf et al., 1999), the SUMO acceptor lysine is part of a consensus sequence $\Psi-K-X-D / E$, where $\Psi$ is a hydrophobic amino acid and $X$ stands for any amino acid. This consensus motif is being recognized by Ubc9 when it is part of an extended loop or when it is present in an unstructured area (Bernier-Villamor et al., 2002; Macauley et al., 2006). However, SUMO conjugation sites have been identified which are not parts of consensus motifs (e.g.Mdm2, Daxx, CREB).

a-Synuclein contains two lysine residues $-\mathrm{K} 96$ and K102, which are part of the V-K-K-D and G-K-N-E motifs which could be potential SUMO conjugation sites. Therefore point mutations were inserted using PCR-directed mutagenesis leading to lysine 96 and 102 substitution with arginine. These mutants were cotransfected with $\mathrm{His}_{6}-\mathrm{SUMO} 2$ in HEK 293 and tested for their SUMOylation levels using $\mathrm{Ni}^{2+}$-NTA pulldown (Figure 3-8, lanes 8 and 9). Compared to wild type, both the K96R and K102R a-synuclein mutants showed a minor reduction in SUMOylation, suggesting that K96 or K102 can principally serve as SUMO conjugation sites. The remaining 13 lysine residues of $\alpha$-synuclein are not part of consensus motifs, but given the fact that $\alpha$-synuclein is a natively unstructured protein, those lysines are most probably accessible and can potentially be used by the SUMOylation machinery as acceptor sites. The following double and triple lysine-arginine mutants were cloned for further analysis of the SUMO-synuclein conjugation:

- Synuclein mR1 - K6R, K10R, K12R (in brief mR1),

- Synuclein mR2 - K21R, K23R (in brief mR2),

- Synuclein mR3 - K32R, K34R (in brief mR3),

- Synuclein mR4 - K43R, K45R (in brief mR4),

- Synuclein mR5 - K58R, K60R (in brief mR5). 
All constructs were myc-tagged to assure that mutations within $\alpha$-synuclein would not interfere with antibody detection.

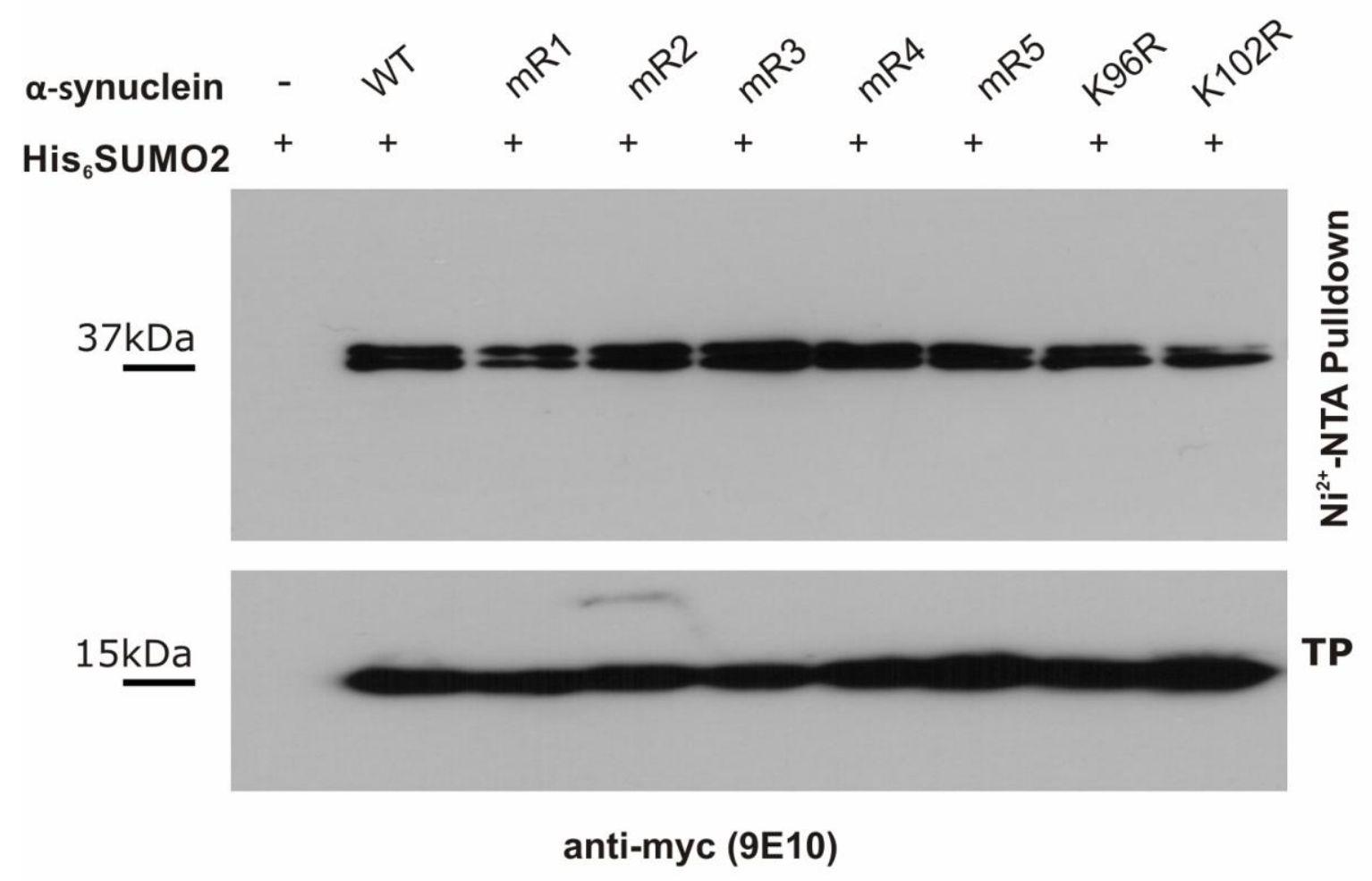

Figure 3-8. Mutagenesis analysis of $\alpha$-synuclein in HEK 293 cells. SUMOylation of wild type $\alpha$-synuclein was compared to different lysine-arginine mutants ( $m R 1, m R 2$, mR3, mR4, mR5, K96R, K102R) for identification of SUMO acceptor site, using $\mathrm{Ni}^{2+}$ NTA pulldown (upper panel). Total protein levels (TP) of wild type $\alpha$-synuclein and respective mutant forms are shown in the lower panel. Elution samples and total lysates were ran on a $4-12 \%$ NuPAGE Bis-Tris gel (Invitrogen) and analysed by Western blot with anti-myc antibody.

$\mathrm{Ni}^{2+}$-pulldown from HEK 293 cells transfected with His ${ }_{6}-\mathrm{SUMO} 2$ and wild type synuclein or one of the above listed mutant constructs failed to reveal a mutation that abolishes SUMO conjugation (Figure 3-8, upper panel lane 2-7). Taken together, the mutagenesis results point out that $\alpha$-synuclein can be SUMOylated at multiple lysine acceptor sites.

The pulldown experiments in HeLa and HEK 293 cells showed that at a steady state level $\alpha$-synuclein is modified by a single SUMO molecule, and the close proximity of $\mathrm{K} 96$ and $\mathrm{K} 102$ suggests that for sterical reasons SUMOylation occurs alternatively at either one of the two sites. Moreover, both consensus site lysines are positioned at the transition between the hydrophobic NAC core and the acidic C-terminal region of $\alpha$-synuclein, which might be of specific functional 
significance. We thus hypothesised that the two lysines might together represent an "alternative SUMOylation site" as the major SUMO acceptor site(s) in a-synuclein.

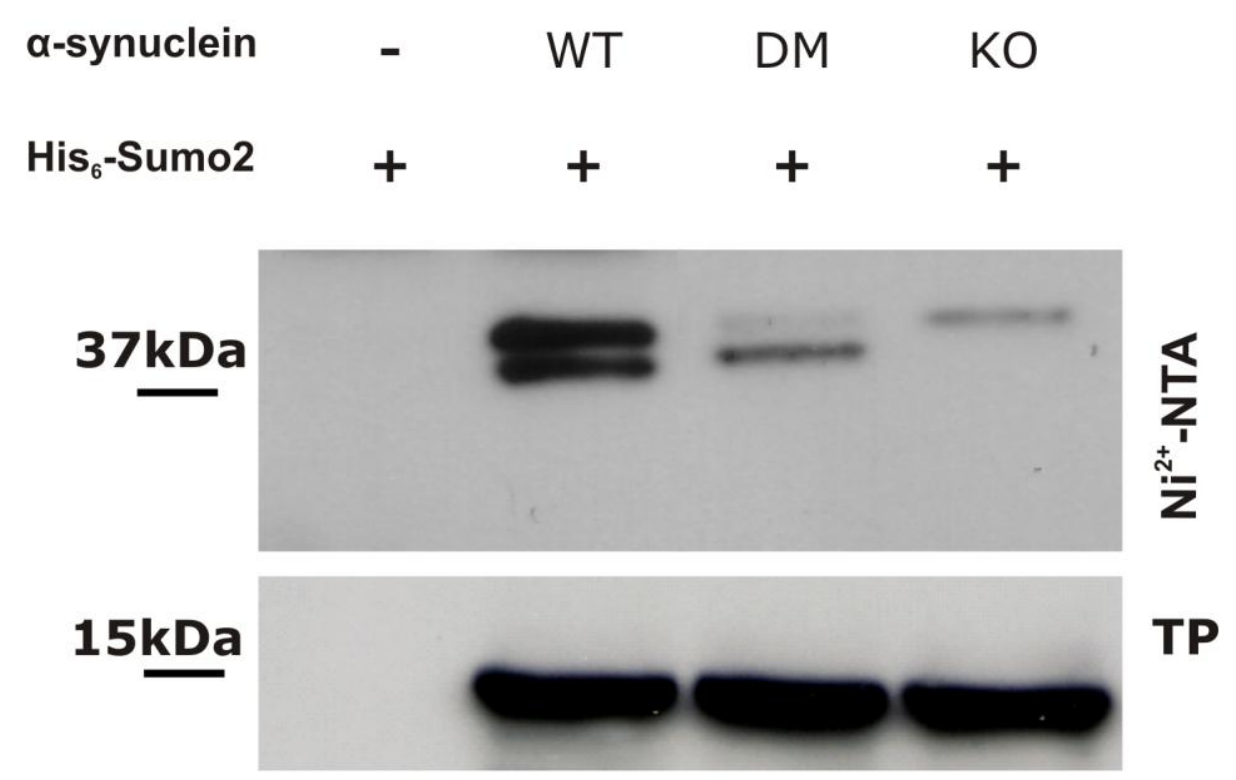

\section{WB: anti-myc 9E10}

Figure 3-9. $\alpha$-Synuclein-DM and $\alpha$-synuclein-KO show reduction in SUMOylation in HEK 293 cells. SUMOylation of wild type a-synuclein was compared to DM (K96R, K102R) and KO (K6R, K10R, K12R, K21R, K23R, K32R, K34R, K43R, $\mathrm{K} 45 \mathrm{R}, \mathrm{K} 58 \mathrm{R}, \mathrm{K} 60 \mathrm{R})$ mutants for identification of synuclein mutant with significantly reduced SUMOylation (upper panel). Total protein levels (TP) of wild type a-synuclein and respective mutant forms are shown in the lower panel. Elution samples and total lysates were ran on a 4-12\% NuPAGE Bis-Tris gel (Invitrogen) and analysed by Western blot.

To examine this hypothesis two additional mutants were generated: $\alpha$ synuclein-DM (double mutant) having both consensus lysines, K96 and K102, mutated to arginines; and $\alpha$-synuclein- $K O$, having the remaining lysine residues K6R, K10R, K12R, K21R, K23R, K32R, K34R, K43R, K45R, K58R, K60R but not K96 and K102 mutated to arginine (Figure 3-9; Figure 3-10). Pulldown experiments showed that SUMOylated wild type a-synuclein runs in SDS-PAGE as two close bands which can be separated if the samples are run on high resolution gels (4$12 \%$ NuPAGE Bis-Tris, Invitrogen). Simultaneous mutations of the two consensus lysines K96 and K102 (DM) resulted in loss of the upper SUMOylated band, while as single amino-acid substitutions $\mathrm{K} 96 \mathrm{R}$ or $\mathrm{K} 102 \mathrm{R}$ led to a slight reduction of the upper band intensity. In contrast, the inverse a-synuclein-KO (K6R, K10R, K12R, K21R, K23R, K32R, K34R, K43R, K45R, K58R and K60R) mutant caused the 
disappearance of the lower band. This result was confirmed using two different cell lines - HEK 293 cells (Figure 3-9) and HeLa cells (Figure 3-10).

$\begin{array}{lcccc}\text { a-synuclein } & \text { WT } & \text { DM } & \text { KO } & - \\ \text { His }_{6} \text {-Sumo2 } & + & + & + & +\end{array}$

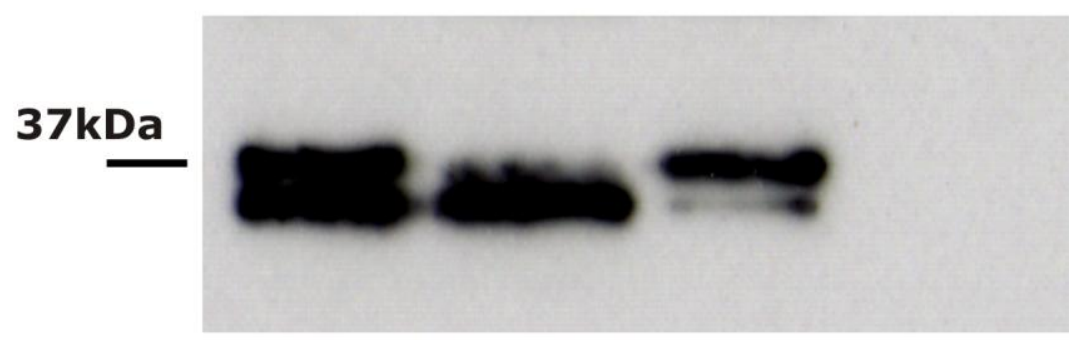

$\mathrm{Ni}^{2+}$-NTA pulldown; WB: anti-myc (9E10)

Figure 3-10. $\alpha$-Synuclein-DM and $\alpha$-synuclein-KO show a reduction in SUMOylation in HeLa cells. Using $\mathrm{Ni}^{2+}$-NTA pulldown SUMOylation of $\alpha$-synuclein-WT was compared to DM (K96R, K102R) and KO (K6R, K10R, K12R, K21R, K23R, K32R, $\mathrm{K} 34 \mathrm{R}, \mathrm{K} 43 \mathrm{R}, \mathrm{K} 45 \mathrm{R}, \mathrm{K} 58 \mathrm{R}, \mathrm{K} 60 \mathrm{R}$ ) mutants for identification of synuclein mutant with significantly reduced SUMOylation. Elution samples and total lysates were ran on SDS-PAGE and analysed by Western blot.

Instead of Lys-Arg substitutions, disruption of the two consensus SUMOylation motifs V- ${ }^{96} \mathrm{~K}-\mathrm{K}-\mathrm{D}$ and G- ${ }^{102} \mathrm{~K}-\mathrm{N}-\mathrm{E}$ can be achieved by mutations in the acidic aspartate and glutamate residues. Alternative mutant to the K96R, K102R variant was generated having both Asp 98 and Glu 104 mutated to alanine (DM-Ala). This mutant showed similar to a-synuclein-DM reduction in SUMO conjugation, thereby confirming $\mathrm{K} 96$ and $\mathrm{K} 102$ as consensus SUMOylation sites (Figure 3-11). 


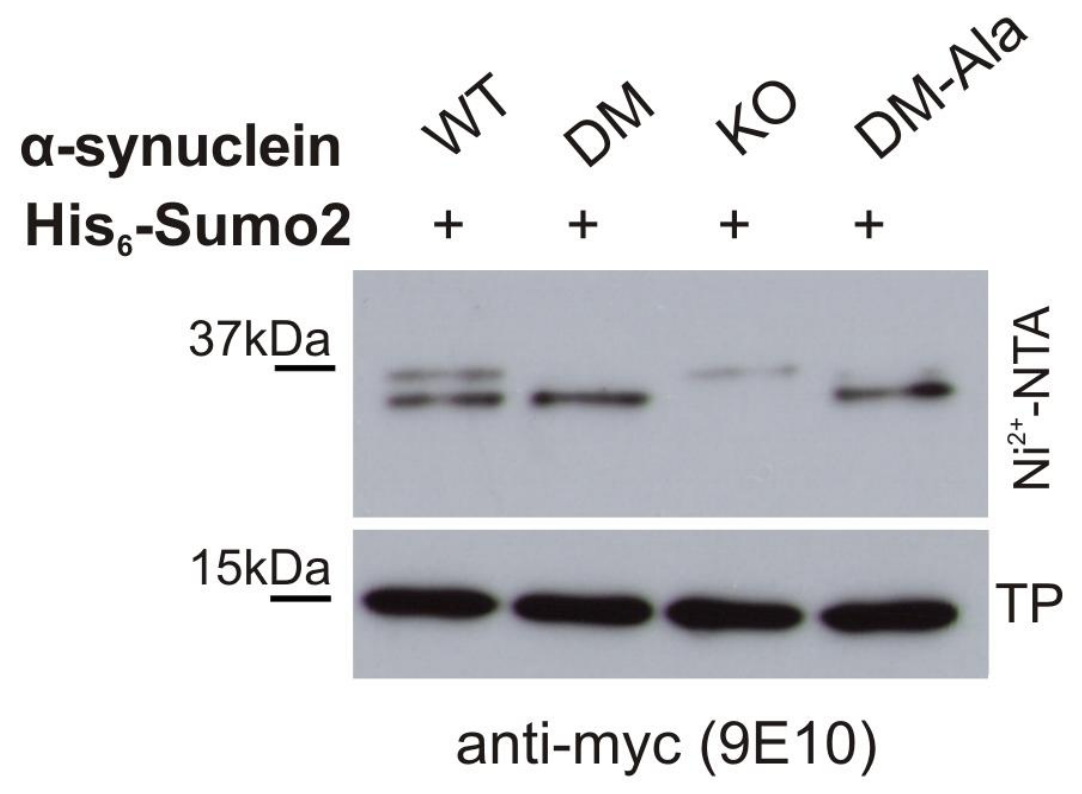

Figure 3-11. Confirmation of a-synuclein consensus SUMOylation sites by mutations of Asp/Glu to Ala. SUMOylation of a-synuclein-WT was compared to DM, DM-Ala and KO mutants (upper panel). a-Synuclein-DM (K96R, K102R) and a-synuclein-DM-Ala (D98A,E104A) show similar reduction in SUMOylation in HEK 293 cells. Total protein levels (TP) of $\alpha$-synuclein-WT and respective mutant forms are shown in the lower panel. Elution samples and total lysates were analysed by Western blot.

These results confirmed our hypothesis that K96 and K102 are alternatively SUMOylated and only the mutant lacking both lysines showed significant reduction in SUMO modification. Moreover $\alpha$-synuclein can be modified at the lysines in the C-terminal acidic region (upper band, K96 or K102) and at the lysine residues in the amphipatic $\mathrm{N}$-terminal region (lower band). The distinct SDS-PAGE mobility of those two species could presumably be due to different structural properties of $\alpha$ synuclein SUMOylated at lysine in the $\mathrm{N}$ - or $\mathrm{C}$-terminal region. Further studies (e.g. CD or NMR) are required to investigate this speculation.

\subsubsection{Overproduction of SUMOylated a-synuclein in E.coli and mass spectrometry}

Lysine-to-arginine substitutions are widely used as SUMO site mapping approach. However, natively unfolded proteins like a-synuclein have most of the potential SUMO acceptor residues in an accessible conformation. In such a case mutating one lysine residue might lead to an 'artificial' site shift and conjugation 
could occur at lysine which is not SUMO modified in the wild type protein. To exclude such false positive results we used mass spectrometric analysis of SUMOylated wild type $\alpha$-synuclein.

\subsubsection{Preparation of a SUMOylated a-synuclein sample for mass spectrometry}

a-Synuclein wild type was cloned into the pET-28a+ vector as described in Methods section 2.2.1.8. E.coli BL21 (DE3) were transformed with pT-E1E2S1 and $\alpha$-synuclein-WT-pET28a+. The polycistronic plasmid pT-E1E2S1 drives the expression of the E1-activating enzyme (Aos1/Uba2), the E2-conjugase ( $\mathrm{Ubc} 9$ ) and SUMO1. a-Synuclein-WT-pET28a+ encodes a-synuclein with C-terminally fused 6xHis tag allowing for $\mathrm{Ni}^{2+}$-NTA affinity purification. In this way the SUMOylation machinery (without E3 ligase) was reconstituted in E.coli and a-synuclein was successfully SUMOylated in the bacterial system without the presence of an E3 ligase. Since the in vitro experiments using recombinant proteins showed that SUMO1, but not SUMO2 can be conjugated to $\alpha$-synuclein in the absence of an E3 ligase, SUMO1 isoform was used for the overproduction of SUMOylated a-synuclein in E.coli.

The crude bacterial lysate was loaded on a $\mathrm{Ni}^{2+}$-NTA affinity column and bound proteins were eluted with high concentrations of imidazole $(250 \mathrm{mM}$ and 500 $\mathrm{mM}$ ). The eluate was further purified using ion-exchange chromatography (Figure 312). Proteins bound to the Mono $Q$ column were eluted by a salt gradient (Buffer $B$ $-1 \mathrm{M} \mathrm{NaCl}, 25 \mathrm{mM}$ Tris $\mathrm{pH}$ 7.7) and fractions were analysed by SDS-PAGE and Coomassie staining. Fractions $12-17$ contained primarily non-modified $\alpha$-synuclein and fractions 19-20 were enriched in SUMO conjugated a-synuclein. Material from those samples was used for further mass spectrometric mapping of a-synuclein SUMOylation sites. 


\section{Anion exchange chromatography}

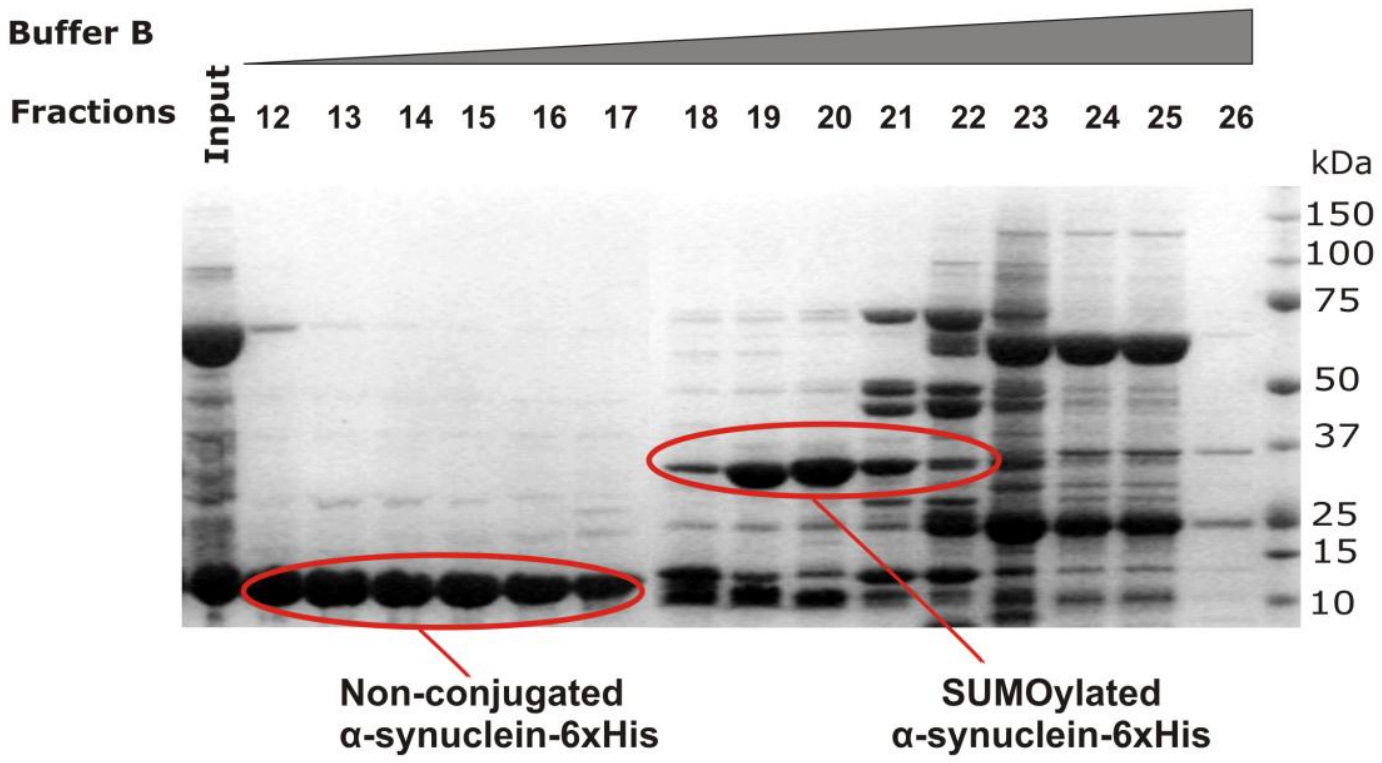

Figure 3-12. Separation of SUMO modified and non-modified $\alpha$-synuclein. The eluate containing His-tagged proteins was separated on Mono $Q$ anion-exchange chromatography and fractions were analysed by SDS-PAGE and Coomassie staining. Fractions 12-17 contained non-modified His-tagged $\alpha$-synuclein and fractions 19-20 were enriched in SUMOylated $\alpha$-synuclein.

\subsubsection{Identification of SUMO conjugation sites by mass spectrometry}

The identification of the SUMO acceptor sites in a-synuclein was done by HeHsuan Hsiao and Dr. Henning Urlaub (Bioanalytical Mass Spectrometry Group, Max-Planck-Institute for Biophysical Chemistry). Therefore only brief summary of the obtained results will be provided here.

a-Synuclein was SUMO1 conjugated in E.coli and purified by affinity chromatography as described in 3.6.2.1. SUMOylated a-synuclein was cut out from 4-12 \% NuPAGE Bis-Tris gel and the protein was digested in-gel with trypsin. LCcoupled ESI-MSMS (Liquid chromatography coupled electrospray tandem mass spectrometry) analysis of the obtained peptides revealed ten lysine residues which were SUMO1 conjugated: K6, K10, K12, K21, K23, K34, K45 and K60 in the Nterminal region, K80 in the hydrophobic NAC region and the consensus K96 in the acidic C-terminus (Figure 3-13). 


\section{a-synuclein}

Amphipatic repeat region

NAC region

Acidic region

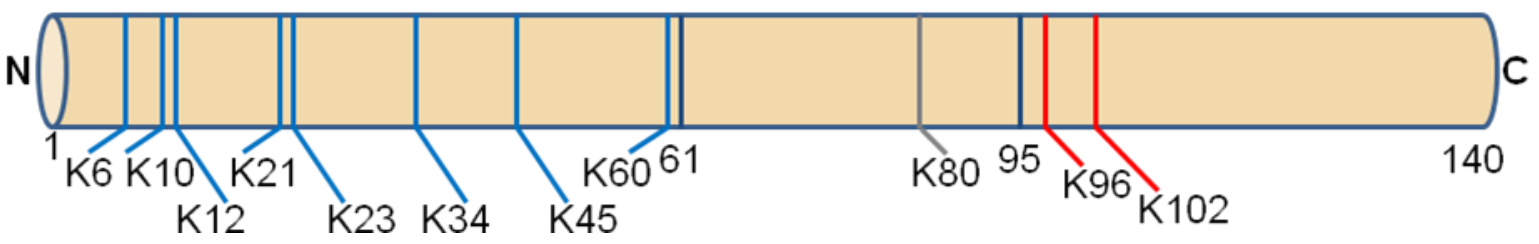

Figure 3-13. Summary of $\alpha$-synuclein SUMOylation sites identified by mass spectrometry. Schematic depiction of a-synuclein amino acid sequence. Indicated are the amphipatic repeat region, the hydrophobic NAC region and the acidic Cterminal region of $\alpha$-synuclein. Eleven out of total fifteen lysine residues in the amino acid sequence of $\alpha$-synuclein can serve as SUMO-conjugation sites. Outlined in red are the two consensus sites K96 and K102 and in blue SUMO acceptor lysine residues, which are not part of consensus motifs.

ESI-MS/MS spectrum of the SUMO-1 conjugated a-synuclein at Lys 96, derived from a-synuclein amino acids $81-97$ is shown in Figure $3-14 a$. The measures of mass of the extrem C-terminal fragment of $\alpha$-synuclein conjugated to the SUMO at Lys 102 are shown in Figure 3-14b. The experimentally measured mass matched the calculated one (within 5 ppm) for a C-terminal peptide encompassing amino acids 98-140, SUMO conjugated at lysine 102. Unfortunately, it was not possible to obtain sufficient sequence information from this particular conjugate due to the very complex MS/MS spectra derived from the 5-times charged tryptic peptide. However, the corresponding peptide mass and the fact that $\mathrm{K} 102$ is the single lysine residue in the C-terminal fragment of a-synuclein leads to the conclusion that the second consensus lysine in a-synuclein is also SUMOylated.

In summary, eleven out of total fifteen lysine residues present in the amino acid sequence of $\alpha$-synuclein were found SUMO1 conjugated by mass spectrometric analysis. This result is in agreement with the data obtained by the mutagenesis analysis (Section 3.6.1.). 
a

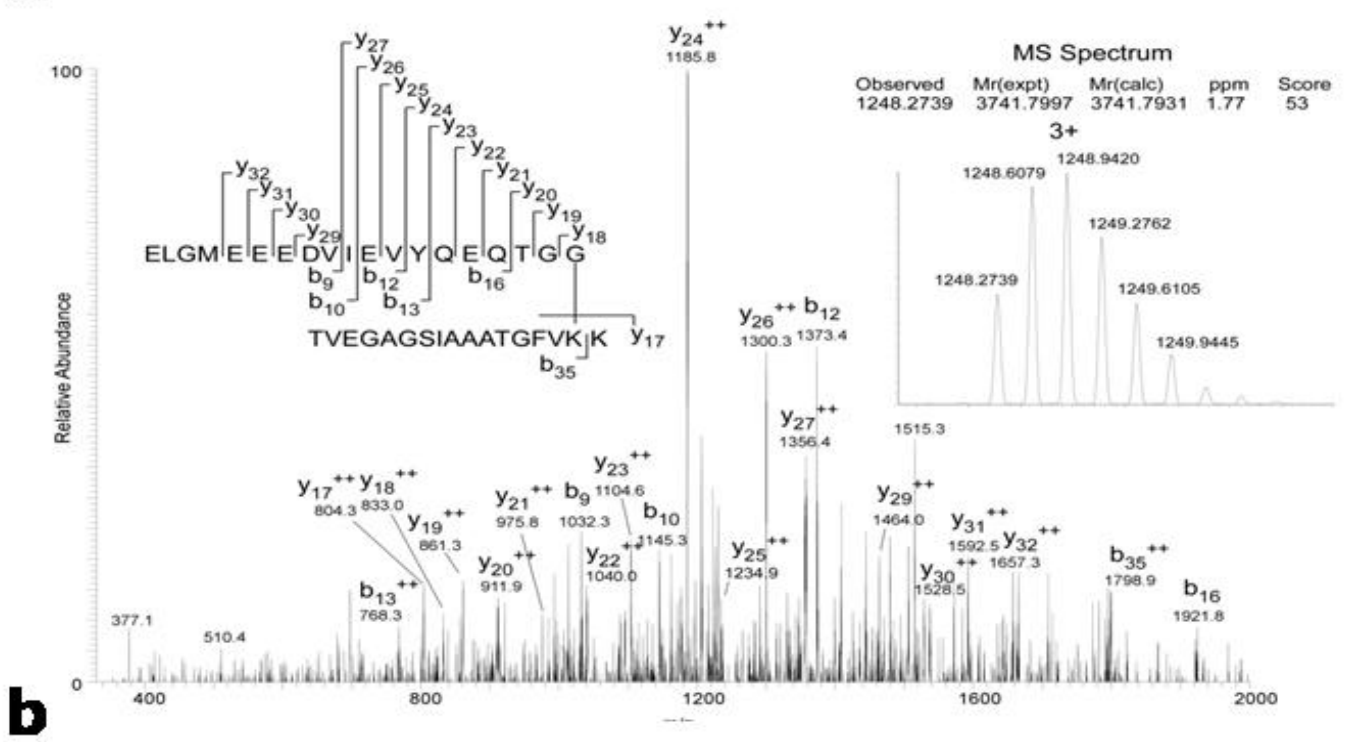

ELGMEEEDVIEVYQEQTGG

DQLGKNEEGAPQEGILEDMPVDPDNEAYEMPSEEGYQDYEPEA

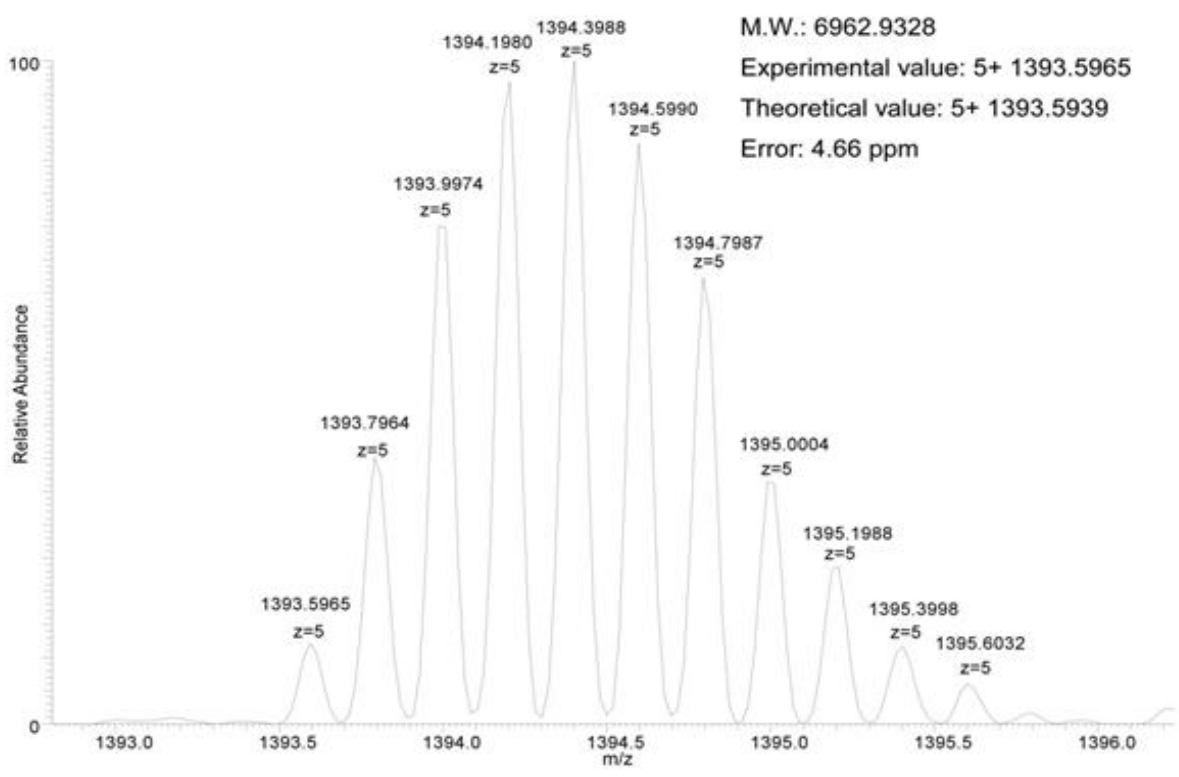

Figure 3-14. Mass spectrometry reveals SUMO conjugation at K96 and K102. (a) $\alpha$ Synuclein K96 SUMO1 conjugation mapped by mass spectrometry. ESI-MS/MS spectrum of the SUMO1 conjugated $\alpha$-synuclein at Lys $96(\mathrm{~m} / \mathrm{z}=1248.2739)$ derived from $\alpha$-synuclein encompassing positions 81-97 with fragment ions recorded in the FT-analyzer of the Orbitrap. $y$ - and b-type ions are shown in the spectrum at their respective positions in the conjugated peptide. (b) a-Synuclein-SUMO1 conjugation at K102. SI-MS spectrum of the SUMO1 conjugated $\alpha$-synuclein at Lys 102 derived from a-synuclein encompassing positions $98-140$ and recorded in the FT-analyzer of the Orbitrap. Pentuply charged isotopic signal clusters could be detected with the respective monoisotopic molecular ions occurring at $\mathrm{m} / \mathrm{z} 1393.5965$ which corresponds exactly to the theoretical value within $5 \mathrm{ppm}$. 


\subsection{Ubiquitination of $\alpha$-synuclein WT, DM and KO10 in HEK 293 cells}

Ubiquitination and SUMOylation are posttranslational modifications which use similar enzymatic pathways to conjugate substrates. In addition, lysine residues are acceptor sites for both Ubiquitin and SUMO.

a-Synuclein was found partially ubiquitinated in brains from PD patients (Hasegawa et al., 2002). It has also been shown that it can be ubiquitinated in vitro and in cell culture models. Lysine residues $21,23,32,34$ within the N-terminal repeats are liable to ubiquitination in vitro, while Lysine 6, 10, 12 are the major ubiquitination sites found in vivo (Nonaka et al., 2005). We have shown that most of those lysine residues can also serve as SUMO conjugation sites. Therefore it was essential to check the ubiquitination state of $\alpha$-synuclein-DM (K96R, K102R) and $\alpha$ synuclein-KO10 (K10R, K12R, K21R, K23R, K34R, K45R, K60R, K80R, K96R, $\mathrm{K} 102 \mathrm{R})$ compared to wild type $\alpha$-synuclein, before using those mutants for functional analysis. HEK 293 cells were co-transfected with His-tagged ubiquitin and myc-tagged $\alpha$-synuclein wild type, DM or KO10 mutant forms or empty vector. Cells were lysed under denaturing conditions and $\mathrm{His}_{6}{ }_{6}$ Ubiquitin conjugates were isolated via $\mathrm{Ni}^{2+}-\mathrm{NTA}$ chromatography. Eluates were separated by SDS-PAGE, blotted on a nitrocellulose membrane and analysed with anti-myc antibody (Figure 3-15). $\alpha$ Synuclein wild type was mono- and di-ubiquitinated in HEK 293 cells. The $\alpha-$ synuclein-DM was equally ubiquitinated as the wild type. As expected, in the case of $\alpha$-synuclein-KO10 ubiquitin conjugation was greatly reduced. This shows that K96R and K102R mutations are affecting SUMOylation but not ubiquitination. Thus the functional analysis of $\alpha$-synuclein SUMOylation is primarily based on the $\alpha$ synuclein consensus site deficient mutant (DM). 


\section{Total Protein Lysate $\quad \mathrm{Ni}^{2+}$-NTA Pulldown}

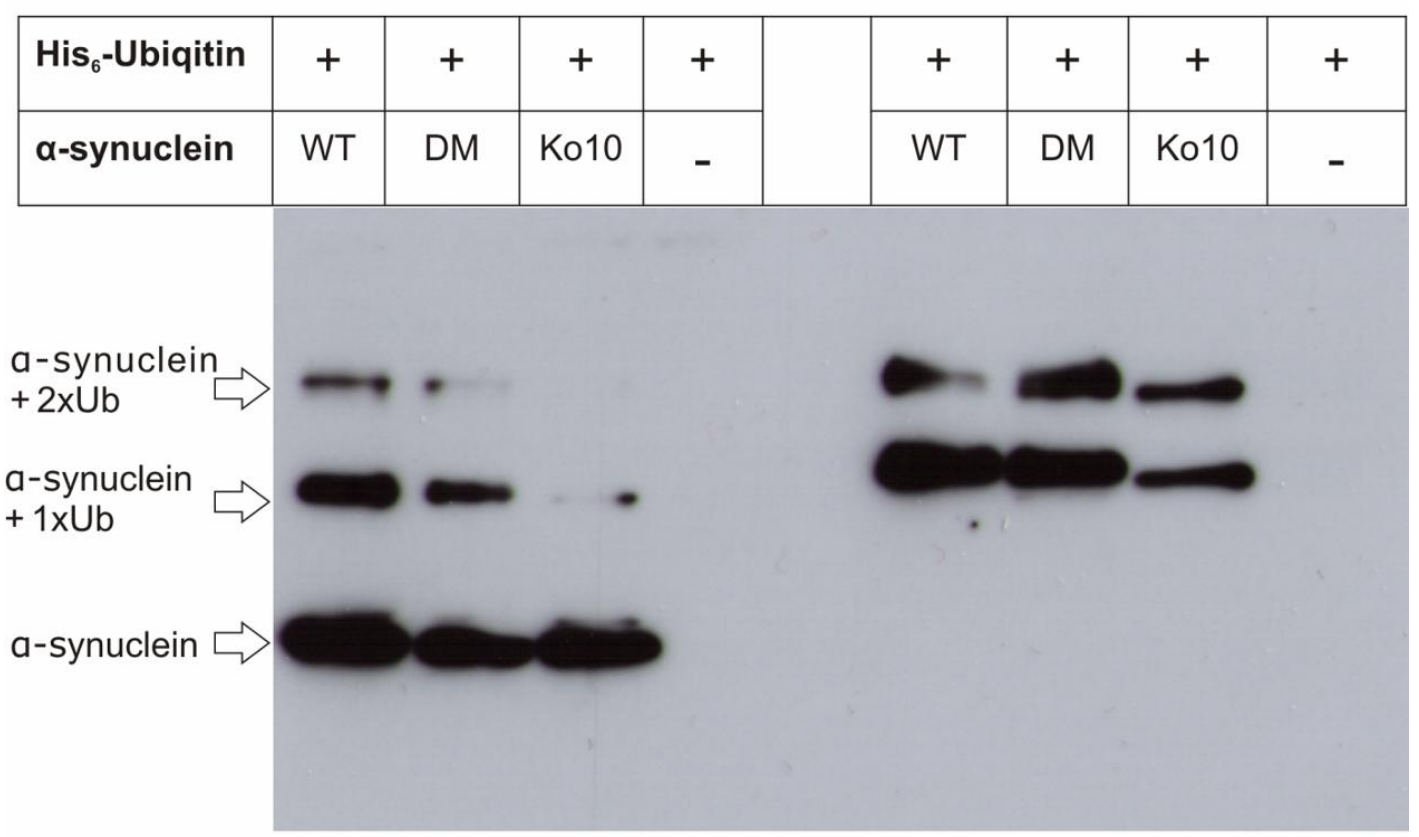

WB: anti-myc 9E10

Figure 3-15. Ubiquitination of $\alpha$-synuclein-WT, DM and KO10. HEK 293 cells were co-transfected with His-tagged Ubiquitin and myc-tagged a-synuclein wild type, mutant forms or empty vector. Cells were lysed under denaturing conditions and $\mathrm{His}_{6}$-Ubiquitin conjugates were isolated via $\mathrm{Ni}^{2+}$-NTA chromatography. Eluates were separated by SDS-PAGE, blotted on a nitrocellulose membrane and analysed with anti-myc antibody.

\subsection{In vitro fibrillation of $\alpha-s y n u c l e i n$}

One of the central disease-associated characteristics of $\alpha$-synuclein as a natively unfolded protein is its propensity to aggregate and form amyloid-fibrilcontaining inclusions in various neurodegenerative disorders. We thus aimed to test if SUMOylation has an impact on a-synuclein aggregation.

In vitro fibrillation of $\alpha$-synuclein has been well established and widely used to examine variety of factors, which can modulate $\alpha$-synuclein aggregation kinetics and eventually be tested for their therapeutic potential in different disease models. Buffer conditions, temperature, point mutations, oxidation, various posttranslational modifications, presence of other molecules (e.g. lipids) are some of the factors that can influence amyloid fibril formation in vitro. The presence of different protein tags can induce changes in the protein conformation, surface charge and solubility and interfere with the aggregation kinetics. Therefore, to study the effect of SUMOylation 
on a-synuclein fibrillation we expressed and purified non-tagged native a-synuclein in a SUMO-modified and non-modified form.

\subsubsection{Overexpression and purification of non-tagged SUMOylated $\alpha$ - synuclein}

a-Synuclein-WT and DM (K96R, K102R) were cloned into the pT7-7 vector for bacterial expression as described in section 2.2.1.11. Bacterial cells were transformed with $\alpha$-synuclein (WT or DM) plasmid and the tri-cistronic pT-E1E2S1 construct, driving the expression of E1, E2 and SUMO1 and thus reconstituting the SUMOylation machinery (without E3 ligase) in E.coli. Cells were grown under the selection of Ampicillin and Chloramphenicol. Expression was induced at $\mathrm{OD}_{600} 0,5$ 0,6 as described in the methods section 2.2.7.1. Cells were lysed in $10 \mathrm{mM}$ Tris $\mathrm{pH}$ 8.0, 1 mM EDTA pH 8.0 with an emulsion flex. SUMOylated $\alpha$-synuclein is stable and remained soluble throughout the heat denaturation $\left(95^{\circ} \mathrm{C}\right.$ for $\left.20 \mathrm{~min}\right)$ of host cell proteins and was efficiently precipitated with ammonium sulphate together with the non-modified synuclein. The SUMO-modified form has a higher negative charge (-13.1 at $\mathrm{pH} 7.0)$ compared to free synuclein (-8.8 at $\mathrm{pH} 7.0)$ and binds anionexchange matrix stronger. The majority of non-modified synuclein was eluted in fractions 16-20 (Figure 3-16, A) and SUMOylated $\alpha$-synuclein was eluted from the column at higher salt concentrations (fractions 24-27, Figure 3-16, A).

This allowed us to use a similar purification procedure for SUMO conjugated $\alpha$ synuclein, as for the non-modified one (described in section 2.2.7.1.) with some alterations. Although SUMO-conjugated $\alpha$-synuclein has a higher binding affinity to Mono $Q$ resin than 'free' $\alpha$-synuclein, some non-modified $\alpha$-synuclein was co-eluted with the SUMO-synuclein. To enrich purity and yield of the SUMOylated form, all fractions containing SUMO-modified a-synuclein were pooled, concentrated, desalted and subjected to a second MonoQ chromatography run (Figure 3-16, B). 
A

Anion exchange chromatography: Run1

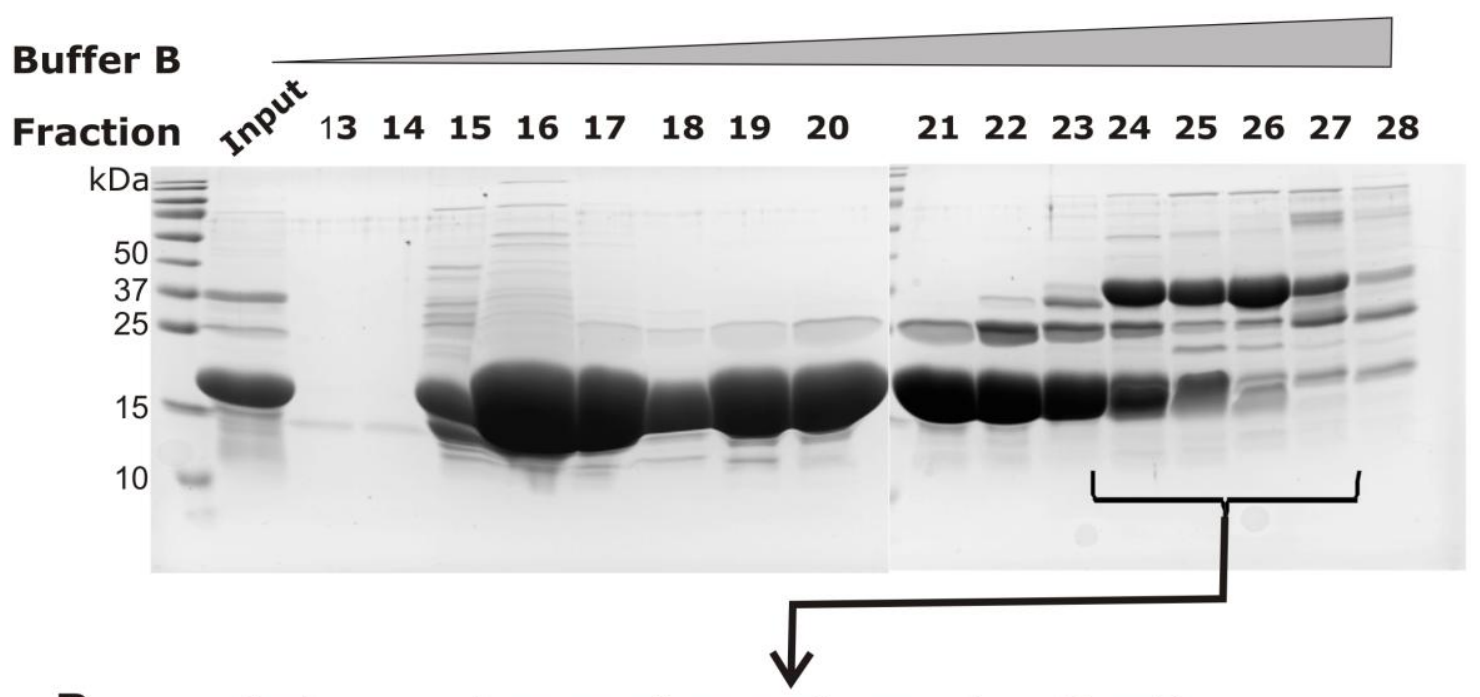

B Anion exchange chromatography: Run2

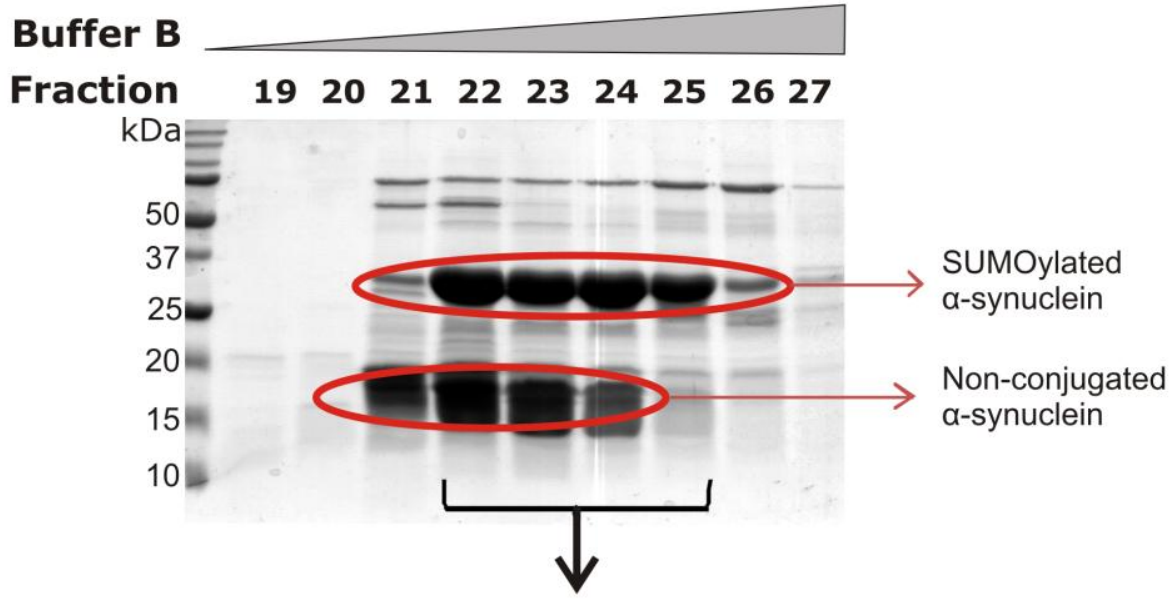

C

\section{Gel Filtration (Superdex 75)}

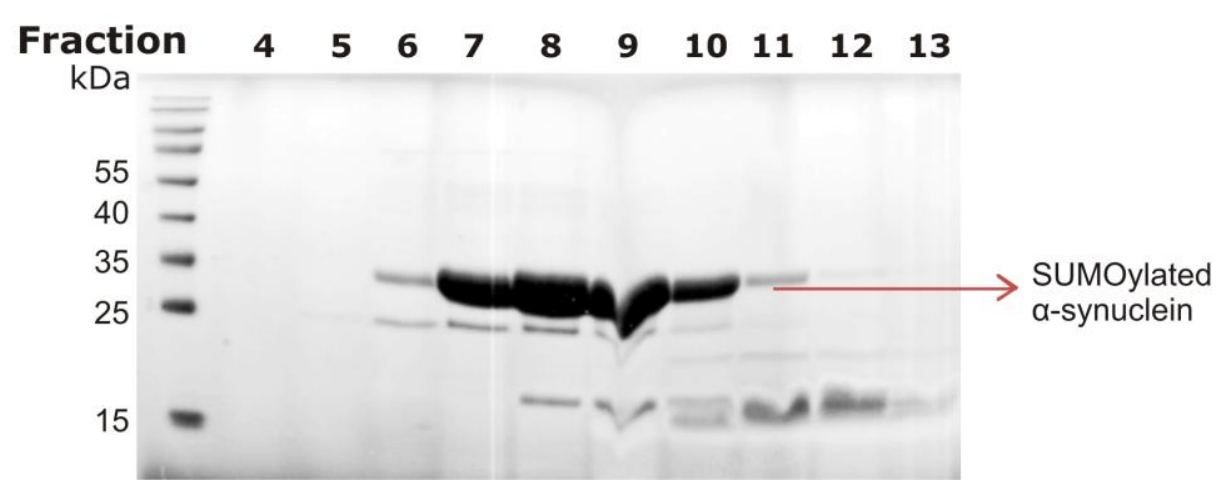

Figure 3-16. Purification of non-tagged SUMOylated a-synuclein. Crude lysate after heat denaturation (input) was loaded on anion-exchange column (Mono Q). Bound proteins were eluted with increasing concentrations of Buffer $B(1 \mathrm{M} \mathrm{NaCl}, 25 \mathrm{mM}$ Tris $\mathrm{pH}$ 7.7) and fractions containing non-modified $\alpha$-synuclein and the SUMOylated form were collected (A). Fractions 24-27 were pooled and SUMOylated $\alpha$-synuclein was enriched via a second anion-exchange chromatography run (B). Fractions 2225 of the second Mono $Q$ run were pooled and further purified using gel filtration (C). 
The SUMOylated a-synuclein was further purified using gel filtration chromatography (Figure 3-16, C) for elimination of residual 'free' synuclein. Elution fractions 7-9 contained 95\% pure SUMOylated a-synuclein and were used in aggregation assays. This purification procedure was applied for overexpression and production of SUMOylated $\alpha$-synuclein wild type and DM (K96R, K102R).

\subsubsection{SUMOylation inhibits $\alpha$-synuclein fibrillation in vitro}

SUMO is an extremely soluble protein and it has been proven to increase the solubility of recombinantly expressed proteins once fused to them. However, there are very few studies investigating the effect of conjugated SUMO on target solubility using mutants deficient in the SUMO conjugation site. We decided to investigate the effect of conjugated SUMO on a-synuclein fibrillization using an in vitro aggregation assay with purified proteins (Figure 3-17).

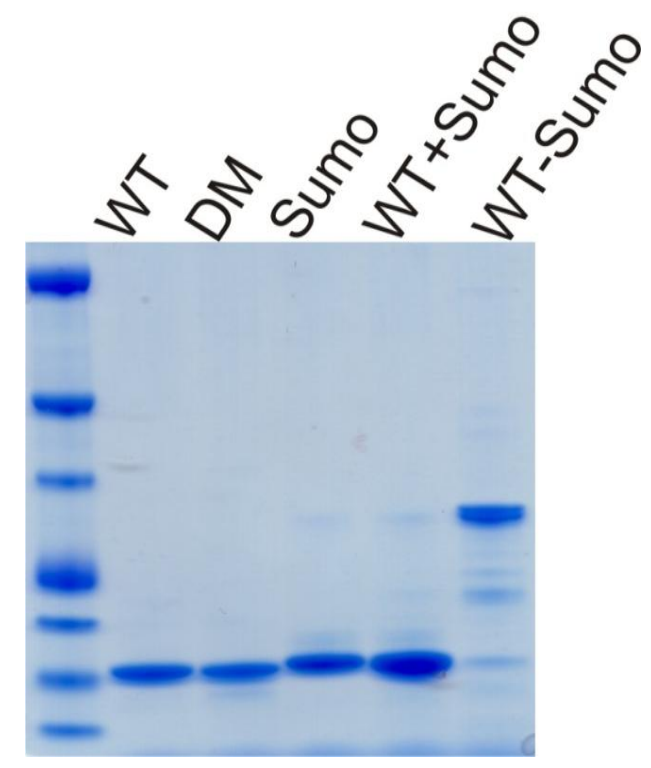

Figure 3-17. Coomassie staining of recombinantly purified proteins used in the in vitro fibrillization assay. a-Synuclein wild type (WT); a-synuclein-DM (DM); equimolar mixture of $\alpha$-synuclein and SUMO1 (WT+SUMO); SUMOylated $\alpha-$ synuclein (WT-SUMO).

When monomeric a-synuclein is incubated at $37^{\circ} \mathrm{C}$ and $\mathrm{pH} 7.4$ it forms amyloid fibrils. These are very similar to $\alpha$-synuclein filaments isolated from patients with synucleinopathies (Crowther et al., 2000). Recombinantly expressed and purified wild type a-synuclein (Syn-WT), a-synuclein-DM (Syn-DM), SUMOylated wild type a-synuclein (SUMOylated Syn-WT), SUMOylated a-synuclein-DM 
(SUMOylated Syn-DM), an equimolar mixture of a-synuclein-WT and SUMO (SynWT+SUMO) and SUMO were incubated at concentrations of $70 \mu \mathrm{M}$ in $50 \mathrm{mM}$ Hepes, $100 \mathrm{mM} \mathrm{NaCl}, \mathrm{pH} 7.4$ at $37^{\circ} \mathrm{C}$ with constant agitation. Fibrillization was monitored by enhancement of ThioflavinT fluorescence upon its binding to the cross- $B$ structures of the amyloid fibrils (Figure 3-18).

As expected, fibrillization of wild type $\alpha$-synuclein increased with time reaching a saturation level after approximately $72 \mathrm{~h}$ of incubation. Mutations of the two SUMO consensus lysines in $\alpha$-synuclein did not interfere with fibril formation and similar aggregation kinetics were observed for the $\alpha$-synuclein-DM. The presence of SUMO as an equimolar component of synuclein-SUMO mixture did not prevent fibril formation. In striking contrast, purified SUMOylated a-synuclein wild type and DM exhibited no detectable fibril formation after $92 \mathrm{~h}$ of incubation. No fibril formation was observed in the sample containing purified SUMO only. This result shows that covalent conjugation of single molecule SUMO to a-synuclein prevents it from fibril formation in vitro. SUMOylation inhibits $\alpha$-synuclein amyloid formation even when SUMO is not conjugated to one of the two consensus lysine residues but to one of the lysines that do not fall into a consensus motif.

Transmission electron microscopy (TEM) micrographs confirm amyloid fibril formation for a-synuclein wild type and DM as well as the mixture of both SUMO and a-synuclein (Figure 3-19). 


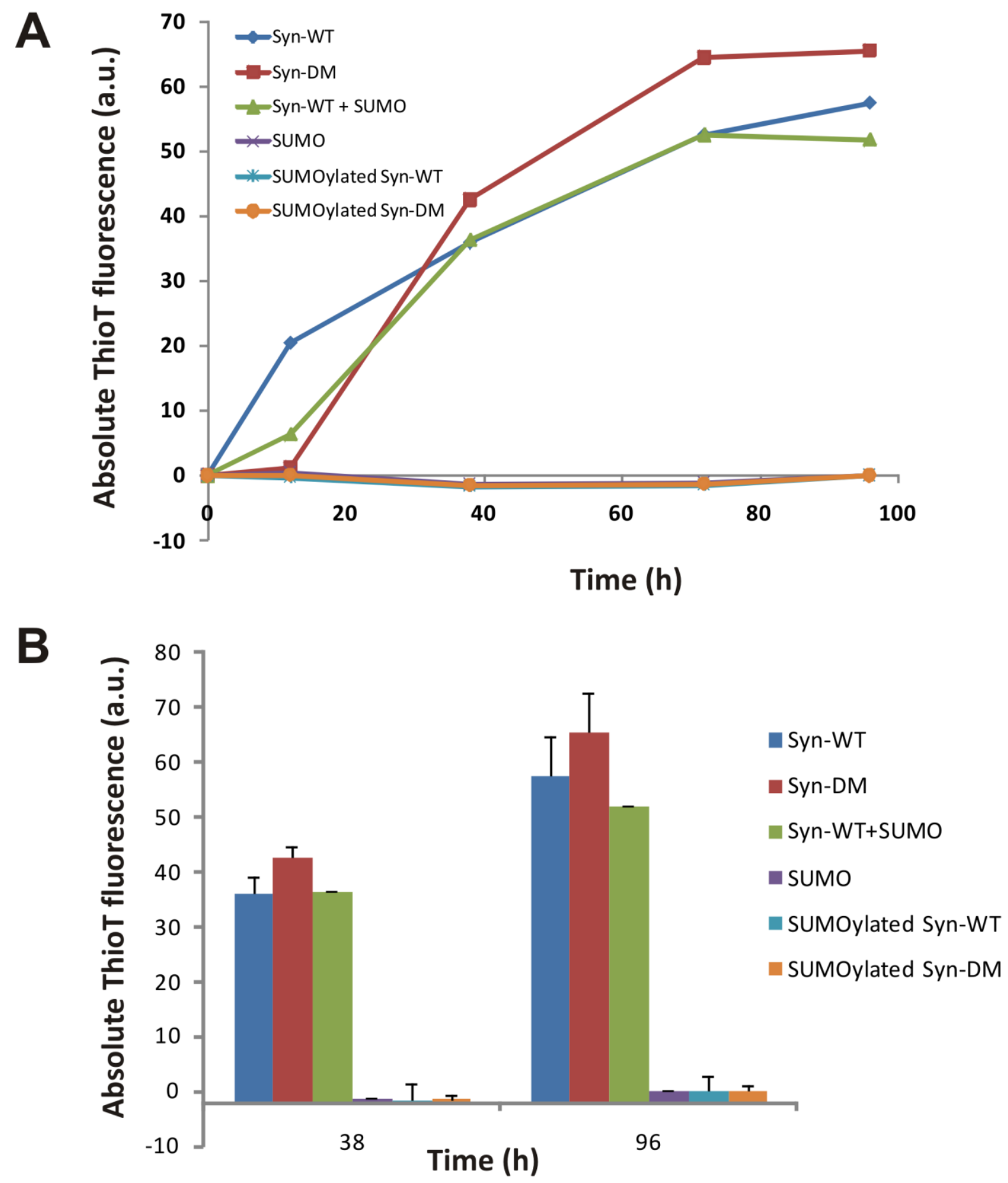

Figure 3-18. In vitro fibrillization of $\alpha$-synuclein. (A) Kinetis of fibrillization of wild type a-synuclein (Syn-WT), a-synuclein-DM (Syn-DM), SUMOylated wild type $\alpha$ synuclein (SUMOylated Syn-WT), SUMOylated a-synuclein-DM (SUMOylated SynDM), equimolar mixture of wild type a-synuclein and SUMO (Syn-WT+SUMO) and SUMO alone as monitored by Thioflavin-T (ThioT) fluorescence over time. (B) Bar graph representation of the fibril formation in all tested conditions for two different time points (after $38 \mathrm{~h}$ and $96 \mathrm{~h}$ incubation). Data are represented as mean $\pm \mathrm{SD}$. 


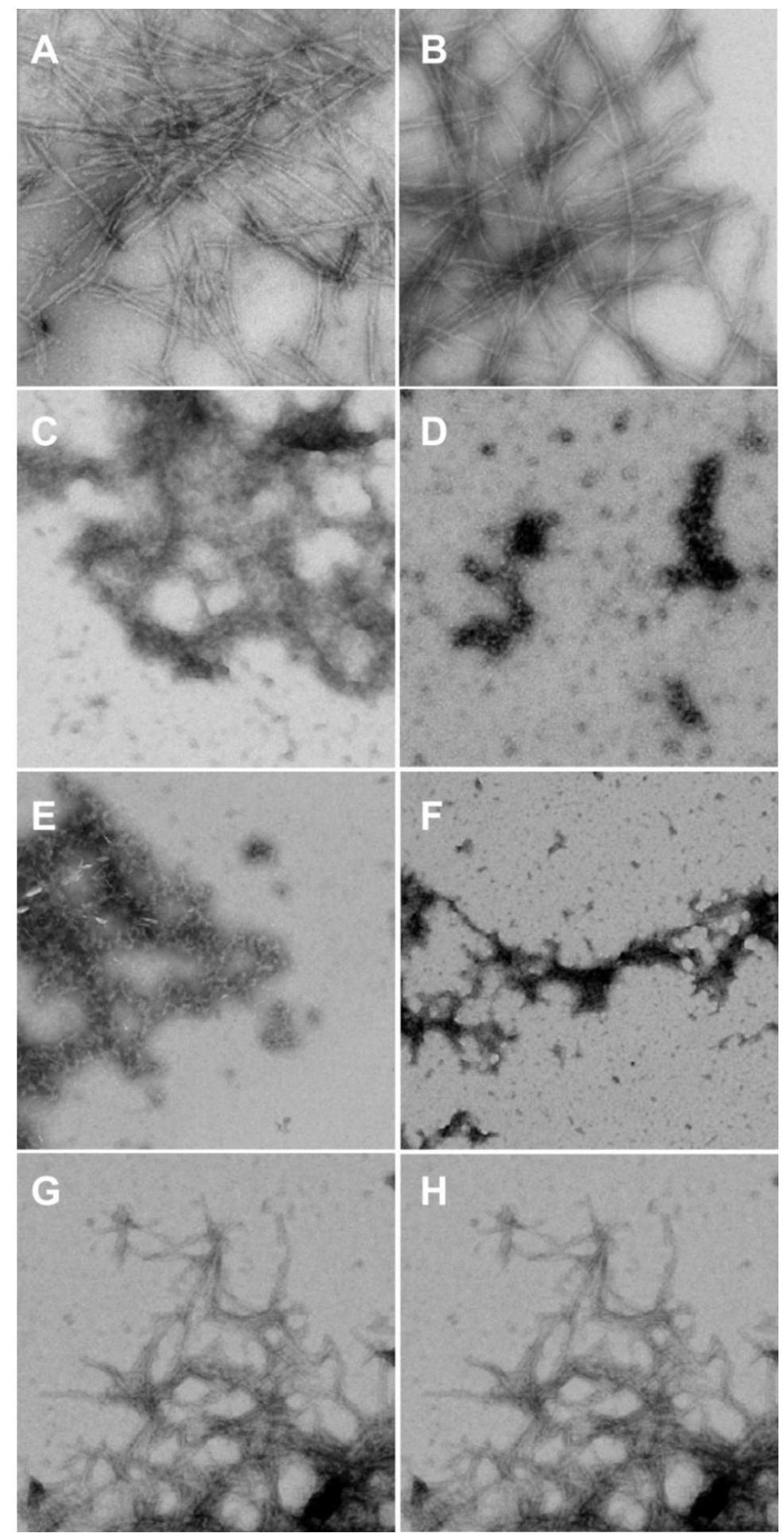

Figure 3-19. Transmission electron microscopy (TEM) of in vitro aggregation samples after $96 \mathrm{~h}$ incubation in $50 \mathrm{mM}$ Hepes, $100 \mathrm{mM} \mathrm{NaCl}$, and $\mathrm{pH} 7.4$ at $37^{\circ} \mathrm{C}$ with stirring. Mature fibrils formed by $\alpha$-synuclein-WT (A) and $\alpha$-synuclein-DM (B). Nonamyloidogenic amorphous aggregates are formed by SUMOylated $\alpha$-synuclein-WT $(C, D)$ and SUMOylated $\alpha$-synuclein-DM (E, F). Mixture of SUMO and wild type $\alpha$ synuclein resulted in the formation of smaller in size but mature fibrils $(G, H)$.

a-Synuclein-WT and DM formed morphologically identical mature amyloid fibrils suggesting that the K96R and K102R mutations have no obvious effect on $\alpha$ - 
synuclein fibrillation in vitro. TEM revealed non-amyloidogenic amorphous aggregates in the SUMOylated WT and DM samples. Those inclusions are devoid of B-sheet rich structures and therefore give no Thioflavin-T fluorescence signal. The presence of non-conjugated SUMO in the a-synuclein-SUMO mixture led to formation of mature but smaller-sized fibrils.

The aggregation assays were performed by Pinar Karpinar and Dr. Markus Zweckstetter (NMR-based Structural Biology, Max-Planck Institute for Biophysical Chemistry).

\subsection{The role of $\alpha$-synuclein SUMOylation in dopaminergic cell survival}

Together with aggregate formation, neurotoxicity is the second disease related hallmark of $\alpha$-synuclein. Overexpression of wild type $\alpha$-synuclein resulting from gene duplication is sufficient to cause Parkinson's disease. Expression of $\alpha$ synuclein has been shown to cause dopaminergic cell death in various disease models.

To investigate the impact of a-synuclein SUMOylation on the survival of dopaminergic neurons in vivo we tested two SUMOylation related mutants of $\alpha$ synuclein - DM and KO10 in the rat model of Parkinson's disease. $\alpha$-Synuclein-DM has both consensus SUMOylation sites, Lys96 and Lys102, mutated to arginine which in cell culture pulldown experiments led to about $50 \%$ reduction in SUMO conjugation. The second mutant $\alpha$-synuclein-KO10 has 10 lysine residues, which were confirmed as SUMO conjugation sites in mass spectrometry experiments, mutated to arginine and leads to much stronger reduction in SUMOylation (shown in Results section 3.5). Recombinant adeno-associated viral vectors (rAAV serotype 2) were used as gene delivery vehicle of wild type and mutant forms of a-synuclein. rAAVs are not associated with any known human disease and have neither shown inherent vector toxicity nor immunogenicity. The AAV genome is devoid of all viral genes with the exception of the short inverted terminal repeats for viral genome packaging (Peel and Klein, 2000). AAV serotype 2 has been shown to have neuron restricted tropism in the adult rat brain (Bartlett et al., 1998).

The genome structure of the constructed viral vectors is shown in Figure 320. All transgenes were expressed under the human synapsin 1 (hSyn1) promoter, which has been shown to restrict the transgene expression exclusively to neurons in 
vitro and in vivo (Kugler et al., 2001). Another benefit from using rAAVs is the longlasting transgene expression.

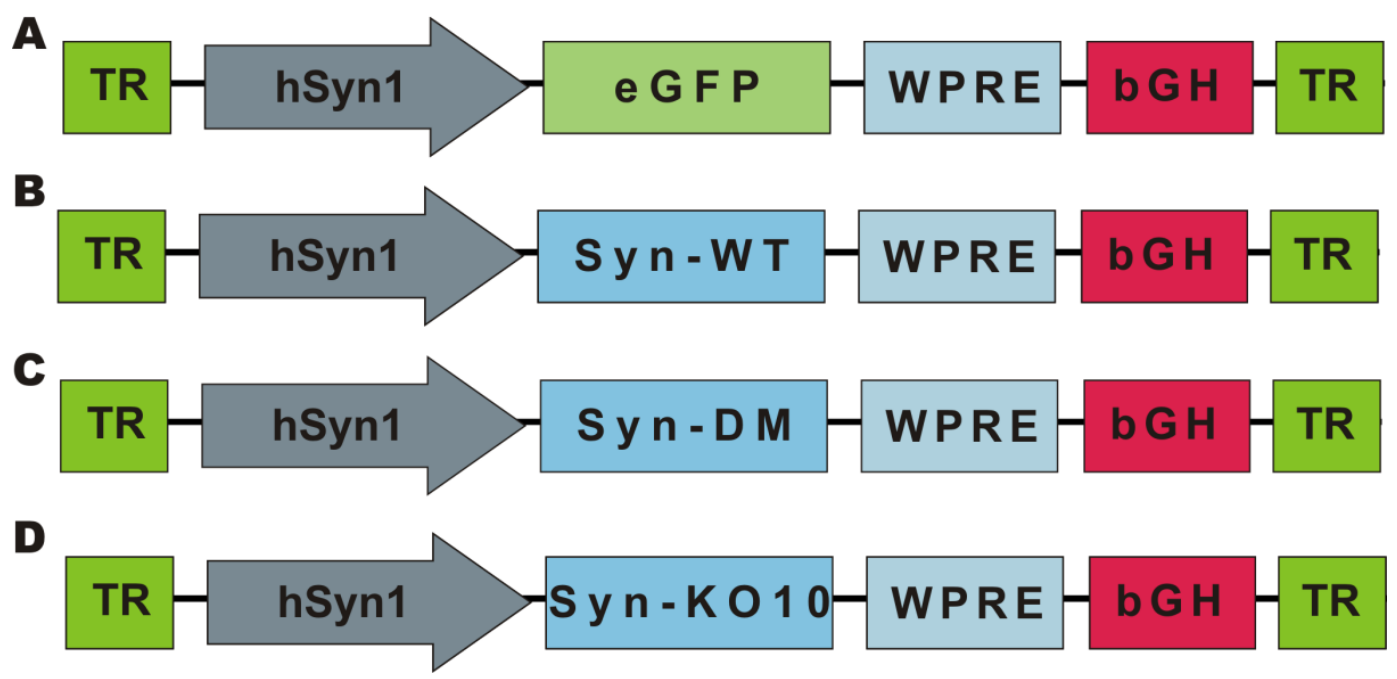

Figure 3-20. Schematic representation of vector genomes used in the in vivo rat model of PD. AAV2 vectors expressing eGFP (A), a-synuclein wild type (B), $\alpha-$ synuclein-DM (C), $\alpha$-synuclein-KO10 (D) under the neuron specific human synapsin 1 gene promoter (hSyn1) encode also inverted terminal repeats (TR) and small control elements - WPRE, woodchuck hepatitis virus posttranscriptional control element; bGH, bovine growth hormone derived polyadenylation site.

Viral titers were confirmed in vitro by transduction of primary rat cortical neurons which were subjected to Western blot analysis of transgene expression after lysis. The rAAVs led to expression of comparable amounts of human $\alpha$ synuclein wild type and mutant forms (Figure 3-21).

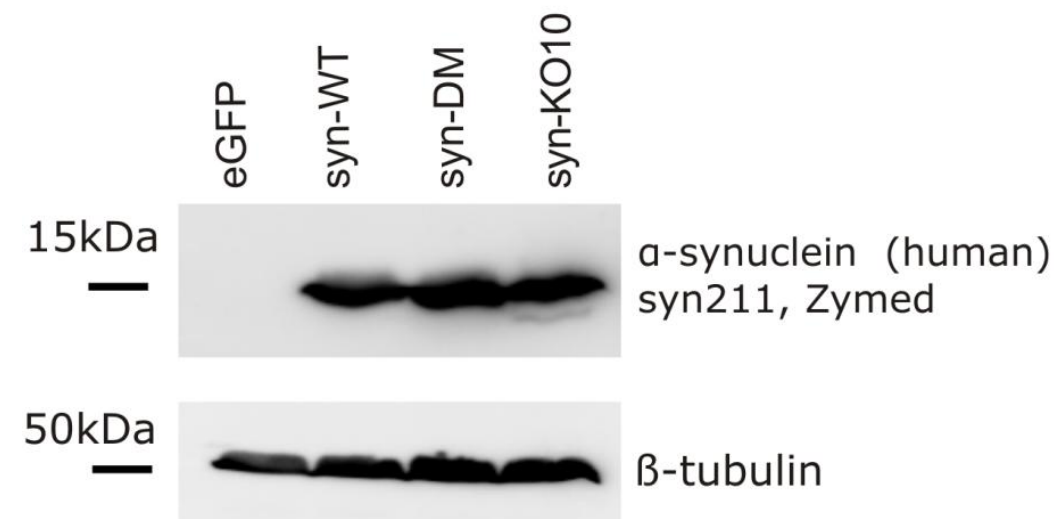

Figure 3-21. Cultured primary rat cortical neurons transduced with AAV2 vectors encoding a-synuclein WT, DM and KO10. Cortical cultures were lysed four days after transduction and $\alpha$-synuclein expression levels were analysed by Western blot with human specific antibody. 
Female Wistar rats were injected with equal amounts of rAAV vectors that expressed eGFP as a control, the human wild-type a-synuclein protein, DM or KO10 SUMOylation deficient mutants. Stereotactical injections were done unilaterally in the substantia nigra pars compacta (SNpc) to determine the effect of transgene expression on degeneration of dopaminergic neurons; the other brain hemisphere was kept as a nontransduced internal control. Animals were sacrificed and perfused 12 weeks after injections. This time point was selected based on previous results, which demonstrated that expression of a-synuclein reached a maximum at 3-4 weeks after nigral injection and neural degeneration became significant at 8-10 weeks (Kirik et al., 2002). 

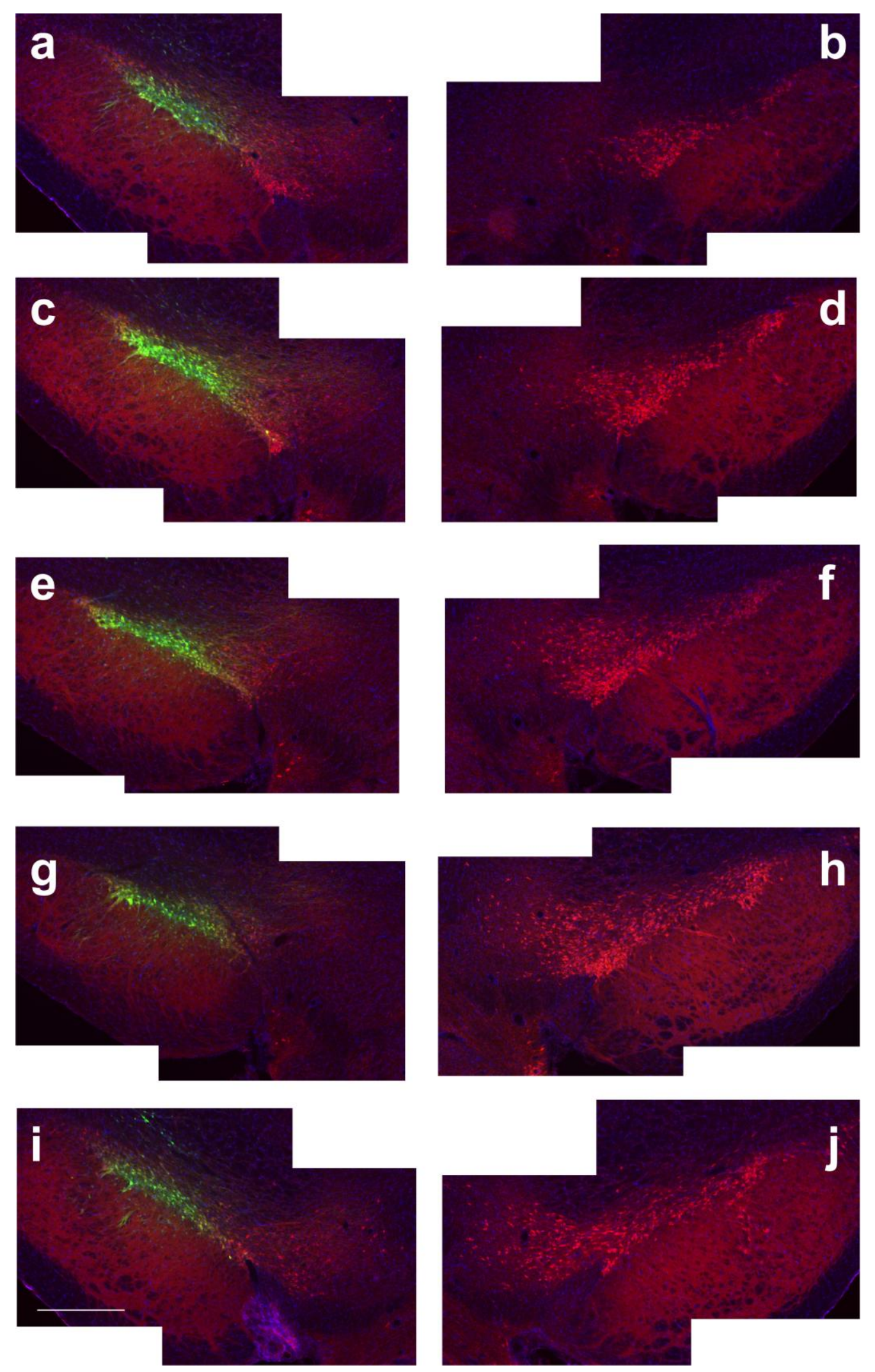

Figure 3-22. Unilateral rAAV-mediated transduction of SNpc. Fluorescence microscopy of VMAT2 positive neurons. Photomicrographs showing VMAT2 positive neurons (red) in the left $(a, c, e, g, i)$ and right side $(b, d, f, h, j)$ of the midbrain. Expression of the GFP transgene (green) was detected only in the injected left side of the brain. The majority of the VMAT2 positive cells in SNpc are also GFP positive. (Scale bar: $0,5 \mathrm{~mm}$ ) 
Control injections with AAV2 expressing eGFP showed GFP-positive (GFP $\left.{ }^{+}\right)$ cell bodies in the majority of VMAT2 (Vesicular Monoamine Transporter 2) positive neurons of the SNpc (Figure 3-22). However, the transduction area of $\mathrm{GFP}^{+}$ neurons was not limited to the SNpc, because there were $\mathrm{GFP}^{+}$neurons in substantia nigra pars reticulata and in a variable number of cells in the ventral tegmental area (VTA).

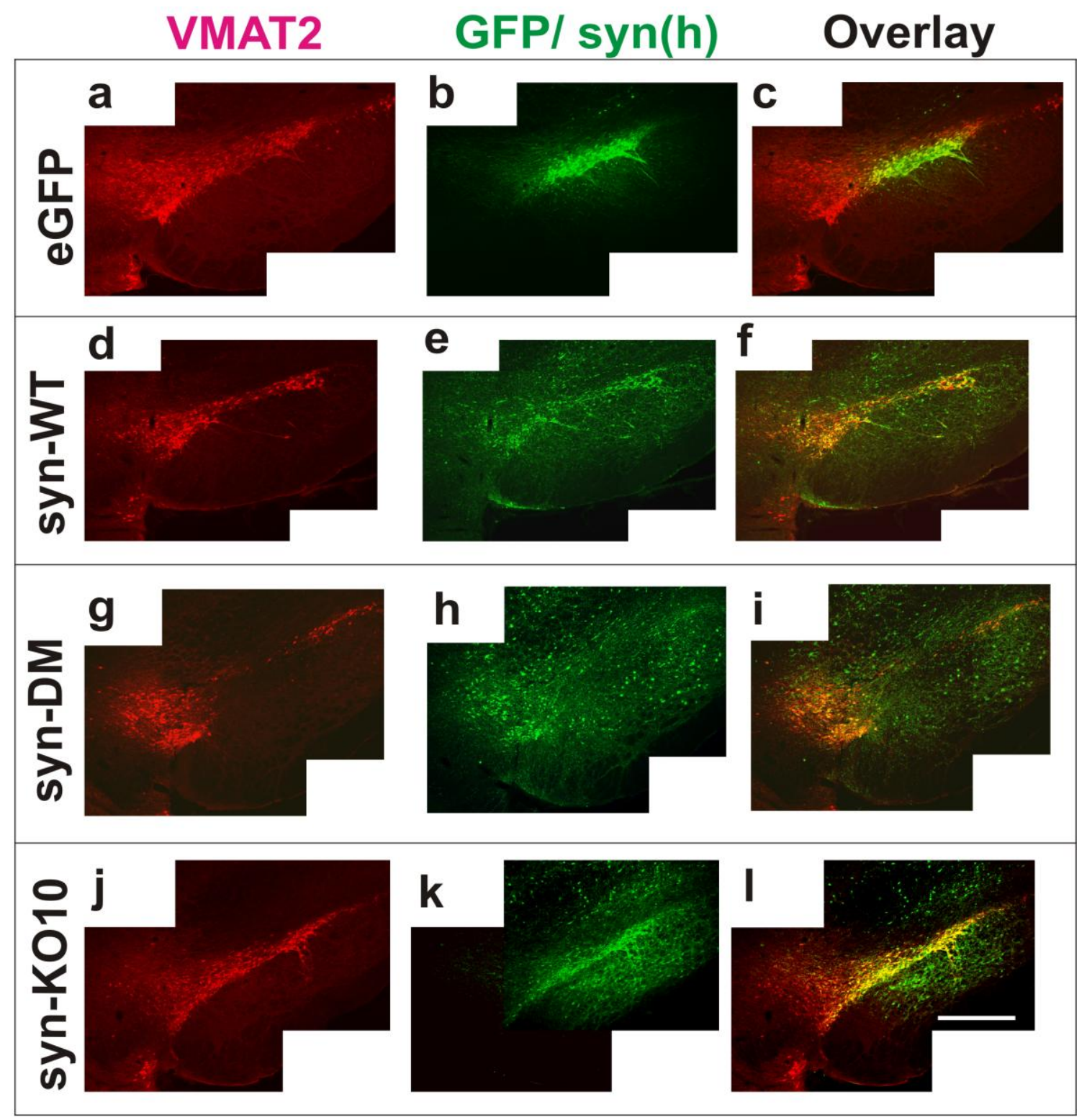

Figure 3-23. Photomicrographs showing nigral degeneration in rAAV- $\alpha$-synucleininjected animals. Each row contains a representative $S N$ section form each animal group - rAAV-eGFP (a,b,c); rAVV-synuclein-WT (d,e,f); rAAV-synuclein-DM (g,h,i) and rAAV-synuclein-KO10 (j,k,l). Sections were stained for VMAT2 (a,d,g,j) and GFP(b) or human $\alpha$-synuclein $(e, h, k)$. The overlays show co-expression of VMAT2 and GFP/a-synuclein in SN 12 weeks post-injection (c,f,i,l) and loss of nigral cells in the rAAV-synuclein WT/DM injected.animals $(f, i)$. (Scale bar: 0,5 mm) 
A similar pattern of expression was seen in the rAAV- $\alpha$-synuclein injected animals, revealed by immunostaining with an antibody recognizing wt and mutant human, but not rodent $\alpha$-synuclein (Figure 3-23).

To evaluate the toxicity of $\alpha$-synuclein wild type and mutant forms, 6-8 animals were injected per group and sacrificed twelve weeks post-injection. The brain region containing SNpc was cut in $30 \mu \mathrm{m}$ thick cryosections. Every third section was immunostained for the dopaminergic cell marker VMAT2 and unbiased blinded cell counts were obtained using Stereo Investigator 6.0 software. VMAT-positive cells restricted to the SNpc regions, but not SNpr and VTA, were taken into account. Comparison of the number of $\mathrm{VMAT2}^{+}$neurons in the rAAVeGFP injected SNpc and the non-injected (NI) contralateral SNpc showed no significant cell loss in the eGFP injected control group (14037.3 $\pm 319 \mathrm{VMAT2}^{+}$cells in $\mathrm{NI}$ compared to $13505 \pm 201$ surviving $\mathrm{VMAT2}^{+}$cells in the rAAV-eGFP control; n.s.) (Figure 3-23, 3-24). In contrast to rAAV-eGFP vehicle control, the number of $\mathrm{VMAT}^{+}$cells of rAAV-synuclein-WT and the two mutants were significantly reduced $\left(p<0.01^{* \star}\right)$. Viral vector expression of wild type human $\alpha$-synuclein resulted in survival of $\approx 58 \%$ of the $\mathrm{VMAT}^{+}$cells compared to the eGFP control group (7896.7 \pm 1007 surviving VMAT2 $^{+}$cells in rAAV-synuclein-WT compared to rAAVeGFP control; $\left.p<0.01^{* *}\right)$. The two lysine substitutions K96R and K102R in rAAVsynuclein-DM exacerbated its neurodegenerative effect and led to loss of $\approx 66 \%$ of the $\mathrm{VMAT}^{+}$cells in the transduced nigral regions (4576.5 \pm 704 surviving dopaminergic SNpc neurons; $\left.p<0.01^{* \star}\right)$. Surprisingly, the animals injected with rAAV-synuclein-KO10 which has ten lysine to arginine mutations, including Lys96 and Lys102, showed much less pronounced loss of $\mathrm{VMAT}^{+}$neurons $(\approx 21 \%$; $10731 \pm 1551$ surviving dopaminergic SNpc neurons; $\left.p<0.01^{\star \star}\right)$, compared to the $\alpha$ synuclein-DM and even $\alpha$-synuclein wild type ones. 


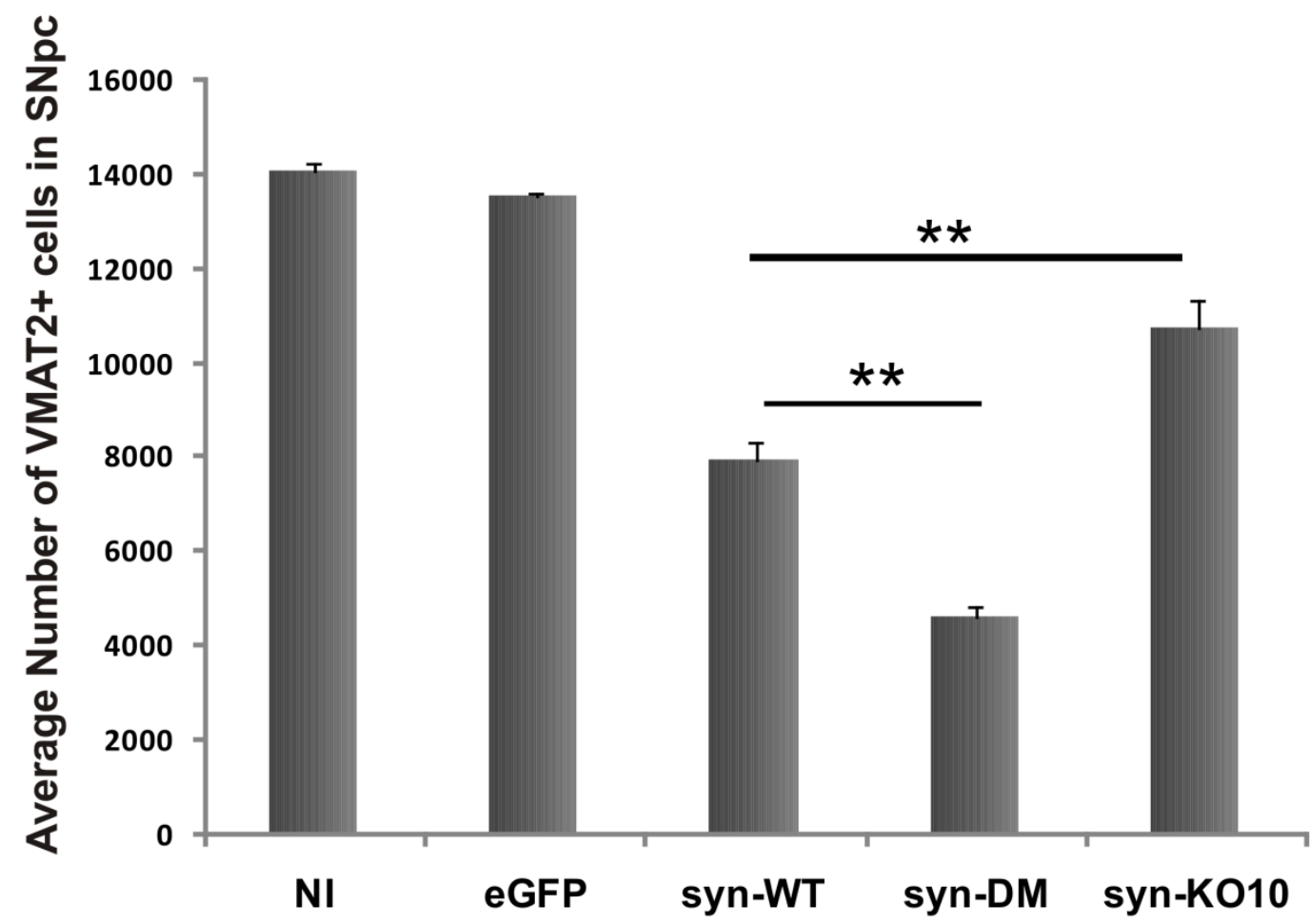

Figure 3-24. Unbiased estimation of VMAT2+ cells in the SNpc of animals injected with wild type or mutant human a-synuclein. Brain tissue sections of $30 \mu \mathrm{m}$ thickness were labelled with VMAT2 and $\alpha$-synuclein and $\mathrm{VMAT2}^{+}$cells were stereologically counted (described in 2.2.11 and 2.2.12). The graph displays the comparison of dopaminergic cell survival in eGFP, a-synuclein-WT, DM and KO10 injected animals. (NI: contralateral non-injected side). Results are average \pm S.E.M: NI - 185; eGFP - 90; syn-WT -411; syn-DM - 287; syn- KO10 - 633; $\mathrm{p}<0.01^{* \star}$ Student $\mathrm{T}$ test.

It has to be taken into account that $\alpha$-synuclein-KO10 has 10 amino acid substitutions, which could modify essential a-synuclein characteristics and potentially lead to loss of a-synuclein functions necessary for its dominant toxic effect. Moreover, we have shown that ubiquitination is strongly reduced in the case of $\alpha$-synuclein-KO10, but not for $\alpha$-synuclein-DM. Therefore for studying the specific role of SUMOylation in a-synuclein toxicity and pathology in vivo, the more SUMOconjugation specific mutant that needs to be considered is the a-synuclein-DM.

\subsection{0. $\alpha-S y n u c l e i n-D M$ has a higher aggregation rate and is more toxic in HEK}

\section{3 cells.}

We then used an in vitro model to directly correlate a-synuclein induced toxicity and aggregation in the context of SUMOylation. 
Visualization of $\alpha$-synuclein aggregate formation using directly fused fluorescent tags has proven to be a challenge in various cell line models (McLean et al., 2001). Moreover, large fluorescent proteins like GFP can sterically hinder $\alpha$ synuclein aggregation and substantially increase its solubility. To characterize and compare $\alpha$-synuclein wild type and DM aggregate formation and toxicity, we employed a newly developed $\alpha$-synuclein PDZ-domain-based fluorescence assay (Opazo et al., 2008).

Here, $\alpha$-Synuclein WT or DM was tagged with a 6 amino acid PDZ binding motif (HSTTRV from neuroligin 1) and coexpressed with the corresponding PDZ domain (PDZ1 domain of S-SCAM) fused to GFP. To ensure equal expression levels and eliminate differences in transfection efficiencies a-synuclein WT or mutant tagged with the PDZ binding motif were subcloned in a bicistronic plasmid together with PDZ-GFP fusion under the same promoter (CMV). The advantage of this approach is that a fluorescent protein is not directly fused to $\alpha$-synuclein and at the same time inclusions formed by a-synuclein can be GFP-labelled via the PDZ binding motif (Opazo et al., 2008).

HEK 293 cells were transiently transfected with $\alpha$-synuclein and PDZ-GFP. Four patterns of GFP distribution were observed - homogenous appearance (Figure 3-25,a), cells with one, usually perinuclear aggregosome (Figure 3-25,b), cells having more than one, usually small aggregates (Figure 3-25,c) and preapoptotic cells (Figure 3-25,d). Cells having more than one aggregate contained in most cases an aggregosome as well; therefore they were characterized as cells with 'many aggregates'. We used the PDZ-based assay to evaluate and compare the aggregation formation and toxicity of wild type and consensus SUMO-sitedeficient $\alpha$-synuclein. 

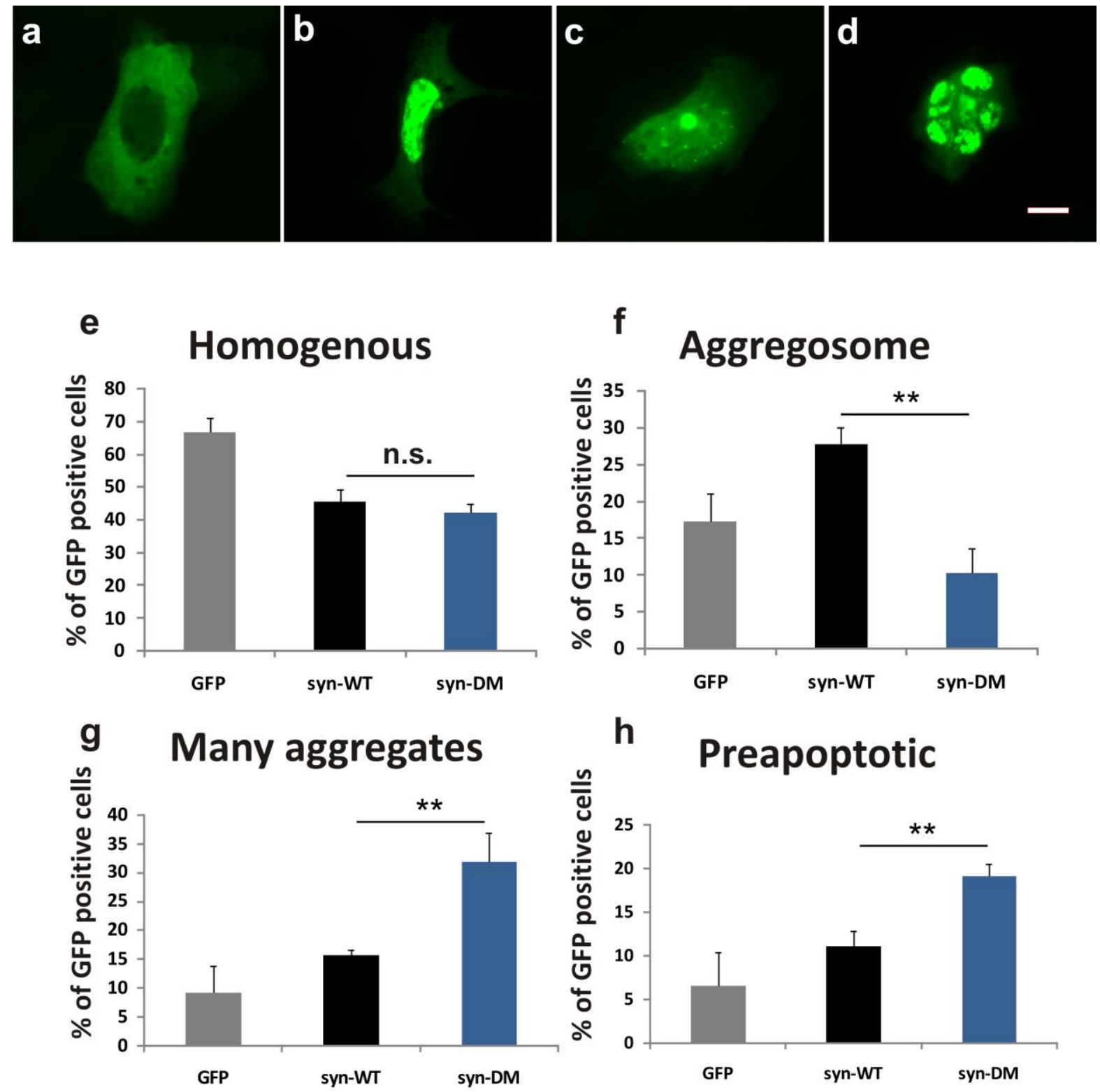

Figure 3-25. Comparison of GFP distribution patterns between $\alpha$-synuclein wild type or DM using the PDZ assay. The PDZ binding motif HSTTRV was used as a tag fused to $\alpha$-synuclein WT or DM together with GFP fused to the PDZ domain in a bicistronic vector. Cells were transfected with: PDZ-EGFP alone (Ctrl); $\alpha$-synuclein wild type tagged with the PDZ binding motif and PDZ-EGFP (syn-WT); $\alpha$-synucleinDM (K96R, K102R) PDZ tagged and PDZ-EGFP (syn-DM). Cells were classified in four groups: (a) 'homogenous', (b) 'aggregosome', (c) 'many aggregates' or (d) 'preapoptotic'. Bar graphs represent percentages of all GFP-positive cells in the four categories $(e, f, g, h)$, summarized as mean \pm SEM. Comparisons were made with one-way Student's T-test $\left(p<0.01^{* *}\right)$. Scale bar: $20 \mu \mathrm{m}$.

Cells transfected with PDZ motif and PDZ-GFP were used as a control condition. There was no significant difference in the percentage of homogenous cells transfected with wild type or DM a-synuclein. The number of cells, forming a single perinuclear aggregosome, was two times more in the samples transfected with wild 
type a-synuclein compared to DM. The reverse situation was observed in the cell population containing many dispersed aggregates. Wild type a-synuclein led to formation of multiple aggregate in $\approx 15 \%$ of the GFP positive cells, while the $\alpha$ synuclein-DM transfected samples had $\approx 35 \%$ GFP positive cells with more than one aggregate.

In summary, mutations in the two SUMO consensus sites of a-synuclein induced the redistribution of aggregates from single prominent aggregosome to disperse multiple cytosolic inclusions which correlated with increased cytotoxicity of a-synuclein-DM in vitro. 


\section{Discussion}

a-Synuclein is a small presynaptic protein associated with several neurodegenerative disorders, the most common of which is Parkinson's disease. Its physiological function is poorly understood. However being the most abundant constituent of Lewy body inclusions, a pathological role has been suggested for $\alpha$ synuclein. Indeed, point mutations in a-synuclein or mere overexpression of the wild type species can cause PD. Even though the exact molecular mechanisms leading to $\alpha$-synuclein toxicity are still unknown, abnormal folding and processing of $\alpha$ synuclein are considered as initial events for aggregation and fibril formation.

Eukaryotic cells have developed efficient ways to regulate protein function and fate via posttranslational attachment of small chemical groups like acetyl, methyl and phosphate moieties or larger molecules like Ubiquitin and SUMO. a-Synuclein is subject to various posttranslational modifications including phosphorylation, ubiquitination, oxidation and nitrosylation. In the present study we demonstrate that a-synuclein can be posttranslationally modified by SUMO1 and SUMO2. Additionally, we show a novel function of SUMO as modifier of a-synuclein fibril formation and regulator of $\alpha$-synuclein toxicity in vivo.

\section{1. $\alpha$-Synuclein is modified by SUMO}

Employing various techniques including in vitro enzymatic assays, affinity purification of covalently modified substrates from cell culture and mice brain lysates, we could show that $\alpha$-synuclein is modified in vitro and in vivo by SUMO. Both SUMO1 and SUMO2 can be conjugated to a-synuclein in vitro. For most SUMO substrates in vitro conjugation can be achieved directly by the Ubc9 E2conjugation activity. In the case of a-synuclein Ubc9 was sufficient to in vitro conjugate SUMO1 but not SUMO2 (Figure 3-1, 3-2). E3 ligase activity was needed to facilitate the covalent attachment of SUMO2 to $\alpha$-synuclein. From the tested E3 ligases Pias $x a$ facilitated SUMO1 conjugation and SUMO2 was efficiently conjugated in the presence of both RanBP2 and Pias $x$. Even though the in vivo identification of the specific SUMO E3 ligase for a-synuclein is not within the scope of this study, it remains an interesting aspect that may uncover some of the molecular players regulating $\alpha$-synuclein modification. 
We have confirmed that $\alpha$-synuclein is a SUMOylation substrate in several different cell lines (Figure 3-3). In contrast to Dorval et al., who found that $\alpha$ synuclein is mainly SUMO1 conjugated we see no preference for one of the SUMO paralogs in a-synuclein modification. Since the cell lines used for the biochemical assays were of non neuronal type it was important to show that a-synuclein is SUMOylated in cells which express the substrate also endogenously.

We employed FRET-FLIM as a complementary approach to detect SUMOylation with a spatial distribution in a neuronal cell line. Using FLIM we have demonstrated that $\alpha$-synuclein is SUMO modified in intact immortalized nigrostriatal cells (Figure 3-5). The FRET efficiency was distributed throughout the entire cell body which indicated that there is no confined cell locus enriched in SUMOylated $\alpha$ synuclein.

We went further on and characterized a-synuclein SUMOylation in vivo. Using transgenic mice overexpressing $\mathrm{His}_{6}-\mathrm{SUMO} 2$ under a neuron-specific promoter (Dr. Marilyn Tirard, unpublished data), we have shown that endogenous $\alpha$ synuclein is SUMO modified in the mouse brain (Figure 3-7). The pulldown experiments from the SUMO 2 transgenic animals revealed apart from monoSUMOylated a-synuclein also higher molecular weight a-synuclein-SUMO conjugates. In the cell culture experiments a-synuclein was found conjugated to a single SUMO molecule, which raises the hypothesis, that poly-SUMOylated $\alpha$ synuclein is more stable and/or restricted to neuronal cells. Whether the high molecular weight $\alpha$-synuclein-SUMO conjugates are result of SUMO2 chain formation or simultaneous modifications at more than one acceptor site remains to be investigated.

Using the $\mathrm{HiS}_{6}$-SUMO2 transgenic mice, we showed for the first time that a neuronal specific protein with a potential role in neurodegeneration is SUMOylated at endogenous levels in vivo.

We have shown that both SUMO1 and SUMO2 are conjugated to $\alpha$ synuclein. Whether the individual SUMO paralogs have different cellular functions is currently not known. There are, however, several indications that support the hypothesis that SUMO1 and SUMO2 can serve different roles. Among those are the low sequence homology between SUMO1 and SUMO2 and the fact that SUMO2 can build chains in vivo (Tatham et al., 2001). Additionally, the cellular pool of free SUMO2 is much bigger than of the one of SUMO1. SUMO1 and SUMO2 
conjugation seems to be differently regulated under stress conditions, like hypoxia (Yang et al., 2008). Whether $\alpha$-synuclein SUMOylation has a parolog specific effect remains unknown.

\subsection{Multiple SUMOylation sites}

Mapping the SUMOylation site is a prerequisite for elucidating the functional effects of SUMO conjugation in the context of a specific substrate. Most of the SUMOylated proteins are modified at lysine residues which are part of the consensus SUMOylation site $\psi-\mathrm{K}-\mathrm{X}-\mathrm{D} / \mathrm{E}$ (Rodriguez et al., 2001). The single E2conjugating enzyme acting in the SUMOylation machinery, Ubc9, can recognize and bind directly to consensus SUMO motifs (Sampson et al., 2001; BernierVillamor et al., 2002; Tatham et al., 2003). Among the large number of SUMO targets, some have been identified that are SUMO conjugated at non-consensus lysines. Such examples are Lys 14 of the Ubiquitin-conjugating enzyme E2-25K, where a hydrophobic residue precedes the acceptor Lys, but no Asp or Glu are present (Pichler et al., 2005). PCNA is SUMOylated at Lys 134 which belongs to the sequence T-K-E-T containing neither a large hydrophobic residue, nor an acidic one (Hoege et al., 2002). The exact mechanism how Ubc9 recognizes non-consensus SUMOylation motifs is so far not known. Most probably the facilitative function of E3 ligases is essential for their efficient conjugation.

a-Synuclein has in total fifteen lysine residues, including K96 which falls into a classical SUMO motif V-K-K-E. Lysine residue K102 is the second one estimated by SUMO site detection software (SUMO plot ${ }^{\mathrm{TM}}$, Abgent) to be conjugated with high probability. For this residue given the surrounding amino acid composition G-K-N-E the requirements for direct Ubc9 recognition are only partially fulfilled, namely the acidic glutamate residue is present but there is no bulky hydrophobic amino acid preceding the target lysine.

Using site directed mutagenesis we have shown that $\alpha$-synuclein can be modified at K96 and K102. However, they are not the only SUMO acceptor sites, since their substitution with arginine fails to completely abolish $\alpha$-synuclein SUMOylation (Figure 3-8). Constructs with alternative mutations, disrupting the negative charged amino acid downstream of K96 and K102, reproduce the same result as Lys to Arg substitutions, leading to the conclusion that those two acceptor sites are recognized as consensus SUMO motifs (Figure 3-11). 
Mass spectrometry experiments have shown that eleven out of fifteen lysines are SUMO conjugated in vitro. The SUMO conjugation sites are distributed through all 3 domains of $\alpha$-synuclein. They are found within the imperfect repeats of the $\mathrm{N}-$ terminal amphipatic region ( $\mathrm{K} 10, \mathrm{~K} 12, \mathrm{~K} 21, \mathrm{~K} 23, \mathrm{~K} 34, \mathrm{~K} 45$, and $\mathrm{K} 60$ ), the hydrophobic core region (K80) and the acidic C-terminus (K96, K102). The unusual high number of SUMOylation sites present in $\alpha$-synuclein can be due to its natively unfolded nature which leaves most of the Lys residues accessible for the SUMO conjugation machinery.

Some of the acceptor lysines are very closely situated to each other (e.g. K10 and K12: K21 and K23) and from a structural point of view their simultaneous conjugation to SUMO would be sterically hindered.

Pulldown experiments with $\alpha$-synuclein-K96R show a very slight reduction in the $\alpha$-synuclein SUMOylation level. The same was observed in the K102R $\alpha$ synuclein mutant. Simultaneous elimination of the two consensus sites K96 and K102 indeed resulted in considerably less SUMOylated a-synuclein (Figure 3-9, 311). This suggests that at least one of these consensus sites is modified at a time or in other words, an alternating SUMO conjugation to either K96 or K102 is likely to occur.

SUMO conjugation to K96 or K102 induced slight differences in SDS-PAGE mobility compared to $\alpha$-synuclein SUMOylated at $\mathrm{N}$-terminal lysine residues (Figure 3-9). This might be due to structural changes dependent on whether SUMO gets conjugated to lysines in the $\mathrm{N}$ - or $\mathrm{C}$-terminal region of $\alpha$-synuclein.

Apart from SUMOylation, lysine residues are targeted for several other posttranslational modifications - methylation, acetylation and ubiquitination, which might interfere with SUMO conjugation. a-Synuclein is ubiquitinated at various lysine residues - K21, K23, K32, and K34 - in vitro and in cell culture experiments. Filamentous $\alpha$-synuclein can also be ubiquitinated in vitro preferentially at K6, K10 and K12 (Nonaka et al., 2005). Most of these sites are also used for SUMO conjugation, but do not overlap with the major SUMOylation sites - K96 and K102. However, given the fact that almost every lysine residue in $\alpha$-synuclein can be SUMOylated, but $\alpha$-synuclein is conjugated to only one SUMO molecule at a time, direct competition between SUMO and ubiquitin in this case is rather unlikely. Moreover, mutations in the consensus SUMOylation sites did not change the ubiquitination levels of $\alpha$-synuclein. Additional evidence that SUMOylation is not 
related to stabilization of $\alpha$-synuclein came from the fact that no obvious decrease in the levels of $\alpha$-synuclein was observed upon cotransfection with the viral protein Gam1 (data not shown), which inhibits the SUMOylation machinery (Colombo et al., 2002; Boggio et al., 2004).

In line with these findings, number of detailed studies on substrates which are SUMOylated and ubiquitinated at the same lysine residue support the current notion in the field of posttranslational modifications that ubiquitination and SUMOylation occur upon specific stimuli to trigger different functional effects rather than operating on a simple competition basis. However, it remains to be shown whether SUMOylation of $\alpha$-synuclein has a conjugation-site-specific effect.

\subsection{SUMO modulates $\alpha-s y n u c l e i n$ aggregation}

Many neurodegenerative disorders including PD are characterized by insoluble proteinacious deposits.

SUMOylation can have various different consequences for the modified substrate. Modifying protein solubility and preventing aggregation is an aspect of SUMO conjugation for which very little is known. Late after SUMO's discovery, the small-Ubiquitin like modifier became popular in recombinant bacterial expression systems as a fusion tag facilitating folding and greatly enhancing solubility of the protein it is fused to (Butt et al., 2005; Marblestone et al., 2006). This raised the intriguing question whether SUMO conjugation might have a direct effect on $\alpha$ synuclein aggregation.

In the past five years SUMO conjugation has been described for several proteins prone to aggregate and present in neuronal disease related inclusions: huntingtin and ataxin-1 in Polyglutamine diseases, tau in Alzheimer's disease and a-synuclein in Parkinson's disease.

An N-terminal fusion of SUMO to the pathogenic fragment of huntingtin Httex1p has been shown to decrease Htt inclusion formation (Steffan et al., 2004). Another protein involved in polyglutamine disorders, ataxin-1, was found to be SUMO 1 conjugated. Interesting is the correlation that the aggregation prone mutant of ataxin-1 with polyglutamine expansion (82Q) was less SUMO conjugated (Riley et al., 2005). The L166P PD-related DJ1 mutant has been show to be polySUMOylated, while wild type DJ-1 is mono-SUMO conjugated. Improper 
SUMOylation is implicated in mutant DJ-1 increased inclusion formation (Shinbo et al., 2006). The relation between SUMOylation and aggregate formation in the case of tau remains unknown.

To investigate the effect of SUMOylation on $\alpha$-synuclein aggregation we used two different approaches: in vitro fibril formation and inclusion formation in a cell line based assay. Under physiological salt concentrations, non-modified wild type $\alpha$ synuclein forms mature amyloid fibrils similar to those found in synucleinopathy patients (Crowther et al., 2000; Serpell et al., 2000). Under those conditions SUMO conjugation prevented $\alpha$-synuclein fibril formation (Figure 3-18, 3-19). Interestingly, the presence of SUMO in the a-synuclein-SUMO protein mixture did not inhibit fibrillization, but resulted in formation of fibrils of smaller size. This pointed out that only SUMO which is covalently conjugated to a-synuclein is able to completely inhibit fibril formation.

Amyloid fibrils are formed from protofibrils, consisting of B-sheet structures with an orientation perpendicular to the fibril axis. Biochemical analysis has revealed a $7 \mathrm{kDa}$ fragment of a-synuclein, comprising of residues 31-109, as proteinase $\mathrm{K}$ resistant core of the amyloid fibril (Miake et al., 2002). Solid state NMR experiments with residue-specific resolution have confirmed $\alpha$-synuclein residues 30-110, as the ones comprising the amyloid fibril core (Heise et al., 2005; Vilar et al., 2008). We have shown that SUMO conjugation occurs at the single lysine residue present within the hydrophobic NAC region as well as residues very close to the aggregation promoting amino acid stretch (K34, K60, K80, K96, and K102). Therefore steric hindrance most probably accounts for impaired fibril formation of SUMOylated $\alpha$-synuclein. Once covalently attached SUMO may act as a shield for the aggregation promoting hydrophobic core of a-synuclein, thereby preventing fibrillation. Future NMR experiments on SUMOylated a-synuclein would shed light on the mechanism how SUMO conjugation impedes fibril formation. Another interesting aspect which remains to be investigated is whether SUMOylation induces folding of the natively unstructured $\alpha$-synuclein via long range tertiary interactions.

Similar inhibition effect on in vitro a-synuclein fibril formation has been observed by addition of Hsp70, Hsp40 and Hsp104 to the aggregation sample (Kong et al., 2003; Dedmon et al., 2005; Lo Bianco et al., 2008; Luk et al., 2008). For Hsp70 it has been proposed that the inhibition of fibril formation is due to its 
binding to the hydrophobic core of a-synuclein or to prefibrillar intermediates (Dedmon et al., 2005; Luk et al., 2008).

We used the PDZ based assay as a second approach to confirm the role of SUMOylation in modulating $\alpha$-synuclein aggregation in cells. Our data, obtained from the in vitro fibrillation and the PDZ cell assay, implicate a role of SUMO in increasing protein solubility. The effect of SUMO modification on another aggregopathy-related protein - Huntingtin $(\mathrm{Htt})$ is in agreement with our results. Fused to the N-terminus of $\mathrm{Htt}$, SUMO enhanced $\mathrm{Htt}$ solubility and reduced inclusion formation (Steffan et al., 2004).

Our findings together with others raise the hypothesis that SUMO, once conjugated to a-synuclein, can function as a molecular chaperone keeping aggregation prone protein fractions in solution.

\subsection{SUMOylation of $\alpha$-synuclein - role in toxicity}

One of the first indications that wild type $\alpha$-synuclein can be neurotoxic comes from familial PD patients with gene locus triplication. Failure in a-synuclein processing can be one of the reasons for dopaminergic cell death in the patients with gene dosage increases and in the idiopathic PD cases (Gwinn-Hardy et al., 2000; Singleton et al., 2003). Wild type a-synuclein has been shown to be toxic also in various fly and rodent models of PD (Park and Lee, 2006; Kahle, 2008).

We investigated the role of SUMOylation in a-synuclein induced dopaminergic cell death using the rAAV rat model of PD. Reduced a-synuclein SUMOylation by mutation of the two consensus SUMO sites exacerbated $\alpha$ synuclein toxicity and reduced the number of surviving dopaminergic cells (Figure 323, 3-24). This implicated a beneficial effect of a-synuclein SUMOylation on neuronal cell survival. The PDZ based cell culture assay confirmed an elevated toxicity of $\alpha$-synuclein lacking its two SUMO consensus sites, which also correlated with increase in aggregate formation (Figure 3-25).

Surprisingly, AAV-induced expression of $\alpha$-synuclein-KO10, lacking most SUMO conjugation sites, was less toxic than a-synuclein DM and wild type. However, it has to be considered that some of the mutated lysine residues in $\alpha$ synuclein-KO10 are also ubiquitination sites (Nonaka et al., 2005) and this mutant form is significantly less ubiquitinated compared to a-synuclein wild type and DM (Figure 3-15). The mutations in a-synuclein-DM did not interfere with the 
ubiquitination levels. The latter mutant is thus the most specific approach to study SUMOylation effects on $\alpha$-synuclein induced toxicity.

The observation that mutation of acceptor lysines common for SUMOylation and ubiquitination reduced rather than enhanced $\alpha$-synuclein pathology in the rat SNpc suggests that the toxic effect of ubiquitin is dominating over the neuroprotective action of SUMO. This is supported by a recent study of Lee et al. showing that mono- and di-ubiquitinated $\alpha$-synuclein by Siah1 are not targeted for proteasomal aggregation, but have an increased tendency to aggregate and are more cytotoxic (Lee et al., 2008). Moreover, ubiquitinated $\alpha$-synuclein has also been found in Lewy bodies of PD patients but the role of ubiquitination in synuclein pathology remains unclear (Shimura et al., 2001).

Similar to SUMOylation and ubiquitination, phosphorylation can also alter the levels of $\alpha$-synuclein toxicity. The S129A mutation is abolishing $\alpha$-synuclein's major phosphorylation site and exhibits higher neurotoxicity compared to wild type asynuclein in the rat model of PD (Gorbatyuk et al., 2008).

With the discovery that huntingtin is SUMO conjugated, SUMOylation was implicated to have a role in polyglutamine toxicity. Reduced total SUMO1 levels decreased neurodegeneration in a Drosophila model of HD. Additionally Htt97QPinduced cytotoxicity was significantly reduced by mutations destroying the SUMO conjugation sites. The authors thus raised the hypothesis that SUMOylation stabilizes Htt 97QP and thereby exacerbates its toxicity (Steffan et al., 2004). Unlike in the HD Drosophila model, overexpression of catalytically inactive SUMO E1activating enzyme and a polyQ expansion within the androgen receptor $\mathrm{N}$-terminus (ARtrQ112) intensified polyglutamine induced neurodegeneration in the SBMA (Spinal and bulbar muscular atrophy) Drosophila model (Chan et al., 2002).

The results presented in this work imply that SUMOylation of a-synuclein contributes to increased survival of dopaminergic neurons. Considering our observations and the above discussed findings of others, we can conclude that SUMOylation of neurodegeneration-inducing substrates can alter their deleterious effect in both ways - intensifying or reducing it. The effect of SUMOylation on neurodegeneration related substrates is highly target specific, in agreement with most other SUMOylated proteins. 


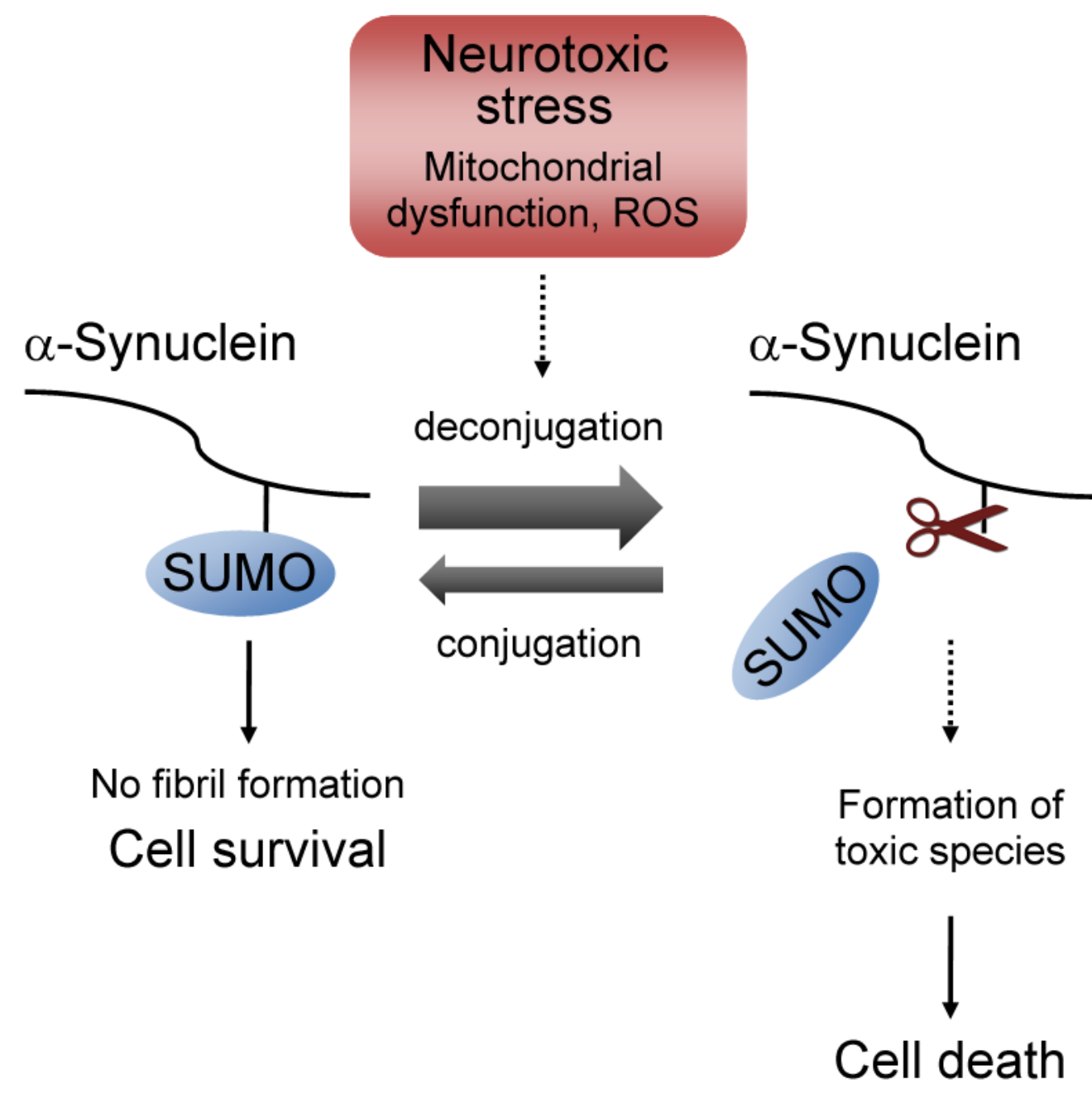

Figure 4-1. Model of the role of $\alpha$-synuclein SUMOylation in neuronal cell death. SUMO once conjugated to $\alpha$-synuclein may act as molecular chaperone, keeping aggregation prone $\alpha$-synuclein in solution and preventing aggregate formation. Neurotoxins (e.g. MPTP) and mitochondrial dysfunction lead to generation of ROS, seen in PD (Fukae et al., 2007; Zhou et al., 2008). Oxidative stress triggers SUMO deconjugation which on $\alpha$-synuclein substrate level may result in formation of toxic protein species and finally cause cell death.

In the context of $\alpha$-synuclein aggregation and toxicity, indentifying the upstream events that signal $\alpha$-synuclein-SUMO conjugation and deconjugation to would be essential for complete understanding of the role which SUMO plays for this particular target.

One hypothesis with regard to neurodegeneration would be that SUMOylation of $\alpha$-synuclein functions as a protective mechanism in the cellular response to neurotoxic factors. Dynamic SUMO modification may serve as a means to keep aggregation prone $\alpha$-synuclein fractions in solution and prevent formation of toxic intracellular inclusions. Additionally, SUMOylation may be required to modulate $\alpha$-synuclein interactions with other proteins and lipid vesicles, thereby 
regulating its potential physiological functions. An increase in production of ROS due to mitochondrial dysfunction has been observed in animal models and implicated in the pathology of PD (Fukae et al., 2007; Zhou et al., 2008). Neurotoxins, like MPTP lead to redistribution of vesicular dopamine to the cytosol where dopamine auto-oxidizes, thereby generating free radicals (Jackson-Lewis and Smeyne, 2005). Oxidative stress has been shown to decrease SUMO conjugation by inhibition of the SUMOylation enzymes (Bossis and Melchior, 2006). At the substrate level of $\alpha$-synuclein this could possibly lead to generation of toxic $\alpha$ synuclein species and ultimately to cell death (Figure 4-1). Thus SUMOylation by linking oxidative stress and $\alpha$-synuclein-induced toxicity might be part of the plethora of factors involved in PD pathology. 


\section{Summary}

SUMOylation is a highly dynamic posttranslational modification in which covalent attachment of the small ubiquitin-like modifier protein (SUMO) is modulating different substrate specific properties - activity, structural conformation or localization. Posttranslational modifications including SUMOylation are essential parts of signalling pathways in the central nervous system (CNS). Their tight control is of crucial importance for maintaining neuronal cell function, connectivity and viability.

A few neuronal specific SUMO substrates have been discovered in the last years but very little is known about the role of SUMOylation in neuronal cell death and survival. We show that $\alpha$-synuclein is SUMO1 and SUMO2 conjugated in vitro and in different cell lines. Moreover, we found SUMOylated a-synuclein in the mouse brain in vivo.

Mass spectrometry analysis of in vitro SUMOylated a-synuclein revealed eleven lysine residues as SUMO conjugation sites. More detailed mutagenesis experiments identified K96 and K102 as the major "alternative" SUMOylation site in a-synuclein.

This work reports that covalent attachment of SUMO is regulating $\alpha$-synuclein induced toxicity and aggregation. SUMO conjugation to $\alpha$-synuclein led to inhibition of amyloid fibril formation in vitro. Mutations in the two SUMO consensus lysine residues exacerbated $\alpha$-synuclein induced cytotoxicity in the rat Parkinson's disease (PD) model. This result demonstrates that endogenous SUMOylation of $\alpha$-synuclein at the consensus K96/K102 SUMO acceptor site(s) greatly reduces its neurotoxicity on dopaminergic SNpc neurons in vivo.

In agreement with the results obtained in the in vivo PD model, in vitro assay in HEK cells showed that reduction in a-synuclein SUMOylation levels results in increased aggregate formation and cell death rate.

This work presents SUMOylation as a novel factor modulating the most prominent PD-related characteristics of $\alpha$-synuclein, neurotoxicity and fibril formation, and reveals a potential disease-related function of SUMOylation in the CNS. 


\section{Appendix 1.}

\section{The Cyclin-dependent kinase 5 regulator p35 is a novel SUMOylation target}

Cyclin-dependent kinase 5 (Cdk5) belongs to the family of proline-directed Ser/Thr kinases. Cdk5 is evolutionary conserved across eukaryotic species and ubiquitously expressed. Its activity, however, was detected primarily in the nervous system due to the neuron specific localization of the two non-cyclin Cdk5 activators - p35 (NCK5a, neuronal Cdk5 activator) and p39 (NCK5ai, neuronal Cdk5 activator isoform) (Tsai et al., 1994). In contrast to other Cdks, Cdk5 has a minor role in cell cycle signaling (Zhang et al., 2008b). In the developing brain Cdk5 is required for proper neuronal migration, axonal growth and synapse maturation (Dhavan and Tsai, 2001). Interestingly, misregulation of Cdk5 in post-mitotic neurons occurs in several neurodegenerative disorders, including Alzheimer's disease, Parkinson's disease and amyotrophic lateral sclerosis. Disturbed calcium homeostasis in hypoxic or other stress conditions induces calpain-mediated proteolytic cleavage of p35 and formation of p25, an N-terminally truncated form of p35. Unlike p35, p25 is not readily degraded and binding of p25 to Cdk5 leads to constitutive activity of Cdk5, changes of subcellular distribution and altered substrate specificity, which finally results in neuronal cell death (Patrick et al., 1999). Since processing and subcellular targeting of p35 is a crucial switch for the neurotoxic function of Cdk5, posttranslational modifications might be important in this process. Therefore we asked if SUMOylation has a role in the modulation of p35/CDK5.

We first tested if p35/p25 and Cdk5 are targets for SUMO conjugation. Using recombinant SUMOylation enzymes and p35 or p25 purified with Cdk5 we found out that SUMO1 is conjugated to both p35 and p25, but not Cdk5. The p35/Cdk5 complex in the in vitro reaction mixture is catalytically active and in the presence of energy source Cdk5 phosphorylates the entire pool of p35. The SUMO modified p35 fraction is presumably also phosphorylated. In vitro SUMOylation of p35/p25 requires the presence of E3 ligase. Two among the tested E3 ligases, the catalytic fragment of RanBP2 and the full length Pias ${ }_{x \alpha}$ facilitated p35 and p25 modification by SUMO1. RanBP2 is associated with the nuclear pore complex and PIAS family members have predominantly nuclear localization, which raises the hypothesis that SUMO1 conjugation to p35/p25 might be involved in nuclear transport and 
localization. Moreover, Cdk5 activity has been shown both in the cytosol and the nucleus (Ino and Chiba, 1996). p35 can associate with the plasma membrane via its $\mathrm{N}$-terminal myristoylation. However, a fraction of p35 is imported into the nucleus, which involves importin $\beta / 5 / 7$ (Fu et al., 2006).

To examine if SUMO has an effect on p35 cellular distribution we cotransfected GFP-tagged p35 and SUMO or empty vector in neuronal CSM14.1 cells and evaluated GFP fluorescence intensity in the nucleus versus cytoplasm. Cotransfection of SUMO led to increase in the nuclear GFP fluorescence compared to empty vector controls, which implies a role of SUMO in p35 nuclear localization. To confirm this observation we are planning to employ p35 mutants, which are deficient in SUMO conjugation as negative controls.

We did not observe SUMO2 conjugation neither to p35 nor p25 in vitro. However, using $\mathrm{Ni}^{2+}$-NTA chromatography we could show that p35 as well as p25 are conjugated by both SUMO1 and SUMO2 isoforms in two different cell lines (HeLa and HEK 293). This suggests involvement of different E3 ligase from those tested in vitro in the p35/p25 SUMO2 conjugation in vivo. Pulldown experiments with SUMO have shown p35 conjugated to a single SUMO1 molecule as well as two additional higher molecular weight species. The latter might result from two and more SUMO1 molecules conjugated at different acceptor lysines, since SUMO1 was proven not to form chains in vivo. SUMO2 modification of p35 led to the appearance of significantly more species of higher molecular weight, which could involve SUMO2 chain formation in addition to SUMO conjugation at different sites.

To determine whether association with Cdk5 is required for p35 SUMOylation, we used two p35 mutants, p35 (L151, 152N) and p35 (D288A, L289A), which are devoid of Cdk5-binding activity (Tang et al., 1997). Interestingly, p35 (L151, 152N) was SUMOylated to the same extent as wild type p35, while p35 (D288A, L289A) SUMOylation was strongly reduced. This points out that the formation of p35-CDK5 complex is not necessary for SUMOylation. More likely the mutations in the second mutant - D288A and especially L289A interfere with SUMOylation directly. This is supported by the evidence that L289 is part of the single consensus SUMO motif (L-K-N-E) in the amino acid sequence of p35. Additionally, mutation of K290 led to a reduction in the p35 SUMO conjugation. K290, however, is not the single SUMO conjugation site in p35 since it did not completely abolish SUMOylation. This is in agreement with the observed high 
molecular weight p35-SUMO1 conjugates. Ongoing mutagenesis analysis aims to identify the major SUMO conjugation sites and generation of a SUMOylation deficient p35 mutant for further functional analysis.

In a self-attenuation mechanism similar for all Cyclin-dependent kinases, CDK5 phosphorylates its activator p35 which induces p35 ubiquitination and proteasomal degradation (Patrick et al., 1998). Therefore, we asked if analogous to ubiquitination, SUMOylation of p35 is regulated by phosphorylation. Wild type or phosphorylation-deficient p35 (S8A, T138A, S170A, T197A) mutant was cotransfected together $\mathrm{His}_{6}-\mathrm{SUMO} 2$ and SUMOylated proteins were isolated via $\mathrm{Ni}^{2+}$-NTA chromatography. Indeed, SUMO2 conjugation was decreased in the case of phosphorylation-deficient p35. This indicates that similar to ubiqutitnation phosphorylation has a positive regulatory effect on SUMOylation. Whether SUMO stabilizes p35 and thus has an opposing role to ubiquitination remains to be examined.

In summary, we have identified the neuronal specific regulator of Cdk5 - p35 as a SUMO target in vitro and in different cell lines. The truncated form p25 is SUMO modified as well. p35 has multiple SUMOylation sites, one of which is K290. Our experiments show that phosphorylation of p35 stimulates its SUMOylation. Moreover, SUMO might be involved in p35 nuclear translocation.

In future experiments with SUMOylation deficient p35 mutants we aim to delineate the ways SUMOylation, as a novel posttranslational modification of p35, regulates p35/Cdk5 function in the developing neuron and in neurodegeneration. We plan to investigate the role of SUMOylation in p35 association to Cdk5 and the subsequent kinase activation. We would like to test if SUMO conjugation has an impact on neurite outgrowth of primary cortical neurons as well as if SUMOylation modulates the neurotoxic calpain-induced cleavage of p35. 


\section{References}

Abeliovich A, Schmitz Y, Farinas I, Choi-Lundberg D, Ho WH, Castillo PE, Shinsky N, Verdugo JM, Armanini M, Ryan A, Hynes M, Phillips H, Sulzer D, Rosenthal A (2000) Mice lacking alpha-synuclein display functional deficits in the nigrostriatal dopamine system. Neuron 25:239-252.

Alkuraya FS, Saadi I, Lund JJ, Turbe-Doan A, Morton CC, Maas RL (2006) SUMO1 haploinsufficiency leads to cleft lip and palate. Science 313:1751.

Andrews EA, Palecek J, Sergeant J, Taylor E, Lehmann AR, Watts FZ (2005) Nse2, a component of the Smc5-6 complex, is a SUMO ligase required for the response to DNA damage. Mol Cell Biol 25:185-196.

Azeredo da Silveira S, Schneider BL, Cifuentes-Diaz C, Sage D, Abbas-Terki T, Iwatsubo T, Unser M, Aebischer P (2009) Phosphorylation does not prompt, nor prevent, the formation of alpha-synuclein toxic species in a rat model of Parkinson's disease. Hum Mol Genet 18:872-887.

Baba D, Maita N, Jee JG, Uchimura Y, Saitoh H, Sugasawa K, Hanaoka F, Tochio H, Hiroaki $H$, Shirakawa M (2005) Crystal structure of thymine DNA glycosylase conjugated to SUMO-1. Nature 435:979-982.

Baba D, Maita N, Jee JG, Uchimura Y, Saitoh H, Sugasawa K, Hanaoka F, Tochio H, Hiroaki H, Shirakawa M (2006) Crystal structure of SUMO-3-modified thymine-DNA glycosylase. J Mol Biol 359:137-147.

Bartlett JS, Samulski RJ, McCown TJ (1998) Selective and rapid uptake of adenoassociated virus type 2 in brain. Hum Gene Ther 9:1181-1186.

Bayer P, Arndt A, Metzger S, Mahajan R, Melchior F, Jaenicke R, Becker J (1998) Structure determination of the small ubiquitin-related modifier SUMO-1. J Mol Biol 280:275-286.

Bennett MC, Bishop JF, Leng Y, Chock PB, Chase TN, Mouradian MM (1999) Degradation of alpha-synuclein by proteasome. J Biol Chem 274:33855-33858.

Benson MD, Li QJ, Kieckhafer K, Dudek D, Whorton MR, Sunahara RK, Iniguez-Lluhi JA, Martens JR (2007) SUMO modification regulates inactivation of the voltage-gated potassium channel Kv1.5. Proc Natl Acad Sci U S A 104:1805-1810.

Bernier-Villamor V, Sampson DA, Matunis MJ, Lima CD (2002) Structural basis for E2mediated SUMO conjugation revealed by a complex between ubiquitin-conjugating enzyme Ubc9 and RanGAP1. Cell 108:345-356.

Best JL, Ganiatsas S, Agarwal S, Changou A, Salomoni P, Shirihai O, Meluh PB, Pandolfi PP, Zon LI (2002) SUMO-1 protease-1 regulates gene transcription through PML. Mol Cell 10:843-855.

Boggio R, Colombo R, Hay RT, Draetta GF, Chiocca S (2004) A mechanism for inhibiting the SUMO pathway. Mol Cell 16:549-561.

Bohren KM, Nadkarni V, Song JH, Gabbay KH, Owerbach D (2004) A M55V polymorphism in a novel SUMO gene (SUMO-4) differentially activates heat shock transcription factors and is associated with susceptibility to type I diabetes mellitus. J Biol Chem 279:27233-27238.

Bossis G, Melchior F (2006) Regulation of SUMOylation by reversible oxidation of SUMO conjugating enzymes. Mol Cell 21:349-357.

Butt TR, Edavettal SC, Hall JP, Mattern MR (2005) SUMO fusion technology for difficult-toexpress proteins. Protein Expr Purif 43:1-9.

Cabin DE, Shimazu K, Murphy D, Cole NB, Gottschalk W, Mcllwain KL, Orrison B, Chen A, Ellis CE, Paylor R, Lu B, Nussbaum RL (2002) Synaptic vesicle depletion correlates with attenuated synaptic responses to prolonged repetitive stimulation in mice lacking alpha-synuclein. J Neurosci 22:8797-8807.

Carbia-Nagashima A, Gerez J, Perez-Castro C, Paez-Pereda M, Silberstein S, Stalla GK, Holsboer F, Arzt E (2007) RSUME, a small RWD-containing protein, enhances SUMO conjugation and stabilizes HIF-1alpha during hypoxia. Cell 131:309-323. 
Chan HY, Warrick JM, Andriola I, Merry D, Bonini NM (2002) Genetic modulation of polyglutamine toxicity by protein conjugation pathways in Drosophila. Hum Mol Genet 11:2895-2904.

Chandra S, Gallardo G, Fernandez-Chacon R, Schluter OM, Sudhof TC (2005) Alphasynuclein cooperates with CSPalpha in preventing neurodegeneration. Cell 123:383-396.

Chen L, Feany MB (2005) Alpha-synuclein phosphorylation controls neurotoxicity and inclusion formation in a Drosophila model of Parkinson disease. Nat Neurosci 8:657663.

Cheng TS, Chang LK, Howng SL, Lu PJ, Lee CI, Hong YR (2006) SUMO-1 modification of centrosomal protein hNinein promotes hNinein nuclear localization. Life Sci 78:1114-1120.

Colombo R, Boggio R, Seiser C, Draetta GF, Chiocca S (2002) The adenovirus protein Gam1 interferes with sumoylation of histone deacetylase 1. EMBO Rep 3:10621068.

Conway KA, Rochet JC, Bieganski RM, Lansbury PT, Jr. (2001) Kinetic stabilization of the alpha-synuclein protofibril by a dopamine-alpha-synuclein adduct. Science 294:1346-1349.

Cookson MR (2005) The biochemistry of Parkinson's disease. Annu Rev Biochem 74:2952.

Cooper AA, Gitler AD, Cashikar A, Haynes CM, Hill KJ, Bhullar B, Liu K, Xu K, Strathearn KE, Liu F, Cao S, Caldwell KA, Caldwell GA, Marsischky G, Kolodner RD, Labaer J, Rochet JC, Bonini NM, Lindquist S (2006) Alpha-synuclein blocks ER-Golgi traffic and Rab1 rescues neuron loss in Parkinson's models. Science 313:324-328.

Crowther RA, Daniel SE, Goedert M (2000) Characterisation of isolated alpha-synuclein filaments from substantia nigra of Parkinson's disease brain. Neurosci Lett 292:128130.

De Strooper B, Annaert W (2000) Proteolytic processing and cell biological functions of the amyloid precursor protein. J Cell Sci 113 ( Pt 11):1857-1870.

Dedmon MM, Christodoulou J, Wilson MR, Dobson CM (2005) Heat shock protein 70 inhibits alpha-synuclein fibril formation via preferential binding to prefibrillar species. J Biol Chem 280:14733-14740.

Desterro JM, Thomson J, Hay RT (1997) Ubch9 conjugates SUMO but not ubiquitin. FEBS Lett 417:297-300.

Desterro JM, Rodriguez MS, Kemp GD, Hay RT (1999) Identification of the enzyme required for activation of the small ubiquitin-like protein SUMO-1. J Biol Chem 274:10618-10624.

Dhavan R, Tsai LH (2001) A decade of CDK5. Nat Rev Mol Cell Biol 2:749-759.

Di Bacco A, Ouyang J, Lee HY, Catic A, Ploegh H, Gill G (2006) The SUMO-specific protease SENP5 is required for cell division. Mol Cell Biol 26:4489-4498.

Dohm CP, Kermer P, Bahr M (2008) Aggregopathy in neurodegenerative diseases: mechanisms and therapeutic implication. Neurodegener Dis 5:321-338.

Dorval V, Fraser PE (2006) Small ubiquitin-like modifier (SUMO) modification of natively unfolded proteins tau and alpha-synuclein. J Biol Chem 281:9919-9924.

Dorval V, Mazzella MJ, Mathews PM, Hay RT, Fraser PE (2007) Modulation of Abeta generation by small ubiquitin-like modifiers does not require conjugation to target proteins. Biochem J 404:309-316.

Ellis CE, Schwartzberg PL, Grider TL, Fink DW, Nussbaum RL (2001) alpha-synuclein is phosphorylated by members of the Src family of protein-tyrosine kinases. J Biol Chem 276:3879-3884.

Endter C, Kzhyshkowska J, Stauber R, Dobner T (2001) SUMO-1 modification required for transformation by adenovirus type 5 early region 1B 55-kDa oncoprotein. Proc Natl Acad Sci U S A 98:11312-11317.

Feany MB, Bender WW (2000) A Drosophila model of Parkinson's disease. Nature 404:394-398. 
Forster T (1948) Zwischenmolekulare Energiewanderung und Fluoreszenz. Annalen der Physik 437:55-75.

Fu X, Choi YK, Qu D, Yu Y, Cheung NS, Qi RZ (2006) Identification of nuclear import mechanisms for the neuronal Cdk5 activator. J Biol Chem 281:39014-39021.

Fujiwara H, Hasegawa M, Dohmae N, Kawashima A, Masliah E, Goldberg MS, Shen J, Takio K, Iwatsubo T (2002) alpha-Synuclein is phosphorylated in synucleinopathy lesions. Nat Cell Biol 4:160-164.

Fukae J, Mizuno Y, Hattori N (2007) Mitochondrial dysfunction in Parkinson's disease. Mitochondrion 7:58-62.

Geiss-Friedlander R, Melchior F (2007) Concepts in sumoylation: a decade on. Nat Rev Mol Cell Biol 8:947-956.

Giasson BI, Uryu K, Trojanowski JQ, Lee VM (1999) Mutant and wild type human alphasynucleins assemble into elongated filaments with distinct morphologies in vitro. $\mathrm{J}$ Biol Chem 274:7619-7622.

Giasson BI, Duda JE, Murray IV, Chen Q, Souza JM, Hurtig HI, Ischiropoulos H, Trojanowski JQ, Lee VM (2000) Oxidative damage linked to neurodegeneration by selective alpha-synuclein nitration in synucleinopathy lesions. Science 290:985-989.

Gong L, Yeh ET (2006) Characterization of a family of nucleolar SUMO-specific proteases with preference for SUMO-2 or SUMO-3. J Biol Chem 281:15869-15877.

Gong L, Li B, Millas S, Yeh ET (1999) Molecular cloning and characterization of human AOS1 and UBA2, components of the sentrin-activating enzyme complex. FEBS Lett 448:185-189.

Gong L, Millas S, Maul GG, Yeh ET (2000) Differential regulation of sentrinized proteins by a novel sentrin-specific protease. J Biol Chem 275:3355-3359.

Goodson ML, Hong Y, Rogers R, Matunis MJ, Park-Sarge OK, Sarge KD (2001) Sumo-1 modification regulates the DNA binding activity of heat shock transcription factor 2 , a promyelocytic leukemia nuclear body associated transcription factor. J Biol Chem 276:18513-18518.

Gorbatyuk OS, Li S, Sullivan LF, Chen W, Kondrikova G, Manfredsson FP, Mandel RJ, Muzyczka N (2008) The phosphorylation state of Ser-129 in human alpha-synuclein determines neurodegeneration in a rat model of Parkinson disease. Proc Natl Acad Sci U S A 105:763-768.

Grimm D, Kern A, Rittner K, Kleinschmidt JA (1998) Novel tools for production and purification of recombinant adenoassociated virus vectors. Hum Gene Ther 9:27452760.

Guo D, Li M, Zhang Y, Yang P, Eckenrode S, Hopkins D, Zheng W, Purohit S, Podolsky $\mathrm{RH}$, Muir A, Wang J, Dong Z, Brusko T, Atkinson M, Pozzilli P, Zeidler A, Raffel LJ, Jacob CO, Park Y, Serrano-Rios M, Larrad MT, Zhang Z, Garchon HJ, Bach JF, Rotter JI, She JX, Wang CY (2004) A functional variant of SUMO4, a new I kappa B alpha modifier, is associated with type 1 diabetes. Nat Genet 36:837-841.

Gwinn-Hardy KA, Crook R, Lincoln S, Adler CH, Caviness JN, Hardy J, Farrer M (2000) A kindred with Parkinson's disease not showing genetic linkage to established loci. Neurology 54:504-507.

Haas SJ, Wree A (2002) Dopaminergic differentiation of the Nurr1-expressing immortalized mesencephalic cell line CSM14.1 in vitro. J Anat 201:61-69.

Hang J, Dasso M (2002) Association of the human SUMO-1 protease SENP2 with the nuclear pore. J Biol Chem 277:19961-19966.

Hasegawa M, Fujiwara H, Nonaka T, Wakabayashi K, Takahashi H, Lee VM, Trojanowski JQ, Mann D, Iwatsubo T (2002) Phosphorylated alpha-synuclein is ubiquitinated in alpha-synucleinopathy lesions. J Biol Chem 277:49071-49076.

Hashimoto M, Rockenstein E, Mante M, Mallory M, Masliah E (2001) beta-Synuclein inhibits alpha-synuclein aggregation: a possible role as an anti-parkinsonian factor. Neuron 32:213-223.

Heise H, Hoyer W, Becker S, Andronesi OC, Riedel D, Baldus M (2005) Molecular-level secondary structure, polymorphism, and dynamics of full-length alpha-synuclein fibrils studied by solid-state NMR. Proc Natl Acad Sci U S A 102:15871-15876. 
Hietakangas V, Anckar J, Blomster HA, Fujimoto M, Palvimo JJ, Nakai A, Sistonen L (2006) PDSM, a motif for phosphorylation-dependent SUMO modification. Proc Natl Acad Sci U S A 103:45-50.

Hietakangas V, Ahlskog JK, Jakobsson AM, Hellesuo M, Sahlberg NM, Holmberg Cl, Mikhailov A, Palvimo JJ, Pirkkala L, Sistonen L (2003) Phosphorylation of serine 303 is a prerequisite for the stress-inducible SUMO modification of heat shock factor 1. Mol Cell Biol 23:2953-2968.

Hoege C, Pfander B, Moldovan GL, Pyrowolakis G, Jentsch S (2002) RAD6-dependent DNA repair is linked to modification of PCNA by ubiquitin and SUMO. Nature 419:135-141.

Hong Y, Rogers R, Matunis MJ, Mayhew CN, Goodson ML, Park-Sarge OK, Sarge KD (2001) Regulation of heat shock transcription factor 1 by stress-induced SUMO-1 modification. J Biol Chem 276:40263-40267.

Ino H, Chiba T (1996) Intracellular localization of cyclin-dependent kinase 5 (CDK5) in mouse neuron: CDK5 is located in both nucleus and cytoplasm. Brain Res 732:179185.

Ivankovic-Dikic I, Gronroos E, Blaukat A, Barth BU, Dikic I (2000) Pyk2 and FAK regulate neurite outgrowth induced by growth factors and integrins. Nat Cell Biol 2:574-581.

Jackson-Lewis V, Smeyne RJ (2005) MPTP and SNpc DA neuronal vulnerability: role of dopamine, superoxide and nitric oxide in neurotoxicity. Minireview. Neurotox Res 7:193-202.

Jaffray EG, Hay RT (2006) Detection of modification by ubiquitin-like proteins. Methods 38:35-38.

Johnson ES, Blobel G (1997) Ubc9p is the conjugating enzyme for the ubiquitin-like protein Smt3p. J Biol Chem 272:26799-26802.

Johnson ES, Gupta AA (2001) An E3-like factor that promotes SUMO conjugation to the yeast septins. Cell 106:735-744.

Kadare G, Toutant M, Formstecher E, Corvol JC, Carnaud M, Boutterin MC, Girault JA (2003) PIAS1-mediated sumoylation of focal adhesion kinase activates its autophosphorylation. J Biol Chem 278:47434-47440.

Kagey MH, Melhuish TA, Wotton D (2003) The polycomb protein Pc2 is a SUMO E3. Cell 113:127-137.

Kahle PJ (2008) alpha-Synucleinopathy models and human neuropathology: similarities and differences. Acta Neuropathol 115:87-95.

Kahyo T, Nishida T, Yasuda H (2001) Involvement of PIAS1 in the sumoylation of tumor suppressor p53. Mol Cell 8:713-718.

Kamitani T, Kito K, Nguyen HP, Wada H, Fukuda-Kamitani T, Yeh ET (1998) Identification of three major sentrinization sites in PML. J Biol Chem 273:26675-26682.

Kim KI, Baek SH, Jeon YJ, Nishimori S, Suzuki T, Uchida S, Shimbara N, Saitoh H, Tanaka $\mathrm{K}$, Chung $\mathrm{CH}$ (2000) A new SUMO-1-specific protease, SUSP1, that is highly expressed in reproductive organs. J Biol Chem 275:14102-14106.

Kirik D, Rosenblad C, Burger C, Lundberg C, Johansen TE, Muzyczka N, Mandel RJ, Bjorklund A (2002) Parkinson-like neurodegeneration induced by targeted overexpression of alpha-synuclein in the nigrostriatal system. J Neurosci 22:27802791.

Kirsh O, Seeler JS, Pichler A, Gast A, Muller S, Miska E, Mathieu M, Harel-Bellan A, Kouzarides T, Melchior F, Dejean A (2002) The SUMO E3 ligase RanBP2 promotes modification of the HDAC4 deacetylase. Embo J 21:2682-2691.

Knipscheer P, Flotho A, Klug H, Olsen JV, van Dijk WJ, Fish A, Johnson ES, Mann M, Sixma TK, Pichler A (2008) Ubc9 sumoylation regulates SUMO target discrimination. Mol Cell 31:371-382.

Kong B, Chae YK, Lee K (2003) Regulation of in vitro fibril formation of synuclein mutant proteins by Hsp104p. Protein Pept Lett 10:491-495.

Kozak M (1987) At least six nucleotides preceding the AUG initiator codon enhance translation in mammalian cells. J Mol Biol 196:947-950. 
Kruger R, Kuhn W, Muller T, Woitalla D, Graeber M, Kosel S, Przuntek H, Epplen JT, Schols L, Riess O (1998) Ala30Pro mutation in the gene encoding alpha-synuclein in Parkinson's disease. Nat Genet 18:106-108.

Kugler S, Lingor P, Scholl U, Zolotukhin S, Bahr M (2003) Differential transgene expression in brain cells in vivo and in vitro from AAV-2 vectors with small transcriptional control units. Virology 311:89-95.

Kugler S, Meyn L, Holzmuller H, Gerhardt E, Isenmann S, Schulz JB, Bahr M (2001) Neuron-specific expression of therapeutic proteins: evaluation of different cellular promoters in recombinant adenoviral vectors. Mol Cell Neurosci 17:78-96.

La Spada AR, Wilson EM, Lubahn DB, Harding AE, Fischbeck KH (1991) Androgen receptor gene mutations in X-linked spinal and bulbar muscular atrophy. Nature 352:77-79.

Lapenta V, Chiurazzi P, van der Spek P, Pizzuti A, Hanaoka F, Brahe C (1997) SMT3A, a human homologue of the S. cerevisiae SMT3 gene, maps to chromosome 21qter and defines a novel gene family. Genomics 40:362-366.

Lee GW, Melchior F, Matunis MJ, Mahajan R, Tian Q, Anderson P (1998) Modification of Ran GTPase-activating protein by the small ubiquitin-related modifier SUMO-1 requires Ubc9, an E2-type ubiquitin-conjugating enzyme homologue. J Biol Chem 273:6503-6507.

Lee JT, Wheeler TC, Li L, Chin LS (2008) Ubiquitination of alpha-synuclein by Siah-1 promotes alpha-synuclein aggregation and apoptotic cell death. Hum Mol Genet 17:906-917.

Lee PS, Chang C, Liu D, Derynck R (2003) Sumoylation of Smad4, the common Smad mediator of transforming growth factor-beta family signaling. J Biol Chem 278:27853-27863.

Lee VM, Trojanowski JQ (2006) Mechanisms of Parkinson's disease linked to pathological alpha-synuclein: new targets for drug discovery. Neuron 52:33-38.

Li Y, Wang H, Wang S, Quon D, Liu YW, Cordell B (2003) Positive and negative regulation of APP amyloidogenesis by sumoylation. Proc Natl Acad Sci U S A 100:259-264.

Liani E, Eyal A, Avraham E, Shemer R, Szargel R, Berg D, Bornemann A, Riess O, Ross CA, Rott R, Engelender S (2004) Ubiquitylation of synphilin-1 and alpha-synuclein by SIAH and its presence in cellular inclusions and Lewy bodies imply a role in Parkinson's disease. Proc Natl Acad Sci U S A 101:5500-5505.

Lieberman AP, Robitaille Y, Trojanowski JQ, Dickson DW, Fischbeck KH (1998) Polyglutamine-containing aggregates in neuronal intranuclear inclusion disease. Lancet 351:884.

Lin JY, Ohshima T, Shimotohno K (2004) Association of Ubc9, an E2 ligase for SUMO conjugation, with $\mathrm{p} 53$ is regulated by phosphorylation of p53. FEBS Lett 573:15-18.

Liu C, Fei E, Jia N, Wang H, Tao R, Iwata A, Nukina N, Zhou J, Wang G (2007) Assembly of lysine 63-linked ubiquitin conjugates by phosphorylated alpha-synuclein implies Lewy body biogenesis. J Biol Chem 282:14558-14566.

Lo Bianco C, Shorter J, Regulier E, Lashuel H, Iwatsubo T, Lindquist S, Aebischer P (2008) Hsp104 antagonizes alpha-synuclein aggregation and reduces dopaminergic degeneration in a rat model of Parkinson disease. J Clin Invest 118:3087-3097.

Lowe J, Blanchard A, Morrell K, Lennox G, Reynolds L, Billett M, Landon M, Mayer RJ (1988) Ubiquitin is a common factor in intermediate filament inclusion bodies of diverse type in man, including those of Parkinson's disease, Pick's disease, and Alzheimer's disease, as well as Rosenthal fibres in cerebellar astrocytomas, cytoplasmic bodies in muscle, and mallory bodies in alcoholic liver disease. J Pathol 155:9-15.

Luk KC, Mills IP, Trojanowski JQ, Lee VM (2008) Interactions between Hsp70 and the hydrophobic core of alpha-synuclein inhibit fibril assembly. Biochemistry 47:1261412625.

Macauley MS, Errington WJ, Scharpf M, Mackereth CD, Blaszczak AG, Graves BJ, Mclntosh LP (2006) Beads-on-a-string, characterization of ETS-1 sumoylated within its flexible N-terminal sequence. J Biol Chem 281:4164-4172. 
Mahajan R, Gerace L, Melchior F (1998) Molecular characterization of the SUMO-1 modification of RanGAP1 and its role in nuclear envelope association. J Cell Biol 140:259-270.

Mahajan R, Delphin C, Guan T, Gerace L, Melchior F (1997) A small ubiquitin-related polypeptide involved in targeting RanGAP1 to nuclear pore complex protein RanBP2. Cell 88:97-107.

Malakhov MP, Mattern MR, Malakhova OA, Drinker M, Weeks SD, Butt TR (2004) SUMO fusions and SUMO-specific protease for efficient expression and purification of proteins. J Struct Funct Genomics 5:75-86.

Marblestone JG, Edavettal SC, Lim Y, Lim P, Zuo X, Butt TR (2006) Comparison of SUMO fusion technology with traditional gene fusion systems: enhanced expression and solubility with SUMO. Protein Sci 15:182-189.

Martin S, Nishimune A, Mellor JR, Henley JM (2007a) SUMOylation regulates kainatereceptor-mediated synaptic transmission. Nature 447:321-325.

Martin S, Wilkinson KA, Nishimune A, Henley JM (2007b) Emerging extranuclear roles of protein SUMOylation in neuronal function and dysfunction. Nat Rev Neurosci 8:948959.

Masliah E, Rockenstein E, Veinbergs I, Mallory M, Hashimoto M, Takeda A, Sagara Y, Sisk A, Mucke L (2000) Dopaminergic loss and inclusion body formation in alphasynuclein mice: implications for neurodegenerative disorders. Science 287:12651269.

Matunis MJ, Coutavas E, Blobel G (1996) A novel ubiquitin-like modification modulates the partitioning of the Ran-GTPase-activating protein RanGAP1 between the cytosol and the nuclear pore complex. J Cell Biol 135:1457-1470.

McLean PJ, Kawamata H, Hyman BT (2001) Alpha-synuclein-enhanced green fluorescent protein fusion proteins form proteasome sensitive inclusions in primary neurons. Neuroscience 104:901-912.

Melchior F (2000) SUMO--nonclassical ubiquitin. Annu Rev Cell Dev Biol 16:591-626.

Miake H, Mizusawa H, Iwatsubo T, Hasegawa M (2002) Biochemical characterization of the core structure of alpha-synuclein filaments. J Biol Chem 277:19213-19219.

Nacerddine K, Lehembre F, Bhaumik M, Artus J, Cohen-Tannoudji M, Babinet C, Pandolfi PP, Dejean A (2005) The SUMO pathway is essential for nuclear integrity and chromosome segregation in mice. Dev Cell 9:769-779.

Nakagawa K, Yokosawa H (2002) PIAS3 induces SUMO-1 modification and transcriptional repression of IRF-1. FEBS Lett 530:204-208.

Nakamura T, Yamashita H, Nagano Y, Takahashi T, Avraham S, Avraham H, Matsumoto M, Nakamura S (2002) Activation of Pyk2/RAFTK induces tyrosine phosphorylation of alpha-synuclein via Src-family kinases. FEBS Lett 521:190-194.

Neuhoff V, Arold N, Taube D, Ehrhardt W (1988) Improved staining of proteins in polyacrylamide gels including isoelectric focusing gels with clear background at nanogram sensitivity using Coomassie Brilliant Blue G-250 and R-250. Electrophoresis 9:255-262.

Nishida T, Yasuda H (2002) PIAS1 and PIASxalpha function as SUMO-E3 ligases toward androgen receptor and repress androgen receptor-dependent transcription. J Biol Chem 277:41311-41317.

Nishida T, Tanaka H, Yasuda H (2000) A novel mammalian Smt3-specific isopeptidase 1 (SMT3IP1) localized in the nucleolus at interphase. Eur J Biochem 267:6423-6427.

Nonaka T, Iwatsubo T, Hasegawa M (2005) Ubiquitination of alpha-synuclein. Biochemistry 44:361-368.

Norris EH, Giasson BI, Ischiropoulos H, Lee VM (2003) Effects of oxidative and nitrative challenges on alpha-synuclein fibrillogenesis involve distinct mechanisms of protein modifications. J Biol Chem 278:27230-27240.

Okochi M, Walter J, Koyama A, Nakajo S, Baba M, Iwatsubo T, Meijer L, Kahle PJ, Haass C (2000) Constitutive phosphorylation of the Parkinson's disease associated alphasynuclein. J Biol Chem 275:390-397. 
Okuma T, Honda R, Ichikawa G, Tsumagari N, Yasuda H (1999) In vitro SUMO-1 modification requires two enzymatic steps, E1 and E2. Biochem Biophys Res Commun 254:693-698.

Opazo F, Krenz A, Heermann S, Schulz JB, Falkenburger BH (2008) Accumulation and clearance of alpha-synuclein aggregates demonstrated by time-lapse imaging. $\mathrm{J}$ Neurochem 106:529-540.

Papouli E, Chen S, Davies AA, Huttner D, Krejci L, Sung P, Ulrich HD (2005) Crosstalk between SUMO and ubiquitin on PCNA is mediated by recruitment of the helicase Srs2p. Mol Cell 19:123-133.

Park SS, Lee D (2006) Selective loss of dopaminergic neurons and formation of Lewy body-like aggregations in alpha-synuclein transgenic fly neuronal cultures. Eur $\mathrm{J}$ Neurosci 23:2908-2914.

Patrick GN, Zhou P, Kwon YT, Howley PM, Tsai LH (1998) p35, the neuronal-specific activator of cyclin-dependent kinase 5 (Cdk5) is degraded by the ubiquitinproteasome pathway. J Biol Chem 273:24057-24064.

Patrick GN, Zukerberg L, Nikolic M, de la Monte S, Dikkes P, Tsai LH (1999) Conversion of p35 to p25 deregulates Cdk5 activity and promotes neurodegeneration. Nature 402:615-622.

Peel AL, Klein RL (2000) Adeno-associated virus vectors: activity and applications in the CNS. J Neurosci Methods 98:95-104.

Pichler A, Gast A, Seeler JS, Dejean A, Melchior F (2002) The nucleoporin RanBP2 has SUMO1 E3 ligase activity. Cell 108:109-120.

Pichler A, Knipscheer P, Saitoh H, Sixma TK, Melchior F (2004) The RanBP2 SUMO E3 ligase is neither HECT- nor RING-type. Nat Struct Mol Biol 11:984-991.

Pichler A, Knipscheer P, Oberhofer E, van Dijk WJ, Korner R, Olsen JV, Jentsch S, Melchior F, Sixma TK (2005) SUMO modification of the ubiquitin-conjugating enzyme E2-25K. Nat Struct Mol Biol 12:264-269.

Polymeropoulos MH, Lavedan C, Leroy E, Ide SE, Dehejia A, Dutra A, Pike B, Root H, Rubenstein J, Boyer R, Stenroos ES, Chandrasekharappa S, Athanassiadou A, Papapetropoulos T, Johnson WG, Lazzarini AM, Duvoisin RC, Di lorio G, Golbe LI, Nussbaum RL (1997) Mutation in the alpha-synuclein gene identified in families with Parkinson's disease. Science 276:2045-2047.

Pommereit D, Wouters FS (2007) An NGF-induced Exo70-TC10 complex locally antagonises Cdc42-mediated activation of N-WASP to modulate neurite outgrowth. J Cell Sci 120:2694-2705.

Potts PR, Yu H (2005) Human MMS21/NSE2 is a SUMO ligase required for DNA repair. Mol Cell Biol 25:7021-7032.

Pountney DL, Huang Y, Burns RJ, Haan E, Thompson PD, Blumbergs PC, Gai WP (2003) SUMO-1 marks the nuclear inclusions in familial neuronal intranuclear inclusion disease. Exp Neurol 184:436-446.

Pronin AN, Morris AJ, Surguchov A, Benovic JL (2000) Synucleins are a novel class of substrates for $\mathrm{G}$ protein-coupled receptor kinases. J Biol Chem 275:26515-26522.

Rajan S, Plant LD, Rabin ML, Butler MH, Goldstein SA (2005) Sumoylation silences the plasma membrane leak $\mathrm{K}+$ channel K2P1. Cell 121:37-47.

Ren XR, Ming GL, Xie Y, Hong Y, Sun DM, Zhao ZQ, Feng Z, Wang Q, Shim S, Chen ZF, Song HJ, Mei L, Xiong WC (2004) Focal adhesion kinase in netrin-1 signaling. Nat Neurosci 7:1204-1212.

Reverter D, Lima CD (2005) Insights into E3 ligase activity revealed by a SUMO-RanGAP1Ubc9-Nup358 complex. Nature 435:687-692.

Riley BE, Zoghbi HY, Orr HT (2005) SUMOylation of the polyglutamine repeat protein, ataxin-1, is dependent on a functional nuclear localization signal. $\mathrm{J}$ Biol Chem 280:21942-21948.

Rodriguez MS, Dargemont C, Hay RT (2001) SUMO-1 conjugation in vivo requires both a consensus modification motif and nuclear targeting. J Biol Chem 276:12654-12659.

Ross S, Best JL, Zon LI, Gill G (2002) SUMO-1 modification represses Sp3 transcriptional activation and modulates its subnuclear localization. Mol Cell 10:831-842. 
Rott R, Szargel R, Haskin J, Shani V, Shainskaya A, Manov I, Liani E, Avraham E, Engelender S (2008) Monoubiquitylation of alpha-synuclein by seven in absentia homolog (SIAH) promotes its aggregation in dopaminergic cells. J Biol Chem 283:3316-3328.

Rui HL, Fan E, Zhou HM, Xu Z, Zhang Y, Lin SC (2002) SUMO-1 modification of the Cterminal KVEKVD of Axin is required for JNK activation but has no effect on Wnt signaling. J Biol Chem 277:42981-42986.

Sachdev S, Bruhn L, Sieber H, Pichler A, Melchior F, Grosschedl R (2001) PIASy, a nuclear matrix-associated SUMO E3 ligase, represses LEF1 activity by sequestration into nuclear bodies. Genes Dev 15:3088-3103.

Saitoh $\mathrm{H}$, Hinchey $\mathrm{J}(2000)$ Functional heterogeneity of small ubiquitin-related protein modifiers SUMO-1 versus SUMO-2/3. J Biol Chem 275:6252-6258.

Saitoh H, Uwada J, Azusa K (2009) Strategies for the expression of SUMO-modified target proteins in Escherichia coli. Methods Mol Biol 497:211-221.

Sambrook J, Maniatis T, Fritsch EF (1989) Molecular cloning : a laboratory manual, 2nd Edition. Cold Spring Harbor, N.Y.: Cold Spring Harbor Laboratory.

Sampson DA, Wang M, Matunis MJ (2001) The small ubiquitin-like modifier-1 (SUMO-1) consensus sequence mediates Ubc9 binding and is essential for SUMO-1 modification. J Biol Chem 276:21664-21669.

Sapetschnig A, Rischitor G, Braun H, Doll A, Schergaut M, Melchior F, Suske G (2002) Transcription factor Sp3 is silenced through SUMO modification by PIAS1. Embo $\mathrm{J}$ 21:5206-5215.

Schmidt D, Muller S (2002) Members of the PIAS family act as SUMO ligases for c-Jun and p53 and repress p53 activity. Proc Natl Acad Sci U S A 99:2872-2877.

Selkoe DJ (1999) Translating cell biology into therapeutic advances in Alzheimer's disease. Nature 399:A23-31.

Serpell LC, Berriman J, Jakes R, Goedert M, Crowther RA (2000) Fiber diffraction of synthetic alpha-synuclein filaments shows amyloid-like cross-beta conformation. Proc Natl Acad Sci U S A 97:4897-4902.

Shao R, Zhang FP, Rung E, Palvimo JJ, Huhtaniemi I, Billig H (2004) Inhibition of small ubiquitin-related modifier-1 expression by luteinizing hormone receptor stimulation is linked to induction of progesterone receptor during ovulation in mouse granulosa cells. Endocrinology 145:384-392.

Shevchenko A, Wilm M, Vorm O, Jensen ON, Podtelejnikov AV, Neubauer G, Shevchenko A, Mortensen P, Mann M (1996) A strategy for identifying gel-separated proteins in sequence databases by MS alone. Biochem Soc Trans 24:893-896.

Shimura H, Schlossmacher MG, Hattori N, Frosch MP, Trockenbacher A, Schneider R, Mizuno Y, Kosik KS, Selkoe DJ (2001) Ubiquitination of a new form of alphasynuclein by parkin from human brain: implications for Parkinson's disease. Science 293:263-269.

Shinbo Y, Niki T, Taira T, Ooe H, Takahashi-Niki K, Maita C, Seino C, Iguchi-Ariga SM, Ariga $H$ (2006) Proper SUMO-1 conjugation is essential to DJ-1 to exert its full activities. Cell Death Differ 13:96-108.

Singleton $A B$, Farrer $M$, Johnson J, Singleton $A$, Hague $S$, Kachergus J, Hulihan $M$, Peuralinna T, Dutra A, Nussbaum R, Lincoln S, Crawley A, Hanson M, Maraganore D, Adler C, Cookson MR, Muenter M, Baptista M, Miller D, Blancato J, Hardy J, Gwinn-Hardy K (2003) alpha-Synuclein locus triplication causes Parkinson's disease. Science 302:841.

Souza JM, Giasson BI, Chen Q, Lee VM, Ischiropoulos H (2000) Dityrosine cross-linking promotes formation of stable alpha -synuclein polymers. Implication of nitrative and oxidative stress in the pathogenesis of neurodegenerative synucleinopathies. J Biol Chem 275:18344-18349.

Spillantini MG, Schmidt ML, Lee VM, Trojanowski JQ, Jakes R, Goedert M (1997) Alphasynuclein in Lewy bodies. Nature 388:839-840.

Steffan JS, Agrawal N, Pallos J, Rockabrand E, Trotman LC, Slepko N, Illes K, Lukacsovich T, Zhu YZ, Cattaneo E, Pandolfi PP, Thompson LM, Marsh JL (2004) SUMO 
modification of Huntingtin and Huntington's disease pathology. Science 304:100104.

Stelter P, Ulrich HD (2003) Control of spontaneous and damage-induced mutagenesis by SUMO and ubiquitin conjugation. Nature 425:188-191.

Sternsdorf T, Jensen K, Reich B, Will H (1999) The nuclear dot protein sp100, characterization of domains necessary for dimerization, subcellular localization, and modification by small ubiquitin-like modifiers. J Biol Chem 274:12555-12566.

Takahashi-Fujigasaki J, Arai K, Funata N, Fujigasaki H (2006) SUMOylation substrates in neuronal intranuclear inclusion disease. Neuropathol Appl Neurobiol 32:92-100.

Takahashi Y, Kahyo T, Toh EA, Yasuda H, Kikuchi Y (2001) Yeast Ull1/Siz1 is a novel SUMO1/Smt3 ligase for septin components and functions as an adaptor between conjugating enzyme and substrates. J Biol Chem 276:48973-48977.

Tanaka K, Nishide J, Okazaki K, Kato H, Niwa O, Nakagawa T, Matsuda H, Kawamukai M, Murakami Y (1999) Characterization of a fission yeast SUMO-1 homologue, pmt3p, required for multiple nuclear events, including the control of telomere length and chromosome segregation. Mol Cell Biol 19:8660-8672.

Tang D, Chun AC, Zhang M, Wang JH (1997) Cyclin-dependent kinase 5 (Cdk5) activation domain of neuronal Cdk5 activator. Evidence of the existence of cyclin fold in neuronal Cdk5a activator. J Biol Chem 272:12318-12327.

Tatham MH, Chen Y, Hay RT (2003) Role of two residues proximal to the active site of Ubc9 in substrate recognition by the Ubc9.SUMO-1 thiolester complex. Biochemistry 42:3168-3179.

Tatham MH, Kim S, Jaffray E, Song J, Chen Y, Hay RT (2005) Unique binding interactions among Ubc9, SUMO and RanBP2 reveal a mechanism for SUMO paralog selection. Nat Struct Mol Biol 12:67-74.

Tatham MH, Jaffray E, Vaughan OA, Desterro JM, Botting CH, Naismith JH, Hay RT (2001) Polymeric chains of SUMO-2 and SUMO-3 are conjugated to protein substrates by SAE1/SAE2 and Ubc9. J Biol Chem 276:35368-35374.

Tempe D, Piechaczyk M, Bossis G (2008) SUMO under stress. Biochem Soc Trans 36:874878.

Tsai LH, Delalle I, Caviness VS, Jr., Chae T, Harlow E (1994) p35 is a neural-specific regulatory subunit of cyclin-dependent kinase 5. Nature 371:419-423.

Uchimura Y, Nakamura M, Sugasawa K, Nakao M, Saitoh H (2004) Overproduction of eukaryotic SUMO-1- and SUMO-2-conjugated proteins in Escherichia coli. Anal Biochem 331:204-206.

Ueda H, Goto J, Hashida H, Lin X, Oyanagi K, Kawano H, Zoghbi HY, Kanazawa I, Okazawa H (2002) Enhanced SUMOylation in polyglutamine diseases. Biochem Biophys Res Commun 293:307-313.

Um JW, Chung KC (2006) Functional modulation of parkin through physical interaction with SUMO-1. J Neurosci Res 84:1543-1554.

van Niekerk EA, Willis DE, Chang JH, Reumann K, Heise T, Twiss JL (2007) Sumoylation in axons triggers retrograde transport of the RNA-binding protein La. Proc Natl Acad Sci U S A 104:12913-12918.

Vertegaal AC, Andersen JS, Ogg SC, Hay RT, Mann M, Lamond Al (2006) Distinct and overlapping sets of SUMO-1 and SUMO-2 target proteins revealed by quantitative proteomics. Mol Cell Proteomics 5:2298-2310.

Vilar M, Chou HT, Luhrs T, Maji SK, Riek-Loher D, Verel R, Manning G, Stahlberg H, Riek R (2008) The fold of alpha-synuclein fibrils. Proc Natl Acad Sci U S A 105:86378642.

Volles MJ, Lansbury PT, Jr. (2003) Zeroing in on the pathogenic form of alpha-synuclein and its mechanism of neurotoxicity in Parkinson's disease. Biochemistry 42:78717878.

Werner A, Moutty MC, Moller U, Melchior F (2009) Performing in vitro sumoylation reactions using recombinant enzymes. Methods Mol Biol 497:187-199.

Wessel D, Flugge UI (1984) A method for the quantitative recovery of protein in dilute solution in the presence of detergents and lipids. Anal Biochem 138:141-143. 
Xu J, Kao SY, Lee FJ, Song W, Jin LW, Yankner BA (2002) Dopamine-dependent neurotoxicity of alpha-synuclein: a mechanism for selective neurodegeneration in Parkinson disease. Nat Med 8:600-606.

Yang SH, Galanis A, Witty J, Sharrocks AD (2006) An extended consensus motif enhances the specificity of substrate modification by SUMO. Embo J 25:5083-5093.

Yang W, Sheng H, Warner DS, Paschen W (2008) Transient global cerebral ischemia induces a massive increase in protein sumoylation. J Cereb Blood Flow Metab 28:269-279.

Zacharias DA, Violin JD, Newton AC, Tsien RY (2002) Partitioning of lipid-modified monomeric GFPs into membrane microdomains of live cells. Science 296:913-916.

Zarranz JJ, Alegre J, Gomez-Esteban JC, Lezcano E, Ros R, Ampuero I, Vidal L, Hoenicka J, Rodriguez O, Atares B, Llorens V, Gomez Tortosa E, del Ser T, Munoz DG, de Yebenes JG (2004) The new mutation, E46K, of alpha-synuclein causes Parkinson and Lewy body dementia. Ann Neurol 55:164-173.

Zhang FP, Mikkonen L, Toppari J, Palvimo JJ, Thesleff I, Janne OA (2008a) Sumo-1 function is dispensable in normal mouse development. Mol Cell Biol 28:5381-5390.

Zhang $\mathrm{H}$, Saitoh $\mathrm{H}$, Matunis MJ (2002) Enzymes of the SUMO modification pathway localize to filaments of the nuclear pore complex. Mol Cell Biol 22:6498-6508.

Zhang J, Cicero SA, Wang L, Romito-Digiacomo RR, Yang Y, Herrup K (2008b) Nuclear localization of Cdk5 is a key determinant in the postmitotic state of neurons. Proc Natl Acad Sci U S A 105:8772-8777.

Zhao X, Blobel G (2005) A SUMO ligase is part of a nuclear multiprotein complex that affects DNA repair and chromosomal organization. Proc Natl Acad Sci U S A 102:4777-4782.

Zhou C, Huang Y, Przedborski S (2008) Oxidative stress in Parkinson's disease: a mechanism of pathogenic and therapeutic significance. Ann N Y Acad Sci 1147:93104.

Zolotukhin S, Byrne BJ, Mason E, Zolotukhin I, Potter M, Chesnut K, Summerford C, Samulski RJ, Muzyczka N (1999) Recombinant adeno-associated virus purification using novel methods improves infectious titer and yield. Gene Ther 6:973-985. 


\section{Curriculum Vitae}

Personal details:

Name: Petranka Krumova

Nationality: Bulgarian

Date and place of birth: 14.10.1981 in Sofia, Bulgaria

Education:

October 2005 - March 2009

PhD candidate in the Neurobiological Research Laboratory, University Hospital Göttingen, Germany

October 2004 - September 2005

International Max Planck Research School for Molecular Biology, Göttingen, Germany

October 2000- June 2003

B.Sc. in Molecular Biology

Sofia University "St. Kliment Ohridsky"

Sofia, Bulgaria

Scholarships:

$2005-2008$

Neuroscience Early Stage Research Trainining (NEUREST)

Marie Curie Actions Fellowship

$2004-2005$

International Max Planck Research School

Stipend

Scientific courses:

13-15 December 2005

Scientific Communication,

Göttinger Zentrum für Molekular Biologie,

Göttingen, Germany

21-22 August 2006

Working with protein structures. Computerbased data analysis, University of Göttingen, Germany

5-12 September 2006

EMBO Practical Course on Ubiquitin and SUMO, Split, Croatia

9-11 July 2007

Confocal Microscopy, University of Göttingen, Germany 


\section{Conferences}

8-12 July 2006

16-21 January 2007

29 March -1 April 2007

1-5 September 2007

12-16 July 2008

15-19 November 2008 $5^{\text {th }}$ Forum of European Neuroscience, Vienna, Austria

Keystone Meeting - Molecular Mechanisms in Neurodegeneration, Taos, USA

German Neuroscience Society Meeting, Göttingen, Germany

European Life Science Organization Meeting, Dresden, Germany

$6^{\text {th }}$ Forum of European Neuroscience, Geneva, Switzerland

Poster contribution

Society for Neuroscience (SfN) 2008 Meeting Washington, USA

Poster contribution 


\section{Publications}

Krumova P., Meulmeester E., Garrido M., Tirard M., Hsiao H.H., Karpinar P., Ruonala M., Zweckstetter M., Wouters F.S., Urlaub H., Brose N., Kügler S., Melchior M., Bähr M., Weishaupt J.H. (2009). SUMOylation regulates a-synuclein toxicity. (In preparation)

Krumova P., Eckermann K., Bähr M., Weishaupt J.H. (2009). The regulator of CDK5 - p35 is a novel SUMO target. (In preparation) 